\title{
Self-Assembly of Metallo-Supramolecules with Dissymmetrical Ligands and Characterization by Scanning Tunneling Microscopy
}

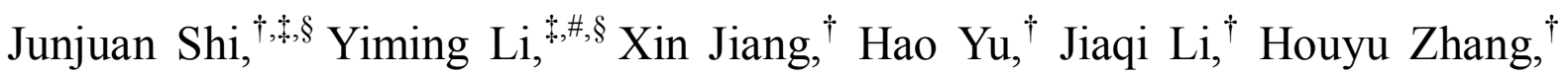

Daniel J. Trainer, ${ }^{\perp}$ Saw Wai Hla ${ }^{\perp}$ Heng Wang, ${ }^{\ddagger}$ Ming Wang ${ }^{\dagger, *}$ and Xiaopeng $\mathrm{Li}^{\ddagger \pm}, *$

†State Key Laboratory of Supramolecular Structure and Materials, College of Chemistry, Jilin University, Changchun, Jilin 130012, China

College of Chemistry and Environmental Engineering, Shenzhen University, Shenzhen, Guangdong 518055, China

\#Department of Chemistry, University of South Florida, Tampa, Florida 33620, United States ${ }^{\perp}$ Nanoscience and Technology Division, Argonne National Laboratory, Lemont, Illinois 60439, United States

${ }^{\perp}$ Shenzhen University General Hospital, Shenzhen University, Clinical Medical Academy, Shenzhen, Guangdong 518055, China 


\section{Table of Contents}

1. Experimental Section...................................................

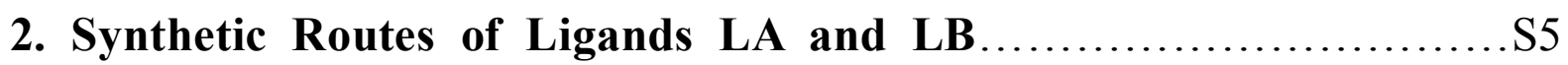

3. Synthesis of Ligands and Complexes............................. 7

4. ${ }^{1} \mathrm{H}$ NMR, ${ }^{13} \mathrm{C}$ NMR, 2D COSY NMR, 2D COSY NMR, DOSY, MALDI-TOF MS and ESI-MS Data............................... 32

5. UV-Vis Spectra of Ligands and Complexes........................ 110

6. DFT Calculations of the Binding Energies of A1-A4 _................S110

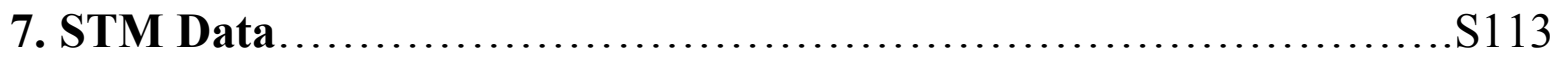

8. Reference..................................................... 120 


\section{Experimental Section}

All reagents were purchased from Sigma-Aldrich, Fisher, Acros, and Alfa Aesar. And they were used as received unless noted otherwise. Column chromatography was conducted using $\mathrm{SiO}_{2}$ (VWR, 40-60 $\mu \mathrm{m}, 60 \AA$ ) and the separated products were visualized by UV light.

Nuclear magnetic resonance (NMR). NMR spectra data were recorded at $25{ }^{\circ} \mathrm{C}$ on Bruker $600 \mathrm{MHz}$ and $400 \mathrm{MHz}$ nuclear magnetic resonance instruments using $\mathrm{CDCl}_{3}$ and $\mathrm{CD}_{3} \mathrm{CN}$ as the solvents.

Electrospray ionization-mass spectrometry (ESI-MS) and travelling wave ion mobility-mass spectrometry (TWIM-MS). Both spectra were collected on a Waters Synapt G2 mass spectrometer, using solutions of $0.01 \mathrm{mg}$ sample in $1 \mathrm{~mL}$ of $\mathrm{CHCl}_{3} / \mathrm{CH}_{3} \mathrm{OH}(1: 3$, $\mathrm{v} / \mathrm{v}$ ) or acetonitrile for the intermediate compounds, ligands and complexes. The TWIM-MS experiments were performed under the following conditions: ESI capillary voltage, $1-3 \mathrm{kV}$; sample cone voltage, $20-30 \mathrm{~V}$; extraction cone voltage, $3 \mathrm{~V}$; source temperature $100{ }^{\circ} \mathrm{C}$; desolvation temperature, $200{ }^{\circ} \mathrm{C}$; cone gas flow, $10 \mathrm{~L} / \mathrm{h}$; desolvation gas flow, $700 \mathrm{~L} / \mathrm{h}\left(\mathrm{N}_{2}\right)$; source gas control, $0 \mathrm{~mL} / \mathrm{min}$; trap gas control, $2 \mathrm{~mL} / \mathrm{min}$; helium cell gas control, 100 $\mathrm{mL} / \mathrm{min}$; ion mobility (IM) cell gas control, $30 \mathrm{~mL} / \mathrm{min}$; sample flow rate, $5 \mu \mathrm{L} / \mathrm{min}$; IM traveling wave height, $25 \mathrm{~V}$; and IM traveling wave velocity, $1000 \mathrm{~m} / \mathrm{s}$.

Matrix-assisted laser desorption/ionization time-of-flight (MALDI-TOF) mass spectrometry. MALDI-TOF characterization was conducted on a Bruker UltrafleXtreme TOF/TOF mass spectrometer (Bruker Daltonics, Inc., Billerica, MA) equipped with a Nd: YAG laser (355 nm). Trans-2-[3-(4-tert-butylphenyl)-2-methyl-2-propenylidene] malononitrile (DCTB, Aldrich, $>99 \%$ ) was served as matrix. The matrix dissolved in $\mathrm{CHCl}_{3}$ at $20 \mathrm{mg} \mathrm{mL}^{-1}$ and organic compounds were dissolved in $\mathrm{CHCl}_{3}$ at $10 \mathrm{mg} \mathrm{mL}^{-1}$. Sample was prepared by depositing $0.5 \mu \mathrm{L}$ of matrix on the wells of a 384-well ground-steel plate, allowing the spots to dry, depositing $0.5 \mu \mathrm{L}$ of the sample on a spot of dry matrix, and adding another $0.5 \mu \mathrm{L}$ of matrix on top of the dry sample. The plate was inserted into the MALDI source after drying. The sample was conducted in linear mode. And the data analysis was conducted with Bruker's FlexAnalysis software.

Ultraviolet visible (UV-Vis). All UV-Vis spectra were recorded on a HORIBA Scientific 
FluoroMax-4 spectrofluorometer at room temperature.

Scanning tunneling microscope (STM). UHV-LT-STM experiments were performed at $4 \mathrm{~K}$ using a home-built system with a Besoke-Beetle type STM scanner. The $\operatorname{Ag}(111)$ substrate was cleaned by repeated cycles of sputtering and annealing up to $1000 \mathrm{~K}$. An electrochemically etched polycrystalline tungsten wire was used for the STM tip. The tip apex was prepared by using a controlled tip-crash procedure. The samples were deposited onto the cleaned $\operatorname{Ag}(111)$ surface at $25^{\circ} \mathrm{C}$, and then cooled to $4 \mathrm{~K}$ inside the STM system.

TEM. The sample solutions $(0.5 \mathrm{mg} / \mathrm{mL}$ in DMF) were drop cast on copper grids (ultrathin carbon supported by a lacey carbon film on a 400 Mesh copper grid purchased from www.tedpella.com) and the extra solution was absorbed by filter paper to avoid aggregation. The grid was then washed with three drops of diethyl ether to remove DMF. The nanostructures were formed by diffusing diethyl ether into the sample solution $(4.0 \mathrm{mg} / \mathrm{mL}$ in DMF) slowly and the mixture were drop cast on copper grids directly. The TEM images were all taken with a FEI Morgagni transmission electron microscope.

Molecular modeling. Energy minimization was conducted with Materials Studio version 4.2, using the Anneal and Geometry Optimization tasks in the Forcite module (Accelrys Software, Inc.). The counterions were omitted in the modeling. Geometry optimization was conducted using a universal force field with atom-based summation and cubic spline truncation for both the electrostatic and van der Waals parameters.

DFT calculations. The calculations are performed at DFT level with PBE0 functional. The basis sets including effective core potential of LANL1MB for $\mathrm{Zn}$ atoms and 3-21G for the ligands are used for the sake of saving the computational cost. The supermolecular approach is used to calculate the interaction energy of the dimer complexes as the energy difference between the energy of the complex and the energies of the isolated ligands. The basis set superposition error (BSSE) is ignored because of DFT method and relatively small basis set. 


\section{Synthetic Routes of Ligands LA and LB}

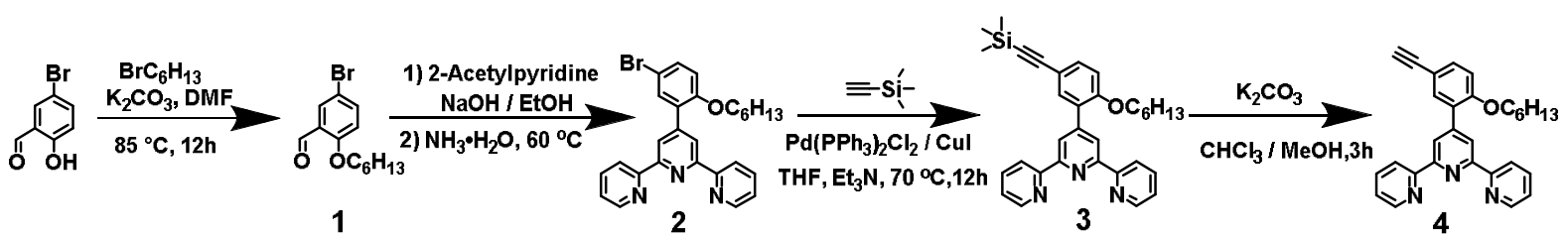

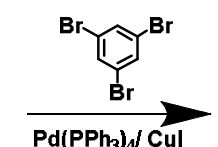

$\mathrm{Pd}\left(\mathrm{PPh}_{3}\right)_{4} \mathrm{Cul}$ THF, Et $\mathrm{t}_{3} \mathrm{~N}, 70^{\circ} \mathrm{C}, 12 \mathrm{~h}$

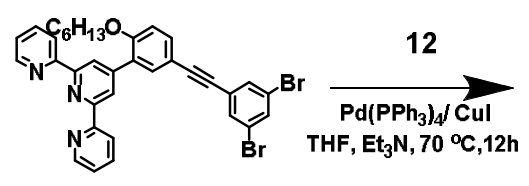

5

1) 2-Acetylpyridine

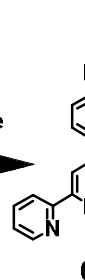

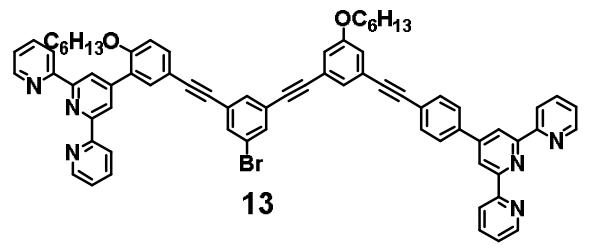

Br<smiles>O=C(O)C=C=[AlH2]</smiles>

$\mathrm{Pd}\left(\mathrm{PPh}_{3} \mathrm{C}_{2} \mathrm{Cl}_{2} / \mathrm{Cul}\right.$

6

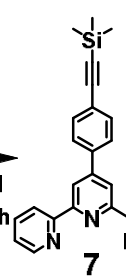

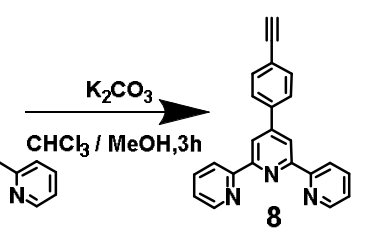

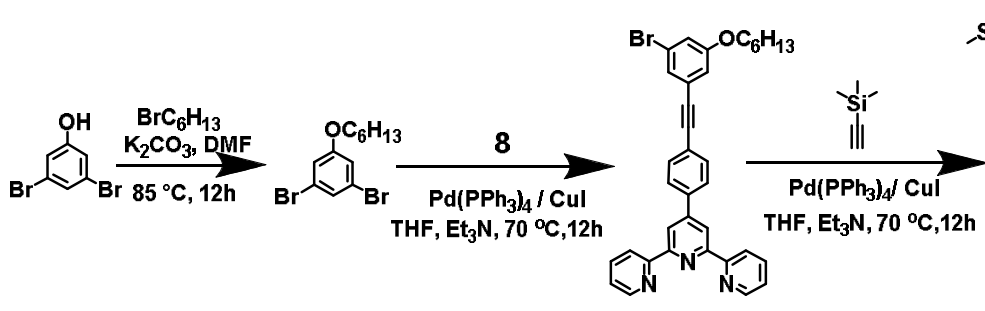

9
10

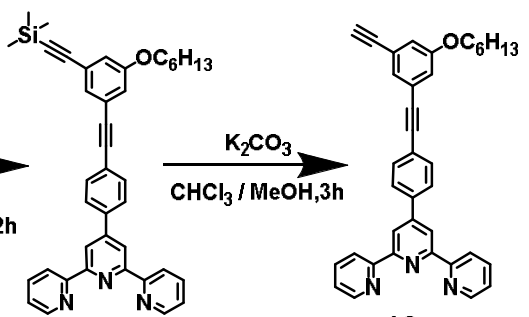

11

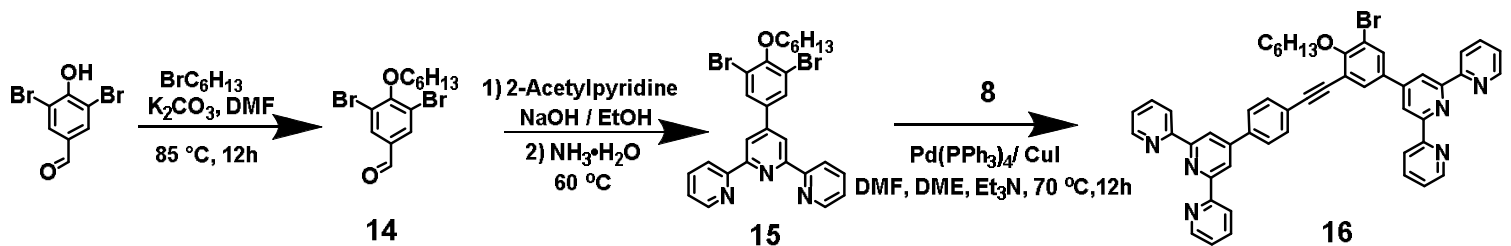

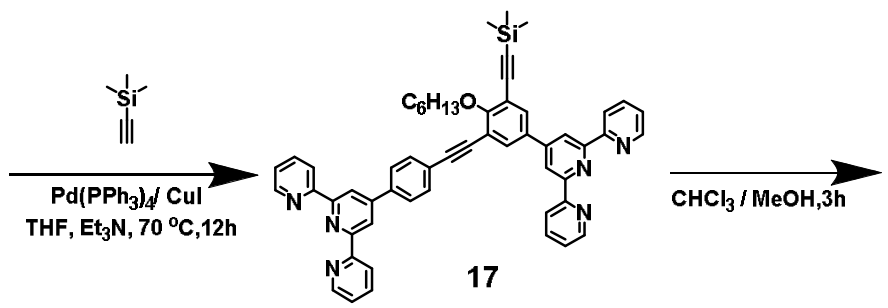

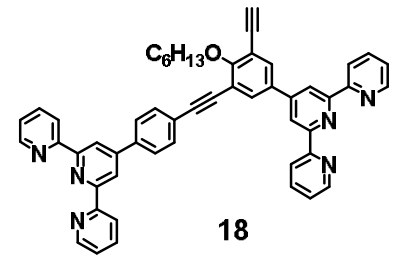

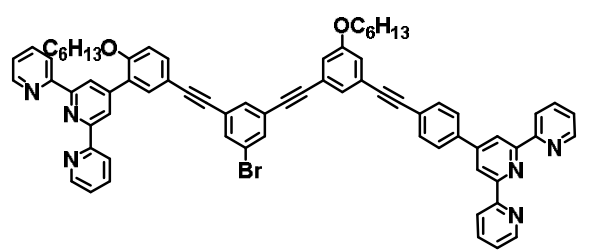

13

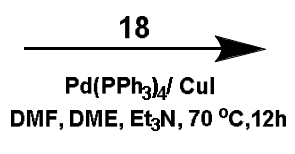
DMF, DME, Et $\mathrm{t}_{3} \mathrm{~N}, 70^{\circ} \mathrm{C}, 12 \mathrm{~h}$

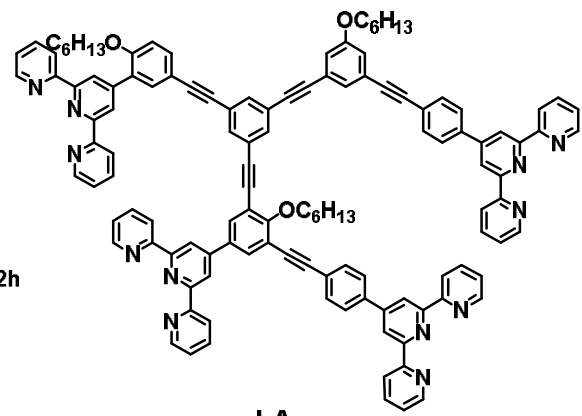

LA

Scheme S1. Synthesis of ligand LA. 

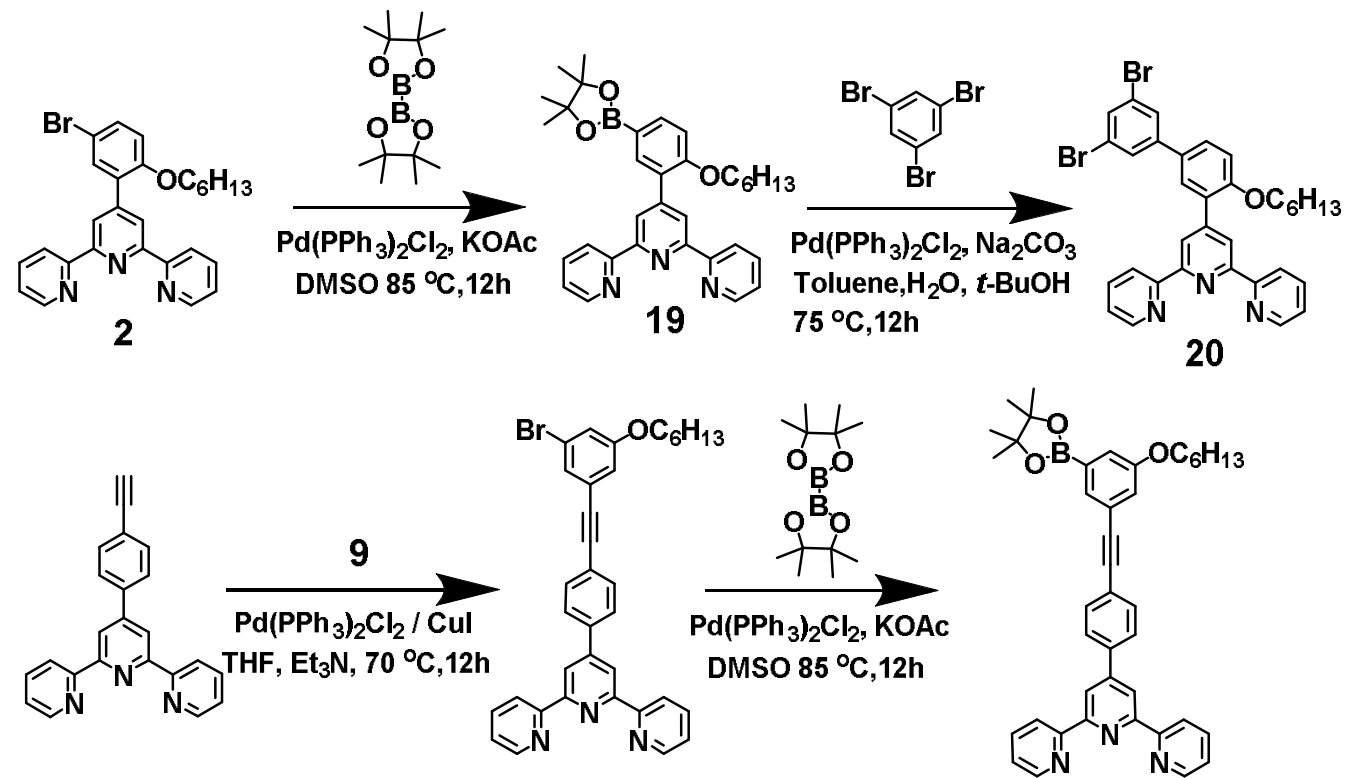

8

10

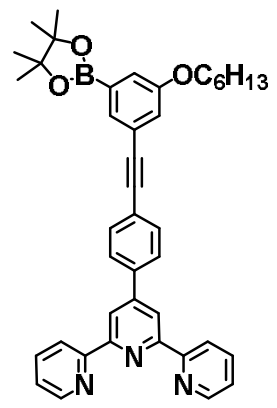

21

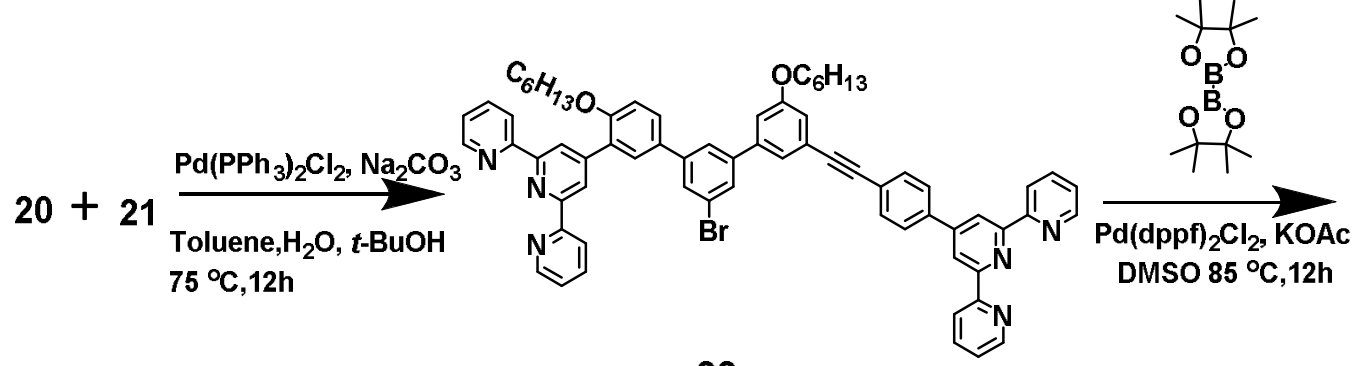

22

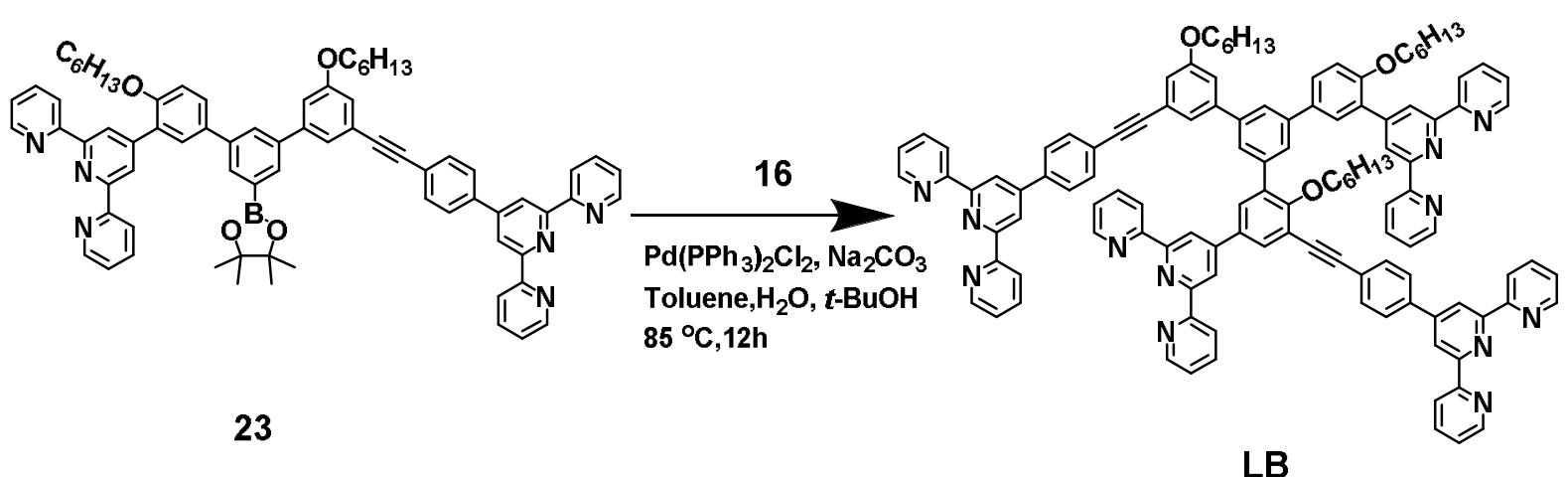

Scheme S2. Synthesis of ligand LB. 


\section{Synthesis of Ligands and Complexes}

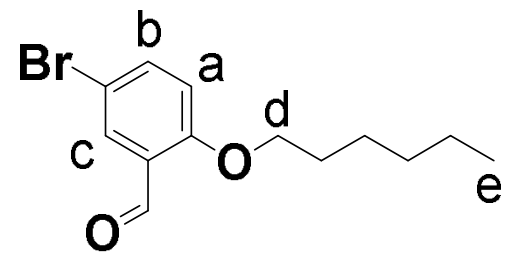

Compound 1. Compound 1 was synthesized according to literature. ${ }^{1}$ To a solution of 2-hydroxyl-5-bromo benzaldehyde (10 g, $50 \mathrm{mmol})$ in DMF $(45 \mathrm{~mL}), 1$-bromohexane $(9 \mathrm{~g}$, $60 \mathrm{mmol})$, and $\mathrm{K}_{2} \mathrm{CO}_{3}(14 \mathrm{~g}, 100 \mathrm{mmol})$ were added and the mixture was heated at $85{ }^{\circ} \mathrm{C}$ overnight. The reaction mixture was cooled to room temperature, extracted with $\mathrm{CH}_{2} \mathrm{Cl}_{2}$ and further purified by silica gel column chromatography with $\mathrm{CH}_{2} \mathrm{Cl}_{2}$ : hexane (1:4) to afford the product as light yellow oil $(8.5 \mathrm{~g}, 91 \%) .{ }^{1} \mathrm{H} \mathrm{NMR}\left(600 \mathrm{MHz}, \mathrm{CDCl}_{3}\right) \delta 10.42(\mathrm{~s}, 1 \mathrm{H}$, CHO-H), $7.92\left(\mathrm{~d}, J=2.6 \mathrm{~Hz}, 1 \mathrm{H}, \mathrm{Ph}-H^{\mathrm{c}}\right), 7.60\left(\mathrm{dd}, J=8.9,2.7 \mathrm{~Hz}, 1 \mathrm{H}, \mathrm{Ph}-H^{\mathrm{a}}\right), 6.88(\mathrm{~d}, J=$ $\left.8.8 \mathrm{~Hz}, 1 \mathrm{H}, \mathrm{Ph}-H^{\mathrm{b}}\right), 4.06\left(\mathrm{t}, J=6.4 \mathrm{~Hz}, 2 \mathrm{H}, \mathrm{Alkyl}-H^{\mathrm{d}}\right), 1.84$ ( $2 \mathrm{H}$, Alkyl- $\left.H\right), 1.50-1.44$ (m, 2H, Alkyl- $H$ ), 1.35 (4H, Alkyl- $H), 0.93-0.89$ (m, 3H, Alkyl- $\left.H^{\mathrm{e}}\right) .{ }^{13} \mathrm{C}$ NMR (150 MHz, $\left.\mathrm{CDCl}_{3}\right) \delta 188.2,160.3,138.1,130.5,125.9,114.5,113.0,68.9,31.4,28.9,25.6,22.5,14.0$.

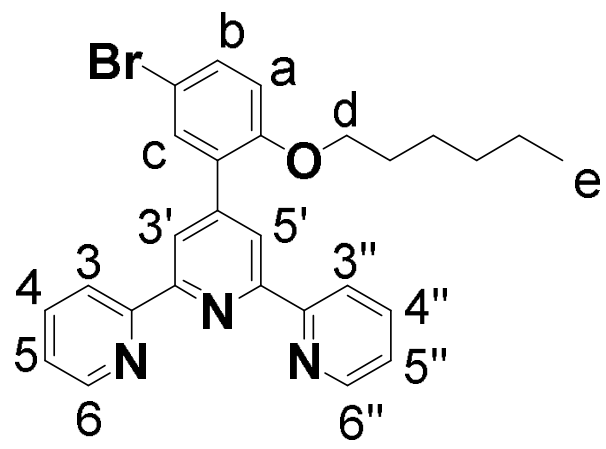

Compound 2. Compound 2 was synthesized according to literature. ${ }^{1}$ To a solution of $\mathrm{NaOH}$ powder $(3.2 \mathrm{~g}, 80 \mathrm{mmol})$ in $70 \mathrm{~mL}$ of $\mathrm{EtOH}$, compound 1 (4.3 g, $15 \mathrm{mmol})$ and 2-acetylpyridine $(4.0 \mathrm{~g}, 33 \mathrm{mmol})$ were added. After stirring at $25^{\circ} \mathrm{C}$ for $10 \mathrm{~h}$, aqueous $\mathrm{NH}_{3} \cdot \mathrm{H}_{2} \mathrm{O}(50 \mathrm{~mL})$ was added and the mixture was refluxed for $20 \mathrm{~h}$. After cooling to room temperature, ethanol was removed under reduced pressure. The aqueous phase was extracted using $\mathrm{CH}_{2} \mathrm{Cl}_{2}$ and the organic layer was washed with water for three times. After the solvent was removed under reduced pressure, the residue was purified by silica gel chromatography 
with $\mathrm{CH}_{2} \mathrm{Cl}_{2}$ as eluent to afford compound 2 as white solid $(5.5 \mathrm{~g}, 75 \%) .{ }^{1} \mathrm{H} \mathrm{NMR}(600 \mathrm{MHz}$, $\left.\mathrm{CDCl}_{3}\right) \delta 8.70\left(\mathrm{ddd}, J=5.1,1.8,0.8 \mathrm{~Hz}, 2 \mathrm{H}, \operatorname{tpy}-H^{6,6 "}\right), 8.67(\mathrm{dd}, J=8.0,1.2 \mathrm{~Hz}, 2 \mathrm{H}$, tpy- $\left.H^{3,3 "}\right), 8.65\left(\mathrm{~s}, 2 \mathrm{H}, \mathrm{tpy}-H^{3^{\prime}, 5^{\prime}}\right), 7.87\left(\mathrm{td}, J=7.7,1.8 \mathrm{~Hz}, 2 \mathrm{H}, \mathrm{tpy}-H^{4,4 "}\right), 7.68(\mathrm{~d}, J=2.5 \mathrm{~Hz}$, $\left.1 \mathrm{H}, \mathrm{Ph}-H^{\mathrm{b}}\right), 7.45\left(\mathrm{dd}, J=8.8,2.5 \mathrm{~Hz}, 1 \mathrm{H}, \mathrm{Ph}-H^{\mathrm{a}}\right), 7.34(\mathrm{ddd}, J=7.5,4.7,1.2 \mathrm{~Hz}, 2 \mathrm{H}$, tpy- $\left.H^{5,5^{\prime \prime}}\right), 6.88\left(\mathrm{~d}, J=8.8 \mathrm{~Hz}, 1 \mathrm{H}, \mathrm{Ph}-H^{\mathrm{c}}\right), 3.98\left(\mathrm{t}, J=6.3 \mathrm{~Hz}, 2 \mathrm{H}\right.$, Alkyl- $\left.H^{\mathrm{d}}\right), 1.74-1.66(\mathrm{~m}$, 2H, Alkyl- $H$ ), $1.42-1.33$ (m, 2H, Alkyl- $H$ ), $1.20-1.06$ (m, 4H, Alkyl- $H$ ), 0.72 (t, $J=7.2$ $\mathrm{Hz}, 3 \mathrm{H}$, Alkyl- $\left.H^{\mathrm{e}}\right) .{ }^{13} \mathrm{C} \mathrm{NMR}\left(150 \mathrm{MHz}, \mathrm{CDCl}_{3}\right) \delta 156.3,155.5,155.2,149.1,147.0,136.7$, $132.9,132.5,130.3,123.7,121.6,121.2,114.0,112.9,68.8,31.5,29.1,25.8,22.4,14.0$. ESI-MS (m/z): Calcd. for $\left[\mathrm{C}_{27} \mathrm{H}_{27} \mathrm{BrN}_{3} \mathrm{O}+\mathrm{H}\right]^{+}:$488.13, Found : 488.08 .

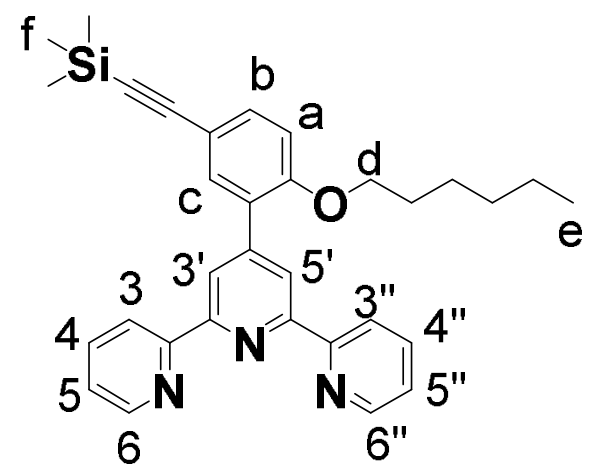

Compound 3. Compound 3 was synthesized using the reported method. ${ }^{2}$ A mixture of compound 2 (3.5 g, $7.2 \mathrm{mmol}), \mathrm{Pd}\left(\mathrm{PPh}_{3}\right)_{4}(323 \mathrm{mg}, 0.28 \mathrm{mmol})$ and copper(I) iodide (25 mg, $0.14 \mathrm{mmol}$ ) was degassed under nitrogen for three times. After that, $40 \mathrm{~mL}$ anhydrous THF, $20 \mathrm{~mL}$ anhydrous $\mathrm{Et}_{3} \mathrm{~N}$, and (trimethylsilyl)acetylene $(2.5 \mathrm{~mL}, 18 \mathrm{mmol})$ were added subsequently. The mixture was stirred under $70{ }^{\circ} \mathrm{C}$ overnight. After cooling down to room temperature, solvent was removed under vacuum, and the residue was extracted with $\mathrm{CH}_{2} \mathrm{Cl}_{2}$. The combined organic phase was washed with brine and dried over $\mathrm{Na}_{2} \mathrm{SO}_{4}$. After removal of solvent under vacuum, the residue was purified by silica gel chromatography with $\mathrm{CH}_{2} \mathrm{Cl}_{2}$ : methanol (100:0.5) as eluent to afford a yellow solid (3.0 g, 83\%). ${ }^{1} \mathrm{H}$ NMR (600 MHz, $\left.\mathrm{CDCl}_{3}\right) \delta 8.71\left(\mathrm{~d}, J=6.0 \mathrm{~Hz}, 2 \mathrm{H}, \mathrm{tpy}-H^{6,6^{\prime \prime}}\right), 8.68-8.67\left(\mathrm{~m}, 6 \mathrm{H}, \mathrm{tpy}-H^{3,3^{\prime \prime}}\right.$ and tpy- $\left.H^{3^{\prime}, 5^{\prime}}\right), 7.86$ $\left(\mathrm{t}, J=7.5 \mathrm{~Hz}, 2 \mathrm{H}, \mathrm{tpy}-H^{4,4 "}\right), 7.71\left(\mathrm{~d}, J=2.1 \mathrm{~Hz}, 1 \mathrm{H}, \mathrm{Ph}-H^{\mathrm{c}}\right), 7.48(\mathrm{dd}, J=8.8,2.1 \mathrm{~Hz}, 1 \mathrm{H}$, $\left.\mathrm{Ph}-H^{\mathrm{b}}\right), 7.34\left(\mathrm{dd}, J=7.2,4.8 \mathrm{~Hz}, 2 \mathrm{H}, \mathrm{tpy}-H^{5,5 "}\right), 6.91\left(\mathrm{~d}, J=8.5 \mathrm{~Hz}, 1 \mathrm{H}, \mathrm{Ph}-H^{\mathrm{a}}\right), 4.02(\mathrm{t}, J=$ $6.2 \mathrm{~Hz}, 2 \mathrm{H}$, Alkyl- $\left.H^{\mathrm{d}}\right), 1.70$ (2H, Alkyl- $\left.H\right), 1.44-1.29$ (m, 2H, Alkyl- $H$ ), 1.21-1.03 (m, 4H, Alkyl- $H), 0.72\left(\mathrm{t}, J=7.0 \mathrm{~Hz}, 3 \mathrm{H}\right.$, Alkyl- $\left.H^{\mathrm{e}}\right) .0 .26\left(\mathrm{~s}, 9 \mathrm{H}, H^{\mathrm{f}}\right) .{ }^{13} \mathrm{C} \mathrm{NMR}\left(150 \mathrm{MHz}, \mathrm{CDCl}_{3}\right) \delta$ 
$156.7,156.6,155.3,149.2,147.7,136.9,134.4,133.9,123.7,128.5,121.9,121.4,115.5$, 112.1, 105.0, 93.1, 68.7, 31.6, 29.2, 25.9, 22.5, 14.0, 0.2. ESI-MS $(\mathrm{m} / \mathrm{z})$ : Calcd. for $\left[\mathrm{C}_{27} \mathrm{H}_{27} \mathrm{BrN}_{3} \mathrm{O}+\mathrm{H}\right]^{+}:$506.25, Found: 506.21.

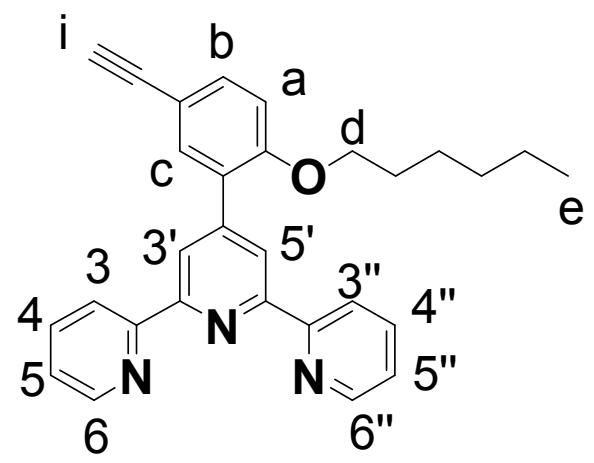

Compound 4. Compound 4 was synthesized using the reported method. ${ }^{2}$ To a flask containing solution of compound $\mathbf{3}(2.4 \mathrm{~g}, 4.8 \mathrm{mmol})$ in $\mathrm{CHCl}_{3}(50 \mathrm{~mL})$ and $\mathrm{MeOH}(50 \mathrm{~mL})$, $\mathrm{K}_{2} \mathrm{CO}_{3}(2.1 \mathrm{~g}, 15 \mathrm{mmol})$ was added. After being stirred at room temperature for $4 \mathrm{~h}$, the mixture was extracted with $\mathrm{CHCl}_{3}$ and further purified by silica gel chromatography with chloroform: methanol (100:1) as eluent to afford the product as a light-yellow solid (1.6 g, 89\%). ${ }^{1} \mathrm{H}$ NMR (600 MHz, $\left.\mathrm{CDCl}_{3}\right) \delta 8.71-8.69\left(\mathrm{~d}, J=4.8 \mathrm{~Hz}, 2 \mathrm{H}\right.$, tpy- $\left.H^{6,6 "}\right), 8.67(\mathrm{~m}, 4 \mathrm{H}$, tpy- $H^{3,5^{\prime}}$ and tpy- $\left.H^{3,3 "}\right), 7.87\left(\mathrm{td}, J=7.7,1.7 \mathrm{~Hz}, 2 \mathrm{H}, \operatorname{tpy}-H^{4,4 "}\right), 7.72(\mathrm{~d}, J=2.1 \mathrm{~Hz}, 1 \mathrm{H}$, Ph- $H^{\mathrm{c}}$ ), 7.50 (dd, $\left.J=8.5,2.1 \mathrm{~Hz}, 1 \mathrm{H}, \mathrm{Ph}-H^{b}\right), 7.33$ (dd, $J=7.4,4.8 \mathrm{~Hz}, 2 \mathrm{H}$, tpy- $\left.H^{5,5 "}\right), 6.94$ (d, $\left.J=8.5 \mathrm{~Hz}, 1 \mathrm{H}, \mathrm{Ph}-H^{\mathrm{a}}\right), 4.02\left(\mathrm{t}, J=6.2 \mathrm{~Hz}, 2 \mathrm{H}\right.$, Alkyl $\left.-H^{\mathrm{d}}\right), 3.04\left(\mathrm{~s}, 1 \mathrm{H}, H^{\mathrm{i}}\right), 1.74-1.70(\mathrm{~m}$, 2H, Alkyl- $H$ ), 1.39 (t, $J=7.7 \mathrm{~Hz}, 2 \mathrm{H}$, Alkyl- $H$ ), $1.18-1.09$ (m, 4H, Alkyl- $H$ ), 0.71 (d, $J=$ $7.0 \mathrm{~Hz}, 3 \mathrm{H}$, Alkyl- $\left.H^{\mathrm{e}}\right) .{ }^{13} \mathrm{C} \mathrm{NMR}\left(150 \mathrm{MHz}, \mathrm{CDCl}_{3}\right) \delta 156.8,156.5,155.3,149.2,147.5$, 136.8, 134.4, 134.0, 128.6, 123.7, 121.8, 121.3, 114.4, 112.1, 83.5, 76.4, 68.7, 31.6, 29.1, 25.8, 22.4, 14.0. ESI-MS (m/z): Calcd. for $\left[\mathrm{C}_{29} \mathrm{H}_{27} \mathrm{~N}_{3} \mathrm{O}+\mathrm{H}\right]^{+}: 434.22$. Found 434.18. 


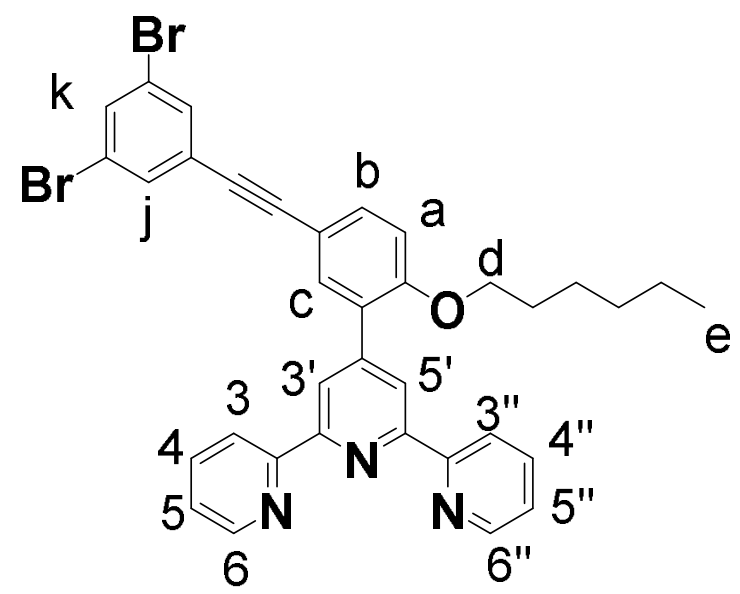

Compound 5. A mixture of compound 4 (2.6 g, $6 \mathrm{mmol})$, 1,3,5-tribromobenzene (5.6 g, 18 mmol), $\mathrm{Pd}\left(\mathrm{PPh}_{3}\right)_{4}(346 \mathrm{mg}, 0.3 \mathrm{mmol})$ and copper(I) iodide $(34 \mathrm{mg}, 0.18 \mathrm{mmol}$ ) was degassed under nitrogen for three times. After that, $40 \mathrm{~mL}$ anhydrous THF, $20 \mathrm{~mL}$ anhydrous $\mathrm{Et}_{3} \mathrm{~N}$ were added subsequently. The mixture was stirred under $70{ }^{\circ} \mathrm{C}$ overnight. After cooling down to room temperature, solvent was removed under vacuum, and the residue was extracted with $\mathrm{CH}_{2} \mathrm{Cl}_{2}$. The combined organic phase was washed with brine and dried over $\mathrm{Na}_{2} \mathrm{SO}_{4}$. After removal of solvent under vacuum, the residue was purified by silica gel chromatography with $\mathrm{CH}_{2} \mathrm{Cl}_{2}$ : methanol (100:0.5) as eluent to afford a yellow solid $(7.7 \mathrm{~g}$, 67\%). ${ }^{1} \mathrm{H}$ NMR (600 MHz, $\left.\mathrm{CDCl}_{3}\right) \delta 8.71\left(\mathrm{ddd}, J=4.8,1.8,0.9 \mathrm{~Hz}, 2 \mathrm{H}, \mathrm{tpy}-H^{6,6 "}\right), 8.70$ (s, $\left.2 \mathrm{H}, \operatorname{tpy}-H^{3^{\prime} 5^{\prime}}\right), 8.68\left(\mathrm{dt}, J=8.0,1.1 \mathrm{~Hz}, 2 \mathrm{H}, \mathrm{tpy}-H^{3,3 "}\right), 7.88\left(\mathrm{td}, J=7.7,1.8 \mathrm{~Hz}, 2 \mathrm{H}, \operatorname{tpy}-H^{4,4 "}\right)$, $7.76\left(\mathrm{~d}, J=2.1 \mathrm{~Hz}, 1 \mathrm{H}, \mathrm{Ph}-H^{\mathrm{c}}\right), 7.61\left(\mathrm{~s}, 3 \mathrm{H}, \mathrm{Ph}-H^{\mathrm{j}}\right.$ and $\left.\mathrm{Ph}-H^{\mathrm{k}}\right), 7.52(\mathrm{dd}, J=8.5,2.1 \mathrm{~Hz}, 1 \mathrm{H}$, $\left.\mathrm{Ph}-H^{\mathrm{b}}\right), 7.34\left(\mathrm{ddd}, J=7.5,4.7,1.2 \mathrm{~Hz}, 2 \mathrm{H}, \mathrm{tpy}-H^{5,5^{\prime \prime}}\right), 6.98\left(\mathrm{~d}, J=8.6 \mathrm{~Hz}, 1 \mathrm{H}, \mathrm{Ph}-H^{\mathrm{a}}\right), 4.05(\mathrm{t}$, $J=6.2 \mathrm{~Hz}, 2 \mathrm{H}$, Alkyl- $\left.H^{\mathrm{d}}\right), 1.73$ (m, 2H, Alkyl-H), 1.41 (m, 2H, Alkyl- $\left.H\right), 1.22-1.07$ (m, 4H, Alkyl- $H), 0.73\left(\mathrm{t}, J=7.2 \mathrm{~Hz}, 3 \mathrm{H}\right.$, Alkyl- $\left.H^{\mathrm{e}}\right) .{ }^{13} \mathrm{C} \mathrm{NMR}\left(150 \mathrm{MHz}, \mathrm{CDCl}_{3}\right) \delta 157.1,156.5$, $155.3,149.2,147.4,136.9,134.2,133.6,133.6,132.9,128.8,127.1,123.7,122.7,121.8$, 121.3, 114.5, 112.3, 92.1, 85.8, 68.8, 31.6, 29.2, 25.9, 22.5, 14.0. ESI-MS (m/z): Calcd. for $\left[\mathrm{C}_{35} \mathrm{H}_{29} \mathrm{Br}_{2} \mathrm{~N}_{3} \mathrm{O}+\mathrm{H}\right]^{+}:$666.07. Found: 666.02. 


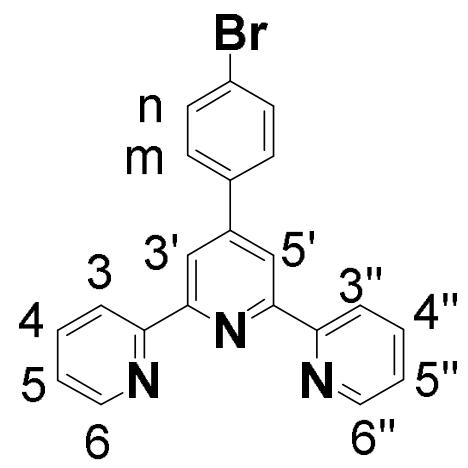

Compound 6. Compound 6 was synthesized using the reported method. ${ }^{2-3}$ To a solution of $\mathrm{NaOH}$ powder $(7.2 \mathrm{~g}, 180 \mathrm{mmol})$ in $\mathrm{EtOH}(200 \mathrm{~mL})$, 4-Bromobenzaldehyde $(5.55 \mathrm{~g}, 30$ mmol), and 2-acetylpyridine $(8.00 \mathrm{~g}, 66 \mathrm{mmol})$ were added. After stirring at room temperature for $24 \mathrm{~h}$, aqueous $\mathrm{NH}_{3} \cdot \mathrm{H}_{2} \mathrm{O}(25-28 \%, 120 \mathrm{~mL})$ was added, the resulting mixture was refluxed for $24 \mathrm{~h}$. After cooling to room temperature, the solid was collected by suction filtration and was washed with cold ethanol to give the product as a white solid $(8.5 \mathrm{~g}, 73.0 \%)$ was obtained. ${ }^{1} \mathrm{H} \mathrm{NMR}\left(600 \mathrm{MHz}, \mathrm{CDCl}_{3}\right) \delta 8.75-8.72$ (ddd, $J=4.8,1.8,0.9 \mathrm{~Hz}, 2 \mathrm{H}$, tpy- $\left.H^{6,6^{\prime \prime}}\right), 8.71\left(\mathrm{~s}, 2 \mathrm{H}, \mathrm{tpy}-H^{3^{\prime}, 5^{\prime}}\right), 8.68\left(\mathrm{dd}, J=8.0,1.1 \mathrm{~Hz}, 2 \mathrm{H}, \operatorname{tpy}-H^{3,3 "}\right), 7.89(\mathrm{td}, J=7.7$, $\left.1.8 \mathrm{~Hz}, 2 \mathrm{H}, \mathrm{tpy}-H^{4,4 "}\right), 7.80-7.77\left(\mathrm{~m}, 2 \mathrm{H}, \mathrm{Ph}-H^{\mathrm{m}}\right), 7.67-7.63\left(\mathrm{~m}, 2 \mathrm{H}, \mathrm{Ph}-H^{\mathrm{n}}\right), 7.37(\mathrm{ddd}, J$ $\left.=7.4,4.7,1.2 \mathrm{~Hz}, 2 \mathrm{H}, \operatorname{tpy}-H^{5,5 "}\right) .{ }^{13} \mathrm{C} \mathrm{NMR}\left(150 \mathrm{MHz}, \mathrm{CDCl}_{3}\right) \delta 156.2,156.2,149.3,149.1$, 137.5, 137.0, 132.2, 129.0, 124.1, 123.6, 121.5, 118.7. ESI-MS $(\mathrm{m} / \mathrm{z})$ : Calcd. for $\left[\mathrm{C}_{21} \mathrm{H}_{14} \mathrm{BrN}_{3}+\mathrm{H}\right]^{+}:$388.04. Found: 388.00 .

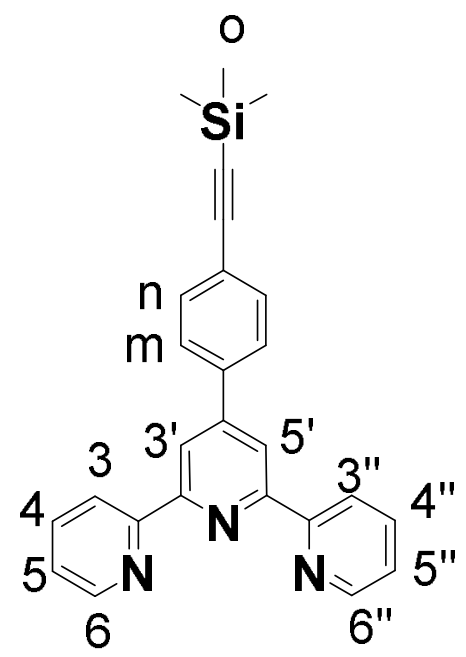

Compound 7. Compound 7 was synthesized using the reported method. ${ }^{2-3}$ Compound 6 (6.0 g, $15.5 \mathrm{mmol}), \mathrm{Pd}\left(\mathrm{PPh}_{3}\right)_{2} \mathrm{Cl}_{2}(542.5 \mathrm{mg}, 0.775 \mathrm{mmol})$ and $\mathrm{CuI}(88.5 \mathrm{mg}, 0.46 \mathrm{mmol})$ were mixed in a $200 \mathrm{~mL}$ Schlenk flask. After degassing and backfill with nitrogen for three times, 
$50 \mathrm{~mL}$ THF, and $30 \mathrm{~mL} \mathrm{Et}{ }_{3} \mathrm{~N}$ were added under nitrogen atmosphere. After the addition of (trimethylsilyl)acetylene $(7.59 \mathrm{~g}, 77.5 \mathrm{mmol})$, the mixture was stirred at $70{ }^{\circ} \mathrm{C}$ for $12 \mathrm{~h}$. After cooling down to room temperature, $200 \mathrm{~mL}$ of water was added. The mixture was extracted with $\mathrm{CH}_{2} \mathrm{Cl}_{2}$, and the organic layer was dried over anhydrous $\mathrm{Na}_{2} \mathrm{SO}_{4}$. After removal of solvent under vacuum, the residue was purified by silica gel chromatography with $\mathrm{CH}_{2} \mathrm{Cl}_{2}$ : methanol (100:0.5) as eluent to afford a yellow solid (5.0g, 80\%). ${ }^{1} \mathrm{H}$ NMR (600 MHz, $\left.\mathrm{CDCl}_{3}\right) \delta 8.76-8.71\left(\mathrm{~m}, 4 \mathrm{H}, \mathrm{tpy}-H^{3^{\prime} 5^{\prime}}\right.$ and tpy- $\left.H^{6,6^{\prime \prime}}\right), 8.67\left(\mathrm{dd}, J=8.0,1.3 \mathrm{~Hz}, 2 \mathrm{H}, \mathrm{tpy}-H^{3,3^{\prime \prime}}\right)$, $7.89\left(\mathrm{tt}, J=6.0,3.0 \mathrm{~Hz}, 2 \mathrm{H}, \mathrm{tpy}-H^{4,4 "}\right), 7.86\left(\mathrm{dd}, J=8.4,1.4 \mathrm{~Hz}, 2 \mathrm{H}, \mathrm{Ph}-H^{\mathrm{m}}\right), 7.63-7.58(\mathrm{~m}$, $\left.2 \mathrm{H} \mathrm{Ph}-H^{\mathrm{n}}\right), 7.36\left(\mathrm{ddt}, J=7.5,4.8,1.4 \mathrm{~Hz}, 2 \mathrm{H}, \mathrm{tpy}-H^{5,5 "}\right), 0.28\left(\mathrm{~s}, 9 \mathrm{H}, H^{\mathrm{o}}\right) .{ }^{13} \mathrm{C} \mathrm{NMR}(150$ $\left.\mathrm{MHz}, \mathrm{CDCl}_{3}\right) \delta 156.2,156.1,149.2,138.5,137.0,132.6,127.2,124.0,121.5,118.7,104.8$, 95.9, 0.1. ESI-MS (m/z): Calcd. for $\left[\mathrm{C}_{26} \mathrm{H}_{23} \mathrm{~N}_{3} \mathrm{Si}+\mathrm{H}\right]^{+}: 406.17$. Found: 406.13.

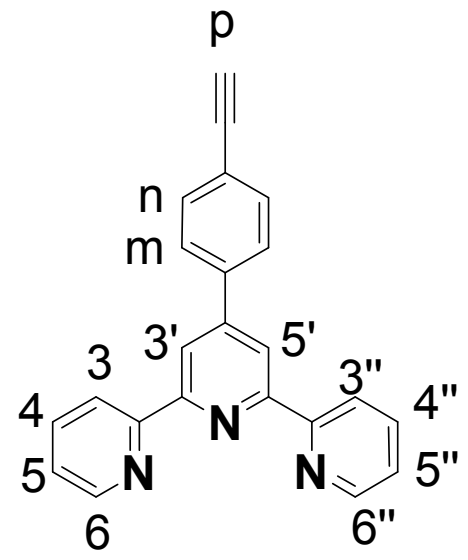

Compound 8. Compound 8 was synthesized using the reported method. ${ }^{2-3}$ To a flask containing solution of compound $7(3.5 \mathrm{~g}, 8.64 \mathrm{mmol})$ in $\mathrm{CHCl}_{3}(17 \mathrm{~mL})$ and $\mathrm{CH}_{3} \mathrm{OH}(50$ $\mathrm{mL}), \mathrm{K}_{2} \mathrm{CO}_{3}(5.96 \mathrm{~g}, 43 \mathrm{mmol})$ was added. After being stirred at room temperature for $4 \mathrm{~h}$, the mixture was extracted with $\mathrm{CHCl}_{3}$ and further purified by column chromatography on silica gel with chloroform: methanol (100:0.5) as eluent to afford the product as a light-yellow solid (2.6 g, 90\%). ${ }^{1} \mathrm{H}$ NMR (600 MHz, $\left.\mathrm{CDCl}_{3}\right) \delta 8.74$ (s, 2H, tpy- $\left.H^{3^{\prime}, 5^{\prime}}\right), 8.73$ (m, $\left.2 \mathrm{H}, \operatorname{tpy}-H^{6,6 "}\right), 8.67\left(\mathrm{dt}, J=7.9,1.1 \mathrm{~Hz}, 2 \mathrm{H}, \operatorname{tpy}-H^{3,3 "}\right), 7.88\left(\mathrm{~m}, 4 \mathrm{H}, \mathrm{tpy}-H^{4,4^{\prime \prime}}\right.$ and $\left.\mathrm{Ph}-H^{\mathrm{m}}\right)$, $7.67-7.61\left(\mathrm{~m}, 2 \mathrm{H}, \mathrm{Ph}-H^{\mathrm{n}}\right), 7.36\left(\mathrm{ddd}, J=7.5,4.8,1.2 \mathrm{~Hz}, 2 \mathrm{H}, \mathrm{tpy}-H^{5,5^{\prime \prime}}\right), 3.18\left(\mathrm{~s}, 1 \mathrm{H}, H^{\mathrm{p}}\right)$. ${ }^{13} \mathrm{C}$ NMR $\left(150 \mathrm{MHz}, \mathrm{CDCl}_{3}\right) \delta 156.2,156.2,149.4,149.3,138.9,137.0,132.8,127.4,124.0$, 122.9, 121.5, 118.8, 83.5, 78.7. ESI-MS $(\mathrm{m} / \mathrm{z})$ : Calcd. for $\left[\mathrm{C}_{23} \mathrm{H}_{15} \mathrm{~N}_{3}+\mathrm{H}\right]^{+}$: 334.39. Found: 334.05 . 


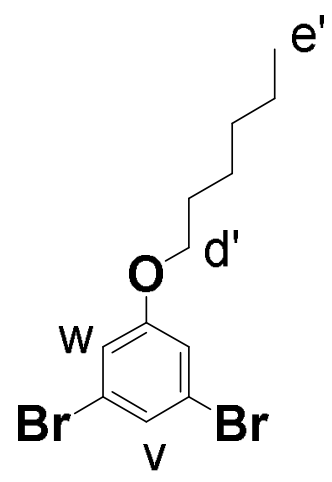

Compound 9. Compound 9 was synthesized using the reported method. ${ }^{3}$ 3,5-dibromophenol $(6.9 \mathrm{~g}, 27.6 \mathrm{mmol})$ and $\mathrm{K}_{2} \mathrm{CO}_{3}(7.62 \mathrm{~g}, 55.2 \mathrm{mmol})$ were added into a $200 \mathrm{~mL}$ Schlenk flask and degassed for three times. 1-Bromohexane $(6.79 \mathrm{~g}, 41.4 \mathrm{mmol})$ and DMF (50 mL) were added under $\mathrm{N}_{2}$. The solution was stirred at $85{ }^{\circ} \mathrm{C}$ for $24 \mathrm{~h}$ under $\mathrm{N}_{2}$. After that, the mixture was cooled to room temperature. Then $200 \mathrm{~mL}$ distilled water was added and extracted with $\mathrm{CH}_{2} \mathrm{Cl}_{2}$. The combined organic phase was washed with brine and dried with anhydrous $\mathrm{Na}_{2} \mathrm{SO}_{4}$. After concentrated by rotary evaporator, the crude product was purified by silica gel chromatography with hexanes $(8.3 \mathrm{~g}, 90 \%) .{ }^{1} \mathrm{H} \mathrm{NMR}\left(600 \mathrm{MHz}, \mathrm{CDCl}_{3}\right) \delta 7.22(\mathrm{t}, J=1.6 \mathrm{~Hz}$, $\left.1 \mathrm{H}, \mathrm{Ph}-H^{\mathrm{v}}\right), 6.98\left(\mathrm{~d}, J=1.6 \mathrm{~Hz}, 2 \mathrm{H}, \mathrm{Ph}-H^{\mathrm{w}}\right), 3.91\left(\mathrm{t}, J=6.5 \mathrm{~Hz}, 2 \mathrm{H}, \mathrm{Ph}-H^{\mathrm{d}^{\prime}}\right), 1.79-1.74(\mathrm{~m}$, 2H, Alkyl-H), $1.47-1.42$ (m, 2H, Alkyl- $H$ ), 1.33 (m, 4H, Alkyl- $H$ ), $0.92-0.88$ (m, 3H, Alkyl- $\left.H^{\mathrm{e}^{\prime}}\right) .{ }^{13} \mathrm{C} \mathrm{NMR}\left(150 \mathrm{MHz}, \mathrm{CDCl}_{3}\right) \delta 160.4,126.2,123.2,117.0,68.7,31.6,29.1,25.7$, $22.7,14.2$.

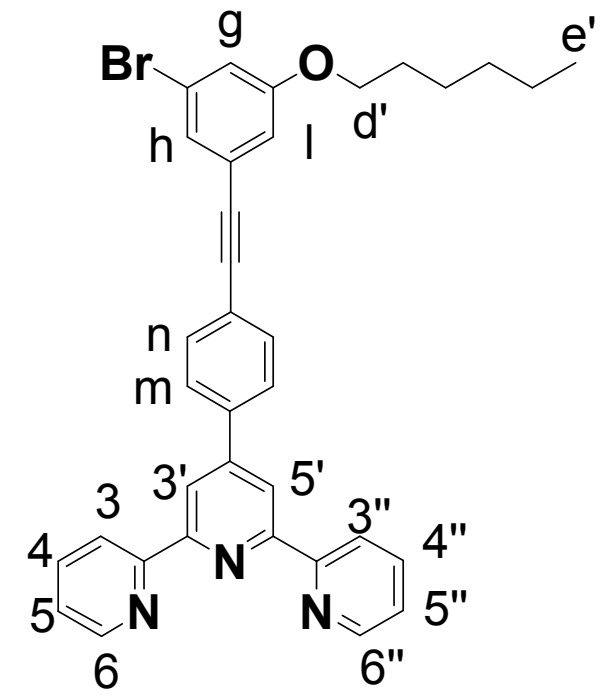

Compound 10. A mixture of compound 8 (2.3 g, $6.9 \mathrm{mmol})$, compound 9 (6.9 g, $20.7 \mathrm{mmol})$, $\mathrm{Pd}\left(\mathrm{PPh}_{3}\right)_{4}(398 \mathrm{mg}, 0.345 \mathrm{mmol})$, and $\mathrm{CuI}(39.4 \mathrm{mg}, 0.207 \mathrm{mmol})$ was degassed under 
nitrogen for three times. After that, $40 \mathrm{~mL}$ anhydrous THF and $20 \mathrm{~mL}$ anhydrous $\mathrm{Et}_{3} \mathrm{~N}$ were added subsequently. The mixture was stirred under $70{ }^{\circ} \mathrm{C}$ overnight. After cooling down to room temperature, solvent was removed under vacuum, and the residue was extracted with $\mathrm{CH}_{2} \mathrm{Cl}_{2}$. The combined organic phase was washed with brine and dried over $\mathrm{Na}_{2} \mathrm{SO}_{4}$. After removal of solvent under vacuum, the residue was purified by silica gel chromatography with $\mathrm{CH}_{2} \mathrm{Cl}_{2}$ : methanol (100:0.5) as eluent to afford a yellow solid (2.1 g, 51.8\%). ${ }^{1} \mathrm{H}$ NMR (600 $\left.\mathrm{MHz}, \mathrm{CDCl}_{3}\right) \delta 8.76\left(\mathrm{~s}, 2 \mathrm{H}, \mathrm{tpy}-H^{3^{\prime}, 5^{\prime}}\right), 8.75\left(\mathrm{ddd}, J=4.8,1.8,0.9 \mathrm{~Hz}, 2 \mathrm{H}\right.$, tpy- $\left.H^{6,6 "}\right), 8.69$ (dd, $\left.J=7.9,1.1 \mathrm{~Hz}, 2 \mathrm{H}, \operatorname{tpy}-H^{3,3 "}\right), 7.94-7.92\left(\mathrm{~m}, 2 \mathrm{H}, \mathrm{Ph}-H^{\mathrm{m}}\right), 7.90(\mathrm{td}, J=7.7,1.8 \mathrm{~Hz}, 2 \mathrm{H}$, tpy- $\left.H^{4,4 "}\right), 7.66\left(\mathrm{~d}, J=8.3 \mathrm{~Hz}, 2 \mathrm{H}, \mathrm{Ph}-H^{\mathrm{n}}\right), 7.37$ (ddd, $J=7.4,4.7,1.2 \mathrm{~Hz}, 2 \mathrm{H}$, tpy- $\left.H^{5,5}\right), 7.29$ $\left(\mathrm{t}, J=1.5 \mathrm{~Hz}, 1 \mathrm{H}, \mathrm{Ph}-H^{\mathrm{l}}\right), 7.06\left(\mathrm{t}, \mathrm{J}=2.1 \mathrm{~Hz}, 1 \mathrm{H}, \mathrm{Ph}-H^{\mathrm{h}}\right), 7.02(\mathrm{dd}, J=2.3,1.3 \mathrm{~Hz}, 1 \mathrm{H}$, $\left.\mathrm{Ph}-H^{\mathrm{g}}\right), 3.97\left(\mathrm{t}, J=6.5 \mathrm{~Hz}, 2 \mathrm{H}\right.$, Alkyl- $\left.H^{\mathrm{d}}\right), 1.82-1.77$ (m, 2H, Alkyl- $\left.H\right), 1.49-1.44$ (m, 2H, Alkyl-H), 1.36 (m, 4H, Alkyl-H), $0.94-0.90$ (m, 3H, Alkyl- $\left.H^{\mathrm{e}^{\prime}}\right) .{ }^{13} \mathrm{C}$ NMR (150 MHz, $\left.\mathrm{CDCl}_{3}\right) \delta 159.6,156.0,155.9,149.0,148.9,138.3,136.7,132.2,127.2,126.6,125.4,123.8$, $123.4,122.5,121.3,118.6,118.4,116.1,90.1,89.4,68.4,31.5,29.0,25.6,22.6,14.1$. ESI-MS $(m / z)$ : Calcd. for $\left[\mathrm{C}_{35} \mathrm{H}_{30} \mathrm{BrN}_{3} \mathrm{O}+\mathrm{H}\right]^{+}:$588.16. Found: 588.08 .

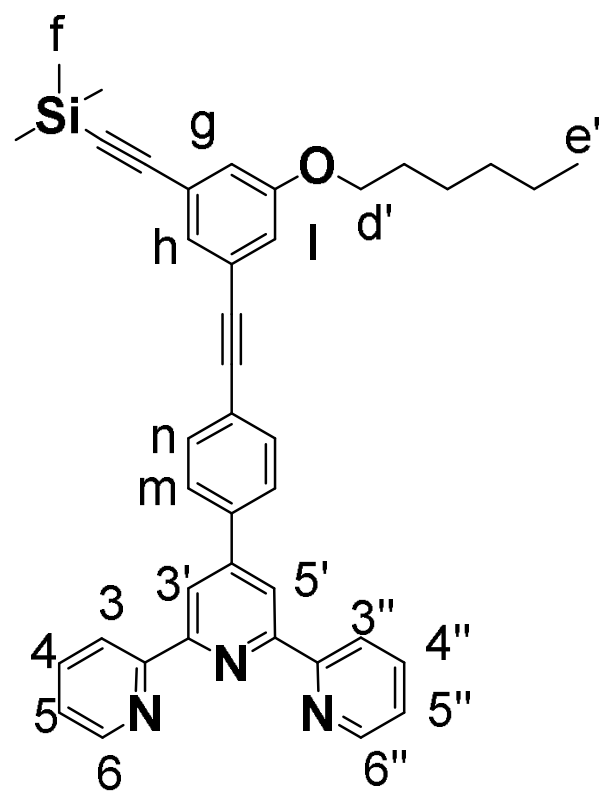

Compound 11. Compound 10 (1.87 g, $3.18 \mathrm{mmol}), \mathrm{Pd}\left(\mathrm{PPh}_{3}\right)_{4}(182.4 \mathrm{mg}, 0.158 \mathrm{mmol})$ and $\mathrm{CuI}$ (18 mg, $0.094 \mathrm{mmol})$ were mixed in a $100 \mathrm{~mL}$ Schlenk flask. After degassing and backfill with nitrogen for three times, $30 \mathrm{~mL}$ THF and $10 \mathrm{~mL} \mathrm{Et}_{3} \mathrm{~N}$ were added under 
nitrogen atmosphere. After the addition of (trimethylsilyl)acetylene (1.56 g, $15.9 \mathrm{mmol})$, the mixture was stirred at $70^{\circ} \mathrm{C}$. After cooling down to room temperature, $200 \mathrm{~mL}$ of water was added. The mixture was extracted with $\mathrm{CH}_{2} \mathrm{Cl}_{2}$, and the organic layer was dried over anhydrous $\mathrm{Na}_{2} \mathrm{SO}_{4}$. After removal of solvent under vacuum, the residue was purified by column chromatography on silica gel with DCM: methanol (100:0.7) as eluent to afford a yellow solid (1.7 g, 85\%). ${ }^{1} \mathrm{H}$ NMR $\left(600 \mathrm{MHz}, \mathrm{CDCl}_{3}\right) \delta 8.75\left(\mathrm{~s}, 2 \mathrm{H}, \mathrm{tpy}-H^{3^{\prime}, 5^{\prime}}\right), 8.74$ (ddd, $J$ $=4.7,1.8,0.9 \mathrm{~Hz}, 2 \mathrm{H}$, tpy- $\left.H^{6,6 "}\right), 8.68\left(\mathrm{~d}, J=7.8 \mathrm{~Hz}, 2 \mathrm{H}\right.$, tpy- $\left.H^{3,3 "}\right), 7.92-7.90(\mathrm{~m}, 2 \mathrm{H}$, $\left.\mathrm{Ph}-H^{\mathrm{m}}\right), 7.88\left(\mathrm{td}, J=7.7,1.7 \mathrm{~Hz}, 2 \mathrm{H}, \mathrm{tpy}-H^{4,4 "}\right), 7.66-7.63\left(\mathrm{~m}, 2 \mathrm{H}, \mathrm{Ph}-H^{\mathrm{n}}\right), 7.36(\mathrm{ddd}, J=$ 7.4, 4.7, $\left.1.1 \mathrm{~Hz}, 2 \mathrm{H}, \mathrm{tpy}-H^{5,5 "}\right), 7.28$ (t, $\left.J=1.4 \mathrm{~Hz}, 1 \mathrm{H}, \mathrm{Ph}-H^{\mathrm{l}}\right), 7.05$ (dd, $J=2.5,1.4 \mathrm{~Hz}, 1 \mathrm{H}$, $\left.\mathrm{Ph}-H^{\mathrm{h}}\right), 6.98\left(\mathrm{dd}, J=2.6,1.3 \mathrm{~Hz}, 1 \mathrm{H}, \mathrm{Ph}-H^{\mathrm{g}}\right), 3.97\left(\mathrm{t}, J=6.5 \mathrm{~Hz}, 2 \mathrm{H}, \mathrm{Alkyl}-H^{\mathrm{d}}\right), 1.82-1.76$ (m, 2H, Alkyl-H), $1.49-1.43$ (m, 2H, Alkyl- $H), 1.37$ - 1.33 (m, 4H, Alkyl- $H$ ), 0.92 (ddt, $J=$ 7.9, 4.5, 3.0 Hz, 3H, Alkyl- $\left.H^{\mathrm{e}^{\prime}}\right), 0.26$ (s, 9H, $\left.H^{\mathrm{f}}\right) .{ }^{13} \mathrm{C} \mathrm{NMR}\left(100 \mathrm{MHz}, \mathrm{CDCl}_{3}\right) \delta 158.8,156.2$, $156.1,149.3,149.2,138.4,136.9,132.3,127.7,127.3,124.4,124.3,124.0,123.8,121.4$, $118.7,118.3,118.2,104.3,94.7,90.2,89.4,68.4,31.6,29.2,25.8,22.7,14.2,0.0$. ESI-MS $(m / z)$ : Calcd. for $\left[\mathrm{C}_{40} \mathrm{H}_{39} \mathrm{~N}_{3} \mathrm{OSi}+\mathrm{H}\right]^{+}:$606.29. Found: 606.22.

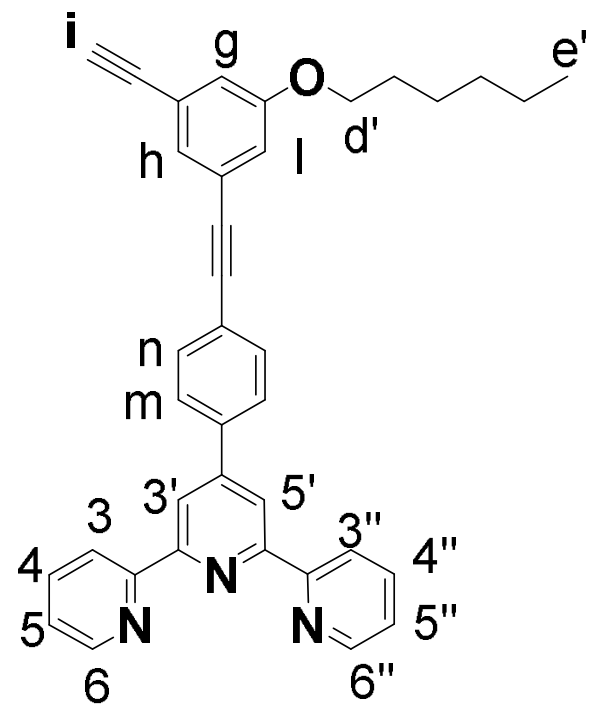

Compound 12. To a flask containing solution of compound $\mathbf{1 1}(1.2 \mathrm{~g}, 1.98 \mathrm{mmol})$ in $\mathrm{CHCl}_{3}$ $(17 \mathrm{~mL})$ and $\mathrm{CH}_{3} \mathrm{OH}(50 \mathrm{~mL}), \mathrm{K}_{2} \mathrm{CO}_{3}(5.96 \mathrm{~g}, 43 \mathrm{mmol})$ was added. After being stirred at room temperature for $4 \mathrm{~h}$, the mixture was extracted with $\mathrm{CHCl}_{3}$ and further purified by column chromatography on silica gel with chloroform: methanol (100:0.5) as eluent to afford the product as a light-yellow solid (0.95 g, 90\%). ${ }^{1} \mathrm{H}$ NMR (600 MHz, $\left.\mathrm{CDCl}_{3}\right) \delta 8.77(\mathrm{~s}, 2 \mathrm{H}$, 
tpy- $\left.H^{3^{\prime}, 5^{\prime}}\right), 8.75\left(\mathrm{dd}, J=5.0,1.6 \mathrm{~Hz}, 2 \mathrm{H}, \mathrm{tpy}-H^{6,6^{\prime \prime}}\right), 8.69\left(\mathrm{~d}, J=7.9 \mathrm{~Hz}, 2 \mathrm{H}, \mathrm{tpy}-H^{3,3 "}\right), 7.92(\mathrm{~d}$, $\left.J=8.1 \mathrm{~Hz}, 2 \mathrm{H}, \mathrm{Ph}-H^{\mathrm{m}}\right), 7.91-7.88\left(\mathrm{~m}, 2 \mathrm{H}, \mathrm{tpy}-H^{4,4 "}\right), 7.67$ (d, $\left.J=8.2 \mathrm{~Hz}, 2 \mathrm{H}, \mathrm{Ph}-H^{\mathrm{n}}\right), 7.37$ (ddd, $\left.J=7.4,4.7,1.2 \mathrm{~Hz}, 2 \mathrm{H}, \operatorname{tpy}-H^{5,5 "}\right), 7.29$ (s, $\left.1 \mathrm{H}, \mathrm{Ph}-H^{\mathrm{l}}\right), 7.08(\mathrm{dd}, J=2.5,1.4 \mathrm{~Hz}, 1 \mathrm{H}$, $\left.\mathrm{Ph}-H^{\mathrm{h}}\right), 7.01\left(\mathrm{dd}, J=2.6,1.3 \mathrm{~Hz}, 1 \mathrm{H}, \mathrm{Ph}-H^{\mathrm{g}}\right), 3.98\left(\mathrm{t}, J=6.5 \mathrm{~Hz}, 2 \mathrm{H}\right.$, Alkyl- $\left.H^{\mathrm{d}}\right), 3.08(\mathrm{~s}, 1 \mathrm{H}$, $\left.H^{\mathrm{i}}\right), 1.79(\mathrm{dd}, J=8.5,6.5 \mathrm{~Hz}, 2 \mathrm{H}$, Alkyl- $H), 1.46$ (m, 2H, Alkyl- $\left.H\right), 1.35$ (m, 4H, Alkyl- $H$ ), $0.94-0.90\left(\mathrm{~m}, 3 \mathrm{H}\right.$, Alkyl- $\left.H^{\mathrm{e}^{\prime}}\right) .{ }^{13} \mathrm{C} \mathrm{NMR}\left(150 \mathrm{MHz}, \mathrm{CDCl}_{3}\right) \delta 158.9,156.2,156.2,149.4$, $149.3,138.5,137.0,132.3,127.8,127.4,124.5,124.0,123.8,123.5,121.5,118.8,118.3,90.0$, 89.6, 83.0, 77.6, 68.4, 31.7, 29.2, 25.8, 22.7, 14.2. ESI-MS $(\mathrm{m} / \mathrm{z})$ : Calcd. for $\left[\mathrm{C}_{37} \mathrm{H}_{31} \mathrm{~N}_{3} \mathrm{O}+\mathrm{H}\right]^{+}$: 534.25. Found: 534.17.

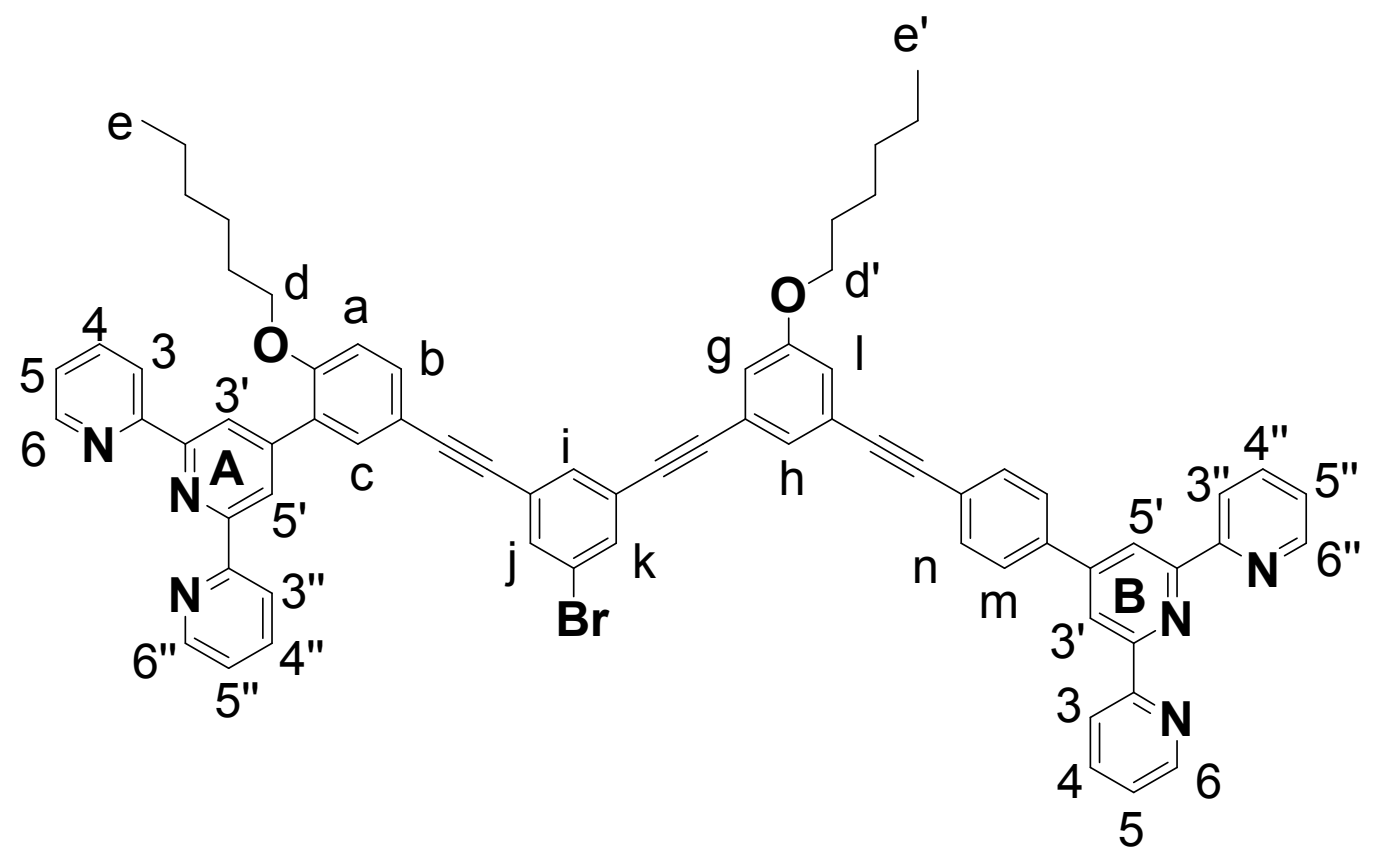

Compound 13. Compound 5 (3.9 g, $5.89 \mathrm{mmol})$, compound 12 (1.0 g, $1.96 \mathrm{mmol})$, $\mathrm{Pd}\left(\mathrm{PPh}_{3}\right)_{4}(113 \mathrm{mg}, 0.098 \mathrm{mmol})$ and $\mathrm{CuI}(11.2 \mathrm{mg}, 0.0588 \mathrm{mmol})$ were mixed in a $200 \mathrm{~mL}$ Schlenk flask. After degassing and backfill with nitrogen for three times, $30 \mathrm{~mL}$ DMF, $30 \mathrm{~mL}$ DME, and $50 \mathrm{~mL} \mathrm{Et}_{3} \mathrm{~N}$ were added under nitrogen atmosphere. The mixture was stirred at $70{ }^{\circ} \mathrm{C}$ for $12 \mathrm{~h}$. After cooling down to room temperature, $200 \mathrm{~mL}$ of water was added. The mixture was extracted with $\mathrm{CH}_{2} \mathrm{Cl}_{2}$, and the organic layer was dried over anhydrous $\mathrm{Na}_{2} \mathrm{SO}_{4}$. After removal of solvent under vacuum, the residue was purified by silica gel chromatography with $\mathrm{CH}_{2} \mathrm{Cl}_{2}$ : methanol (100:1.5) as eluent to afford a yellow solid (1.3 g, 65\%). ${ }^{1} \mathrm{H}$ NMR (600 MHz, $\left.\mathrm{CDCl}_{3}\right) 8.77$ (s, 2H, tpy $\left.{ }^{\mathrm{A}}-H^{3^{\prime}, 5^{\prime}}\right), 8.74(\mathrm{dd}, J=5.1,1.7 \mathrm{~Hz}, 2 \mathrm{H}$, $\left.\operatorname{tpy}^{\mathrm{A}}-H^{6,6^{\prime \prime}}\right), 8.73-8.70\left(\mathrm{~m}, 4 \mathrm{H}, \operatorname{tpy}^{\mathrm{B}}-H^{6,6^{\prime \prime}}\right.$ and tpy $\left.{ }^{\mathrm{B}}-H^{3^{\prime}, 5^{\prime}}\right), 8.68\left(\mathrm{~d}, J=8.0 \mathrm{~Hz}, 4 \mathrm{H}, \mathrm{tpy}^{\mathrm{A}}-H^{3,3^{\prime \prime}}\right.$, 
$\left.\operatorname{tpy}^{\mathrm{B}}-H^{3,3 "}\right), 7.95-7.84\left(\mathrm{~m}, 6 \mathrm{H}, \mathrm{Ph}-H^{\mathrm{m}}\right.$, tpy $^{\mathrm{A}}-H^{4,4^{\prime \prime}}$, tpy $\left.^{\mathrm{B}}-H^{4,4 "}\right), 7.78\left(\mathrm{~d}, J=2.2 \mathrm{~Hz}, 1 \mathrm{H}, \mathrm{Ph}-H^{\mathrm{c}}\right)$, 7.70-7.60 (m, 5H, Ph- $\left.H^{\mathrm{n}}, \mathrm{Ph}-H^{\mathrm{h}}, \mathrm{Ph}-H^{\mathrm{j}}, \mathrm{Ph}-H^{\mathrm{k}}\right), 7.54\left(\mathrm{dd}, J=8.5,2.2 \mathrm{~Hz}, 1 \mathrm{H}, \mathrm{Ph}-H^{\mathrm{b}}\right), 7.39-$ $7.31\left(\mathrm{~m}, 5 \mathrm{H}, \mathrm{tpy}^{\mathrm{A}}-H^{5,5^{\prime \prime}}, \mathrm{tpy}^{\mathrm{B}}-H^{5,5^{\prime \prime}}, \mathrm{Ph}-H^{\mathrm{i}}\right), 7.09\left(\mathrm{t}, J=1.8 \mathrm{~Hz}, 1 \mathrm{H}, \mathrm{Ph}-H^{\mathrm{l}}\right), 7.04(\mathrm{t}, J=1.8 \mathrm{~Hz}$, $\left.1 \mathrm{H}, \mathrm{Ph}-H^{\mathrm{g}}\right), 6.99\left(\mathrm{~d}, J=8.6 \mathrm{~Hz}, 1 \mathrm{H}, \mathrm{Ph}-H^{\mathrm{a}}\right), 4.05\left(\mathrm{t}, J=6.2 \mathrm{~Hz}, 2 \mathrm{H}, \mathrm{Ph}-H^{\mathrm{d}}\right), 4.00(\mathrm{t}, J=6.5$ $\left.\mathrm{Hz}, 2 \mathrm{H}, \mathrm{Ph}-H^{\mathrm{d}}\right), 1.84-1.78$ (m, 2H, Alkyl- $\left.H\right), 1.80-1.71$ (m, 2H, Alkyl-H), 1.48 (m, 2H, Alkyl-H), 1.47-1.31 (m, 6H, Alkyl-H), 1.23-1.08 (m, 4H, Alkyl-H), $0.95-0.89$ (m, 3H, Alkyl- $H), 0.74(\mathrm{t}, J=7.2 \mathrm{~Hz}, 3 \mathrm{H}$, Alkyl- $H) .{ }^{13} \mathrm{C} \mathrm{NMR}\left(150 \mathrm{MHz}, \mathrm{CDCl}_{3}\right) \delta 159.5,156.9$, $156.5,156.3,156.0,155.3,149.7,149.2,149.2$, 147.5, 142.1, 140.8, 137.8, 137.0, 136.9, $134.2,133.9,133.7,133.6,133.2,128.7,127.9,127.7,125.8,125.2,124.0,124.0,123.8$, $123.0,122.0,121.9,121.5,121.3,118.8,115.9$, 115.1, 114.8, 112.2, 91.4, 91.3, 87.3, 86.5, 68.8, 68.4, 31.7, 31.6, 29.3, 29.2, 25.9, 25.9, 22.8, 22.5, 14.2, 14.1. ESI-MS ( $\mathrm{m} / \mathrm{z})$ : Calcd. for $\left[\mathrm{C}_{72} \mathrm{H}_{59} \mathrm{BrN}_{6} \mathrm{O}_{2}+\mathrm{H}\right]^{+}:$1119.39, $\left[\mathrm{C}_{72} \mathrm{H}_{59} \mathrm{BrN}_{6} \mathrm{O}_{2}+2 \mathrm{H}\right]^{2+}: 560.19$. Found: $1119.30(1+), 560.13$ $(2+)$.

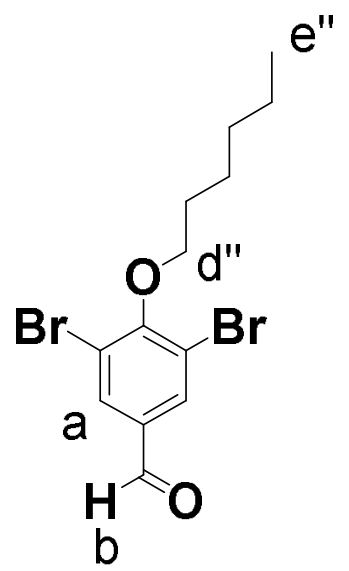

Compound 14. Compound 14 was synthesized according to literature. ${ }^{4}$ To a solution of 3,5-dibromo-4-hydroxybenzaldehyde (5.0 g, $13.8 \mathrm{mmol})$ in DMF $(50 \mathrm{~mL}), 1$-bromohexane (4.52 $\mathrm{g}, 27.6 \mathrm{mmol}), \mathrm{K}_{2} \mathrm{CO}_{3}(5.7 \mathrm{~g}, 41.4 \mathrm{mmol})$ were added and the mixture was heated at $85{ }^{\circ} \mathrm{C}$ overnight. After cooling to room temperature, the mixture was extracted with $\mathrm{CH}_{2} \mathrm{Cl}_{2}$ and further purified by silica gel column chromatography with $\mathrm{CH}_{2} \mathrm{Cl}_{2}$ : hexane (1:4) to afford the product as light-yellow solid (5.8 g, 75\%). ${ }^{1} \mathrm{H}$ NMR $\left(600 \mathrm{MHz}, \mathrm{CDCl}_{3}\right) \delta 9.85\left(\mathrm{~s}, 1 \mathrm{H}, H^{\mathrm{b}}\right)$, $8.02\left(\mathrm{~s}, 2 \mathrm{H}, \mathrm{Ph}-H^{\mathrm{a}}\right), 4.09\left(\mathrm{t}, J=6.6 \mathrm{~Hz}, 2 \mathrm{H}\right.$, Alkyl- $\left.H^{\mathrm{d}}\right), 1.94-1.86(\mathrm{~m}, 2 \mathrm{H}$, Alkyl- $H), 1.54$ $(\mathrm{m}, 2 \mathrm{H}$, Alkyl- $H), 1.37$ (m, 4H, Alkyl- $H), 0.95-0.90$ (m, 3H, Alkyl- $\left.H^{\mathrm{e}}\right) .{ }^{13} \mathrm{C}$ NMR (150 $\left.\mathrm{MHz}, \mathrm{CDCl}_{3}\right) \delta 188.6,158.7,134.1,134.0,119.6,74.3,31.7,30.1,25.6,22.7,14.2$. 


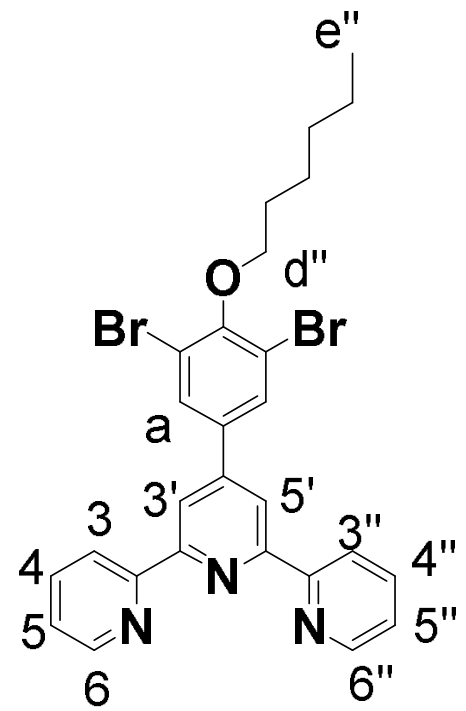

Compound 15. Compound 15 was synthesized according to literature. ${ }^{4}$ To a solution of $\mathrm{NaOH}$ powder $(10 \mathrm{~g}, 250 \mathrm{mmol})$ in $130 \mathrm{~mL}$ of $\mathrm{EtOH}$, compound $14(13 \mathrm{~g}, 45.5 \mathrm{mmol})$ and 2-acetylpyridine $(13.3 \mathrm{~g}, 110 \mathrm{mmol})$ were added. After stirring at $25{ }^{\circ} \mathrm{C}$ for $10 \mathrm{~h}$, aqueous $\mathrm{NH}_{3} \cdot \mathrm{H}_{2} \mathrm{O}(240 \mathrm{~mL})$ was added and the mixture was refluxed for $20 \mathrm{~h}$. After cooling to room temperature, the solid was collected by suction filtration and washed with cold ethanol to give the product as a white solid $(13 \mathrm{~g}, 60 \%)$ was obtained. ${ }^{1} \mathrm{H}$ NMR $\left(600 \mathrm{MHz}, \mathrm{CDCl}_{3}\right) \delta$ $8.74\left(\mathrm{dd}, J=4.7,1.6 \mathrm{~Hz}, 2 \mathrm{H}, \mathrm{tpy}-H^{6,6 "}\right), 8.67$ (dd, $\left.J=8.0,1.1 \mathrm{~Hz}, 2 \mathrm{H}, \mathrm{tpy}-H^{3,3 "}\right), 8.64$ (s, 2H, tpy- $\left.H^{3^{\prime}, 5^{\prime}}\right), 8.04\left(\mathrm{~s}, 2 \mathrm{H}, \mathrm{Ph}-H^{\mathrm{a}}\right), 7.89\left(\mathrm{td}, J=7.7,1.8 \mathrm{~Hz}, 2 \mathrm{H}, \mathrm{tpy}-H^{4,4^{\prime \prime}}\right), 7.37$ (ddd, $J=7.4,4.7$, $1.1 \mathrm{~Hz}, 2 \mathrm{H}$, tpy- $\left.H^{5,5 "}\right), 4.08$ (t, $J=6.6 \mathrm{~Hz}, 2 \mathrm{H}$, Alkyl- $\left.H^{\mathrm{d}}\right), 1.91$ (p, $J=6.9 \mathrm{~Hz}, 2 \mathrm{H}$, Alkyl- $H$ ), 1.69 (m, 2H, Alkyl- $H), 1.40$ (m, 4H, Alkyl- $H), 0.93$ (t, $J=6.7 \mathrm{~Hz}, 3 \mathrm{H}$, Alkyl- $\left.H^{\mathrm{e}}\right)$ ). ${ }^{13} \mathrm{C}$ NMR $\left(150 \mathrm{MHz} \mathrm{CDCl}_{3}\right) \delta 156.0,155.7,154.1,149.0,146.7,136.8,136.5,131.2,123.9,121.3$, 119.0, 118.2, 73.8, 31.7, 30.1, 25.6, 22.7, 14.2. ESI-MS (m/z): Calcd. for $\left[\mathrm{C}_{27} \mathrm{H}_{25} \mathrm{Br}_{2} \mathrm{~N}_{3} \mathrm{O}+\mathrm{H}\right]^{+}$: 566.04. Found: 565.96. 


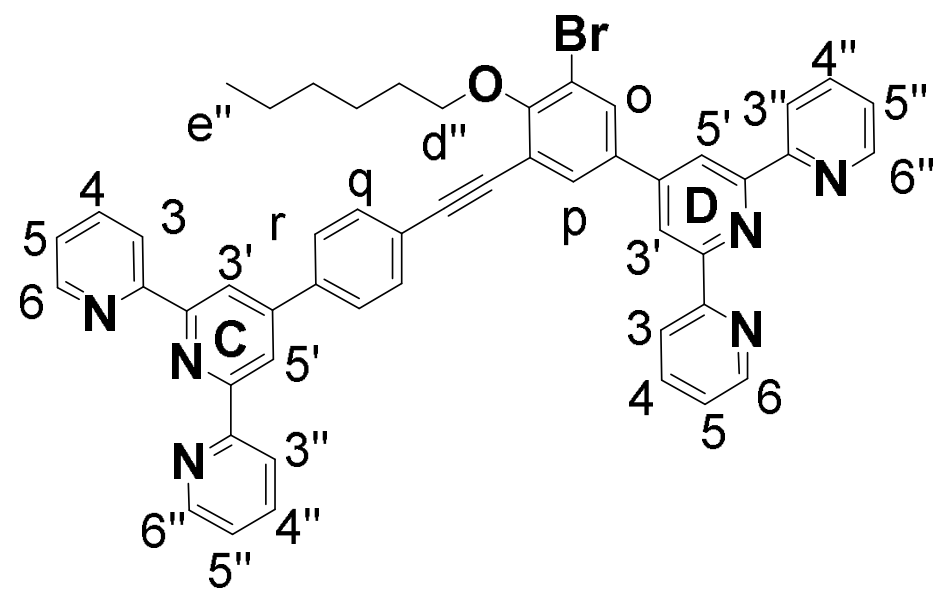

Compound 16. Compound 8 (1.0 g, $3 \mathrm{mmol})$, compound 15 (4.24 g, $7.5 \mathrm{mmol}), \mathrm{Pd}\left(\mathrm{PPh}_{3}\right)_{4}$ (173 mg, $0.15 \mathrm{mmol})$ and $\mathrm{CuI}(17.1 \mathrm{mg}, 0.09 \mathrm{mmol})$ were mixed in a $200 \mathrm{~mL}$ Schlenk flask. After degassing and backfill with nitrogen for three times, $20 \mathrm{~mL}$ DMF, $20 \mathrm{~mL}$ DME and 30 $\mathrm{mL} \mathrm{Et}{ }_{3} \mathrm{~N}$ were added under nitrogen atmosphere. The mixture was stirred at $70{ }^{\circ} \mathrm{C}$ for $12 \mathrm{~h}$. After cooling down to room temperature, $200 \mathrm{~mL}$ of water was added. The mixture was extracted with $\mathrm{CH}_{2} \mathrm{Cl}_{2}$, and the organic layer was dried over anhydrous $\mathrm{Na}_{2} \mathrm{SO}_{4}$. After removal of solvent under vacuum, the residue was purified by column chromatography on silica gel with DCM: methanol (100:1.5) as eluent to afford a yellow solid (1.5 g, 62.5\%). ${ }^{1} \mathrm{H}$ NMR (400 MHz, $\left.\mathrm{CDCl}_{3}\right) \delta 8.76\left(\mathrm{~s}, 2 \mathrm{H}, \operatorname{tpy}^{\mathrm{C}}-H^{3^{\prime} 5^{\prime}}\right), 8.75(\mathrm{ddd}, J=4.7,1.8,0.9 \mathrm{~Hz}, 2 \mathrm{H}$, $\left.\operatorname{tpy}^{\mathrm{C}}-H^{6,6^{\prime \prime}}\right), 8.73\left(\mathrm{ddd}, J=4.7,1.8,0.9 \mathrm{~Hz}, 2 \mathrm{H}, \operatorname{tpy}^{\mathrm{D}}-H^{6,6^{\prime \prime}}\right), 8.69\left(\mathrm{~s}, 2 \mathrm{H}\right.$, tpy $\left.^{\mathrm{D}}-H^{3^{\prime}, 5^{\prime}}\right), 8.67-8.66$ $\left(\mathrm{m}, 4 \mathrm{H}\right.$, tpy $^{\mathrm{C}}-H^{3,3^{\prime \prime}}$ and tpy $\left.{ }^{\mathrm{D}}-H^{3,3 "}\right), 8.11\left(\mathrm{~d}, J=2.2 \mathrm{~Hz}, 1 \mathrm{H}, \mathrm{Ph}-H^{\mathrm{p}}\right), 8.03(\mathrm{~d}, J=2.2 \mathrm{~Hz}$, $\left.1 \mathrm{H}, \mathrm{Ph}-H^{\mathrm{o}}\right), 7.96-7.94\left(\mathrm{~m}, 2 \mathrm{H}, \mathrm{Ph}-H^{\mathrm{r}}\right), 7.87\left(\mathrm{tt}, J=7.7,1.7 \mathrm{~Hz}, 4 \mathrm{H}, \operatorname{tpy}^{\mathrm{C}}-H^{4,4^{\prime \prime}}, \operatorname{tpy}^{\mathrm{D}}-H^{4,4 "}\right)$, $7.72-7.70\left(\mathrm{~m}, 2 \mathrm{H}, \mathrm{Ph}-H^{\mathrm{q}}\right), 7.36\left(\mathrm{dddd}, J=7.2,5.8,4.7,1.2 \mathrm{~Hz}, 4 \mathrm{H}, \operatorname{tpy}^{\mathrm{C}}-H^{5,5^{\prime \prime}}, \operatorname{tpy}^{\mathrm{D}}-H^{5,5^{\prime \prime}}\right)$, $4.29\left(\mathrm{t}, J=6.5 \mathrm{~Hz}, 2 \mathrm{H}\right.$, Alkyl- $\left.H^{\mathrm{d}}\right), 1.97-1.93(\mathrm{~m}, 2 \mathrm{H}$, Alkyl- $H), 1.64-1.59$ (m, 2H, Alkyl- $H$ ), 1.39 (m, 4H, Alkyl- $H), 0.92$ (t, $J=7.1 \mathrm{~Hz}, 3 \mathrm{H}$, Alkyl- $\left.H^{\mathrm{e}}\right) .{ }^{13} \mathrm{C}$ NMR $(150 \mathrm{MHz}$, $\left.\mathrm{CDCl}_{3}\right) \delta 158.0,156.2,156.2,156.1,149.4,149.3,147.9,138.7,137.1,137.0,135.3,132.3$, 132.3, 131.7, 127.5, 124.1, 124.0, 123.8, 121.5, 121.5, 121.5, 119.4, 118.8, 118.6, 118.5, 94.6, 86.6, 74.7, 31.9, 30.5, 26.0, 22.8, 14.3. ESI-MS $(m / z)$ : Calcd. for $\left[\mathrm{C}_{50} \mathrm{H}_{39} \mathrm{BrN}_{6} \mathrm{O}+\mathrm{H}\right]^{+}: 819.24$ and $\left[\mathrm{C}_{50} \mathrm{H}_{39} \mathrm{BrN}_{6} \mathrm{O}+2 \mathrm{H}\right]^{2+}: 410.12$. Found: 819.15 and 410.05 . 


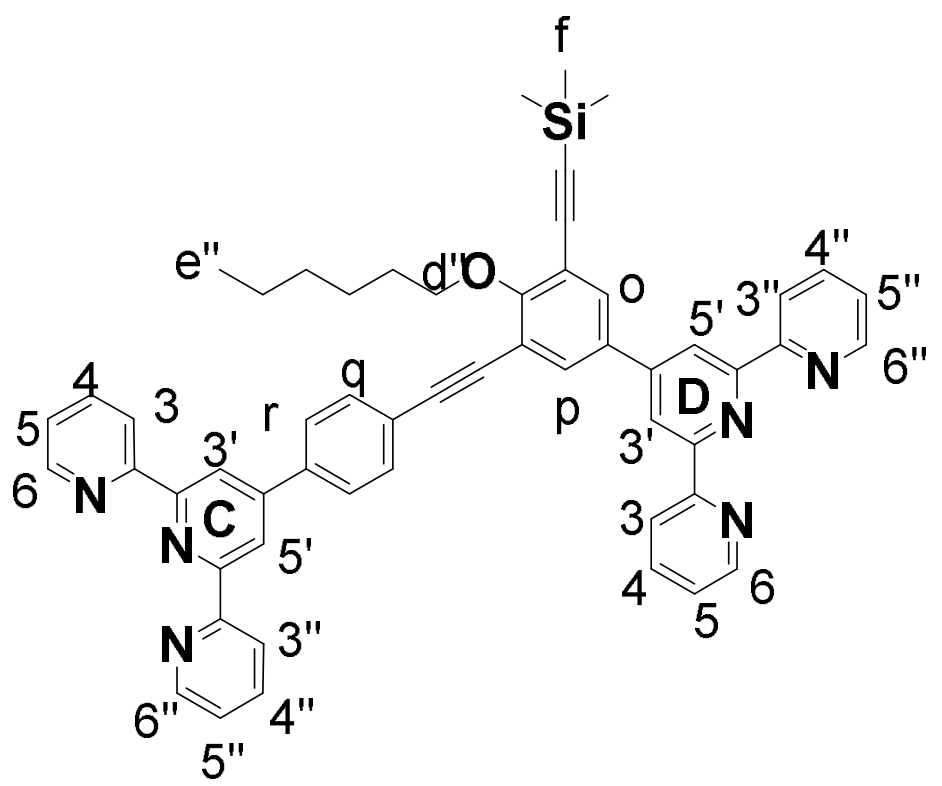

Compound 17. Compound 16 (1.5 g, $1.855 \mathrm{mmol}), \mathrm{Pd}\left(\mathrm{PPh}_{3}\right)_{4}(107 \mathrm{mg}, 0.0927 \mathrm{mmol})$, and $\mathrm{CuI}(10.59 \mathrm{mg}, 0.05565 \mathrm{mmol})$ were mixed in a $100 \mathrm{~mL}$ Schlenk flask. After degassing and backfill with nitrogen for three times, $30 \mathrm{~mL}$ THF and $10 \mathrm{~mL} \mathrm{Et}_{3} \mathrm{~N}$ were added under nitrogen atmosphere. After the addition of (trimethylsilyl)acetylene (0.9 g, $9.27 \mathrm{mmol})$, the mixture was stirred at $70{ }^{\circ} \mathrm{C}$ for $12 \mathrm{~h}$. After cooling down to room temperature, $200 \mathrm{~mL}$ of water was added. The mixture was extracted with $\mathrm{CH}_{2} \mathrm{Cl}_{2}$, and the organic layer was dried over anhydrous $\mathrm{Na}_{2} \mathrm{SO}_{4}$. After removal of solvent under vacuum, the residue was purified by silica gel chromatography with $\mathrm{CH}_{2} \mathrm{Cl}_{2}$ : methanol (100:1.5) as eluent to afford a yellow solid (1.1 g, 70\%). ${ }^{1} \mathrm{H}$ NMR (600 MHz, $\left.\mathrm{CDCl}_{3}\right) \delta 8.83\left(\mathrm{~s}, 2 \mathrm{H}\right.$, tpy $\left.^{\mathrm{C}}-H^{3^{\prime}, 5^{\prime}}\right), 8.77-8.73(\mathrm{~m}, 6 \mathrm{H}$, tpy $^{\mathrm{C}}-H^{6,6^{\prime \prime}}$, tpy $^{\mathrm{D}}-H^{6,6^{\prime \prime}}$, tpy $\left.^{\mathrm{D}}-H^{3^{\prime}, 5^{\prime}}\right), 8.69\left(\mathrm{t}, J=8.3 \mathrm{~Hz}, 4 \mathrm{H}\right.$, tpy $^{\mathrm{C}}-H^{3,3 "}$, tpy $\left.^{\mathrm{D}}-H^{3,3 "}\right), 8.05-8.01$ $\left(\mathrm{m}, 3 \mathrm{H}, \mathrm{Ph}-H^{\mathrm{p}}, \mathrm{Ph}-H^{\mathrm{o}}\right), 7.90\left(\mathrm{~m}, 6 \mathrm{H}, \mathrm{Ph}-H^{\mathrm{r}}, \mathrm{tpy}^{\mathrm{C}}-H^{4,4^{4}}, \mathrm{tpy}^{\mathrm{D}}-H^{4,4}\right), 7.80(\mathrm{~d}, J=8.1 \mathrm{~Hz}, 2 \mathrm{H}$, $\left.\mathrm{Ph}-H^{\mathrm{q}}\right), 7.39-7.35\left(\mathrm{~m}, 4 \mathrm{H}, \operatorname{tpy}^{\mathrm{C}}-H^{5,5^{\prime \prime}}, \operatorname{tpy}^{\mathrm{D}}-H^{5,5 "}\right), 3.86\left(\mathrm{t}, J=6.5 \mathrm{~Hz}, 2 \mathrm{H}\right.$, Alkyl- $\left.H^{\mathrm{d}}\right), 1.61$ $\left(\mathrm{m}, 2 \mathrm{H}\right.$, Alkyl-H), $1.33-1.16(\mathrm{~m}, 6 \mathrm{H}$, Alkyl- $H), 0.82$ (t, $J=7.1 \mathrm{~Hz}, 3 \mathrm{H}$, Alkyl- $\left.H^{\mathrm{e}}\right), 0.32$ (s, 9H, Alkyl- $\left.H^{\mathrm{f}}\right) .{ }^{13} \mathrm{C} \mathrm{NMR}\left(150 \mathrm{MHz}, \mathrm{CDCl}_{3}\right) \delta 159.1,156.3,156.1,156.0,149.9,149.2$, $149.2,148.9,138.6,137.6,137.0,137.0,135.6,134.1,132.2,130.1,127.2,124.0,123.9$, $121.5,121.4,118.8,118.6,101.3,99.4,74.3,31.7,30.3,25.7,25.63,22.7,14.2,0.1$. ESI-MS $(\mathrm{m} / z)$ : Calcd. for $\left[\mathrm{C}_{55} \mathrm{H}_{48} \mathrm{~N}_{6} \mathrm{OSi}+\mathrm{H}\right]^{+}: 837.37$ and $\left[\mathrm{C}_{55} \mathrm{H}_{48} \mathrm{~N}_{6} \mathrm{OSi}+2 \mathrm{H}\right]^{2+}:$ 419.15. Found: 837.27 and 419.11 . 


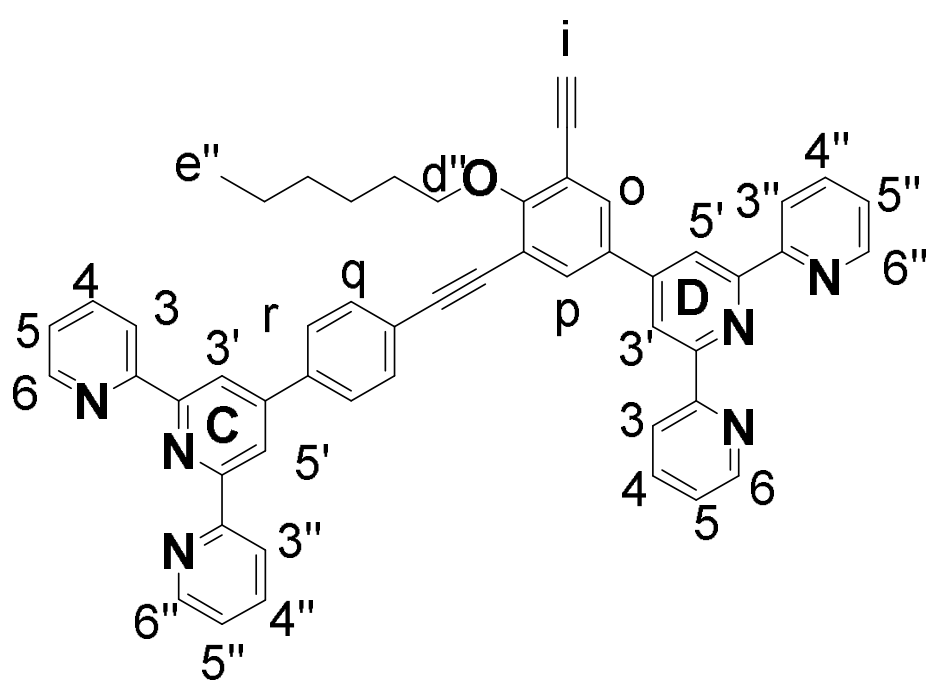

Compound 18. To a flask containing solution of compound 17 ( $0.8 \mathrm{~g}, 0.95 \mathrm{mmol})$ in $\mathrm{CHCl}_{3}$ $(15 \mathrm{~mL})$ and $\mathrm{MeOH}(45 \mathrm{~mL}), \mathrm{K}_{2} \mathrm{CO}_{3}(0.66 \mathrm{~g}, 4.78 \mathrm{mmol})$ was added. After being stirred at room temperature for $4 \mathrm{~h}$, the mixture was extracted with $\mathrm{CHCl}_{3}$ and further purified by column chromatography on silica gel with chloroform: methanol (100:0.5) as eluent to afford the product as a light-yellow solid $(0.68 \mathrm{~g}, 95 \%) .{ }^{1} \mathrm{H}$ NMR $\left(600 \mathrm{MHz}, \mathrm{CDCl}_{3}\right) \delta 8.78(\mathrm{~s}, 2 \mathrm{H}$, $\left.\operatorname{tpy}^{\mathrm{C}}-H^{3^{\prime}, 5^{\prime}}\right), 8.77-8.74\left(\mathrm{~m}, 4 \mathrm{H}\right.$, tpy $^{\mathrm{C}}-H^{6,6^{\prime \prime}}$, tpy $\left.^{\mathrm{D}}-H^{6,6^{\prime}}\right), 8.72\left(\mathrm{~s}, 2 \mathrm{H}, \operatorname{tpy}^{\mathrm{D}}-H^{3^{\prime}, 5^{\prime}}\right), 8.69(\mathrm{~d}, J=7.9$ $\left.\mathrm{Hz}, 4 \mathrm{H}, \operatorname{tpy}^{\mathrm{C}}-H^{3,3^{\prime}}, \operatorname{tpy}^{\mathrm{D}}-H^{3,3 "}\right), 8.10\left(\mathrm{~d}, J=2.4 \mathrm{~Hz}, 1 \mathrm{H}, \mathrm{Ph}-H^{\mathrm{p}}\right), 8.02(\mathrm{~d}, J=2.4 \mathrm{~Hz}, 1 \mathrm{H}$, $\left.\mathrm{Ph}-H^{0}\right), 7.96\left(\mathrm{~d}, J=8.3 \mathrm{~Hz}, 2 \mathrm{H}, \mathrm{Ph}-H^{\mathrm{r}}\right), 7.90\left(\mathrm{td}, J=7.7,1.8 \mathrm{~Hz}, 4 \mathrm{H}, \operatorname{tpy}^{\mathrm{C}}-H^{4,4^{\prime \prime}}, \operatorname{tpy}^{\mathrm{D}}-H^{4,4^{\prime \prime}}\right)$, $7.73\left(\mathrm{~d}, J=8.3 \mathrm{~Hz}, 2 \mathrm{H}, \mathrm{Ph}-H^{\mathrm{q}}\right), 7.38\left(\mathrm{ddt}, J=7.5,4.7,1.5 \mathrm{~Hz}, 4 \mathrm{H}, \mathrm{tpy}^{\mathrm{C}}-H^{5,5^{\prime \prime}}, \operatorname{tpy}^{\mathrm{D}}-H^{5,5^{\prime \prime}}\right)$, $4.41\left(\mathrm{t}, J=6.5 \mathrm{~Hz}, 2 \mathrm{H}\right.$, Alkyl- $\left.H^{\mathrm{c} "}\right), 3.36\left(\mathrm{~s}, 1 \mathrm{H}, H^{\mathrm{i}}\right), 1.95-1.88\left(\mathrm{~m}, 2 \mathrm{H}\right.$, Alkyl- $\left.H^{\mathrm{d}}\right), 1.62(\mathrm{t}, J$ $=7.5 \mathrm{~Hz}, 4 \mathrm{H}$, Alkyl- $H$ ), 1.38 (m, 4H, Alkyl- $H$ ), 0.91 (t, $J=7.0 \mathrm{~Hz}, 3 \mathrm{H}$, Alkyl- $\left.H^{\mathrm{e}}\right) .{ }^{13} \mathrm{C}$ NMR $\left(150 \mathrm{MHz} \mathrm{CDCl}_{3}\right) \delta 162.5,156.1,156.1,156.0,149.2,148.1,138.5,136.9,133.6,133.1$, $132.9,132.2$, 127.4, 124.0, 123.9, 121.4, 121.4, 118.6, 118.4, 118.3, 117.2, 94.2, 86.8, 82.3, 79.6, 74.8, 31.8, 30.5, 25.9, 22.8, 14.2. ESI-MS (m/z): Calcd. for $\left[\mathrm{C}_{52} \mathrm{H}_{40} \mathrm{~N}_{6} \mathrm{O}+\mathrm{H}\right]^{+}: 765.33$. Found: 765.23. 


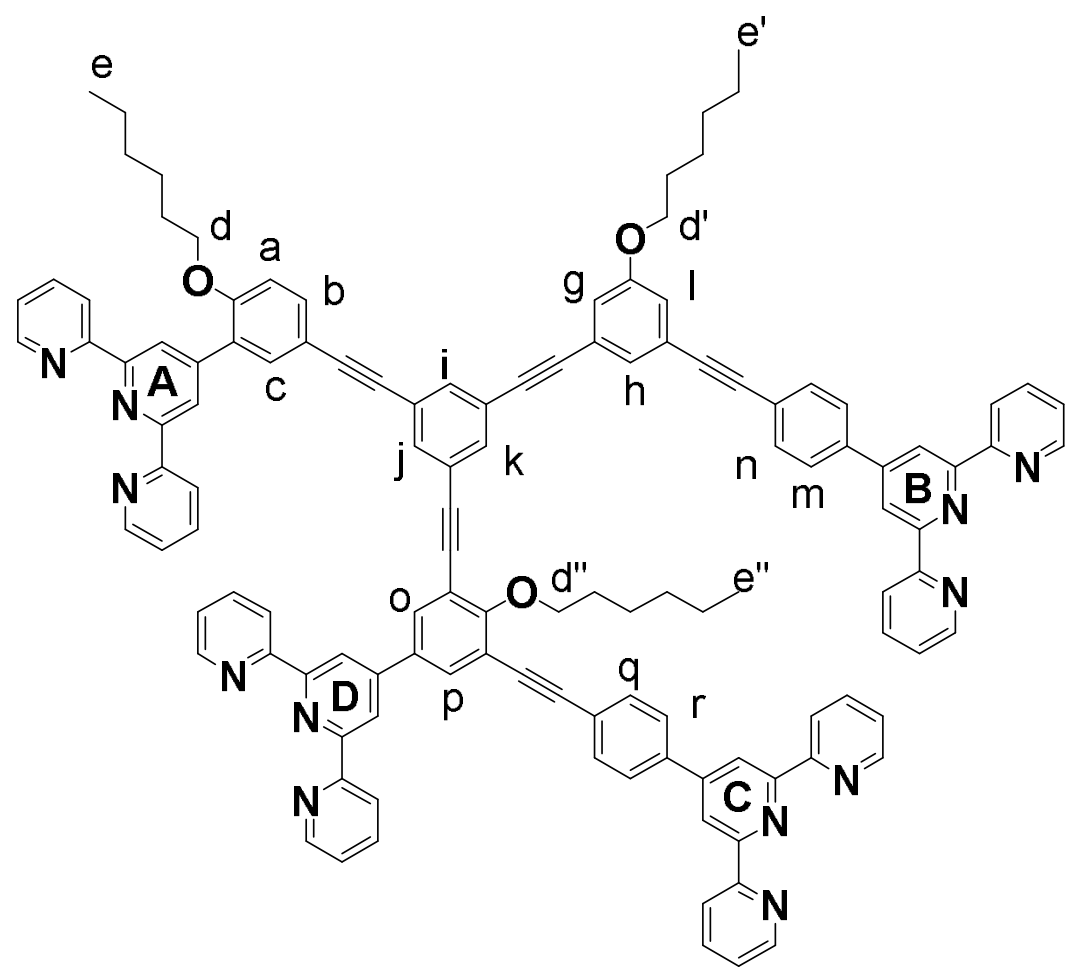

Ligand LA. Compound 13 (0.325 g, 0.29 mmol), compound 18 (0.148 g, 0.193 mmol), $\operatorname{Pd}\left(\mathrm{PPh}_{3}\right)_{4}(11.17 \mathrm{mg}, 0.098 \mathrm{mmol})$, and $\mathrm{CuI}(1.1 \mathrm{mg}, 0.058 \mathrm{mmol})$ were mixed in a $100 \mathrm{~mL}$ Schlenk flask. After degassing and backfill with nitrogen for three times, $10 \mathrm{~mL}$ DMF, $10 \mathrm{~mL}$ DME and $15 \mathrm{~mL} \mathrm{Et}_{3} \mathrm{~N}$ were added under nitrogen atmosphere. The mixture was stirred at $70{ }^{\circ} \mathrm{C}$ for $12 \mathrm{~h}$. After cooling down to room temperature, $100 \mathrm{~mL}$ of water was added. The mixture was extracted with $\mathrm{CH}_{2} \mathrm{Cl}_{2}$, and the organic layer was dried over anhydrous $\mathrm{Na}_{2} \mathrm{SO}_{4}$. After removal of solvent under vacuum, the residue was purified by column chromatography on silica gel with $\mathrm{CH}_{2} \mathrm{Cl}_{2}$ : methanol (100:3) as eluent to afford a yellow solid (150 mg, 40\%). ${ }^{1} \mathrm{H}$ NMR $\left(600 \mathrm{MHz}, \mathrm{CDCl}_{3}\right) \delta 8.78\left(\mathrm{~s}, 2 \mathrm{H}, \mathrm{tpy}^{\mathrm{D}}-H^{3^{\prime}, 5^{\prime}}\right), 8.76\left(\mathrm{~m}, 6 \mathrm{H}, \mathrm{tpy}^{\mathrm{A}}-H^{3^{\prime}, 5^{\prime}}, \mathrm{tpy}^{\mathrm{C}}-H^{3^{\prime}, 5^{\prime}}\right.$, $\left.\operatorname{tpy}^{\mathrm{B}}-H^{3^{\prime}, 5^{\prime}}\right), 8.77-8.71\left(\mathrm{~m}, 8 \mathrm{H}\right.$, tpy $^{\mathrm{D}}-H^{6,6^{\prime \prime}}$, tpy $^{\mathrm{C}}-H^{6,6^{\prime \prime}}$, tpy $^{\mathrm{A}}-H^{6,6^{\prime \prime}}$, tpy $\left.^{\mathrm{B}}-H^{6,6 "}\right), 8.73-8.66(\mathrm{~m}$, $8 \mathrm{H}$, tpy $^{\mathrm{D}}-H^{3,3 "}$, tpy $^{\mathrm{C}}-H^{3,3^{\prime \prime}}$, tpy $^{\mathrm{A}}-H^{3,3^{\prime \prime}}$, tpy $\left.^{\mathrm{B}}-H^{3,3 "}\right), 8.12-8.07\left(\mathrm{~m}, 2 \mathrm{H}, \mathrm{Ph}-H^{\mathrm{o}}, \mathrm{Ph}-H^{\mathrm{p}}\right), 7.98-$ $7.95\left(\mathrm{~m}, 2 \mathrm{H}, \mathrm{Ph}-H^{\mathrm{m}}\right), 7.92\left(\mathrm{~d}, J=8.3 \mathrm{~Hz}, 2 \mathrm{H}, \mathrm{Ph}-H^{\mathrm{r}}\right), 7.90-7.84\left(\mathrm{~m}, 8 \mathrm{H}, \mathrm{tpy}^{\mathrm{C}}-H^{4,4^{\prime \prime}}\right.$, $\operatorname{tpy}^{\mathrm{A}}-H^{4,4^{4}}$, tpy $^{\mathrm{B}}-H^{4,4^{4}}$, tpy $\left.^{\mathrm{D}}-H^{4,4 "}\right), 7.81\left(\mathrm{~s}, 1 \mathrm{H}, \mathrm{Ph}-H^{\mathrm{h}}\right), 7.76-7.73\left(\mathrm{~m}, 2 \mathrm{H}, \mathrm{Ph}-H^{\mathrm{n}}\right), 7.71(\mathrm{p}, J=$ $\left.1.6 \mathrm{~Hz}, 2 \mathrm{H}, \mathrm{Ph}-H^{\mathrm{j}}, \mathrm{Ph}-H^{\mathrm{i}}\right), 7.69-7.66\left(\mathrm{~m}, 2 \mathrm{H}, \mathrm{Ph}-H^{\mathrm{q}}\right), 7.58\left(\mathrm{dd}, J=8.5,2.2 \mathrm{~Hz}, 1 \mathrm{H}, \mathrm{Ph}-H^{\mathrm{b}}\right)$, $7.38-7.35\left(\mathrm{~m}, 6 \mathrm{H}, \operatorname{tpy}^{\mathrm{C}}-H^{5,5^{\prime \prime}}, \operatorname{tpy}^{\mathrm{A}}-H^{5,5^{\prime \prime}}\right.$, tpy $\left.^{\mathrm{D}}-H^{5,5^{\prime \prime}}\right), 7.32(\mathrm{ddd}, J=7.6,4.7,1.2 \mathrm{~Hz}, 2 \mathrm{H}$, $\left.\operatorname{tpy}^{\mathrm{B}}-H^{5,5 "}\right), 7.09\left(\mathrm{dt}, J=7.7,2.1 \mathrm{~Hz}, 2 \mathrm{H} \mathrm{Ph}-H^{\mathrm{l}, \mathrm{g}}\right), 7.01\left(\mathrm{~d}, J=8.6 \mathrm{~Hz}, 1 \mathrm{H}, \mathrm{Ph}-H^{\mathrm{a}}\right), 4.50(\mathrm{t}, J=$ $6.3 \mathrm{~Hz}, 2 \mathrm{H}$, Alkyl- $\left.H^{\mathrm{d}}\right), 4.06\left(\mathrm{t}, J=6.2 \mathrm{~Hz}, 2 \mathrm{H}\right.$, Alkyl $\left.-H^{\mathrm{d}}\right), 4.02(\mathrm{t}, J=6.5 \mathrm{~Hz}, 2 \mathrm{H}$, Alkyl - 
$\left.H^{\mathrm{d}^{\prime}}\right), 2.00(\mathrm{p}, J=6.5 \mathrm{~Hz}, 2 \mathrm{H}$, Alkyl-H), 1.82 (p, $J=6.8 \mathrm{~Hz}, 2 \mathrm{H}$, Alkyl- $H), 1.75$ (d, $J=9.5 \mathrm{~Hz}$, 4H, Alkyl-H), 1.49 (q, $J=7.8$ Hz, 2H, Alkyl- $H$ ), $1.44-1.48$ (m, 4H, Alkyl- $H$ ), $1.39-1.43$ (m, 4H, Alkyl-H), 1.19 (q, J = 7.5, 7.1 Hz, 2H, Alkyl-H), 1.16 - 1.09 (m, 2H, Alkyl-H), 0.97 $(\mathrm{d}, J=6.6 \mathrm{~Hz}, 2 \mathrm{H}$, Alkyl-H), $0.93-0.90$ (m, 3H, Alkyl- $H$ ), 0.88 (q, $J=8.9,7.9 \mathrm{~Hz}, 3 \mathrm{H}$, Alkyl- $H), 0.74(\mathrm{t}, J=7.2 \mathrm{~Hz}, 3 \mathrm{H}$, Alkyl- $H) .{ }^{13} \mathrm{C} \mathrm{NMR}\left(150 \mathrm{MHz}, \mathrm{CDCl}_{3}\right) \delta 162.0,159.0$, $156.8,156.5,156.2,156.1,156.1,156.1,155.3$, 149.3, 149.3, 149.2, 149.2, 148.2, 147.6, $138.4,138.3,137.0,136.8,134.4,134.1,133.9,133.6,132.6,132.3,132.3,128.6,127.5$, $127.4,127.3,124.6,124.5,124.2,124.1,124.0,124.0,123.9,123.7,121.9,121.7,121.5$, $121.5,121.3,118.7,118.7,118.5,118.3,118.2,118.1,118.0,115.1,112.3,94.1,92.8,90.8$, $90.2,89.9,89.6,88.4,87.2,87.0,86.7,75,0,68.8,68.4,53.5,32.0,31.7,31.6,30.8,29.3$, $29.2,26.3,25.9,25.8,22.9,22.7,22.5,14.3,14.2,14.1$. Maldi-TOF $(\mathrm{m} / \mathrm{z})$ : Calcd. for $\left[\mathrm{C}_{124} \mathrm{H}_{98} \mathrm{~N}_{12} \mathrm{O}_{3}+\mathrm{H}\right]^{+}:$1803.79. Found: 1803.77 .

Complexes A. To a solution of ligand $\mathbf{L A}(4.0 \mathrm{mg}, 2.2 \mu \mathrm{mol})$ in $\mathrm{CHCl}_{3}(1.5 \mathrm{~mL})$, a solution of $\mathrm{Zn}\left(\mathrm{NO}_{3}\right)_{2} \bullet 6 \mathrm{H}_{2} \mathrm{O}(1.32 \mathrm{mg}, 4.43 \mu \mathrm{mol})$ in $\mathrm{MeOH}(3.0 \mathrm{~mL})$ was added; then the mixture was stirred at $50{ }^{\circ} \mathrm{C}$ for $8 \mathrm{~h}$. After cooling to room temperature, $150 \mathrm{mg} \mathrm{NH}_{4} \mathrm{PF}_{6}$ was added and observed white precipitate, and used water to wash and obtained product $(4.8 \mathrm{mg}, 91 \%) .{ }^{1} \mathrm{H}$ NMR $\left(600 \mathrm{MHz}, \mathrm{CD}_{3} \mathrm{CN}\right) \delta 9.05-9.01\left(\mathrm{~m}, 8 \mathrm{H}\right.$, tpy $^{\mathrm{A}}-H^{3^{\prime}, 5^{\prime}}$, tpy $^{\mathrm{D}}-H^{3^{\prime}, 5^{\prime}}, \mathrm{tpy}^{\mathrm{C}}-H^{3^{\prime}, 5^{\prime}}$ and $\left.\operatorname{tpy}^{\mathrm{B}}-H^{3^{\prime}, 5^{\prime}}\right)$, 8.79-8.68(m, 6H, tpy ${ }^{\mathrm{D}}-H^{3,3^{\prime \prime}}, \operatorname{tpy}^{\mathrm{B}}-H^{3,3^{\prime \prime}}$ and tpy $\left.{ }^{\mathrm{A}}-H^{3,3 "}\right), 8.62\left(\mathrm{~m}, 2 \mathrm{H}, \operatorname{tpy}^{\mathrm{C}}-H^{3,3 "}\right), 8.48,(\mathrm{~s}, 1 \mathrm{H}$, $\left.\mathrm{Ph}-H^{\mathrm{o}}\right) \quad 8.42\left(\mathrm{~s}, 1 \mathrm{H}, \mathrm{Ph}-H^{\mathrm{p}}\right), 8.30,8.30-8.28\left(\mathrm{~m}, 4 \mathrm{H}, \mathrm{Ph}-H^{\mathrm{m}}\right), 8.13-8.10\left(\mathrm{~m}, 9 \mathrm{H}, \mathrm{tpy}^{\mathrm{A}}-H^{4,4^{\prime \prime}}\right.$, $\operatorname{tpy}^{\mathrm{B}}-H^{4,4^{\prime \prime}}, \operatorname{tpy}^{\mathrm{C}}-H^{4,4^{\prime \prime}}, \operatorname{tpy}^{\mathrm{D}}-H^{4,4^{\prime \prime}}$ and $\left.\mathrm{Ph}-H^{\mathrm{c}}\right), 8.03\left(\mathrm{~m}, 2 \mathrm{H}, \mathrm{Ph}-H^{\mathrm{n}}\right), 7.96\left(\mathrm{~m}, 2 \mathrm{H}, \mathrm{Ph}-H^{\mathrm{r}}\right)$, 7.92-7.83(m, $15 \mathrm{H}, \mathrm{Ph}-H^{\mathrm{b}}, \mathrm{Ph}-H^{\mathrm{k}}, \mathrm{Ph}-H^{\mathrm{i}}, \mathrm{Ph}-H^{\mathrm{h}}, \mathrm{Ph}-H^{\mathrm{j}}$, tpy $^{\mathrm{A}}-H^{6,6^{\prime \prime}}, \operatorname{tpy}^{\mathrm{D}}-H^{6,6 "}$, tpy $^{\mathrm{C}}-H^{6,6^{\prime \prime}}$ and tpy $\left.^{\mathrm{B}}-H^{6,6^{\prime \prime}}\right), 7.49\left(\mathrm{~m}, 1 \mathrm{H}, \mathrm{Ph}-H^{\mathrm{a}}\right), 7.39-7.21\left(\mathrm{~m}, 10 \mathrm{H}, \mathrm{Ph}-H^{1}, \mathrm{Ph}-H^{\mathrm{g}}, \operatorname{tpy}^{\mathrm{A}}-H^{5,5^{\prime \prime}}, \operatorname{tpy}^{\mathrm{D}}-H^{5,5^{\prime \prime}}\right.$ and $\left.\operatorname{tpy}^{\mathrm{C}}-H^{5,5 "}\right), 4.73\left(\mathrm{~m}, 2 \mathrm{H}\right.$, Alkyl $\left.-H^{\mathrm{d}^{\prime}}\right), 4.30\left(\mathrm{~m}, 2 \mathrm{H}, \mathrm{Alkyl}-H^{\mathrm{d}}\right), 4.14\left(\mathrm{~m}, 2 \mathrm{H}\right.$, Alkyl- $\left.H^{\mathrm{d}^{\prime}}\right), 1.55(\mathrm{~m}$, 4H, Alkyl- $H$ ), 1.43-1.42(m, 4H, Alkyl- $H), 1.31-1.26(\mathrm{~m}, 6 \mathrm{H}$, Alkyl- $H), 1.04-0.97$ (m, 10H, Alkyl- $H)$, 0.89-0.84, (m, 6H, Alkyl- $H), 0.57(\mathrm{~m}, 3 \mathrm{H}$, Alkyl- $H)$. ESI-MS $(\mathrm{m} / \mathrm{z})$ : calcd. for $\left[\mathrm{M}-15 \mathrm{PF}_{6}{ }^{-}\right]^{15+} 859.2$, found 859.2 ; calcd. for $\left[\mathrm{M}-16 \mathrm{PF}_{6}{ }^{-}\right]^{16+} 796.4$, found 796.4 ; calcd. for $\left[\mathrm{M}-17 \mathrm{PF}_{6}{ }^{-}\right]^{17+} 741.1$, found 741.1 ; calcd. for $\left[\mathrm{M}-18 \mathrm{PF}_{6}{ }^{-}\right]^{18+} 691.8$, found 691.8 ; calcd. for $\left[\mathrm{M}-19 \mathrm{PF}_{6}{ }^{-}\right]^{19+} 647.8$, found 647.8 ; calcd. for $\left[\mathrm{M}-20 \mathrm{PF}_{6}{ }^{-}\right]^{20+} 608.1$, found 608.1 ; calcd. for $\left[\mathrm{M}-21 \mathrm{PF}_{6}{ }^{-}\right]^{21+} 572.3$, found 572.3 ; calcd. for $\left[\mathrm{M}-22 \mathrm{PF}_{6}{ }^{-}\right]^{22+} 509.9$, found 509.9. 


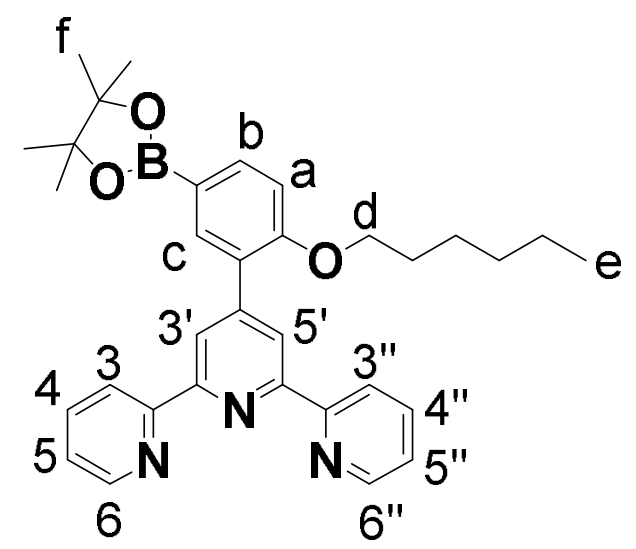

Compound 19. Compound 2 (7.37 g, $15.22 \mathrm{mmol})$, bis(pinacolato)diboron (3.83 g, 15.07 mmol $), \operatorname{Pd}\left(\mathrm{PPh}_{3}\right)_{2} \mathrm{Cl}_{2}(0.53 \mathrm{~g}, 0.76 \mathrm{mmol})$ and $\mathrm{KOAc}(8.96 \mathrm{~g}, 91.33 \mathrm{mmol})$ were mixed in a $200 \mathrm{~mL}$ Schlenk flask. After degassing and backfill with nitrogen for three times, $80 \mathrm{~mL}$ DMSO was added under nitrogen atmosphere. The mixture was stirred at $85{ }^{\circ} \mathrm{C}$ for $12 \mathrm{~h}$. After cooling down to room temperature, $100 \mathrm{~mL}$ of water was added. The mixture was extracted with $\mathrm{CH}_{2} \mathrm{Cl}_{2}$, and the organic layer was dried over anhydrous $\mathrm{Na}_{2} \mathrm{SO}_{4}$. After removal of solvent under vacuum, the residue was purified by column chromatography on silica gel with $\mathrm{CH}_{2} \mathrm{Cl}_{2}$ : methanol (100:0.5) as eluent to afford a white solid $(6.5 \mathrm{~g}, 80 \%) .{ }^{1} \mathrm{H}$ NMR (600 MHz, $\left.\mathrm{CDCl}_{3}\right) \delta 8.71-8.69\left(\mathrm{~m}, 2 \mathrm{H}\right.$, tpy- $\left.H^{6,6 "}\right), 8.67\left(\mathrm{~m}, 4 \mathrm{H}\right.$, tpy- $H^{3^{\prime}, 5^{\prime}}$ and tpy- $\left.H^{3,3 "}\right), 7.97$ (d, $\left.J=1.6 \mathrm{~Hz}, 1 \mathrm{H}, \mathrm{Ph}-H^{\mathrm{c}}\right), 7.86$ (td, $\left.J=7.7,1.8 \mathrm{~Hz}, 2 \mathrm{H}, \mathrm{tpy}-H^{4,4 "}\right), 7.82(\mathrm{dd}, J$ $\left.=8.3,1.7 \mathrm{~Hz}, 1 \mathrm{H}, \mathrm{Ph}-H^{\mathrm{b}}\right), 7.32\left(\mathrm{ddd}, J=7.4,4.7,1.2 \mathrm{~Hz}, 2 \mathrm{H}, \operatorname{tpy}-H^{5,5 "}\right), 6.98(\mathrm{~d}, J=8.3 \mathrm{~Hz}$, $\left.1 \mathrm{H}, \mathrm{Ph}-H^{\mathrm{a}}\right), 4.04\left(\mathrm{t}, J=6.3 \mathrm{~Hz}, 2 \mathrm{H}\right.$, Alkyl- $\left.H^{\mathrm{d}}\right), 1.73-1.67(\mathrm{~m}, 2 \mathrm{H}$, Alkyl- $H), 1.39$ (m, 2H, Alkyl-H), $1.35\left(\mathrm{~s}, 12 \mathrm{H}, H^{\mathrm{f}}\right), 1.18-1.14(\mathrm{~m}, 2 \mathrm{H}$, Alkyl-H), $1.12-1.07$ (m, 2H, Alkyl- $H)$, $0.72\left(\mathrm{t}, J=7.2 \mathrm{~Hz}, 3 \mathrm{H}, \mathrm{Alkyl}-H^{\mathrm{e}}\right) .{ }^{13} \mathrm{C} \mathrm{NMR}\left(150 \mathrm{MHz}, \mathrm{CDCl}_{3}\right) \delta 158.9,156.6,155.0,149.1$, $148.6,137.3,137.2,136.8,127.9,123.6,122.1,121.3,111.3,83.7,68.3,31.6,29.1,25.8$, 24.9, 24.8, 24.6, 22.4, 14.0. ESI-MS (m/z): Calcd. for $\left[\mathrm{C}_{33} \mathrm{H}_{38} \mathrm{BN}_{3} \mathrm{O}_{3}+\mathrm{H}\right]^{+}:$536.30. Found: 536.23. 


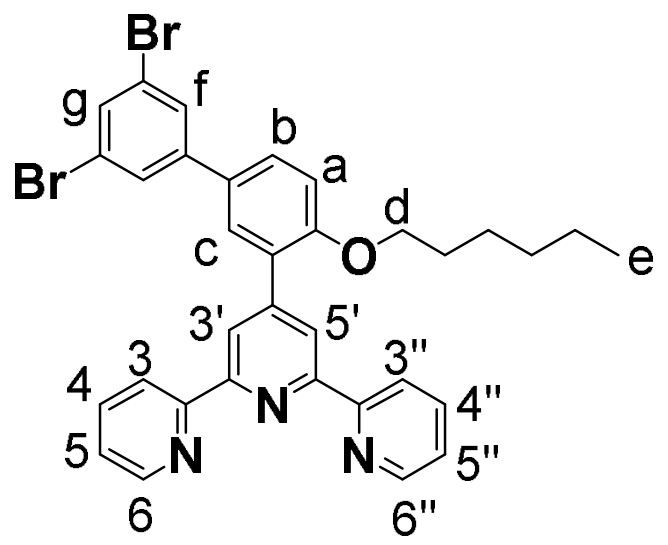

Compound 20. Compound 19 (3.0 g, $5.6 \mathrm{mmol})$, 1,3,5-tribromobenzene (5.24 g, $16.8 \mathrm{mmol}$ ), $\mathrm{Pd}\left(\mathrm{PPh}_{3}\right)_{2} \mathrm{Cl}_{2}(0.196 \mathrm{~g}, 0.28 \mathrm{mmol})$ and $\mathrm{Na}_{2} \mathrm{CO}_{3}(1.814 \mathrm{~g}, 16.8 \mathrm{mmol})$ were mixed in a 200 $\mathrm{mL}$ Schlenk flask. After degassing and backfill with nitrogen for three times, $50 \mathrm{~mL}$ toluene, $25 \mathrm{~mL} \mathrm{H}_{2} \mathrm{O}$ and $4 \mathrm{~mL}$ tert-butyl alcohol were added under nitrogen atmosphere. The mixture was stirred at $75^{\circ} \mathrm{C}$ for $12 \mathrm{~h}$. After cooling down to room temperature, $100 \mathrm{~mL}$ of water was added. The mixture was extracted with $\mathrm{CH}_{2} \mathrm{Cl}_{2}$, and the organic layer was dried over anhydrous $\mathrm{Na}_{2} \mathrm{SO}_{4}$. After removal of solvent under vacuum, the residue was purified by column chromatography on silica gel with $\mathrm{CH}_{2} \mathrm{Cl}_{2}$ : methanol (100:0.5) as eluent to afford a yellow solid (6.5 g, 80\%). ${ }^{1} \mathrm{H} \mathrm{NMR}\left(600 \mathrm{MHz}, \mathrm{CDCl}_{3}\right) \delta 8.70\left(\mathrm{~m}, 6 \mathrm{H}\right.$, tpy- $H^{6,6 "}$, tpy- $H^{3^{\prime}, 5^{\prime}}$ and tpy- $\left.H^{3,3 "}\right), 7.89$ (t, $\left.J=7.7 \mathrm{~Hz}, 2 \mathrm{H}, \operatorname{tpy}-H^{4,4 "}\right), 7.69\left(\mathrm{dd}, J=9.2,2.1 \mathrm{~Hz}, 3 \mathrm{H}, \mathrm{Ph}-H^{\mathrm{g}}, \mathrm{Ph}-H^{\mathrm{f}}\right)$, $7.60\left(\mathrm{t}, J=1.8 \mathrm{~Hz}, 1 \mathrm{H}, \mathrm{Ph}-H^{\mathrm{c}}\right), 7.53\left(\mathrm{dd}, J=8.5,2.4 \mathrm{~Hz}, 1 \mathrm{H}, \mathrm{Ph}-H^{\mathrm{b}}\right), 7.38-7.33$ (m, 2H, tpy- $\left.H^{5,5^{\prime \prime}}\right), 7.07$ (d, $\left.J=8.6 \mathrm{~Hz}, 1 \mathrm{H}, \mathrm{Ph}-H^{\mathrm{a}}\right), 4.06\left(\mathrm{t}, J=6.3 \mathrm{~Hz}, 2 \mathrm{H}\right.$, Alkyl- $\left.H^{\mathrm{d}}\right), 1.75-1.69(\mathrm{~m}$, 2H, Alkyl-H), 1.40 (m, 2H, Alkyl-H), $1.21-1.10$ (m, 4H, Alkyl-H), 0.73 (t, $J=7.2 \mathrm{~Hz}, 3 \mathrm{H}$, Alkyl- $\left.H^{\mathrm{e}}\right) .{ }^{13} \mathrm{C}$ NMR $\left(150 \mathrm{MHz}, \mathrm{CDCl}_{3}\right) \delta 156.8,156.5,155.3,149.2,148.1,144.2,136.9$, 132.2 , 130.8, 129.2, 129.1, 128.7, 128.6, 123.8, 123.3, 121.9, 121.4, 112.7, 68.8, 31.6, 29.2, 25.9, 22.5, 14.1. ESI-MS (m/z): Calcd. for $\left[\mathrm{C}_{33} \mathrm{H}_{29} \mathrm{Br}_{2} \mathrm{~N}_{3} \mathrm{O}+\mathrm{H}\right]^{+}:$642.07. Found: 641.98 . 


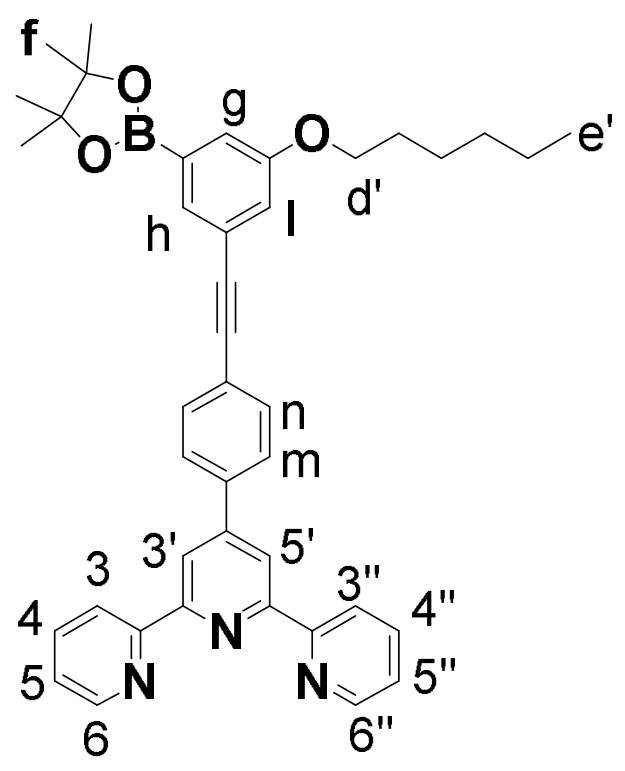

Compound 21. Compound 10 (3.26 g, $5.53 \mathrm{mmol})$, bis(pinacolato)diboron (1.378 g, 5.42 $\mathrm{mmol}), \mathrm{Pd}\left(\mathrm{PPh}_{3}\right)_{2} \mathrm{Cl}_{2}(193.7 \mathrm{mg}, 0.276 \mathrm{mmol})$ and $\mathrm{KOAc}(3.25 \mathrm{~g}, 33.2 \mathrm{mmol})$ were mixed in a $200 \mathrm{~mL}$ Schlenk flask. After degassing and backfill with nitrogen for three times, $60 \mathrm{~mL}$ DMSO was added under nitrogen atmosphere. The mixture was stirred at $85{ }^{\circ} \mathrm{C}$ for $12 \mathrm{~h}$. After cooling down to room temperature, $100 \mathrm{~mL}$ of water was added. The mixture was extracted with $\mathrm{CH}_{2} \mathrm{Cl}_{2}$, and the organic layer was dried over anhydrous $\mathrm{Na}_{2} \mathrm{SO}_{4}$. After removal of solvent under vacuum, the residue was purified by column chromatography on silica gel with $\mathrm{CH}_{2} \mathrm{Cl}_{2}$ : methanol (100:0.7) as eluent to afford a yellow solid $(2.2 \mathrm{~g}, 65 \%) .{ }^{1} \mathrm{H}$ NMR (600 MHz, $\left.\mathrm{CDCl}_{3}\right) \delta 8.76\left(\mathrm{~s}, 2 \mathrm{H}, \mathrm{tpy}-H^{3^{\prime}, 5^{\prime}}\right), 8.75-8.74\left(\mathrm{~m}, 2 \mathrm{H}, \mathrm{tpy}-H^{6,6^{\prime \prime}}\right), 8.70-8.68$ $\left(\mathrm{m}, 2 \mathrm{H}, \mathrm{tpy}-H^{3,3 "}\right), 7.93-7.89\left(\mathrm{~m}, 4 \mathrm{H}, \mathrm{Ph}-H^{\mathrm{m}}\right.$ and tpy- $\left.H^{4,4 "}\right), 7.66-7.64\left(\mathrm{~m}, 2 \mathrm{H}, \mathrm{Ph}-H^{\mathrm{n}}\right), 7.62$ (t, $\left.J=1.1 \mathrm{~Hz}, 1 \mathrm{H}, \mathrm{Ph}-H^{1}\right), 7.37\left(\mathrm{ddd}, J=7.5,4.8,1.2 \mathrm{~Hz}, 2 \mathrm{H}, \mathrm{tpy}-H^{5,5^{\prime \prime}}\right), 7.33-7.31(\mathrm{~m}, 1 \mathrm{H}$, Ph- $\left.H^{\mathrm{h}}\right), 7.18\left(\mathrm{dd}, J=2.7,1.5 \mathrm{~Hz}, 1 \mathrm{H}, \mathrm{Ph}-H^{\mathrm{g}}\right), 4.01\left(\mathrm{t}, J=6.5 \mathrm{~Hz}, 2 \mathrm{H}\right.$, Alkyl- $\left.H^{\mathrm{d}^{\mathrm{d}}}\right), 1.80-1.77$ (m, 2H, Alkyl-H), $1.49-1.45$ (m, 2H, Alkyl- $H), 1.36$ (s, 12H, Alkyl- $H), 0.94-0.90$ (m, 3H, Alkyl- $\left.H^{\mathrm{e}^{\prime}}\right) .{ }^{13} \mathrm{C} \mathrm{NMR}\left(150 \mathrm{MHz}, \mathrm{CDCl}_{3}\right) \delta 158.6,156.2,156.1,149.4,149.2,138.1,137.0$, 132.2 , 130.6, 127.3, 124.2, 124.0, 123.8, 121.4, 120.9, 120.2, 118.7, 91.0, 89.0, 84.1, 68.2, 31.7, 29.3, 29.3, 25.8, 25.0, 22.7, 14.2. ESI-MS (m/z): Calcd. for $\left[\mathrm{C}_{41} \mathrm{H}_{42} \mathrm{BN}_{3} \mathrm{O}_{3}+\mathrm{H}\right]^{+}: 636.33$. Found: 636.25. 


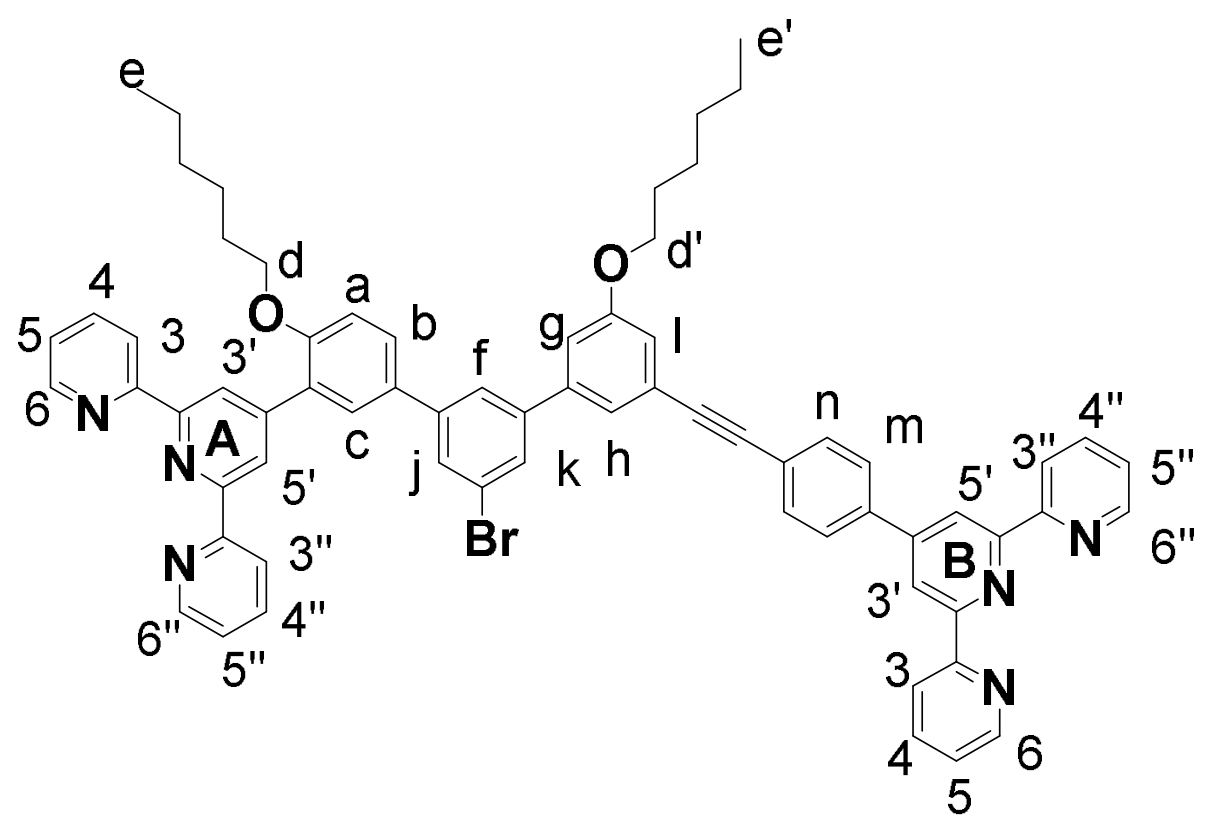

Compound 22. Compound 20 (1.65 g, $2.57 \mathrm{mmol})$, compound 21 (0.817 g, $1.28 \mathrm{mmol})$, $\mathrm{Pd}\left(\mathrm{PPh}_{3}\right)_{2} \mathrm{Cl}_{2}(45 \mathrm{mg}, 0.06 \mathrm{mmol})$ and $\mathrm{Na}_{2} \mathrm{CO}_{3}(0.42 \mathrm{~g}, 3.86 \mathrm{mmol})$ were mixed in a $200 \mathrm{~mL}$ Schlenk flask. After degassing and backfill with nitrogen for three times, $50 \mathrm{~mL}$ toluene, 25 $\mathrm{mL} \mathrm{H}_{2} \mathrm{O}$ and $5 \mathrm{~mL}$ tert-butyl alcohol were added under nitrogen atmosphere. The mixture was stirred at $75^{\circ} \mathrm{C}$ for $12 \mathrm{~h}$. After cooling down to room temperature, $100 \mathrm{~mL}$ of water was added. The mixture was extracted with $\mathrm{CH}_{2} \mathrm{Cl}_{2}$, and the organic layer was dried over anhydrous $\mathrm{Na}_{2} \mathrm{SO}_{4}$. After removal of solvent under vacuum, the residue was purified by column chromatography on silica gel with $\mathrm{CH}_{2} \mathrm{Cl}_{2}$ : methanol $(100: 1.5)$ as eluent to afford a yellow solid (0.9 g, 66\%). ${ }^{1} \mathrm{H}$ NMR $\left(600 \mathrm{MHz}, \mathrm{CDCl}_{3}\right) \delta 8.76\left(\mathrm{~s}, 2 \mathrm{H}\right.$, tpy $\left.^{\mathrm{B}}-H^{3^{\prime}, 5^{\prime}}\right), 8.75-8.73$ $\left(\mathrm{m}, 4 \mathrm{H}, \operatorname{tpy}^{\mathrm{B}}-H^{6,6^{\prime \prime}}, \operatorname{tpy}^{\mathrm{A}}-H^{3^{\prime}, 5^{\prime}}\right), 8.71-8.68\left(\mathrm{~m}, 6 \mathrm{H}, \operatorname{tpy}^{\mathrm{A}}-H^{6,6^{\prime \prime}}, \operatorname{tpy}^{\mathrm{B}}-H^{3,3^{\prime \prime}}, \operatorname{tpy}^{\mathrm{A}}-H^{3,3 "}\right), 7.93-$ $7.91\left(\mathrm{~m}, 2 \mathrm{H}, \mathrm{Ph}-H^{\mathrm{m}}\right), 7.90-7.86\left(\mathrm{~m}, 4 \mathrm{H}, \operatorname{tpy}^{\mathrm{B}}-H^{4,4^{\prime \prime}}, \operatorname{tpy}^{\mathrm{A}}-H^{4,4 "}\right), 7.79(\mathrm{~d}, J=2.4 \mathrm{~Hz}, 1 \mathrm{H}$, $\left.\mathrm{Ph}-H^{\mathrm{k}}\right), 7.75\left(\mathrm{dt}, J=7.7,1.7 \mathrm{~Hz}, 2 \mathrm{H}, \mathrm{Ph}-H^{\mathrm{j}}, \mathrm{Ph}-H^{\mathrm{f}}\right), 7.71-7.68\left(\mathrm{~m}, 3 \mathrm{H}, \mathrm{Ph}-H^{\mathrm{c}}\right.$, $\left.\mathrm{Ph}-H^{\mathrm{n}}\right), 7.63-7.62\left(\mathrm{~d}, J=8.5 \mathrm{~Hz}, 1 \mathrm{H}, \mathrm{Ph}-H^{\mathrm{b}}\right), 7.43\left(\mathrm{t}, J=1.5 \mathrm{~Hz}, 1 \mathrm{H}, \mathrm{Ph}-H^{\mathrm{l}}\right), 7.38(\mathrm{ddd}, J=7.4$, 4.7, $1.2 \mathrm{~Hz}, 2 \mathrm{H}$, tpy $\left.^{\mathrm{B}}-H^{5,5 "}\right), 7.33\left(\mathrm{ddd}, J=7.4,4.8,1.2 \mathrm{~Hz}, 2 \mathrm{H}, \mathrm{tpy}^{\mathrm{A}}-H^{5,5 "}\right), 7.15(\mathrm{t}, J=2.0 \mathrm{~Hz}$, $\left.1 \mathrm{H}, \mathrm{Ph}-H^{\mathrm{g}}\right), 7.11-7.08\left(\mathrm{~m}, 3 \mathrm{H}, \mathrm{Ph}-H^{\mathrm{a}}, \mathrm{Ph}-H^{\mathrm{h}}\right), 4.07$ (t, $J=6.3 \mathrm{~Hz}, 2 \mathrm{H}$, Alkyl- $\left.H^{\mathrm{d}}\right), 4.05(\mathrm{t}, J=$ $6.5 \mathrm{~Hz}, 2 \mathrm{H}$, Alkyl- $\left.H^{\mathrm{d}^{\prime}}\right), 1.84-1.80$ (m, 2H, Alkyl-H), $1.76-1.72$ (m, 2H, Alkyl- $\left.H\right)$, 1.50(m,2H, Alkyl-H)1.44 - 1.40 (m, 2H, Alkyl- $H), 1.36$ (m, 4H, Alkyl-H), $1.22-1.17$ (m, 2H, Alkyl-H), $1.15-1.10$ (m, 3H, Alkyl-H), 0.91 (m, 3H, Alkyl-H), 0.74 (t, J=7.2 Hz, 3H, Alkyl- $H) .{ }^{13} \mathrm{C}$ NMR $\left(150 \mathrm{MHz}, \mathrm{CDCl}_{3}\right) \delta 159.5,156.6,156.2,156.1,155.3,149.4,149.2$, 
$148.3,143.2,142.8,141.4,138.3,137.0,136.9,132.3,132.2,129.4,129.1,129.1,128.7$, $128.5,127.4,124.6,124.6,124.0,124.0,123.7,123.1,122.0,121.5,121.4,118.7,116.3$, $112.7,90.9,89.3,68.8,68.4,31.7,31.7,29.8,29.3,29.2,25.9,25.8,22.7,22.5,14.2,14.1$. ESI-MS (m/z): Calcd. for $\left[\mathrm{C}_{68} \mathrm{H}_{59} \mathrm{BrN}_{6} \mathrm{O}_{2}+\mathrm{H}\right]^{+}: 1071.39$, Found: 1071.41.

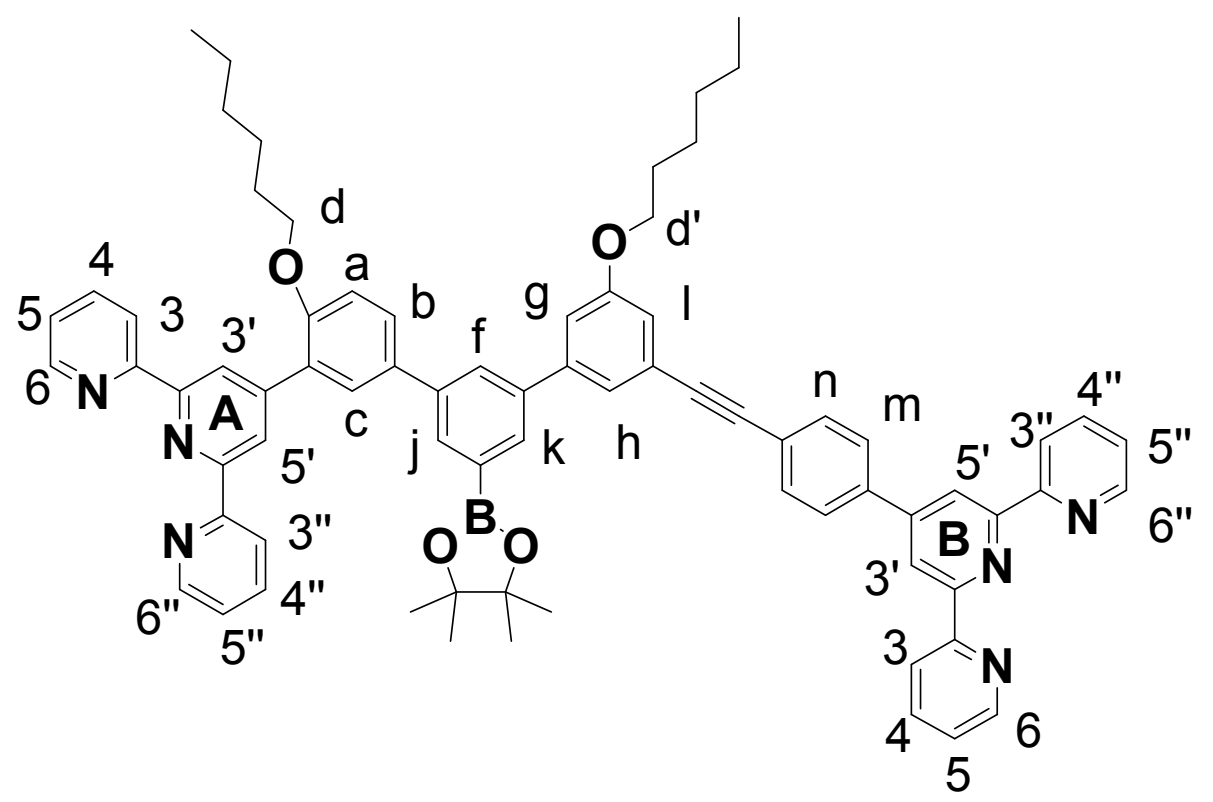

Compound 23. Compound 22 (0.916 g, $0.855 \mathrm{mmol})$, bis(pinacolato)diboron (0.2827 g, $1.112 \mathrm{mmol}), \mathrm{Pd}\left(\mathrm{PPh}_{3}\right)_{2} \mathrm{Cl}_{2}(31.3 \mathrm{mg}, 0.0427 \mathrm{mmol})$ and $\mathrm{KOAc}(0.503 \mathrm{~g}, 5.13 \mathrm{mmol})$ were mixed in a $200 \mathrm{~mL}$ Schlenk flask. After degassing and backfill with nitrogen for three times, $45 \mathrm{~mL}$ DMSO was added under nitrogen atmosphere. The mixture was stirred at $85^{\circ} \mathrm{C}$ for 24 h. After cooling down to room temperature, $100 \mathrm{~mL}$ of water was added. The mixture was extracted with $\mathrm{CH}_{2} \mathrm{Cl}_{2}$, and the organic layer was dried over anhydrous $\mathrm{Na}_{2} \mathrm{SO}_{4}$. After removal of solvent under vacuum, the residue was purified by column chromatography on silica gel with $\mathrm{CH}_{2} \mathrm{Cl}_{2}$ : methanol (100:2) as eluent to afford a white solid $(0.4 \mathrm{~g}, 41 \%){ }^{1} \mathrm{H}$ NMR (600 MHz, $\left.\mathrm{CDCl}_{3}\right) \delta 8.77\left(\mathrm{~s}, 2 \mathrm{H}\right.$, tpy $\left.^{\mathrm{B}}-H^{3^{\prime}, 5^{\prime}}\right), 8.75\left(\mathrm{~m}, 4 \mathrm{H}, \mathrm{tpy}^{\mathrm{A}}-H^{3^{\prime}, 5^{\prime}}\right.$, tpy $\left.^{\mathrm{B}}-H^{6,6^{\prime \prime}}\right), 8.69$ $\left(\mathrm{m}, 6 \mathrm{H}, \operatorname{tpy}^{\mathrm{A}}-H^{6,6^{\prime \prime}}, \operatorname{tpy}^{\mathrm{A}}-H^{3,3 "}, \operatorname{tpy}^{\mathrm{B}}-H^{3,3^{\prime \prime}}\right), 8.05\left(\mathrm{~s}, 1 \mathrm{H}, \mathrm{Ph}-H^{\mathrm{j}}\right), 8.02\left(\mathrm{~s}, 1 \mathrm{H}, \mathrm{Ph}-H^{\mathrm{k}}\right), 7.92(\mathrm{~m}$, $\left.3 \mathrm{H}, \mathrm{Ph}-H^{\mathrm{m}}, \mathrm{Ph}-H^{\mathrm{c}}\right), 7.90-7.84\left(\mathrm{~m}, 5 \mathrm{H}, \mathrm{Ph}-H^{\mathrm{f}}, \mathrm{tpy}^{\mathrm{A}}-H^{4,4^{4}}, \mathrm{tpy}^{\mathrm{B}}-H^{4,4 "}\right), 7.71-7.68\left(\mathrm{~m}, 3 \mathrm{H}, \mathrm{Ph}-H^{\mathrm{b}}\right.$, $\left.\mathrm{Ph}-H^{\mathrm{n}}\right), 7.51\left(\mathrm{~s}, 1 \mathrm{H}, \mathrm{Ph}-H^{\mathrm{l}}\right), 7.37\left(\mathrm{dd}, J=7.5,4.8 \mathrm{~Hz}, 2 \mathrm{H}, \mathrm{tpy}^{\mathrm{A}}-H^{5,5}\right), 7.32(\mathrm{dd}, J=7.5,4.9$ $\left.\mathrm{Hz}, 2 \mathrm{H}, \operatorname{tpy}^{\mathrm{B}}-H^{5,5^{\prime}}\right), 7.24\left(\mathrm{~s}, 1 \mathrm{H}, \mathrm{Ph}-H^{\mathrm{g}}\right), 7.09$ (d, $\left.J=9.0 \mathrm{~Hz}, 2 \mathrm{H}, \mathrm{Ph}-H^{\mathrm{a}}, \mathrm{Ph}-H^{\mathrm{h}}\right), 4.06(\mathrm{~d}, J=$ $7.0 \mathrm{~Hz}, 4 \mathrm{H}$, Alkyl- $H^{\mathrm{d}}$, Alkyl- $\left.H^{\mathrm{d}}\right), 1.82$ (s, 2H, Alkyl- $\left.H\right), 1.72$ (s, 2H, Alkyl-H), 1.50 (s, 2H, Alkyl- $H$ ), 1.43 (s, 2H, Alkyl-H), 1.36 (s, 4H, Alkyl-H), 1.24 (s, 12H, Alkyl- $H$ ), 1.19 (s, 2H, 
Alkyl- $H$ ), 1.14 (s, 2H, Alkyl- $H$ ), 0.91 (s, 3H, Alkyl- $H$ ), 0.74 (s, 3H, Alkyl- $H) .{ }^{13} \mathrm{C}$ NMR (150 $\left.\mathrm{MHz}, \mathrm{CDCl}_{3}\right) \delta 159.7,156.2,156.1,155.2,149.2,149.2,148.6,142.9,140.7,140.3,138.2$, $137.0,136.8,133.8,132.8,132.3,132.2,132.2,132.1,132.1,131.4,129.5,128.9,128.7$, $128.6,128.6,127.3,124.3,124.2,124.0,123.6,123.4,122.2,121.5,121.3,118.7,115.7$, $115.2,112.7,91.2,89.0,84.1,82.9,75.1,68.8,68.4,31.7,31.7,29.4,29.3,25.9,25.8,25.0$, 24.9, 24.7, 22.7, 22.5, 14.2, 14.1. ESI-MS (m/z): Calcd. for $\left[\mathrm{C}_{74} \mathrm{H}_{71} \mathrm{BN}_{6} \mathrm{O}_{4}+2 \mathrm{H}\right]^{2+}: 560.28$. Found: 560.18 .

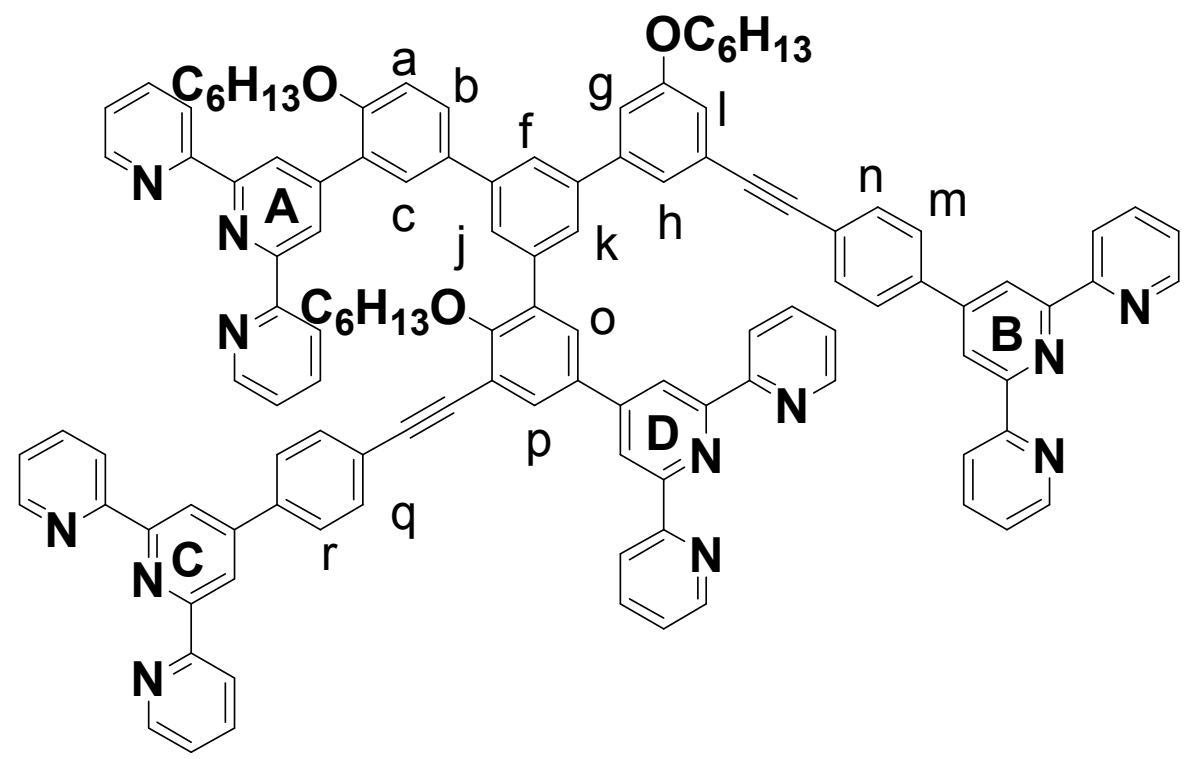

Ligand LB. Compound 23 (0.3519 g, 0.314 mmol), compound 16 (0.3868 g, 0.471 mmol), $\mathrm{Pd}\left(\mathrm{PPh}_{3}\right)_{2} \mathrm{Cl}_{2}(11 \mathrm{mg}, 0.0157 \mathrm{mmol})$ and $\mathrm{Na}_{2} \mathrm{CO}_{3}(0.1 \mathrm{~g}, 0.943 \mathrm{mmol})$ were mixed in a 100 $\mathrm{mL}$ Schlenk flask. After degassing and backfill with nitrogen for three times, $30 \mathrm{~mL}$ toluene, $15 \mathrm{~mL} \mathrm{H}_{2} \mathrm{O}$ and $5 \mathrm{~mL}$ tert-butyl alcohol were added under nitrogen atmosphere. The mixture was stirred at $75^{\circ} \mathrm{C}$ for $24 \mathrm{~h}$. After cooling down to room temperature, $100 \mathrm{~mL}$ of water was added. The mixture was extracted with $\mathrm{CHCl}_{3}$, and the organic layer was dried over anhydrous $\mathrm{Na}_{2} \mathrm{SO}_{4}$. After removal of solvent under vacuum, the residue was purified by column chromatography on silica gel with $\mathrm{CH}_{2} \mathrm{Cl}_{2}$ : methanol $(100: 1.5)$ as eluent to afford a yellow solid (0.217g, 40\%). ${ }^{1} \mathrm{H}$ NMR $\left(600 \mathrm{MHz}, \mathrm{CDCl}_{3}\right) \delta 8.80\left(\mathrm{~s}, 2 \mathrm{H}, \mathrm{tpy}^{\mathrm{C}}-H^{3^{\prime}, 5^{\prime}}\right), 8.78(\mathrm{~s}$, $\left.2 \mathrm{H}, \operatorname{tpy}^{\mathrm{B}}-H^{3^{\prime}, 5^{\prime}}\right), 8.77\left(\mathrm{~s}, 2 \mathrm{H}, \operatorname{tpy}^{\mathrm{A}}-H^{3^{\prime}, 5^{\prime}}\right), 8.75\left(\mathrm{~s}, 2 \mathrm{H}, \operatorname{tpy}^{\mathrm{D}}-H^{3^{\prime}, 5^{\prime}}\right), 8.73\left(\mathrm{~m}, 6 \mathrm{H}, \operatorname{tpy}^{\mathrm{C}}-H^{6,6^{\prime \prime}}\right.$, $\left.\operatorname{tpy}^{\mathrm{B}}-H^{6,6^{\prime \prime}}, \operatorname{tpy}^{\mathrm{A}}-H^{6,6 "}\right), 8.70-8.65\left(\mathrm{~m}, 10 \mathrm{H}, \operatorname{tpy}^{\mathrm{D}}-H^{6,6^{\prime \prime}}, \operatorname{tpy}^{\mathrm{C}}-H^{3,3^{\prime \prime}}, \operatorname{tpy}^{\mathrm{A}}-H^{3,3^{\prime \prime}}, \operatorname{tpy}^{\mathrm{B}}-H^{3,3^{\prime \prime}}\right.$, $\left.\operatorname{tpy}^{\mathrm{D}}-H^{3,3 "}\right), 8.18\left(\mathrm{~d}, J=2.3 \mathrm{~Hz}, 1 \mathrm{H}, \mathrm{Ph}-H^{\mathrm{p}}\right), 8.02\left(\mathrm{~d}, J=2.4 \mathrm{~Hz}, 1 \mathrm{H}, \mathrm{Ph}-H^{\mathrm{o}}\right), 7.96(\mathrm{~d}, J=8.0$ $\left.\mathrm{Hz}, 2 \mathrm{H}, \mathrm{Ph}-H^{\mathrm{m}}\right), 7.93$ (d, $\left.J=2.0 \mathrm{~Hz}, 2 \mathrm{H}, \mathrm{Ph}-H^{\mathrm{q}}\right), 7.88$ (m, 12H, Ph- $H^{\mathrm{k}}, \mathrm{Ph}-H^{\mathrm{l}}, \mathrm{Ph}-H^{\mathrm{c}}, \mathrm{Ph}-H^{\mathrm{f}}$, 
$\operatorname{tpy}^{\mathrm{A}}-H^{4,4^{\prime \prime}}$, tpy $^{\mathrm{B}}-H^{4,4^{\prime \prime}}$, tpy $^{\mathrm{C}}-H^{4,4^{\prime \prime}}$, tpy $\left.^{\mathrm{D}}-H^{4,4 "}\right), 7.77\left(\mathrm{~m}, 3 \mathrm{H}, \mathrm{Ph}-H^{\mathrm{n}}, \mathrm{Ph}-H^{\mathrm{b}}\right), 7.69(\mathrm{~d}, J=8.1 \mathrm{~Hz}$, $\left.2 \mathrm{H}, \mathrm{Ph}-H^{\mathrm{r}}\right), 7.55\left(\mathrm{~s}, 1 \mathrm{H}, \mathrm{Ph}-H^{\mathrm{j}}\right), 7.35\left(\mathrm{~m}, 6 \mathrm{H}, \operatorname{tpy}^{\mathrm{B}}-H^{5,5^{\prime \prime}}, \operatorname{tpy}^{\mathrm{C}}-H^{5,5^{\prime \prime}}, \operatorname{tpy}^{\mathrm{D}}-H^{5,5^{\prime \prime}}\right), 7.29(\mathrm{~m}, 3 \mathrm{H}$ $\left.\operatorname{tpy}^{\mathrm{A}}-H^{5,5^{\prime \prime}}, \mathrm{Ph}-H^{\mathrm{h}}\right), 7.14-7.10\left(\mathrm{~m}, 2 \mathrm{H}, \mathrm{Ph}-H^{\mathrm{a}}, \mathrm{Ph}-H^{\mathrm{g}}\right), 4.08(\mathrm{dt}, J=10.4,6.3 \mathrm{~Hz}, 4 \mathrm{H}$, Alkyl- $H$ ), 4.01 (t, $J=6.2 \mathrm{~Hz}, 2 \mathrm{H}$, Alkyl- $H), 1.85-1.80$ (m, 4H, Alkyl- $H$ ), 1.75 (dd, $J=8.9$, $6.2 \mathrm{~Hz}, 2 \mathrm{H}$, Alkyl- $H$ ), 1.68 (m, 2H, Alkyl- $H), 1.50$ (t, $J=7.6 \mathrm{~Hz}, 2 \mathrm{H}$, Alkyl- $H$ ), 1.43 (m, 2H, Alkyl-H), 1.35 (m, 6H, Alkyl-H), $1.23-1.18$ (m, 4H, Alkyl-H), $1.17-1.11$ (m, 6H, Alkyl-H), 0.90 (t, $J=6.9 \mathrm{~Hz}, 3 \mathrm{H}$, Alkyl- $H), 0.75$ (q, $J=6.7 \mathrm{~Hz}, 6 \mathrm{H}$, Alkyl- $H) .{ }^{13} \mathrm{C}$ NMR $(150 \mathrm{MHz}$, $\left.\mathrm{CDCl}_{3}\right) \delta 159.6,158.8,156.6,156.3,156.2,156.2,156.1,156.1,155.3,149.4,149.2,149.2$, $149.2,149.0,148.5,148.4,142.7,141.3,140.9,138.9,138.3,138.1,136.9,136.9,136.8$, $136.3,134.4,133.5,132.4,132.3,129.5,129.1,128.9,127.4,127.4,127.3,126.9,124.5$, 124.2 , 124.2, 124.0, 123.6, 123.4, 122.1, 121.4, 121.3, 118.9, 118.8, 118.7, 118.7, 115.0, $112.8,93.7,91.2,89.1,87.7,74.7,68.9,68.4,31.8,31.7,31.7,30.5,29.8,29.4,29.3,26.0$, 25.9, 25.9, 22.7, 22.6, 22.5, 14.2, 14.2, 14.1. MALDI-TOF $(\mathrm{m} / \mathrm{z})$ : Calcd. for $\left[\mathrm{C}_{118} \mathrm{H}_{98} \mathrm{~N}_{12} \mathrm{O}_{3}+\mathrm{H}\right]^{+}:$1731.79. Found: 1731.75.

Complexes B. To a solution of ligand $\mathbf{L B}(5.4 \mathrm{mg}, 3.11 \mu \mathrm{mol})$ in $\mathrm{CHCl}_{3}(2 \mathrm{~mL})$, a solution of $\mathrm{Zn}\left(\mathrm{NO}_{3}\right)_{2} \cdot 6 \mathrm{H}_{2} \mathrm{O}(1.856 \mathrm{mg}, 6.23 \mu \mathrm{mol})$ in $\mathrm{MeOH}(4 \mathrm{~mL})$ was added; then the mixture was

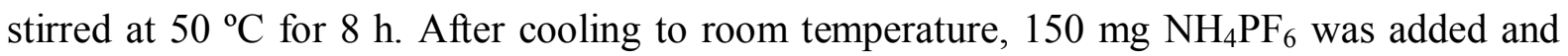
observed white precipitate, and used water to wash and obtained product $(6.8 \mathrm{mg}, 95 \%)$. ${ }^{1} \mathrm{H}$ NMR $\left(600 \mathrm{MHz}, \mathrm{CD}_{3} \mathrm{CN}\right) \delta 9.17\left(\mathrm{~s}, 4 \mathrm{H}, \operatorname{tpy}^{\mathrm{C}}-H^{3^{\prime}, 5^{\prime}}, \operatorname{tpy}^{\mathrm{B}}-H^{3^{\prime}, 5^{\prime}}\right), 9.01\left(\mathrm{~s}, 4 \mathrm{H}, \operatorname{tpy}^{\mathrm{A}}-H^{3^{\prime}, 5^{\prime}}\right.$, $\left.\operatorname{tpy}^{\mathrm{D}}-H^{3^{\prime}, 5^{\prime}}\right), 8.66\left(\mathrm{~m}, 10 \mathrm{H}, \operatorname{tpy}^{\mathrm{A}}-H^{3,3^{\prime \prime}}, \operatorname{tpy}^{\mathrm{B}}-H^{3,3^{\prime \prime}}, \operatorname{tpy}^{\mathrm{C}}-H^{3,3 "}, \operatorname{tpy}^{\mathrm{D}}-H^{3,3 "}\right), 8.34-8.30(\mathrm{~m}, 8 \mathrm{H}$ tpy $^{\mathrm{C}}-H^{6,6^{\prime \prime}}$, tpy $\left.^{\mathrm{B}}-H^{6,6^{\prime \prime}}, \mathrm{Ph}-H^{\mathrm{q}}, \mathrm{Ph}-H^{\mathrm{m}}\right), 8.12\left(\mathrm{~s}, 2 \mathrm{H}, \mathrm{Ph}-H^{\mathrm{r}}\right) 7.78\left(\mathrm{~m}, 14 \mathrm{H}\right.$, tpy $^{\mathrm{A}}-H^{6,6^{\prime \prime}}$, tpy $^{\mathrm{D}}-H^{6,6^{\prime \prime}}$, $\operatorname{tpy}^{\mathrm{A}}-H^{4,4^{\prime \prime}}$, tpy $^{\mathrm{B}}-H^{4,4^{\prime \prime}}$, tpy $^{\mathrm{C}}-H^{4,4 "}$, tpy $\left.^{\mathrm{D}}-H^{4,4 "}\right), 7.58-7.49\left(\mathrm{~m}, 3 \mathrm{H}, \mathrm{Ph}-H^{\mathrm{f}}, \mathrm{Ph}-H^{\mathrm{k}}, \mathrm{Ph}-H^{\mathrm{l}}\right), 7.30(\mathrm{~s}$, $\left.1 \mathrm{H}, \mathrm{Ph}-H^{\mathrm{h}}\right), 7.17-6.88\left(\mathrm{~m}, 10 \mathrm{H}, \mathrm{Ph}-H^{\mathrm{a}}, \mathrm{Ph}-H^{\mathrm{g}}, \operatorname{tpy}^{\mathrm{A}}-H^{5,5^{\prime \prime}}, \operatorname{tpy}^{\mathrm{B}}-H^{5,5^{\prime \prime}}, \operatorname{tpy}^{\mathrm{C}}-H^{5,5^{\prime \prime}}, \operatorname{tpy}^{\mathrm{D}}-H^{5,5^{\prime \prime}}\right)$, 4.45-4.15 (m, 6H), $1.57(\mathrm{~s}, 6 \mathrm{H}), 1.44(\mathrm{~s}, 6 \mathrm{H}), 1.21(\mathrm{~s}, 12 \mathrm{H}), 1.00(\mathrm{~s}, 3 \mathrm{H}), 0.85(\mathrm{~s}, 3 \mathrm{H}), 0.70$ (s,3H). ESI-MS $(\mathrm{m} / \mathrm{z})$ : B1 [ $\left.\mathbf{Z n}_{12} \mathbf{L B}_{6}\right]$ : calcd. for [M-12PF$\left.{ }_{6}^{-}\right]^{12+}$ 1074.2, found 1074.2; calcd. for $\left[\mathrm{M}-13 \mathrm{PF}_{6}{ }^{-}\right]^{13+} 980.5$, found 980.5 ; calcd. for $\left[\mathrm{M}-14 \mathrm{PF}_{6}{ }^{-}\right]^{14+} 900.1$, found 900.1 ; calcd. for $\left[\mathrm{M}-15 \mathrm{PF}_{6}^{-}\right]^{15+} 830.4$, found 830.4 ; calcd. for $\left[\mathrm{M}-16 \mathrm{PF}_{6}{ }^{-}\right]^{16+} 769.4$, found 769.4. B2 $\left[\mathbf{Z n}_{14} \mathbf{L B}_{7}\right]$ : calcd. for $\left[\mathrm{M}-12 \mathrm{PF}_{6}{ }^{-}\right]^{12+} 1277.5$, found 1277.5 ; calcd. for $\left[\mathrm{M}-13 \mathrm{PF}_{6}{ }^{-}\right]^{13+} 1168.0$, found 1168.0; calcd. for $\left[\mathrm{M}-14 \mathrm{PF}_{6}{ }^{-}\right]^{14+} 1074.2$, found 1074.2 ; calcd. for $\left[\mathrm{M}-15 \mathrm{PF}_{6}{ }^{-}\right]^{15+} 993.0$, 
found 993.0; calcd. for $\left[\mathrm{M}-16 \mathrm{PF}_{6}{ }^{-}\right]^{16+} 921.8$, found 921.8; calcd. for $\left[\mathrm{M}-17 \mathrm{PF}_{6}{ }^{-}\right]^{17+} 859.1$, found 859.1; calcd. for $\left[\mathrm{M}-18 \mathrm{PF}_{6}{ }^{-}\right]^{18+} 803.3$, found 803.3; calcd. for $\left[\mathrm{M}-19 \mathrm{PF}_{6}{ }^{-}\right]^{19+} 753.4$, found 753.4; calcd. for $\left[\mathrm{M}-20 \mathrm{PF}_{6}{ }^{-}\right]^{20+} 708.5$, found 708.5 ; calcd. for $\left[\mathrm{M}-21 \mathrm{PF}_{6}{ }^{-}\right]^{21+} 667.8$, found 667.8. $\mathbf{B 3}\left[\mathbf{Z n}_{16} \mathbf{L B}_{8}\right]$ : calcd. for $\left[\mathrm{M}-12 \mathrm{PF}_{6}{ }^{-}\right]^{12+} 1480.7$, found 1480.7; calcd. for $\left[\mathrm{M}-13 \mathrm{PF}_{6}{ }^{-}\right]^{13+} 1355.6$, found 1355.6 ; calcd. for $\left[\mathrm{M}-14 \mathrm{PF}_{6}{ }^{-14+}\right]^{14}$ 1248.4, found 1248.4; calcd. for $\left[\mathrm{M}-15 \mathrm{PF}_{6}{ }^{-}\right]^{15+} 1155.5$, found 1155.5 ; calcd. for $\left[\mathrm{M}-16 \mathrm{PF}_{6}{ }^{-}\right]^{16+} 1074.2$, found 1074.2; calcd. for $\left[\mathrm{M}-17 \mathrm{PF}_{6}{ }^{-}\right]^{17+} 1002.5$, found 1002.5 ; calcd. for $\left[\mathrm{M}-18 \mathrm{PF}_{6}{ }^{-}\right]^{18+} 938.8$, found 938.8 ; calcd. for $\left[\mathrm{M}-19 \mathrm{PF}_{6}{ }^{-}\right]^{19+} 881.7$, found 881.7 ; calcd. for $\left[\mathrm{M}-20 \mathrm{PF}_{6}{ }^{-20+}\right]^{20} 830.4$, found 830.4; calcd. for $\left[\mathrm{M}-21 \mathrm{PF}_{6}{ }^{-}\right]^{21+} 783.9$, found 783.9 ; calcd. for $\left[\mathrm{M}-22 \mathrm{PF}_{6}{ }^{-}\right]^{22+} 741.7$, found 741.7 . 


\section{4. ${ }^{1} \mathrm{H}$ NMR, ${ }^{13} \mathrm{C}$ NMR, 2D COSY NMR, 2D COSY NMR, DOSY,}

\section{MALDI-TOF MS and ESI-MS Data}

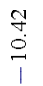

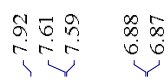
$\underset{\substack{0 \\ i}}{i}$

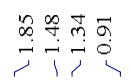
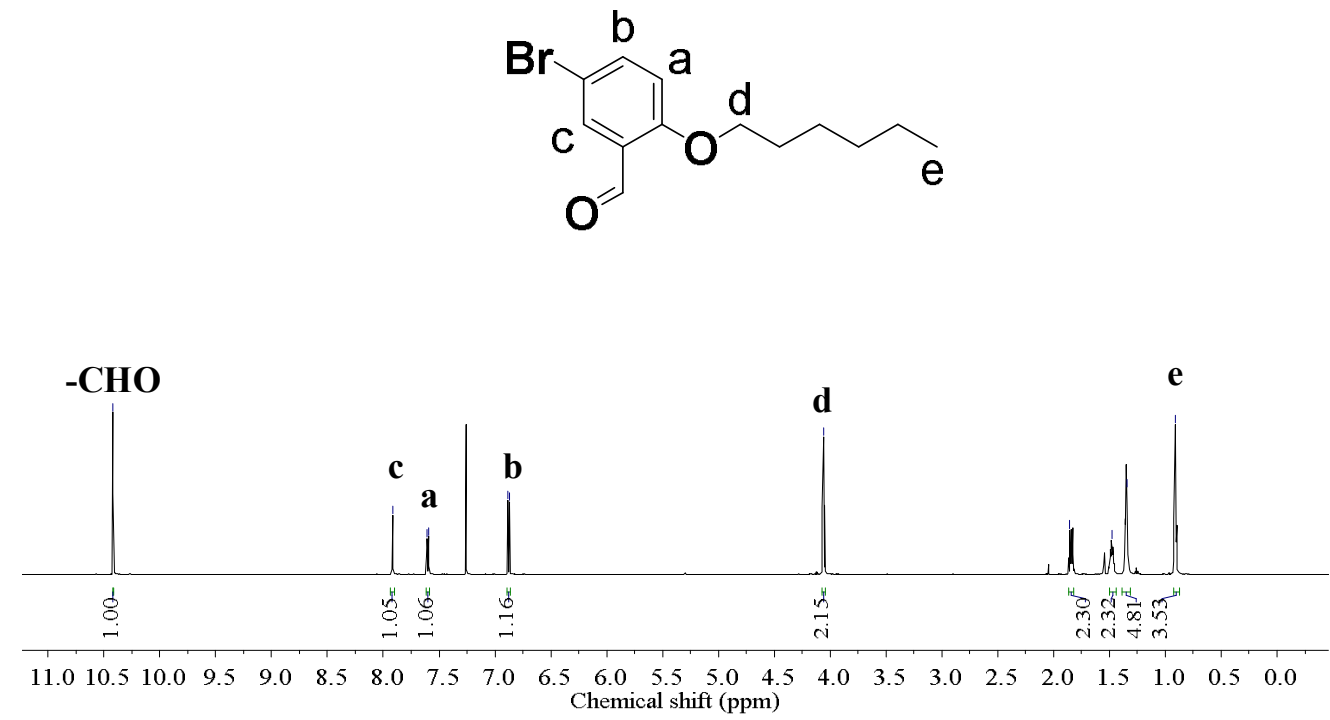

Figure S 1. ${ }^{1} \mathrm{H}$ NMR $\left(600 \mathrm{MHz}, \mathrm{CDCl}_{3}\right)$ spectrum of compound 1.

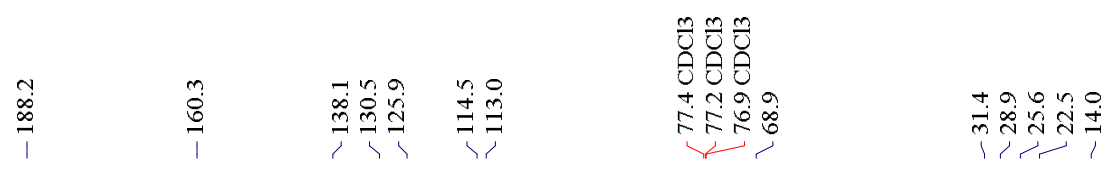

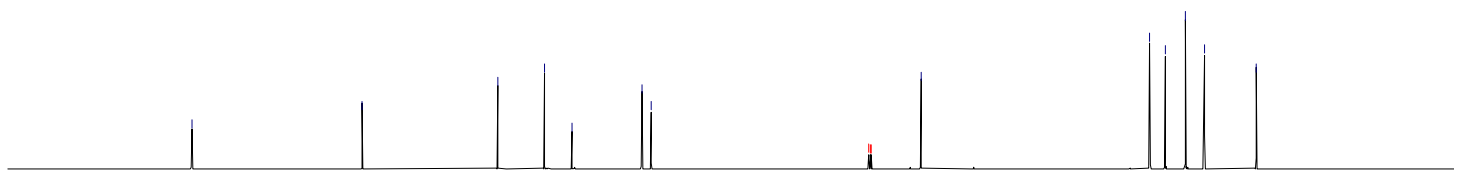

$\begin{array}{llllllllllllllllllllllll}210 & 200 & 190 & 180 & 170 & 160 & 150 & 140 & 130 & 120 & 110 & 100 & 90 & 80 & 70 & 60 & 50 & 40 & 30 & 20 & 10 & 0 & -10\end{array}$ Chemical shift (ppm)

Figure S2. ${ }^{13} \mathrm{C}$ NMR $\left(150 \mathrm{MHz}, \mathrm{CDCl}_{3}\right)$ spectrum of compound $\mathbf{1}$. 


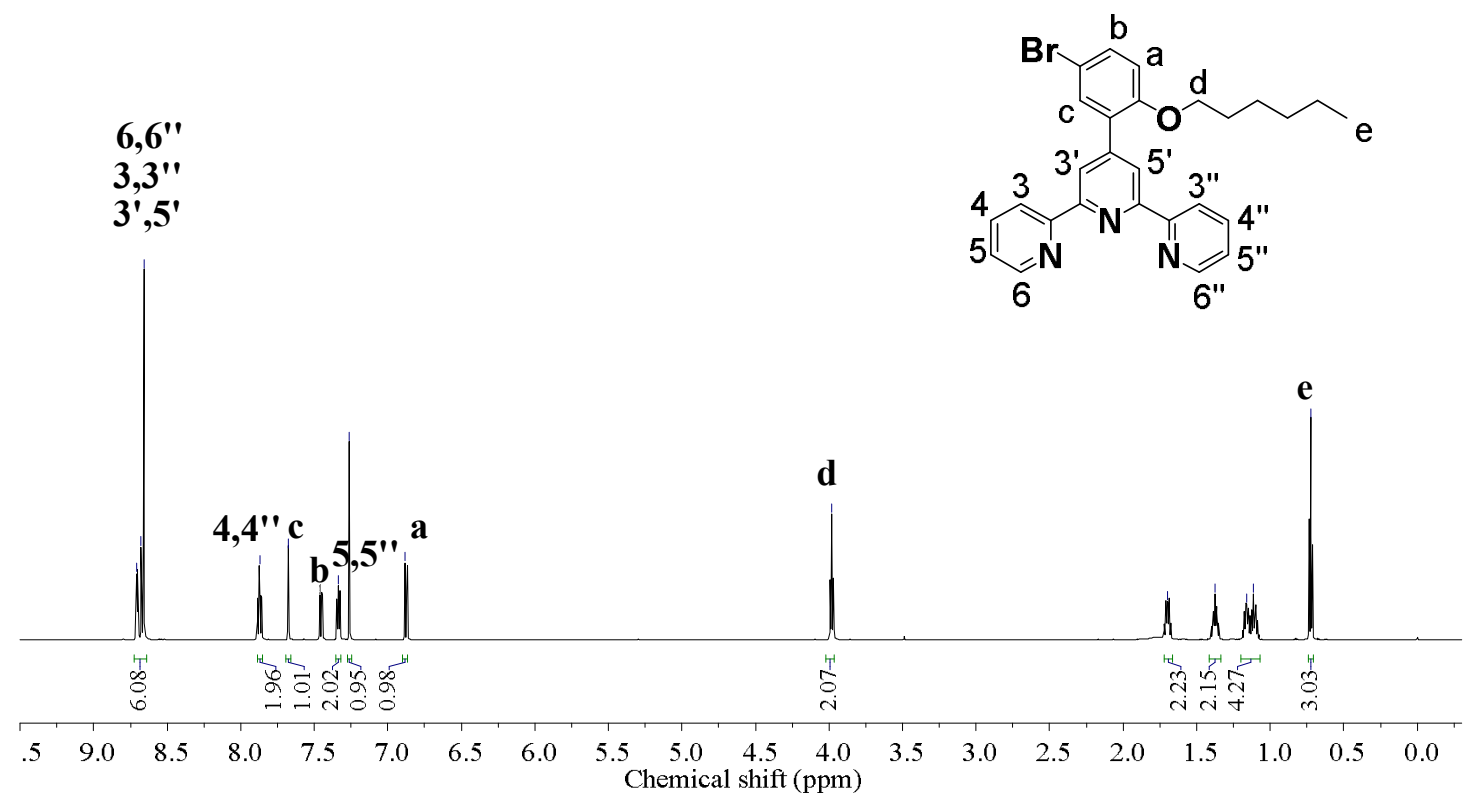

Figure S3. ${ }^{1} \mathrm{H}$ NMR $\left(600 \mathrm{MHz}, \mathrm{CDCl}_{3}\right)$ spectrum of compound 2.

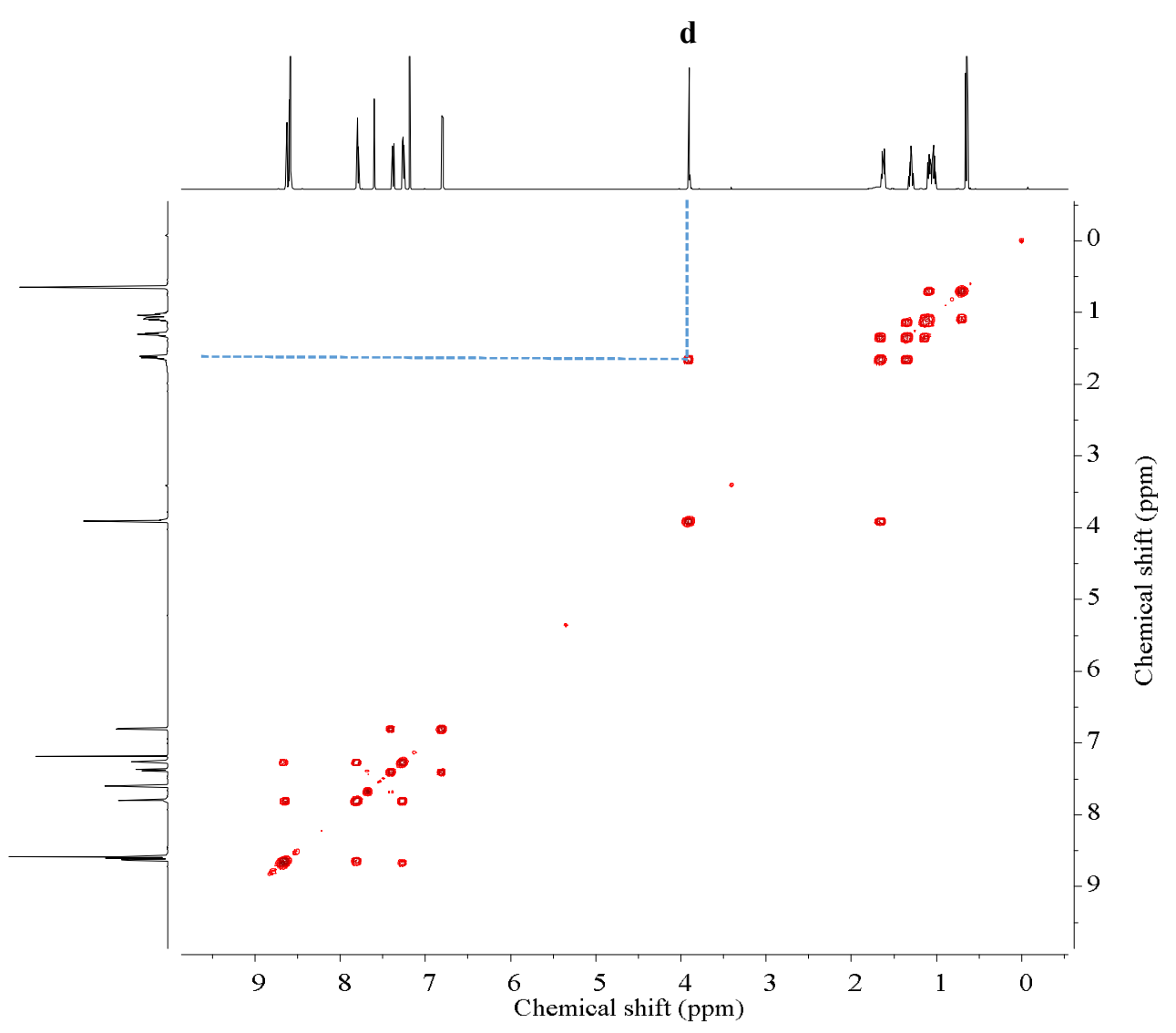

Figure S4. 2D COSY NMR (600 MHz, $\left.\mathrm{CDCl}_{3}\right)$ spectrum of compound 2. 


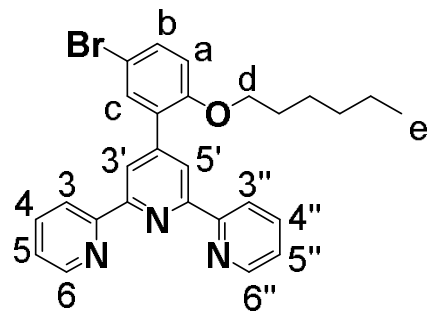

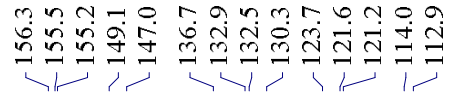

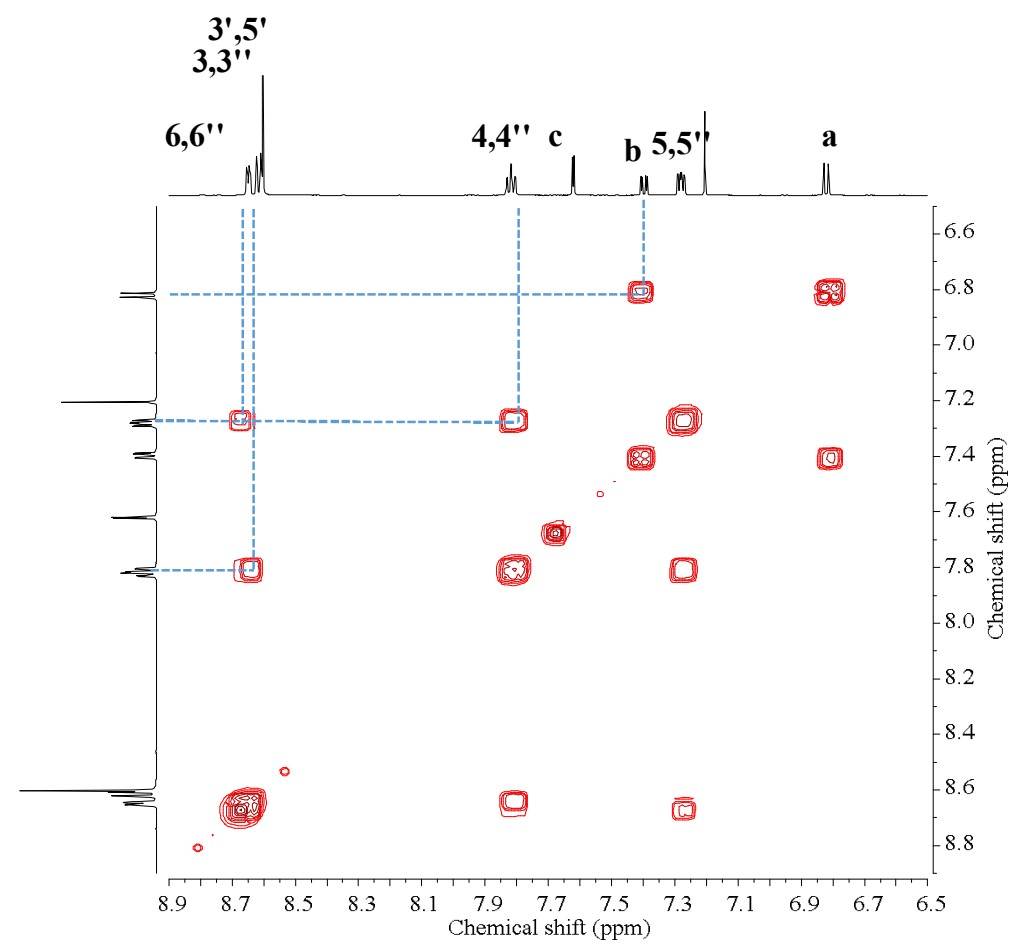

Figure S5. 2D COSY NMR (600 MHz, $\left.\mathrm{CDCl}_{3}\right)$ spectrum of compound 2 (aromatic region).
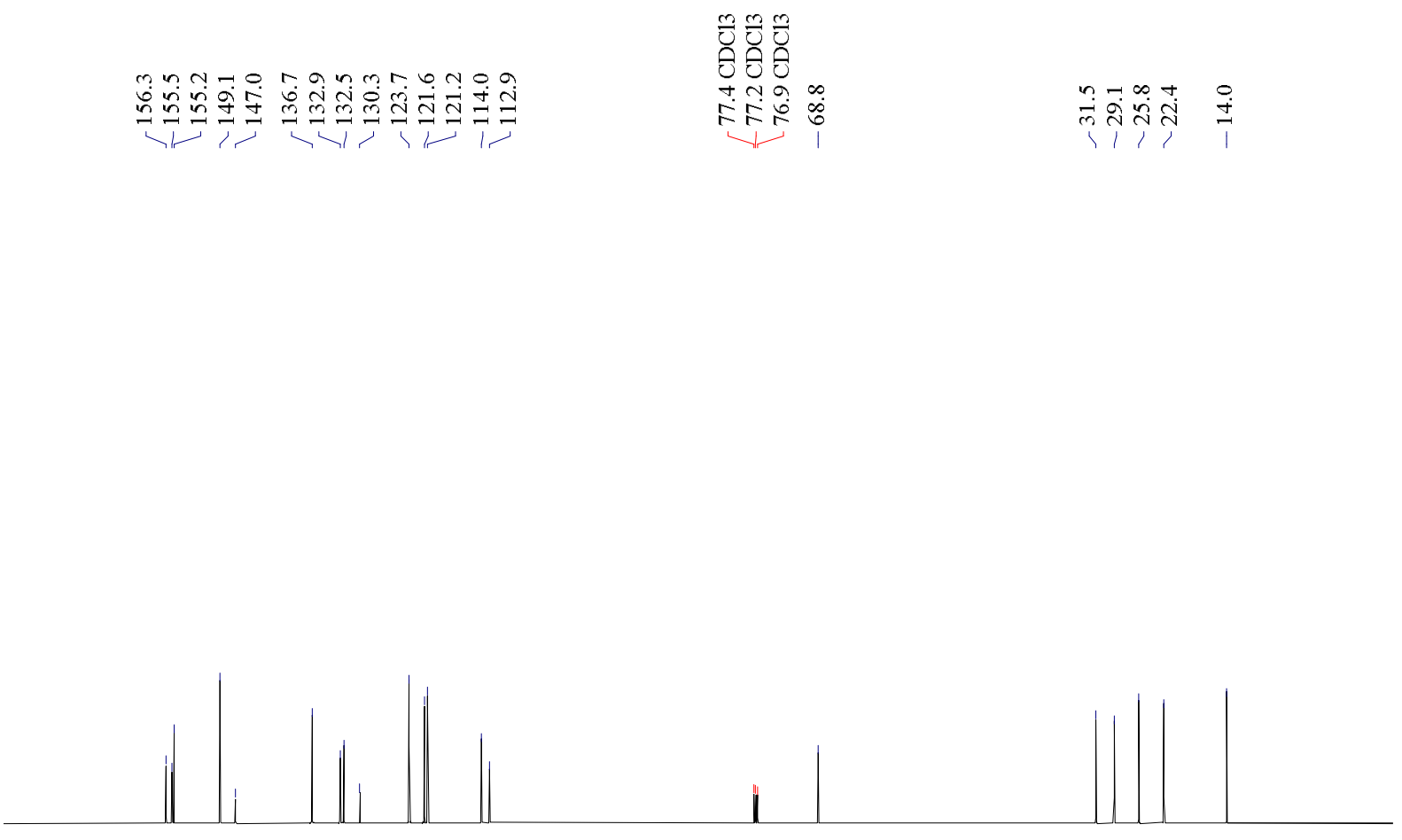

$\begin{array}{llllllllllllllllll}170 & 160 & 150 & 140 & 130 & 120 & 110 & 100 & 90 & 80 & 70 & 60 & 50 & 40 & 30 & 20 & 10 & 0 \\ \text { Chemical shift (ppm) }\end{array}$

Figure S6. ${ }^{13} \mathrm{C}$ NMR $\left(150 \mathrm{MHz}, \mathrm{CDCl}_{3}\right)$ spectrum of compound 2. 

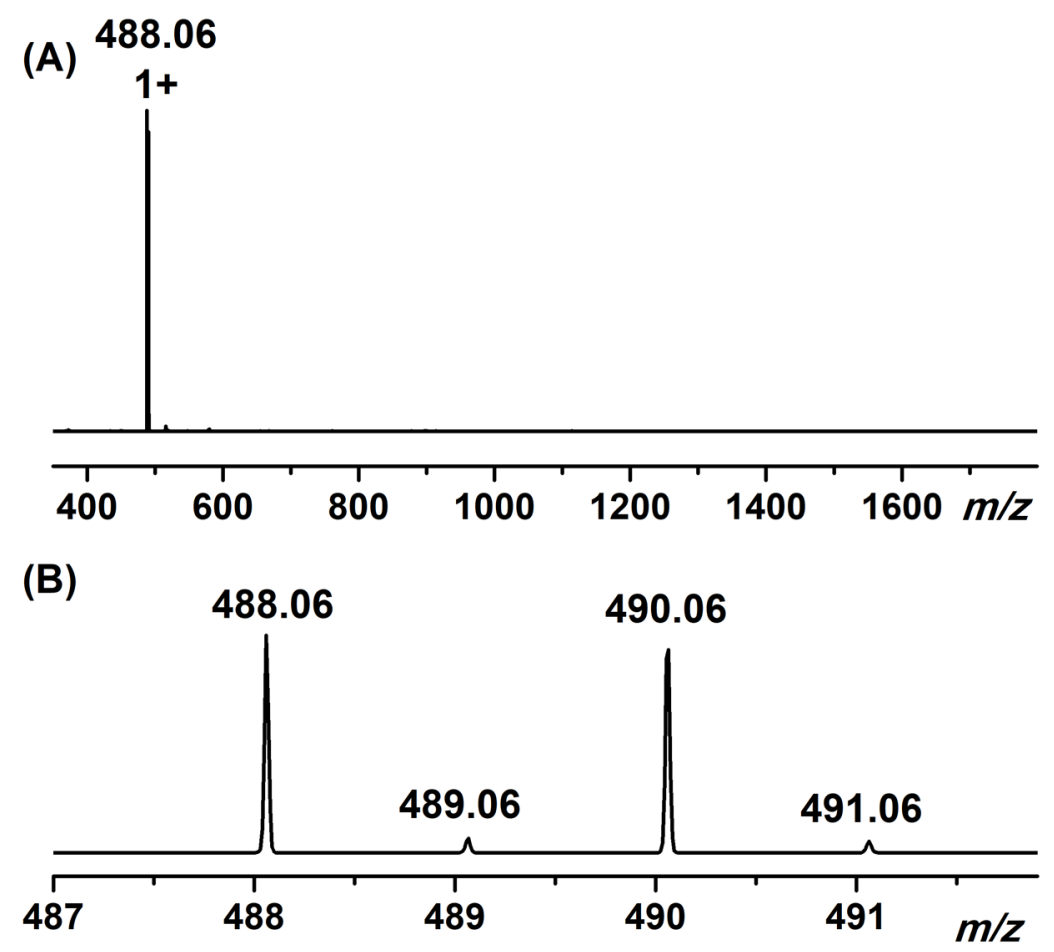

Figure S7. (A) ESI-MS spectrum of compound 2 in $\mathrm{CHCl}_{3} / \mathrm{CH}_{3} \mathrm{OH}(1 / 3)$ and (B) isotope pattern.

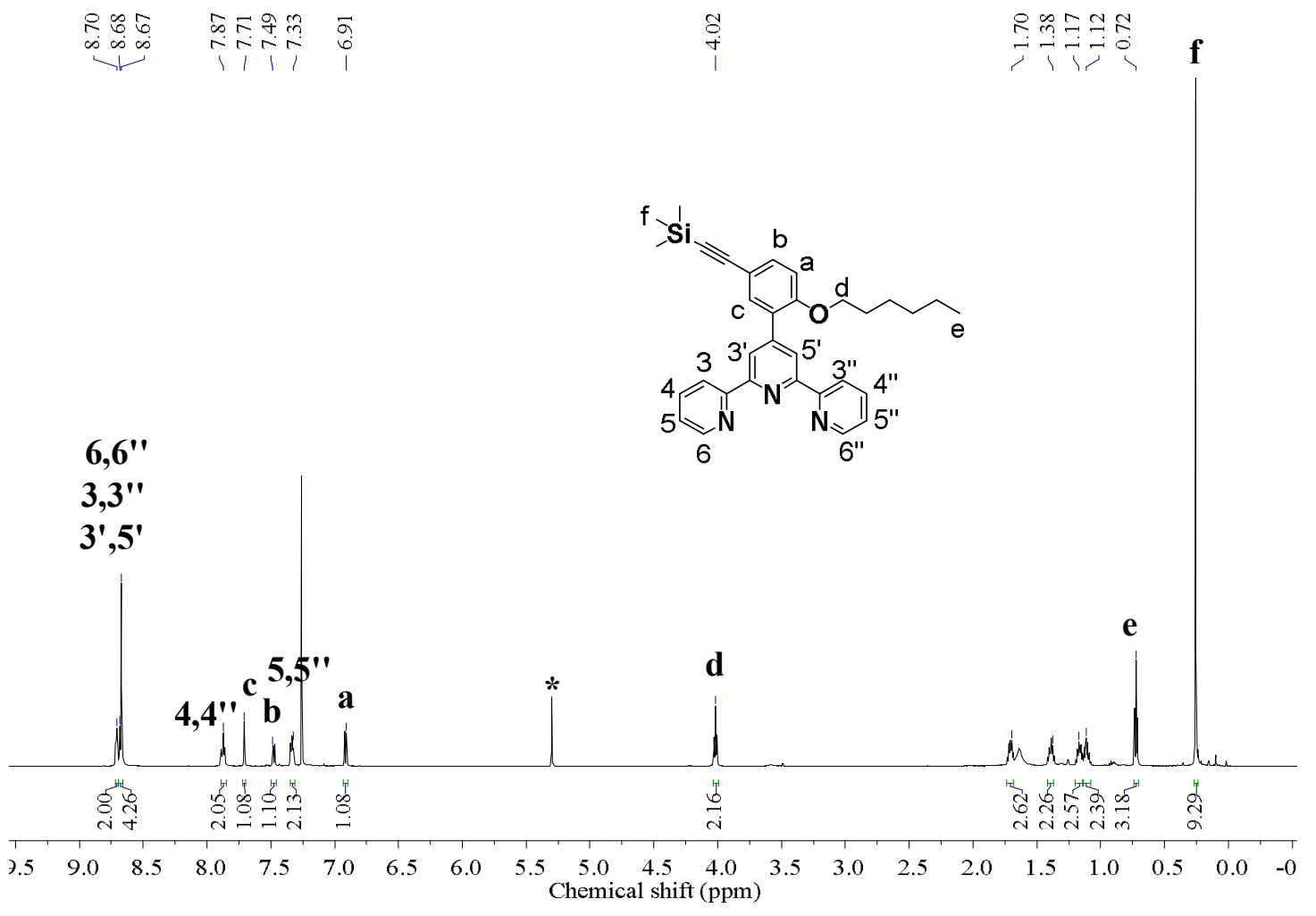

Figure S8. ${ }^{1} \mathrm{H}$ NMR $\left(600 \mathrm{MHz}, \mathrm{CDCl}_{3}\right)$ spectrum of compound $3\left({ }^{*}-\mathrm{CH}_{2} \mathrm{Cl}_{2}\right)$. 


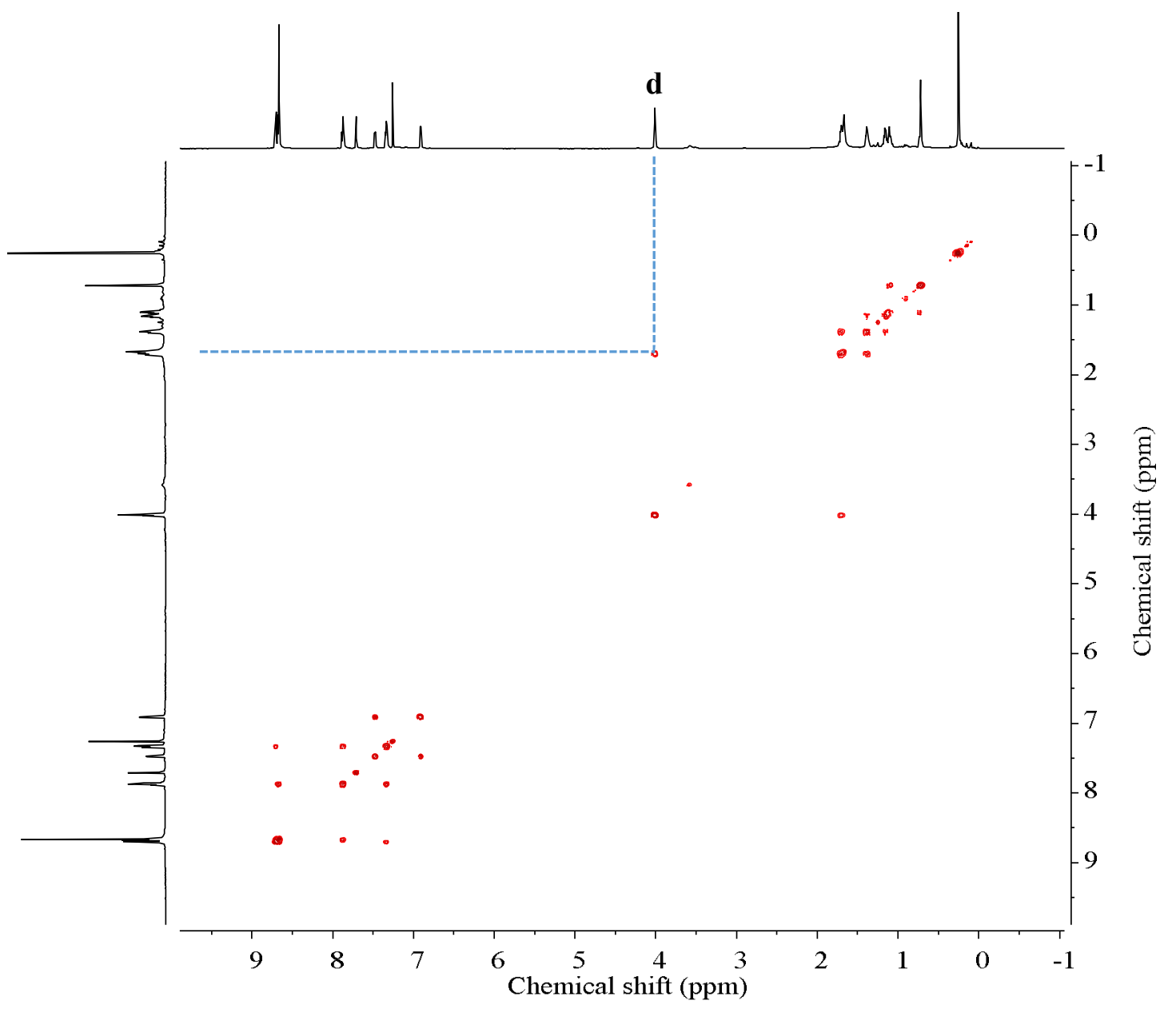

Figure S9. 2D COSY NMR (600 MHz, $\left.\mathrm{CDCl}_{3}\right)$ spectrum of compound 3.

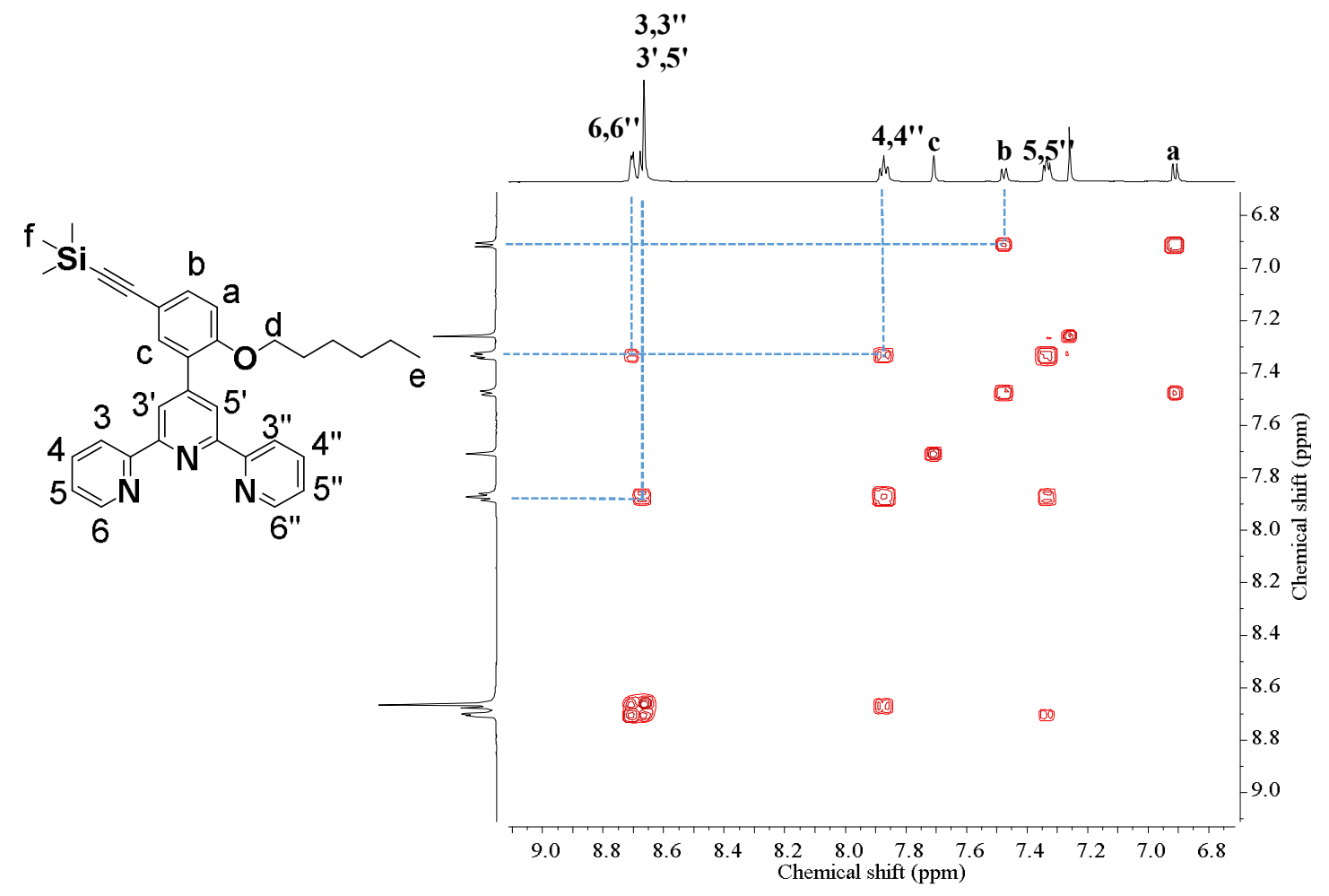

Figure S10. 2D COSY NMR (600 MHz, $\left.\mathrm{CDCl}_{3}\right)$ spectrum of compound 3 (aromatic region). 


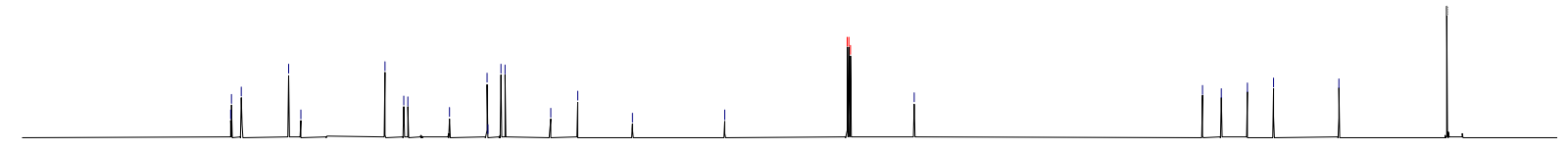

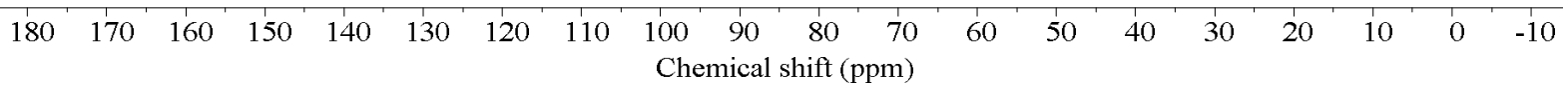

Figure S11. ${ }^{13} \mathrm{C}$ NMR $\left(150 \mathrm{MHz}, \mathrm{CDCl}_{3}\right)$ spectrum of compound 3.

(A)

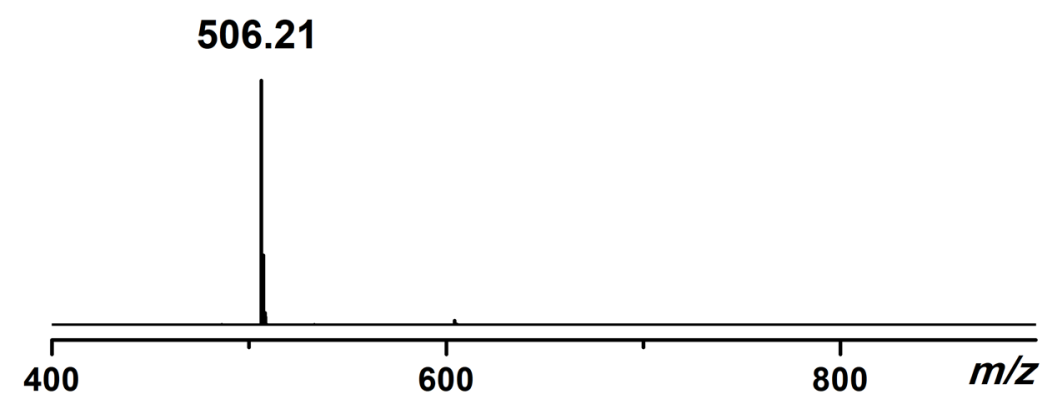

(B)

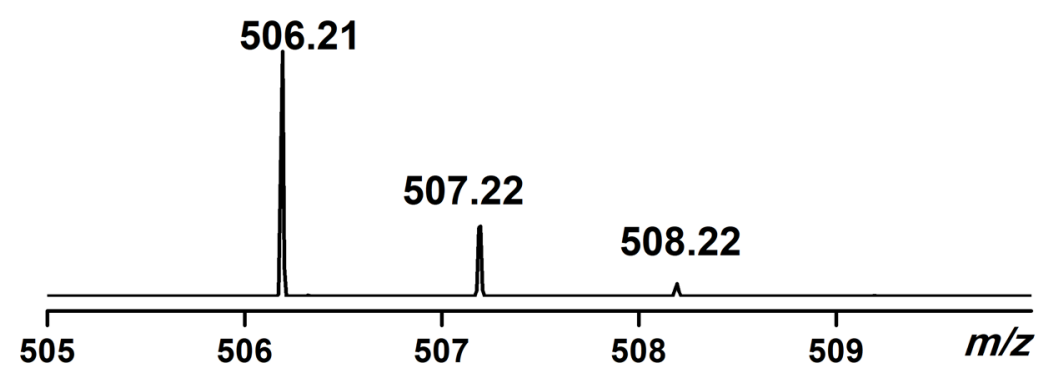

Figure S12. (A) ESI-MS spectrum of compound 3 in $\mathrm{CHCl}_{3} / \mathrm{CH}_{3} \mathrm{OH}(1 / 3)$ and (B) isotope pattern. 


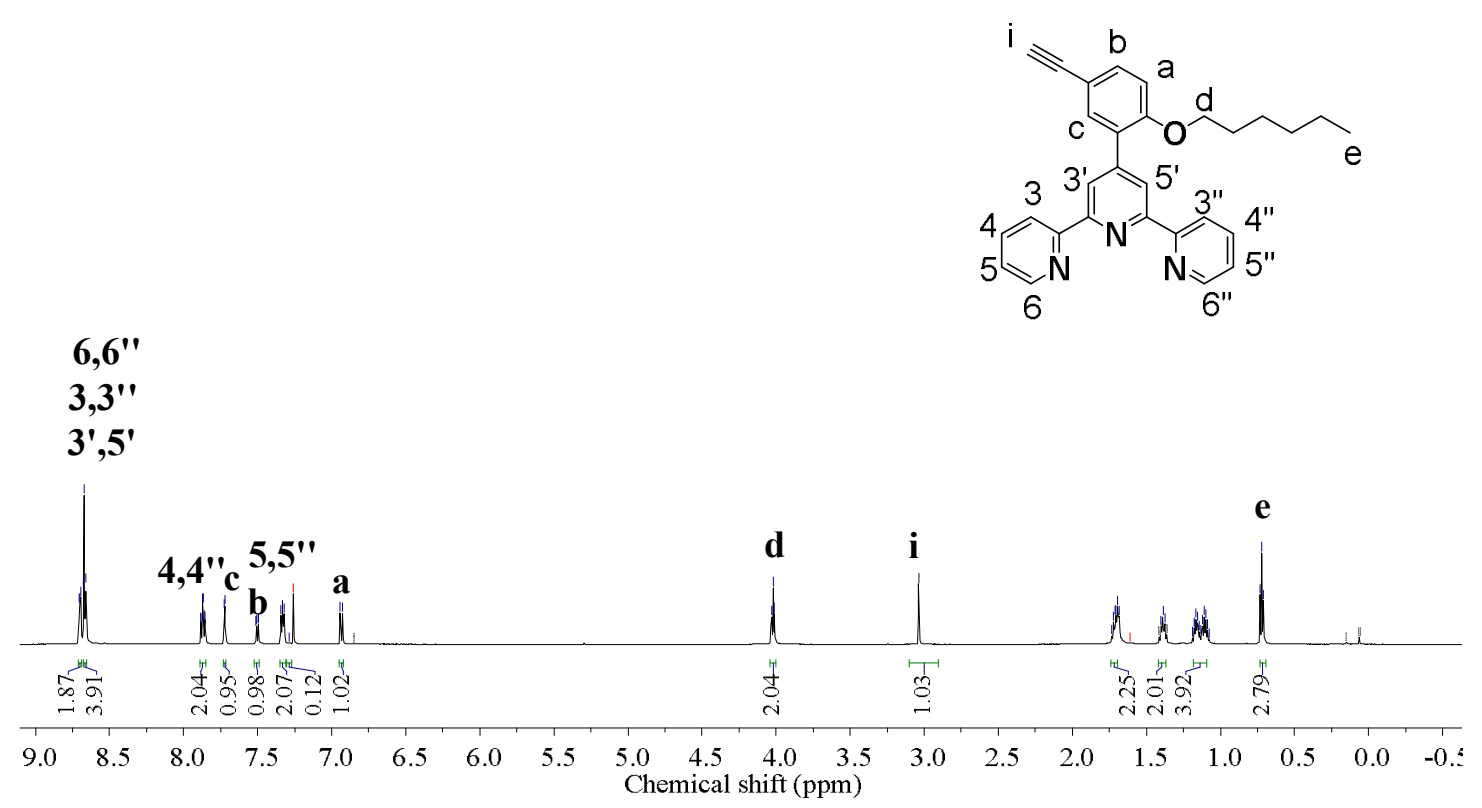

Figure S13. ${ }^{1} \mathrm{H}$ NMR $\left(600 \mathrm{MHz}, \mathrm{CDCl}_{3}\right)$ spectrum of compound 4.

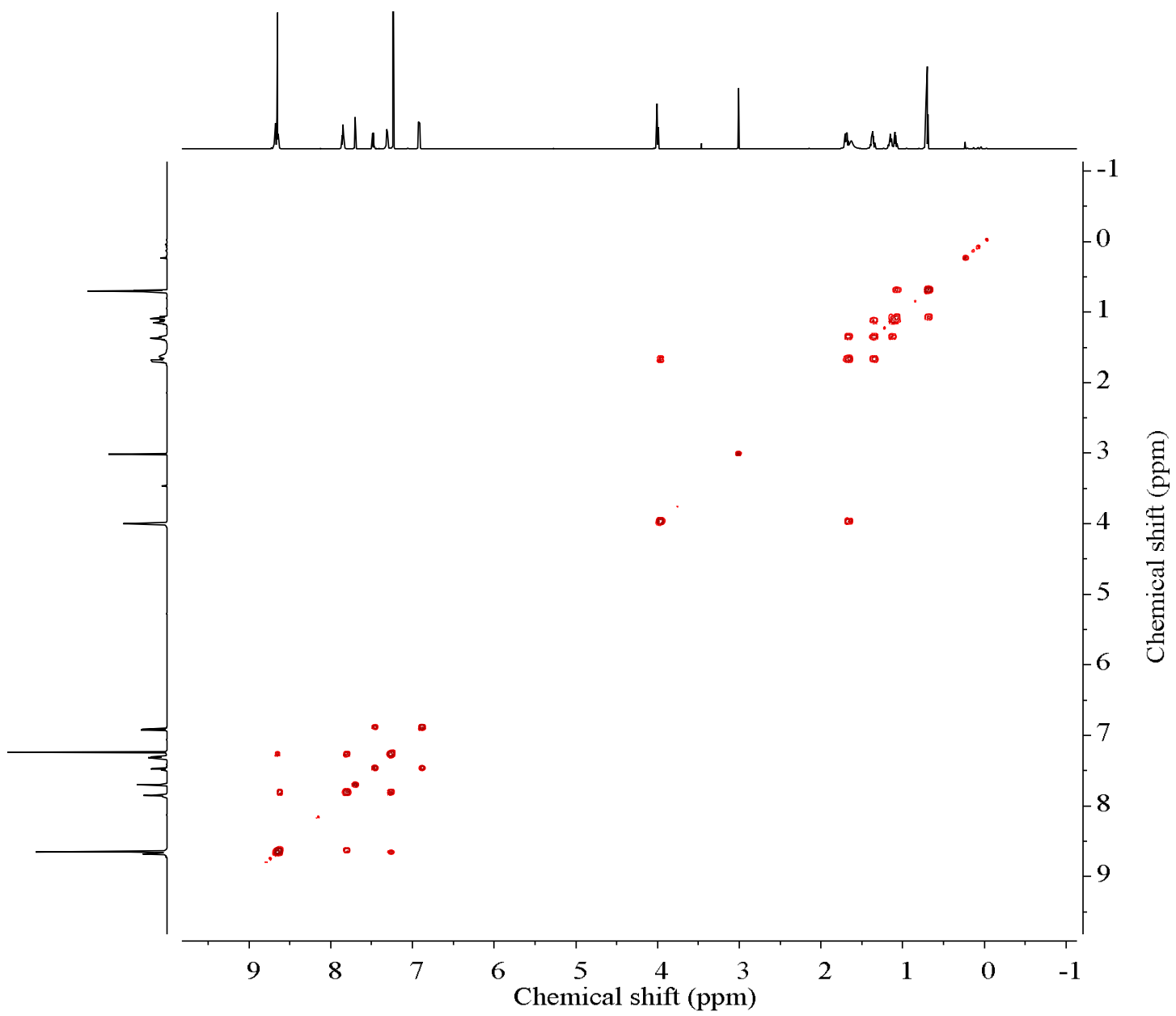

Figure S14. 2D COSY NMR (600 MHz, $\left.\mathrm{CDCl}_{3}\right)$ spectrum of compound 4. 


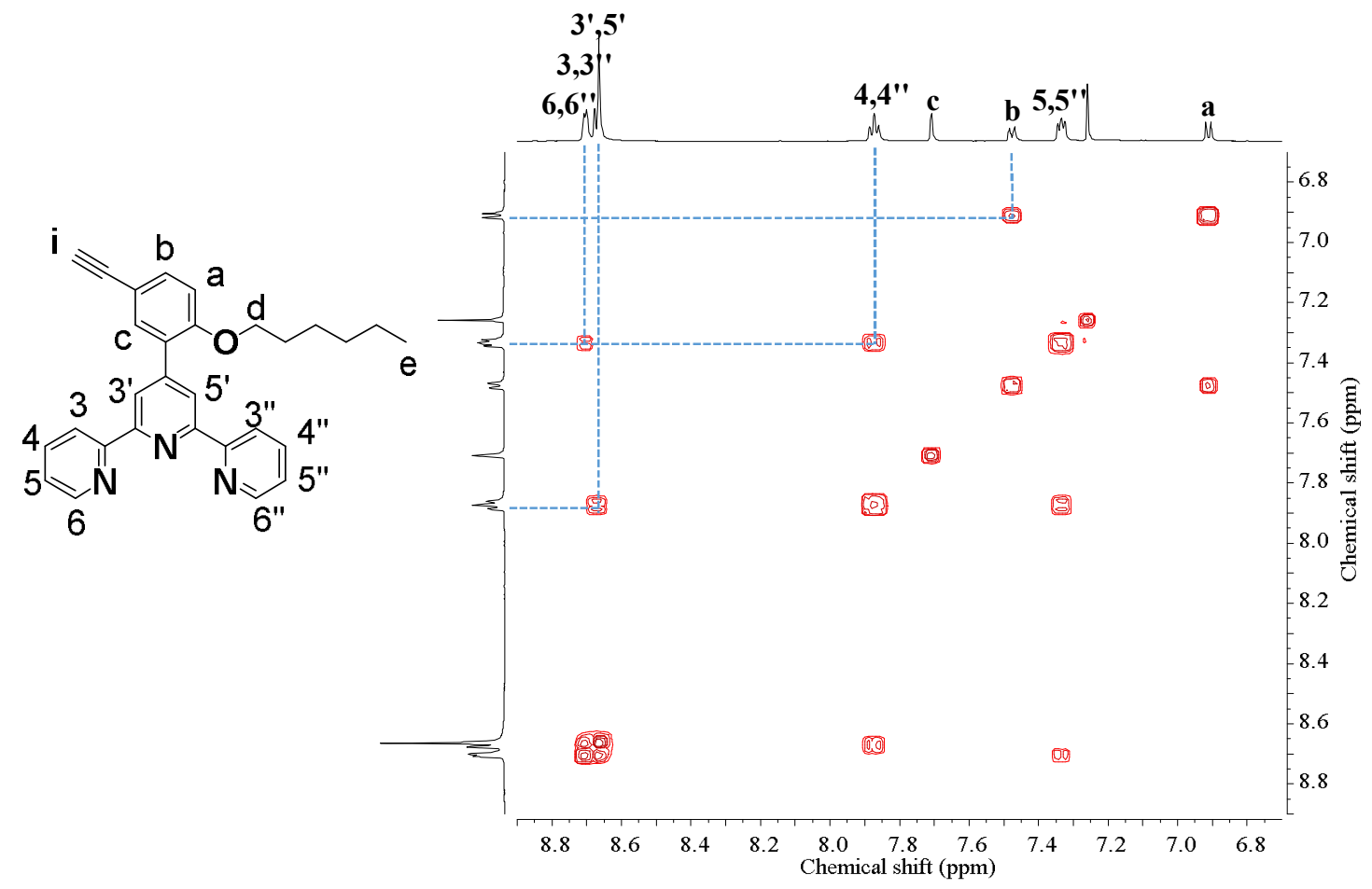

Figure S15. 2D COSY NMR (600 MHz, $\mathrm{CDCl}_{3}$ ) spectrum of compound 4 (aromatic region).

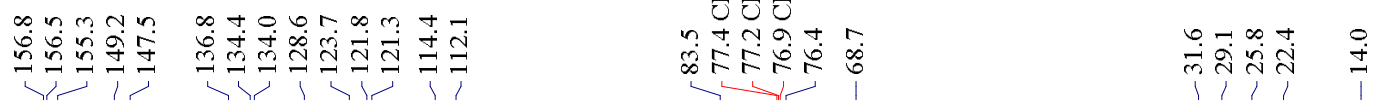

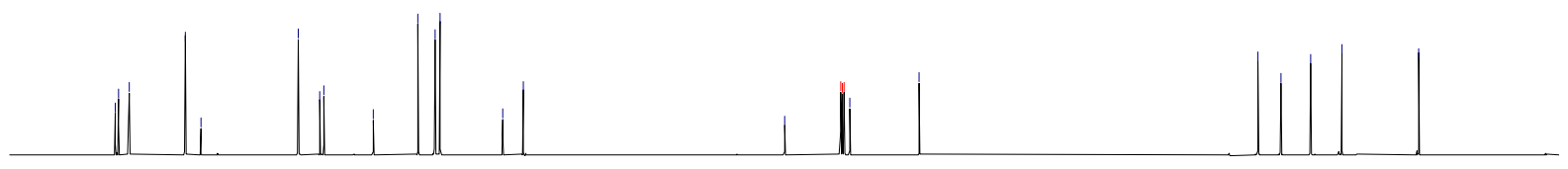

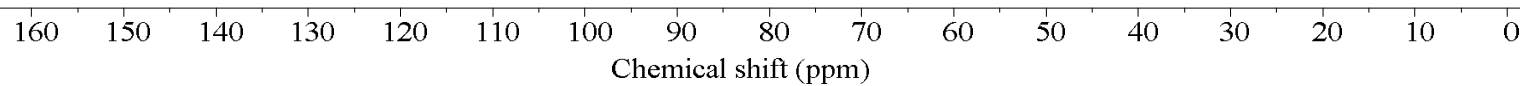

Figure S16. ${ }^{13} \mathrm{C}$ NMR $\left(150 \mathrm{MHz}, \mathrm{CDCl}_{3}\right)$ spectrum of compound 4. 


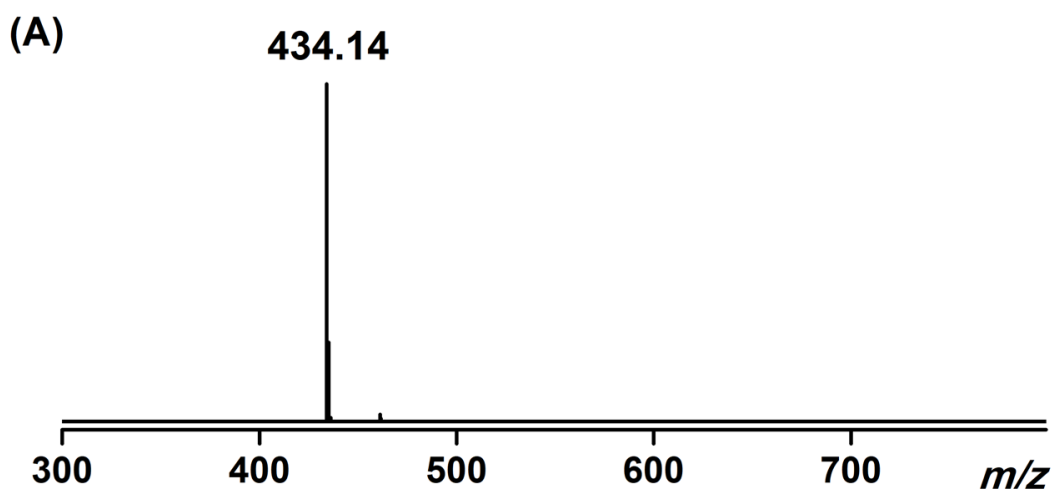

(B)

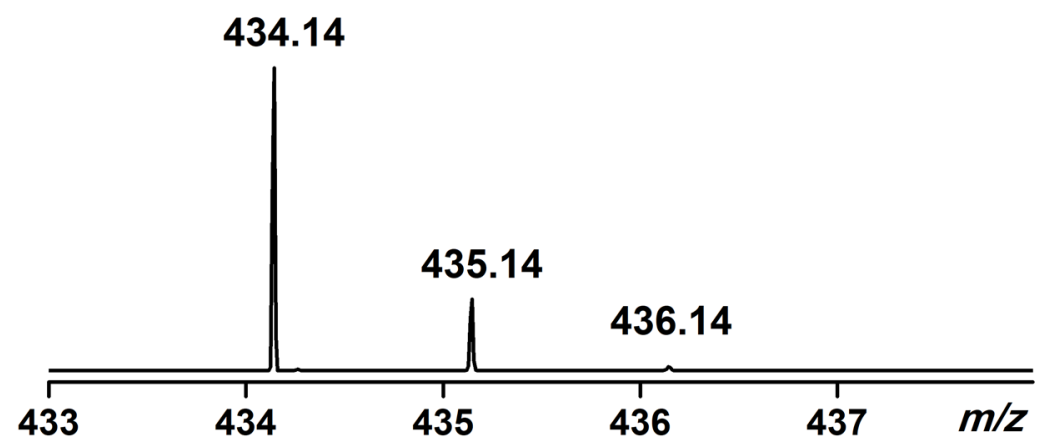

Figure S17. (A) ESI-MS spectrum of compound 4 in $\mathrm{CHCl}_{3} / \mathrm{CH}_{3} \mathrm{OH}(1 / 3)$ and (B) isotope pattern.

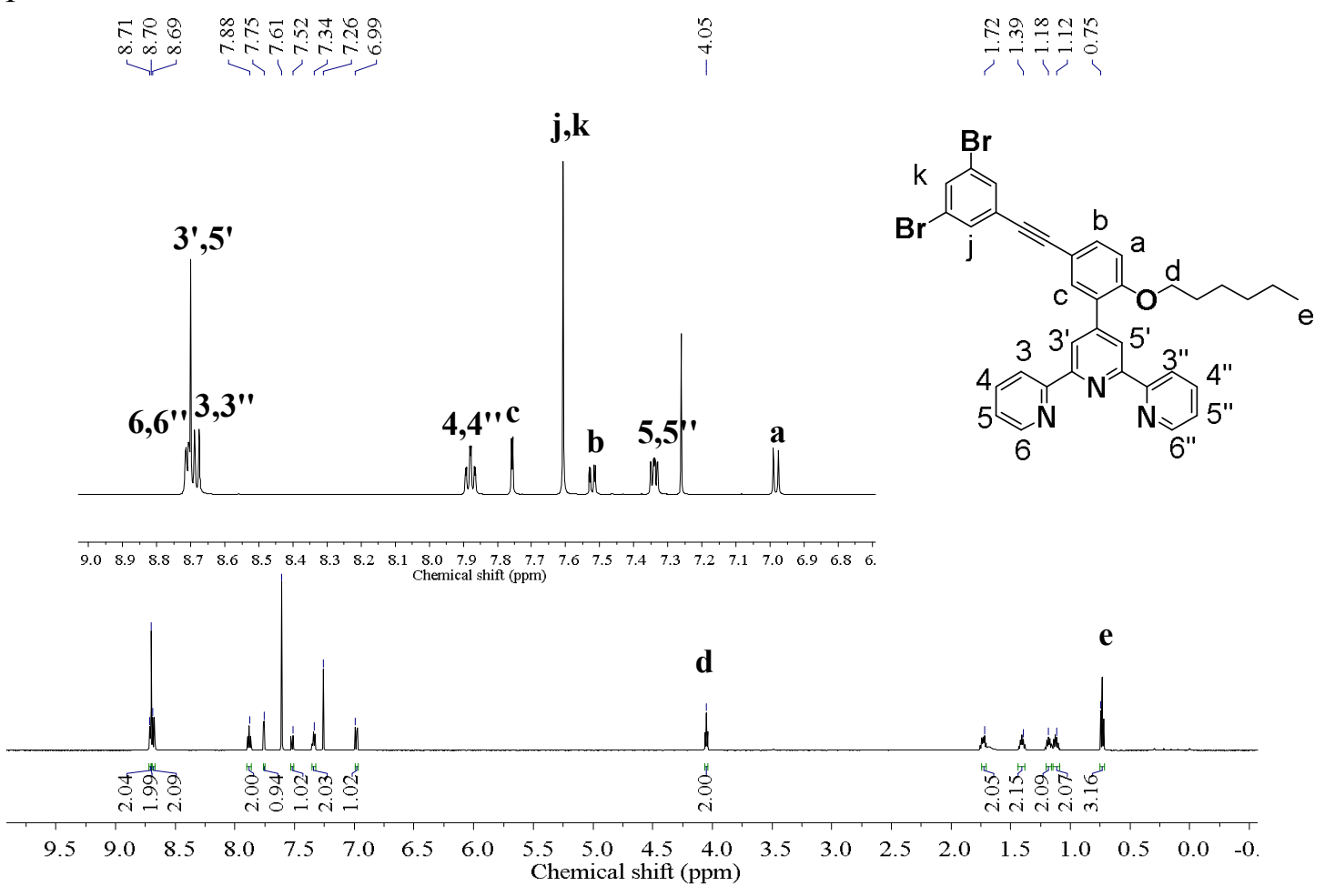

Figure S18. ${ }^{1} \mathrm{H} \mathrm{NMR}\left(600 \mathrm{MHz}, \mathrm{CDCl}_{3}\right)$ spectrum of compound 5. 


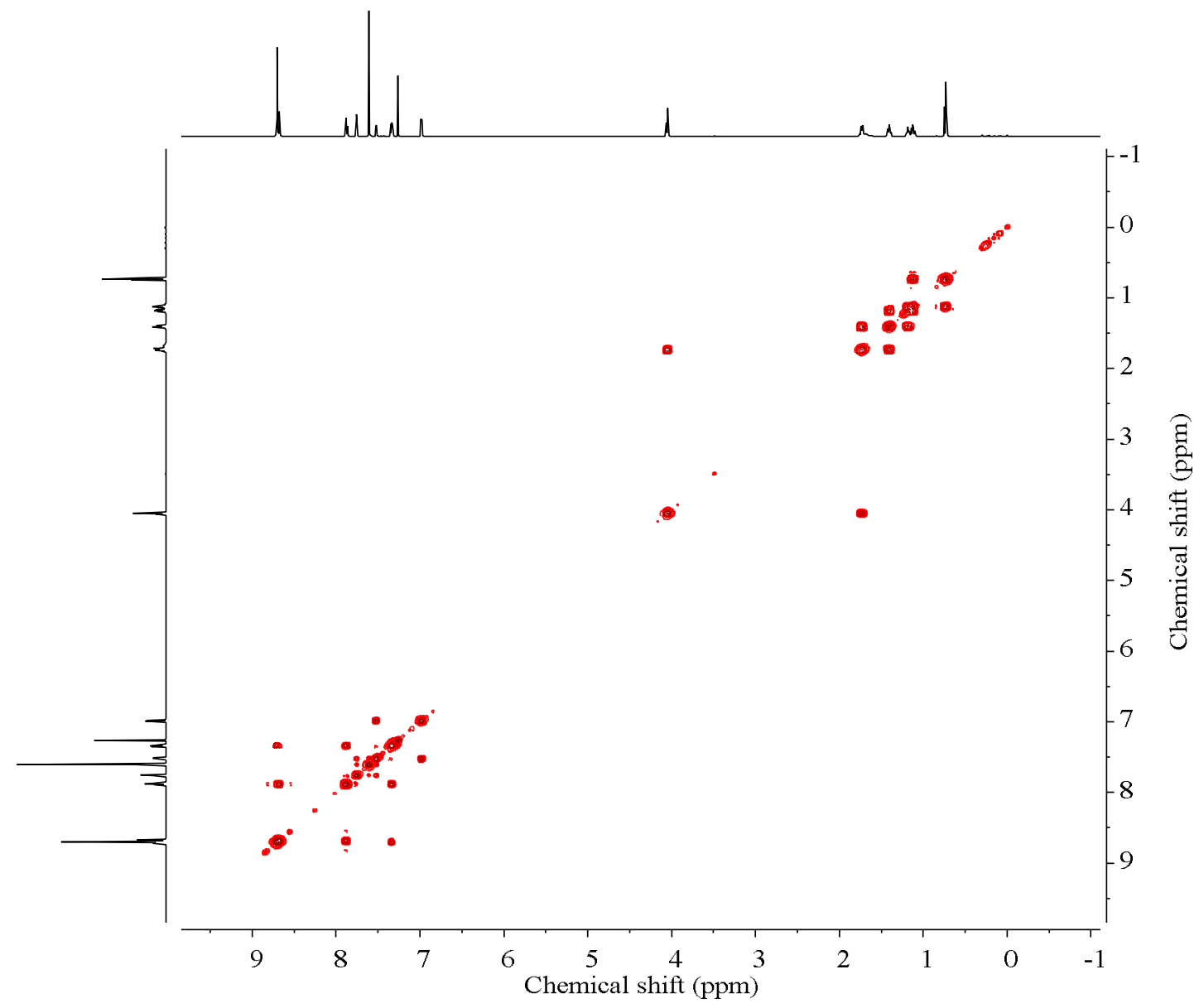

Figure S19. 2D COSY NMR (600 MHz, $\left.\mathrm{CDCl}_{3}\right)$ spectrum of compound 5.

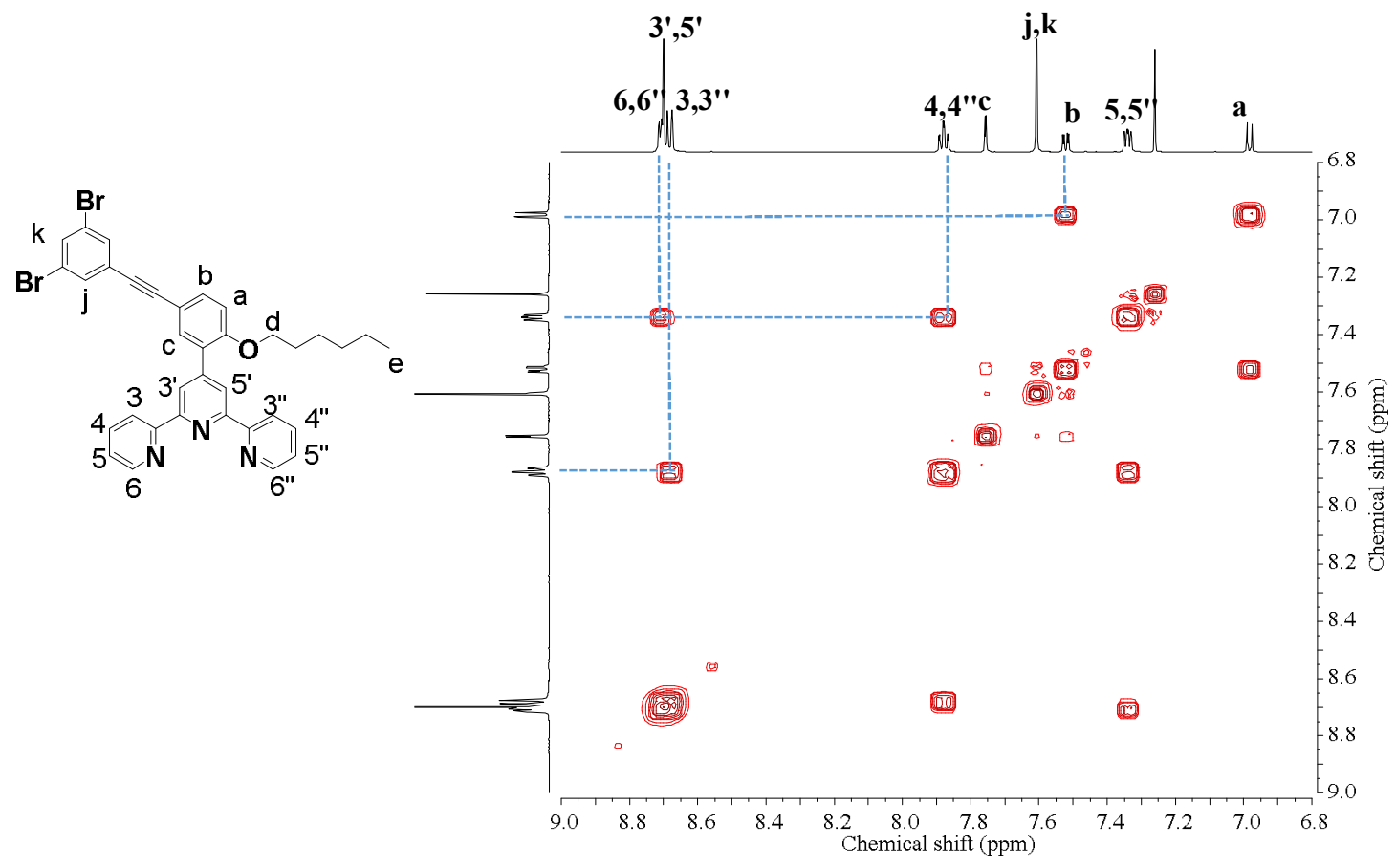

Figure S20. 2D COSY NMR (600 MHz, $\mathrm{CDCl}_{3}$ ) spectrum of compound 5 (aromatic region). 


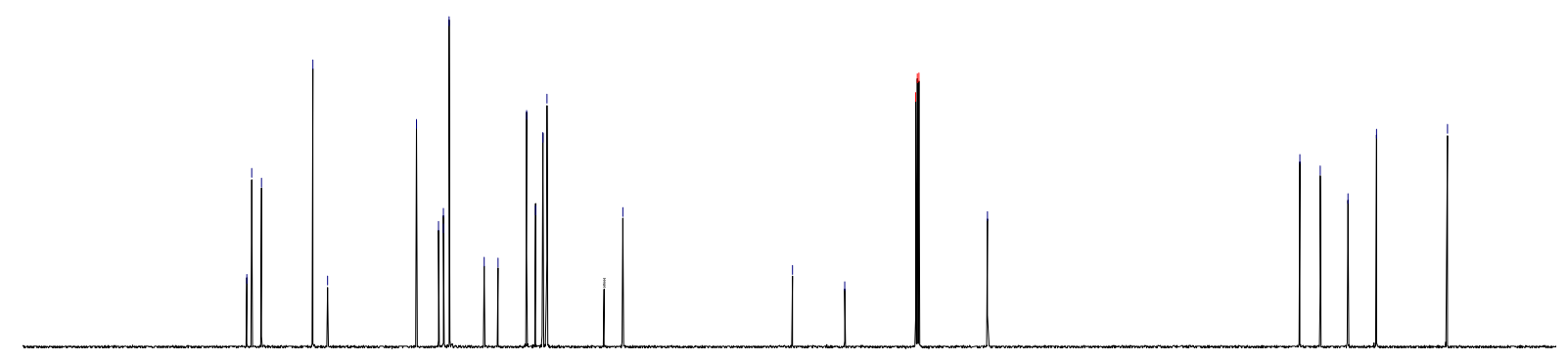

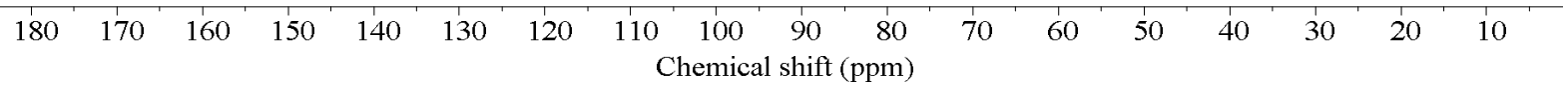

Figure S21. ${ }^{13} \mathrm{C}$ NMR $\left(150 \mathrm{MHz}, \mathrm{CDCl}_{3}\right)$ spectrum of compound 5.

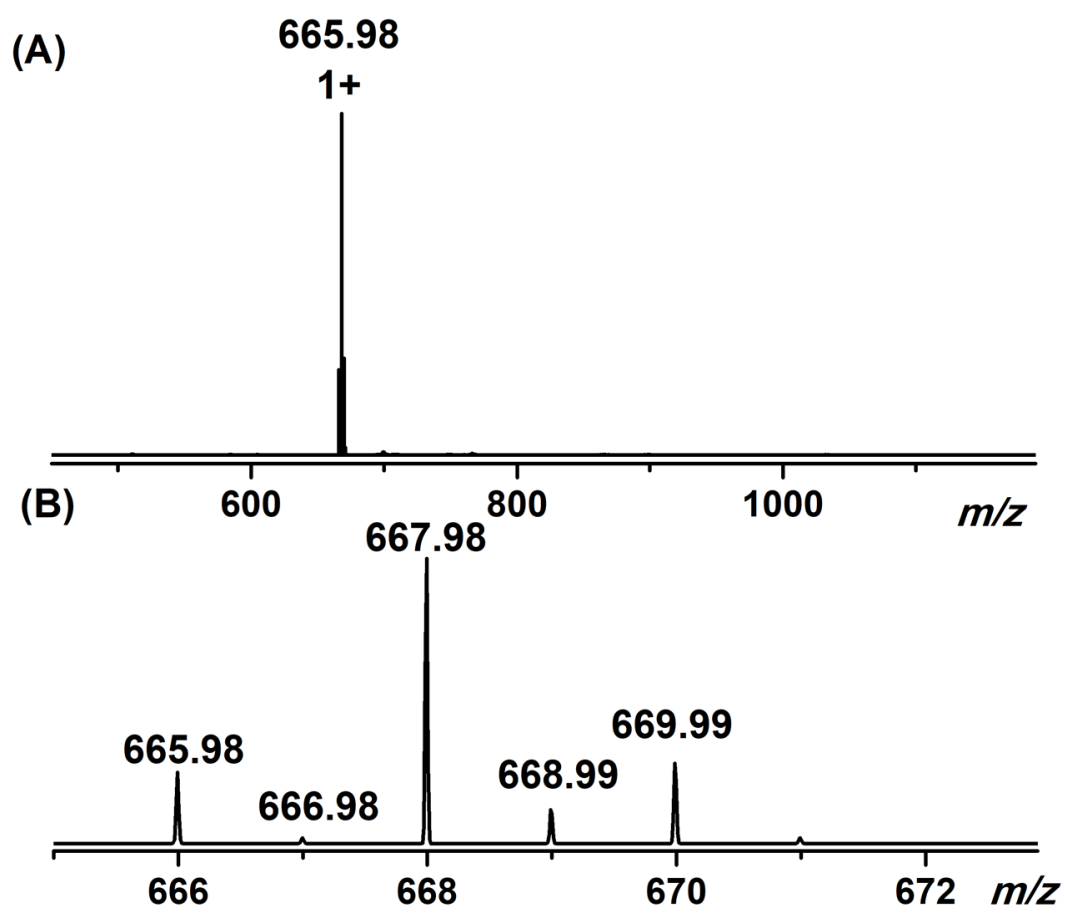

Figure S22. (A) ESI-MS spectrum of compound 5 in $\mathrm{CHCl}_{3} / \mathrm{CH}_{3} \mathrm{OH}(1 / 3)$ and (B) isotope pattern. 


$$
3^{\prime}, 5^{\prime}
$$
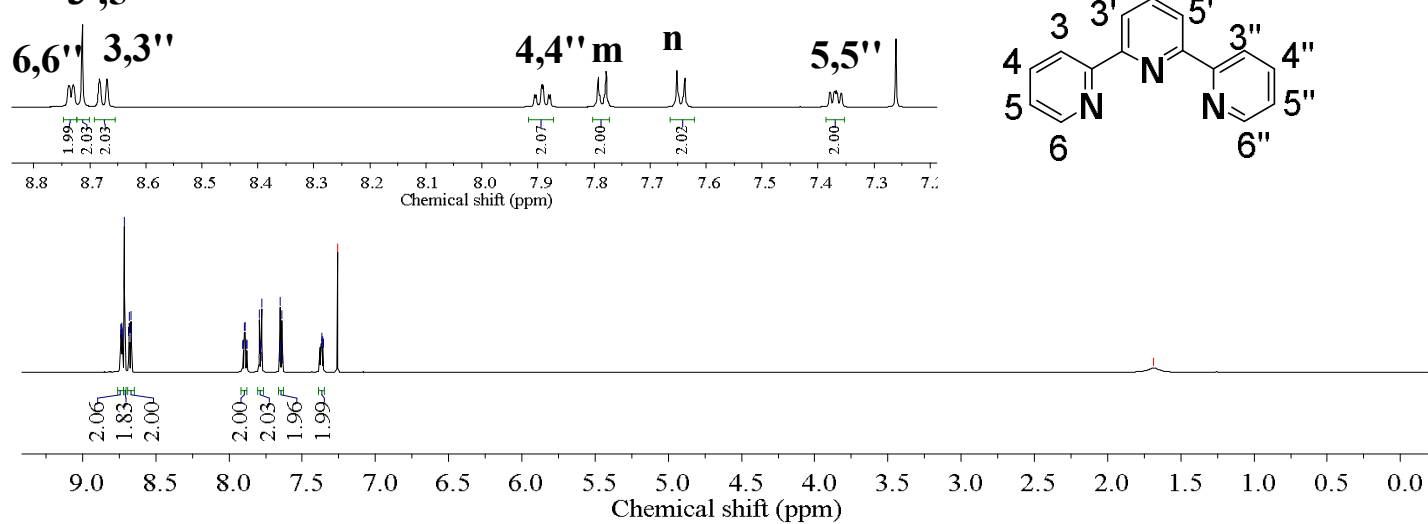

Figure S23. ${ }^{1} \mathrm{H} \mathrm{NMR}\left(600 \mathrm{MHz}, \mathrm{CDCl}_{3}\right)$ spectrum of compound 6.

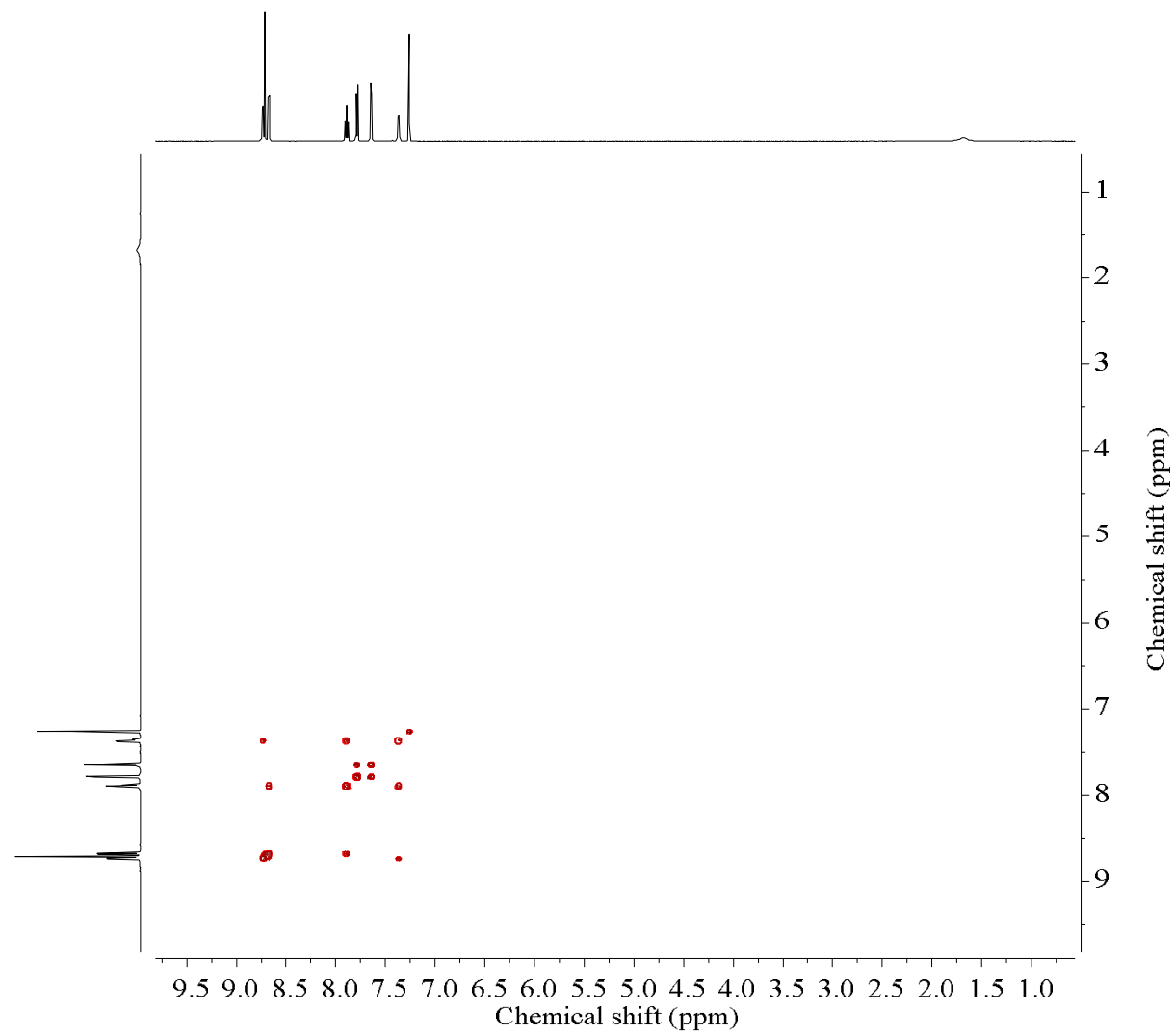

Figure S24. 2D COSY NMR (600 MHz, $\left.\mathrm{CDCl}_{3}\right)$ spectrum of compound 6. 


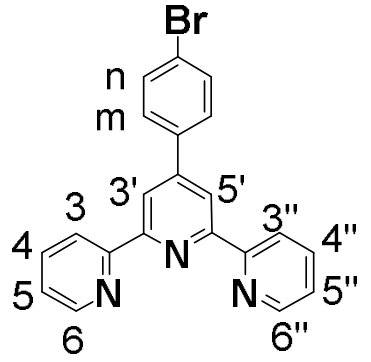

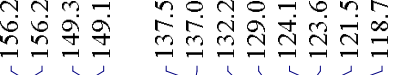

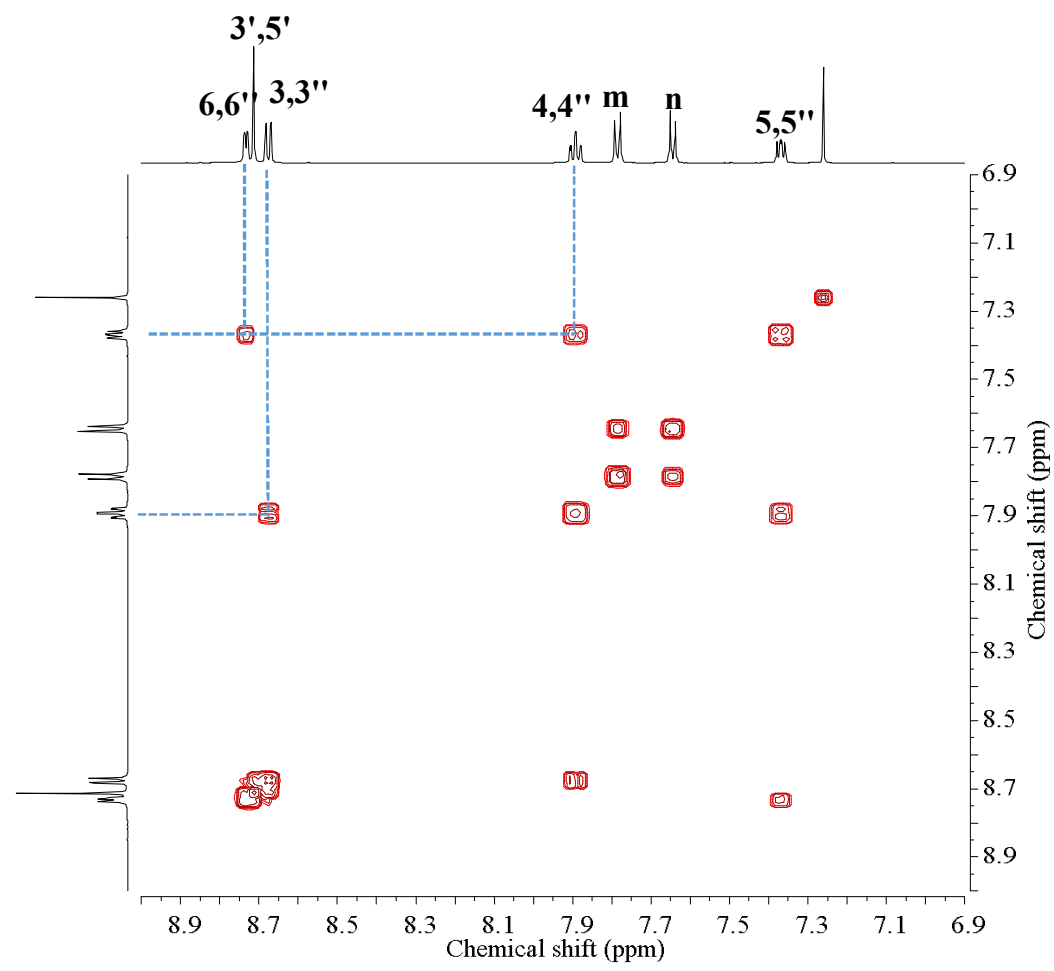

Figure S25. 2D COSY NMR (600 MHz, $\left.\mathrm{CDCl}_{3}\right)$ spectrum of compound 6 (aromatic region).

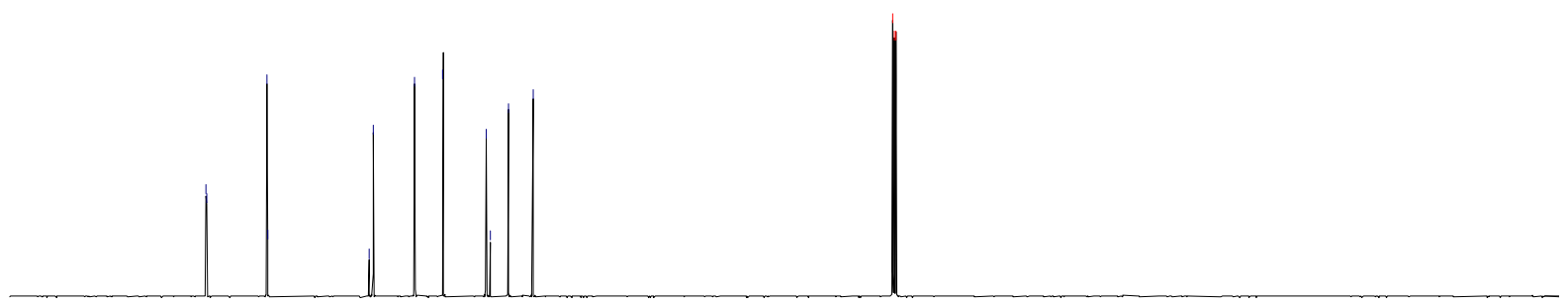

$\begin{array}{lllllllllllllllll}170 & 160 & 150 & 140 & 130 & 120 & 110 & 100 & 90 & 80 & 70 & 60 & 50 & 40 & 30 & 20 & 10\end{array}$

Figure S26. ${ }^{13} \mathrm{C}$ NMR $\left(150 \mathrm{MHz} \mathrm{CDCl}_{3}\right)$ spectrum of compound 6. 


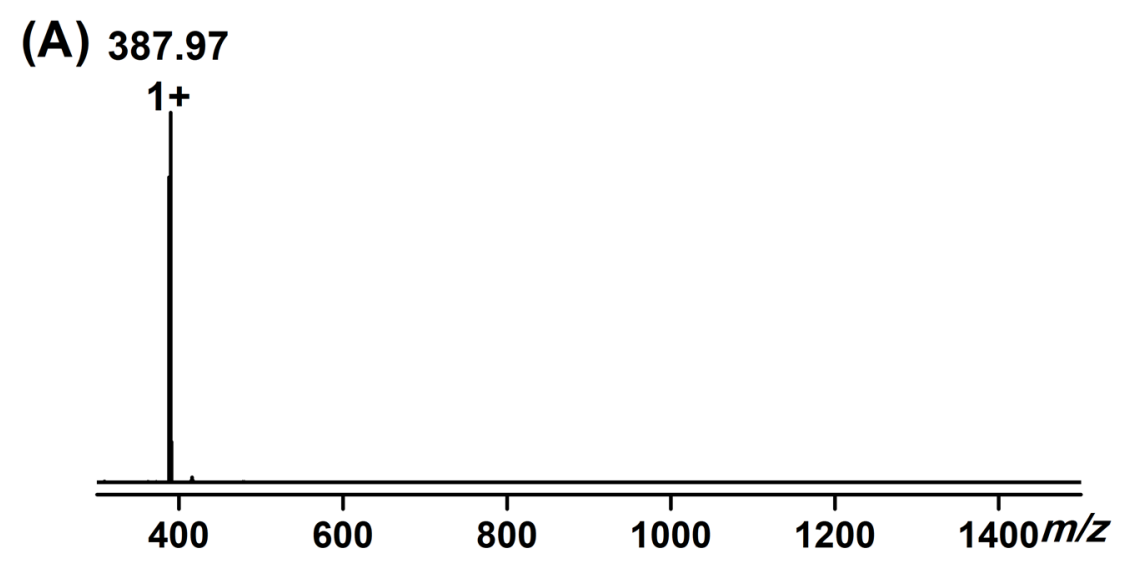

(B)

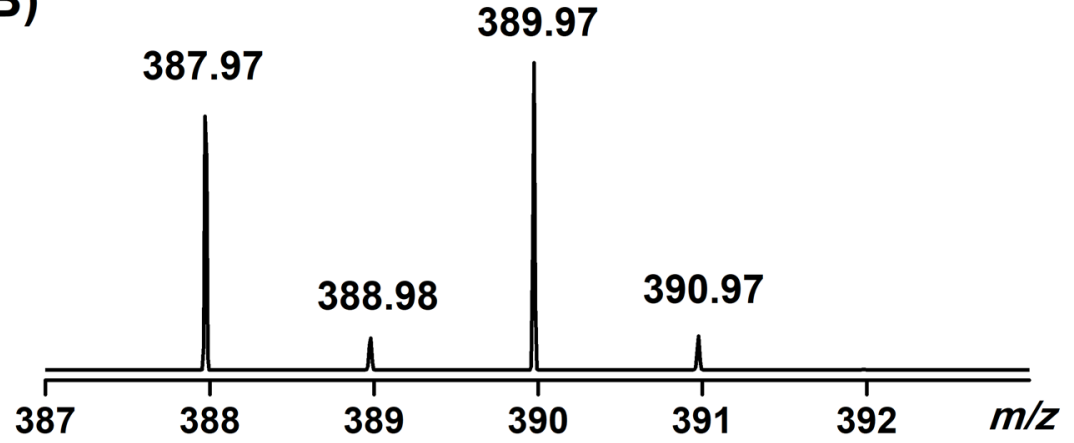

Figure S27. (A) ESI-MS spectrum of compound 6 in $\mathrm{CHCl}_{3} / \mathrm{CH}_{3} \mathrm{OH}(1 / 3)$ and (B) isotope pattern.

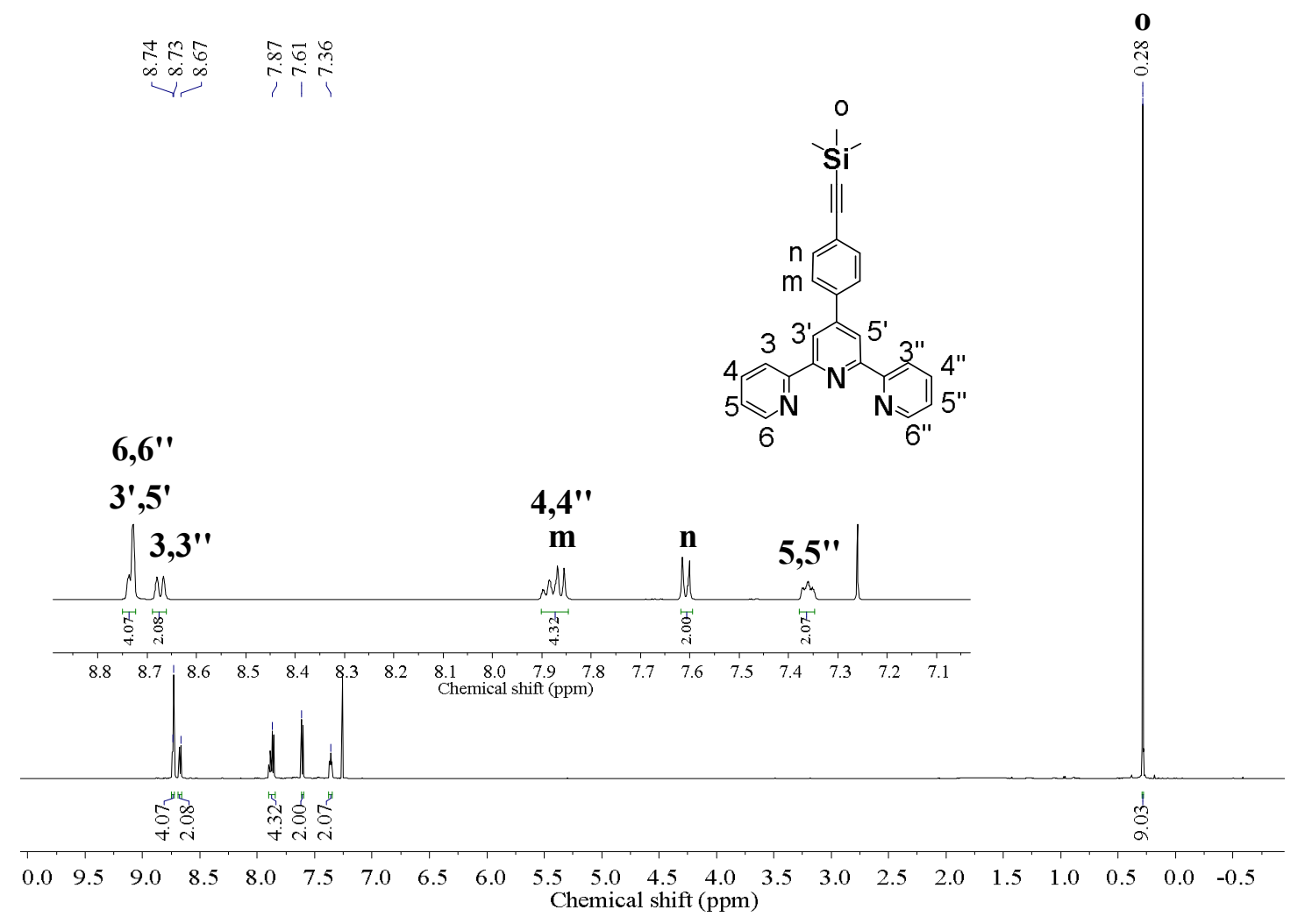

Figure S28. ${ }^{1} \mathrm{H} \mathrm{NMR}\left(600 \mathrm{MHz}, \mathrm{CDCl}_{3}\right)$ spectrum of compound 7. 


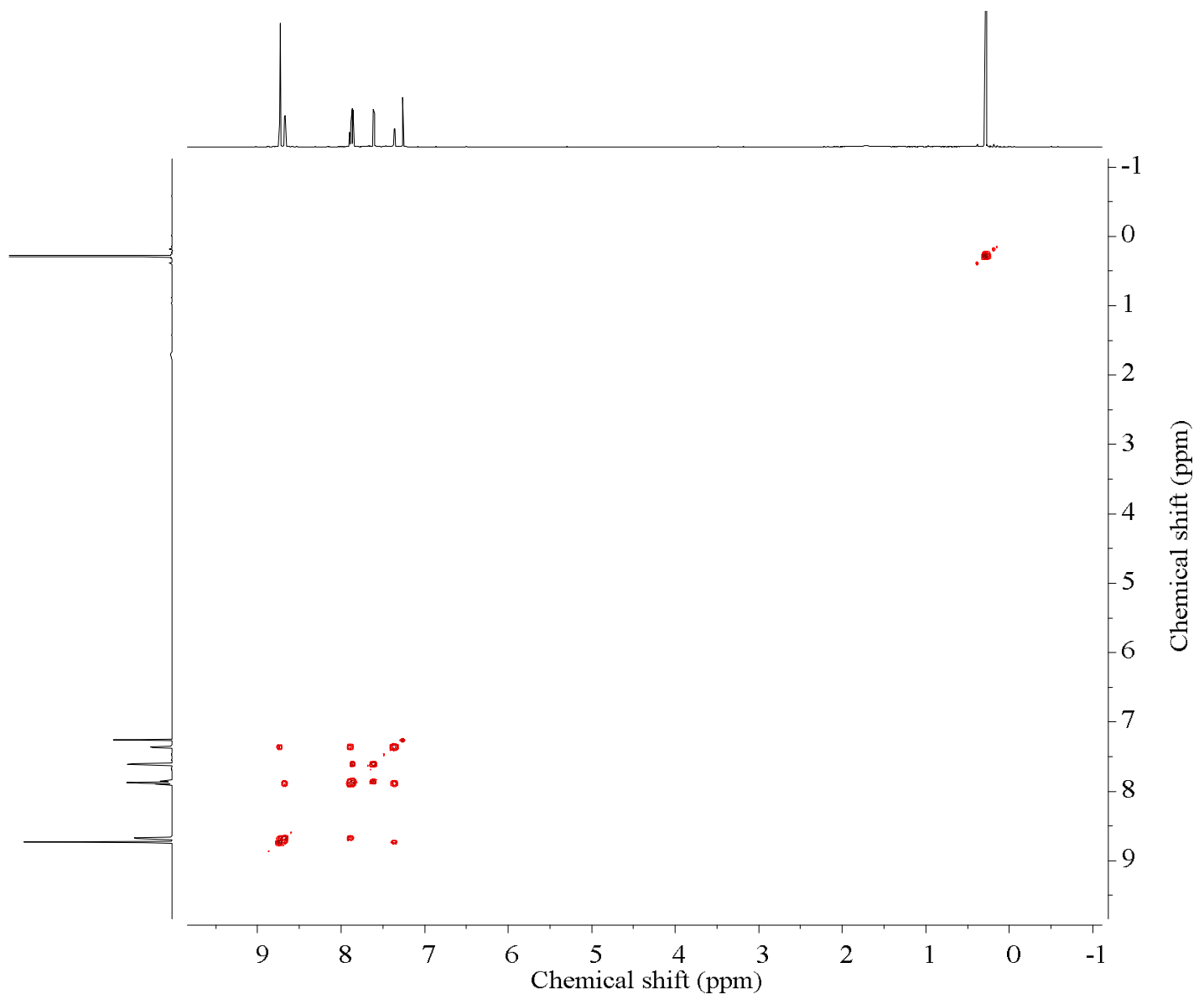

Figure S29. 2D COSY NMR (600 MHz, $\left.\mathrm{CDCl}_{3}\right)$ spectrum of compound 7.

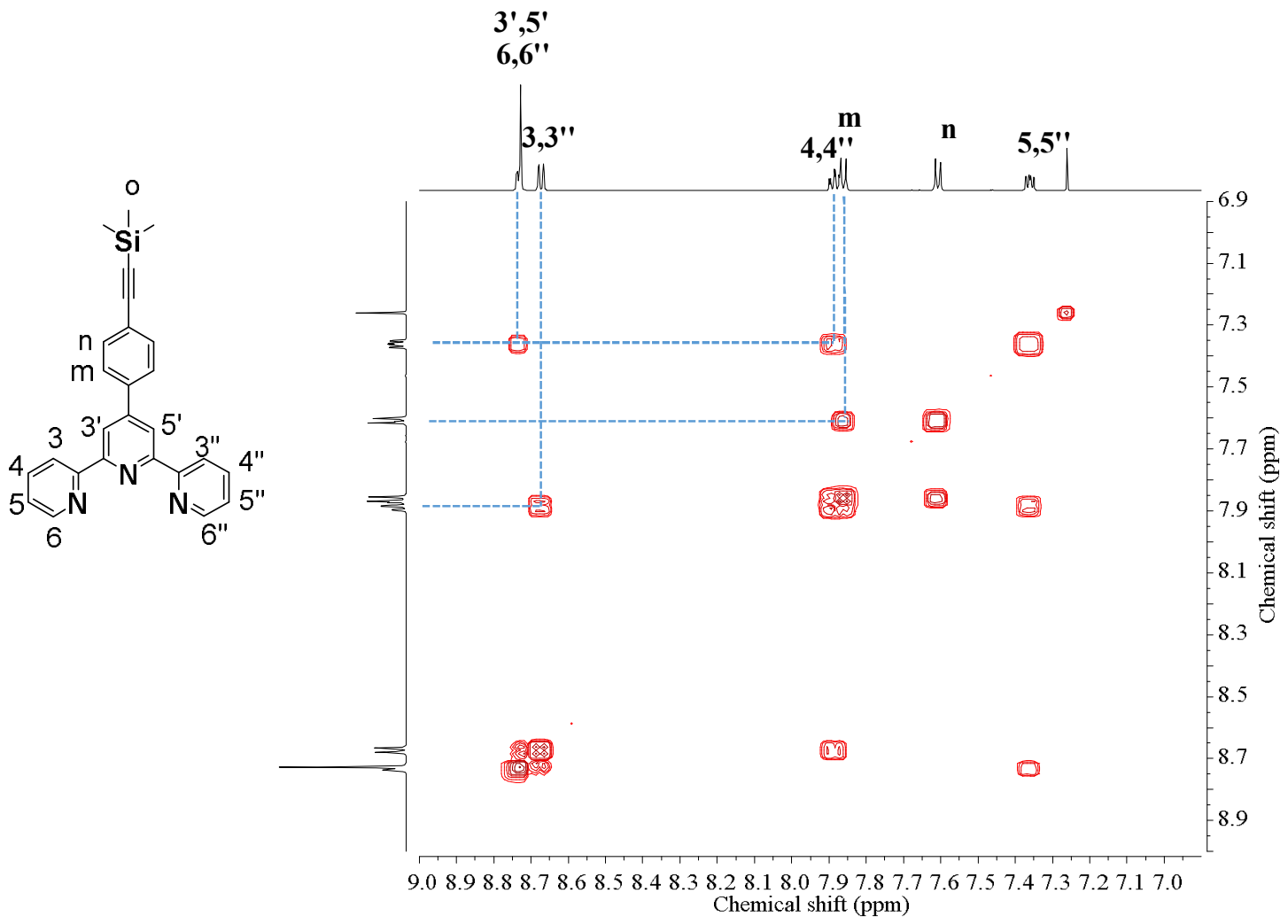

Figure S30. 2D COSY NMR (600 MHz, $\left.\mathrm{CDCl}_{3}\right)$ spectrum of compound 7 (aromatic region). 


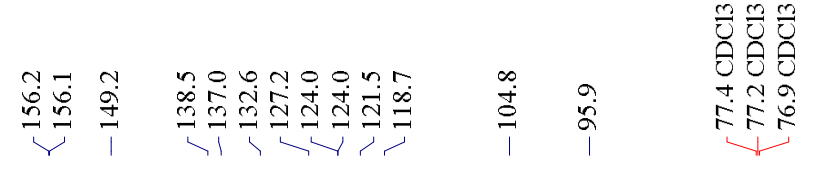

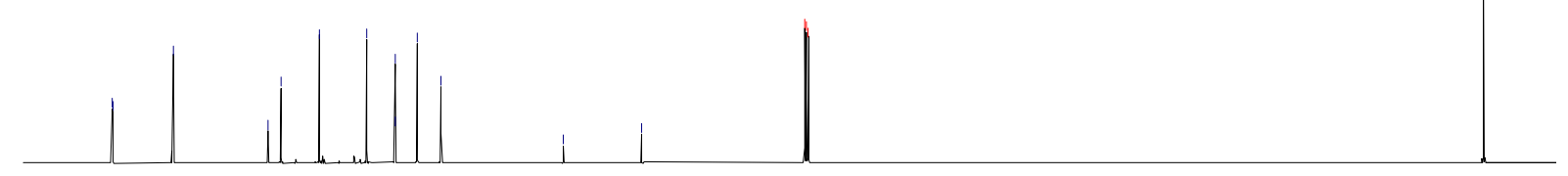

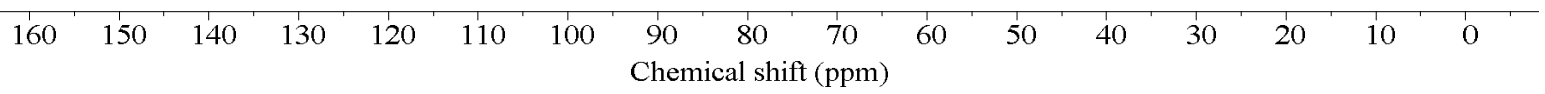

Figure S31. ${ }^{13} \mathrm{C}$ NMR $\left(150 \mathrm{MHz}, \mathrm{CDCl}_{3}\right)$ spectrum of compound 7.
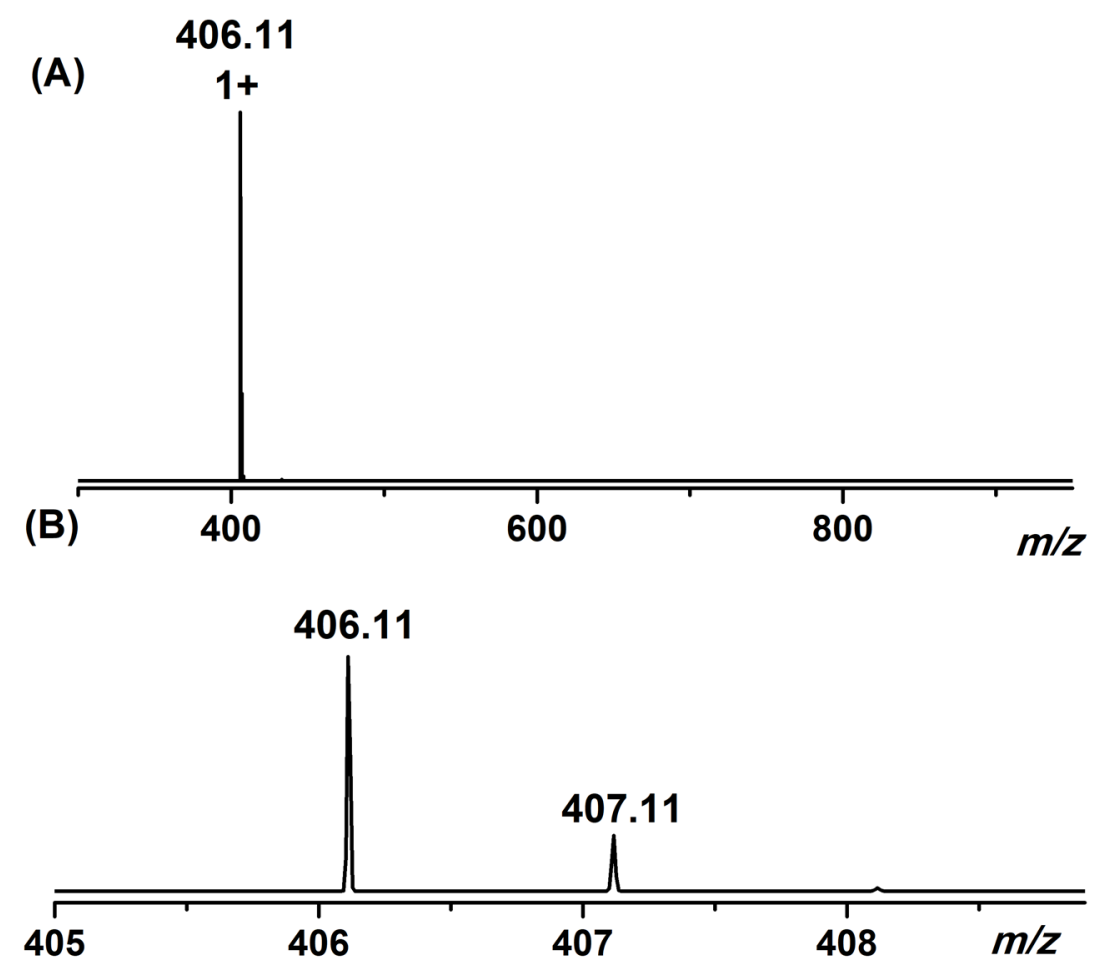

Figure S32. (A) ESI-MS spectrum of compound 7 in $\mathrm{CHCl}_{3} / \mathrm{CH}_{3} \mathrm{OH}(1 / 3)$ and (B) isotope pattern. 


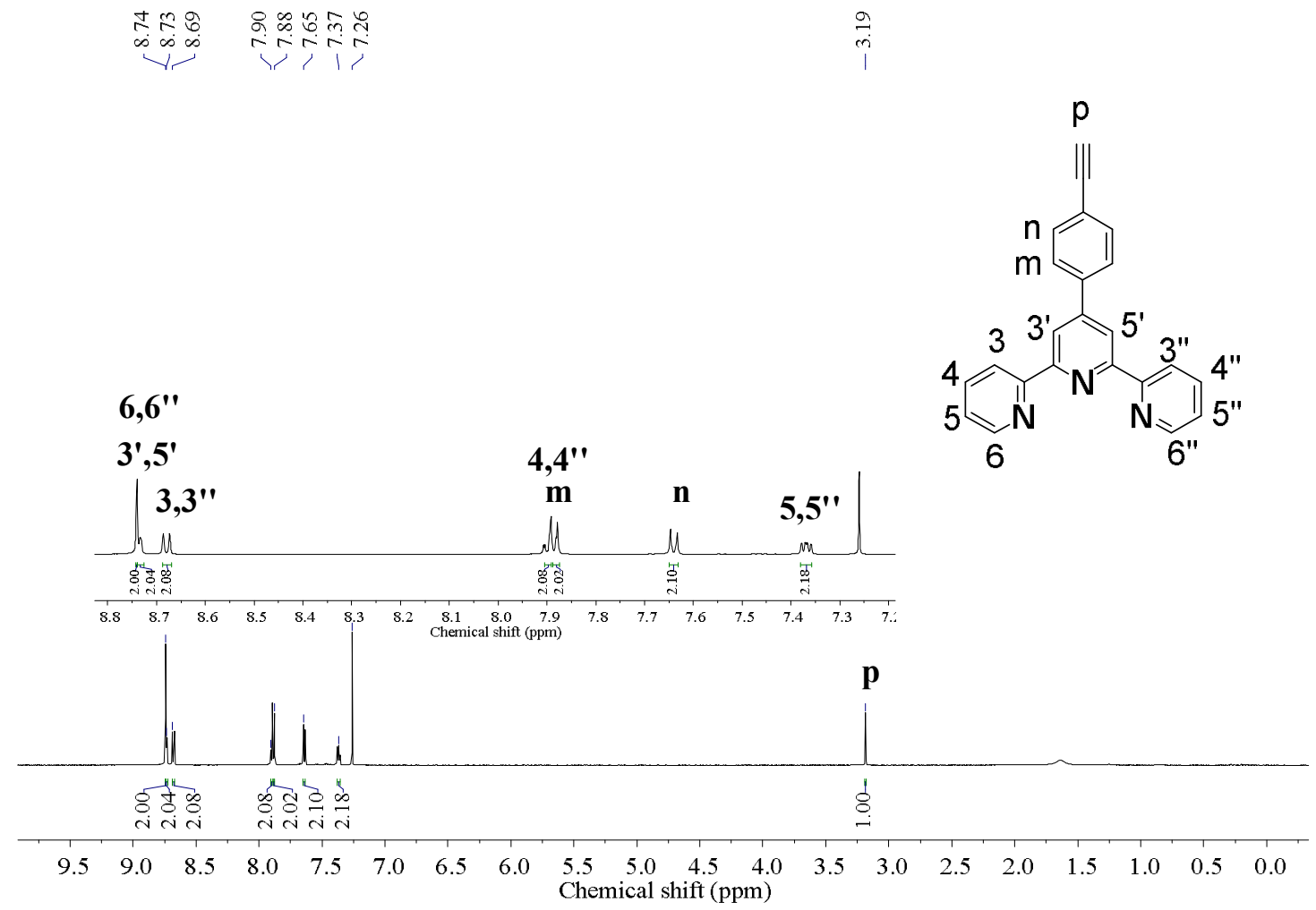

Figure S33. ${ }^{1} \mathrm{H}$ NMR $\left(600 \mathrm{MHz}, \mathrm{CDCl}_{3}\right)$ spectrum of compound 8 .

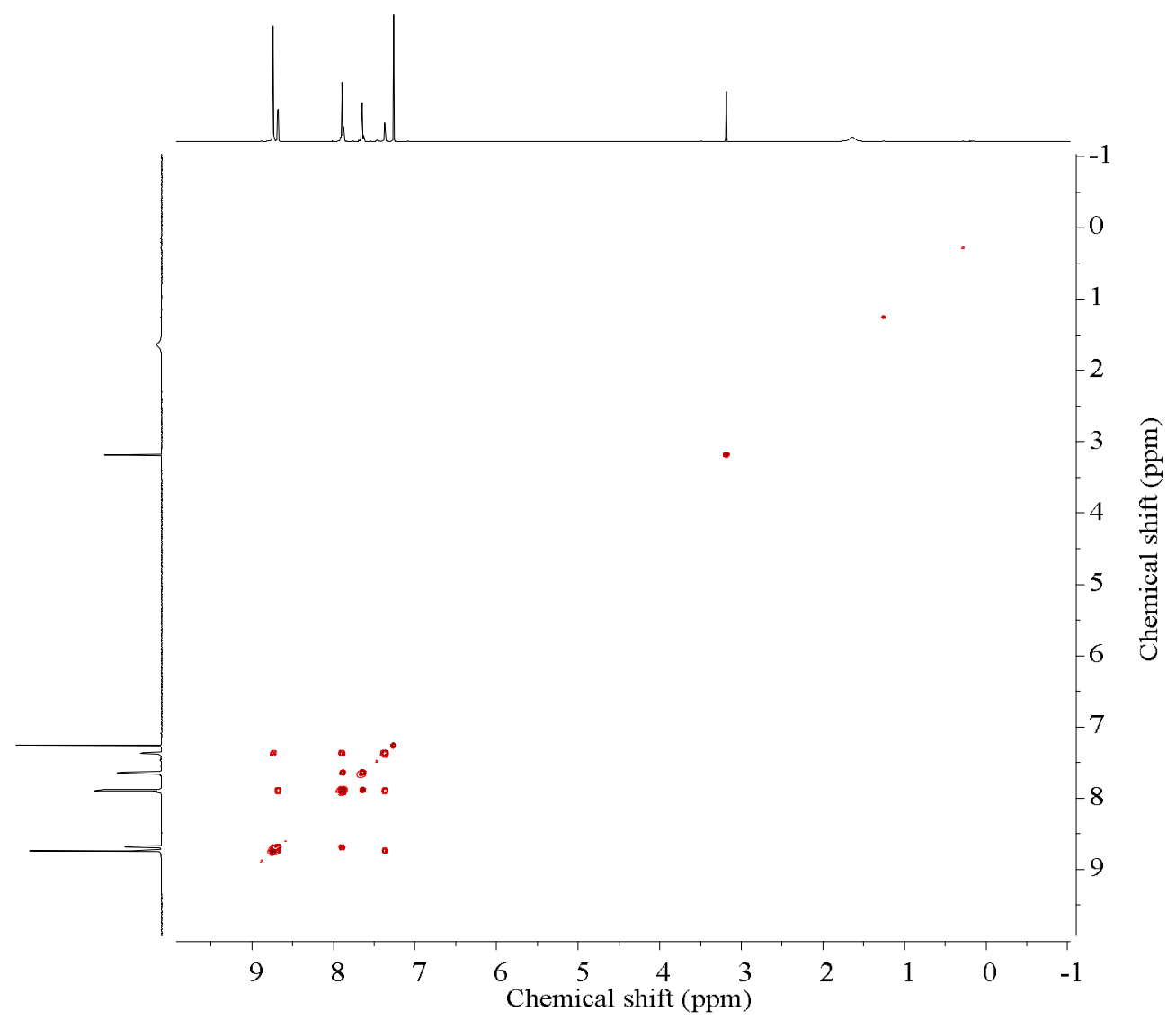

Figure S34. 2D COSY NMR (600 MHz, $\left.\mathrm{CDCl}_{3}\right)$ spectrum of compound 8 . 


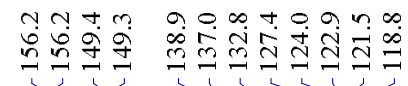
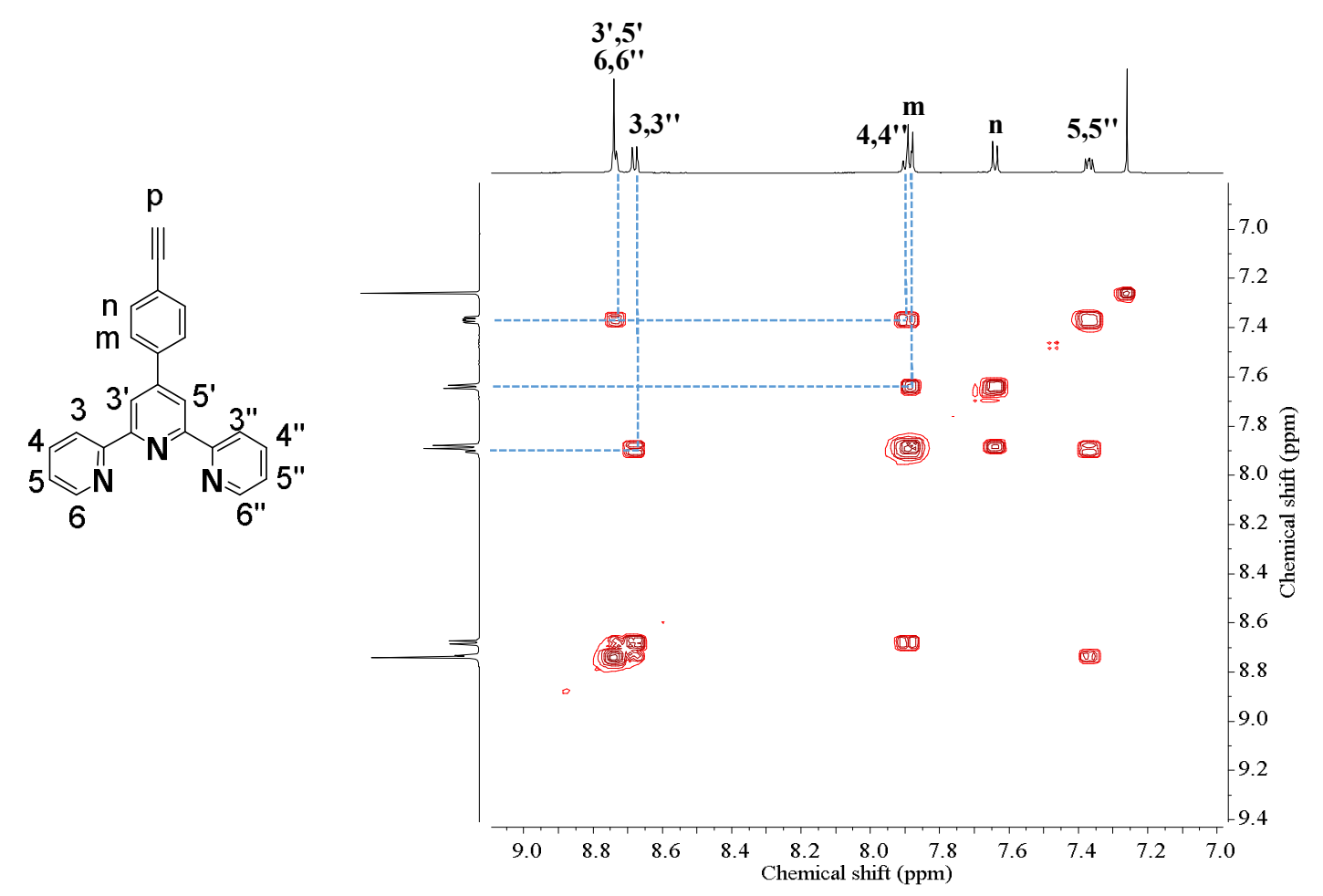

Figure S35. 2D COSY NMR ( $600 \mathrm{MHz}, \mathrm{CDCl}_{3}$ ) spectrum of compound 8 (aromatic region).

Higure S35. 2D COSY NMR (600

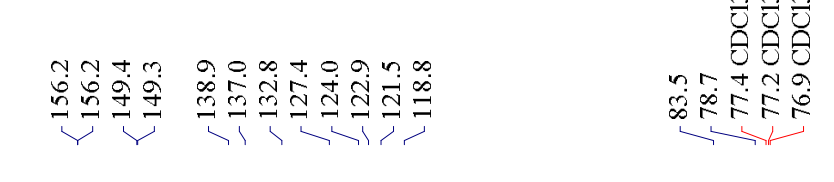


(A)

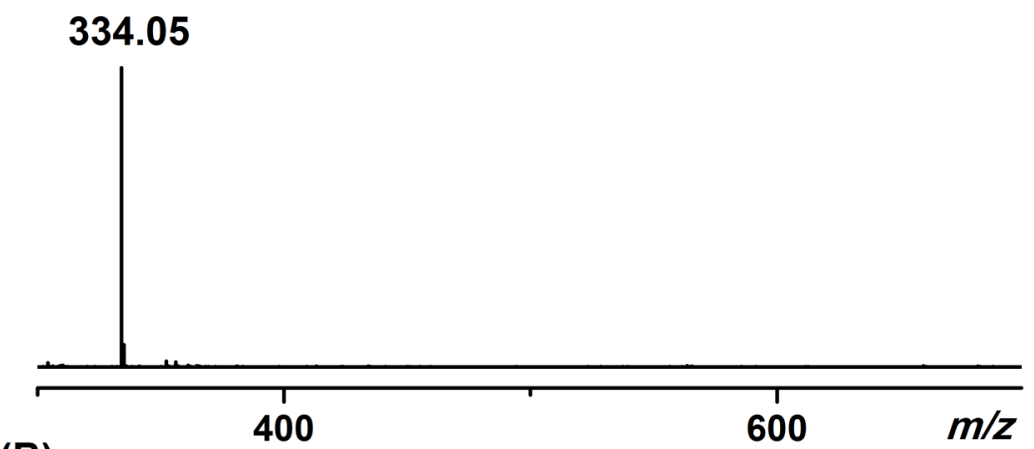

(B)

334.05

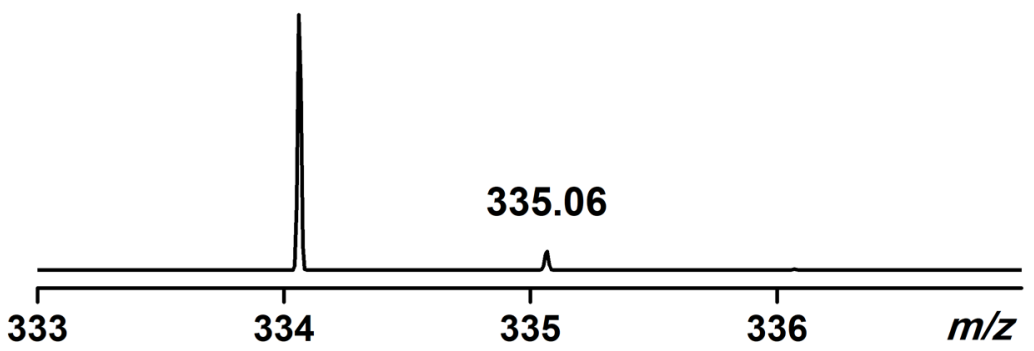

Figure S37. (A) ESI-MS spectrum of compound 8 in $\mathrm{CHCl}_{3} / \mathrm{CH}_{3} \mathrm{OH}(1 / 3)$ and (B) isotope pattern.

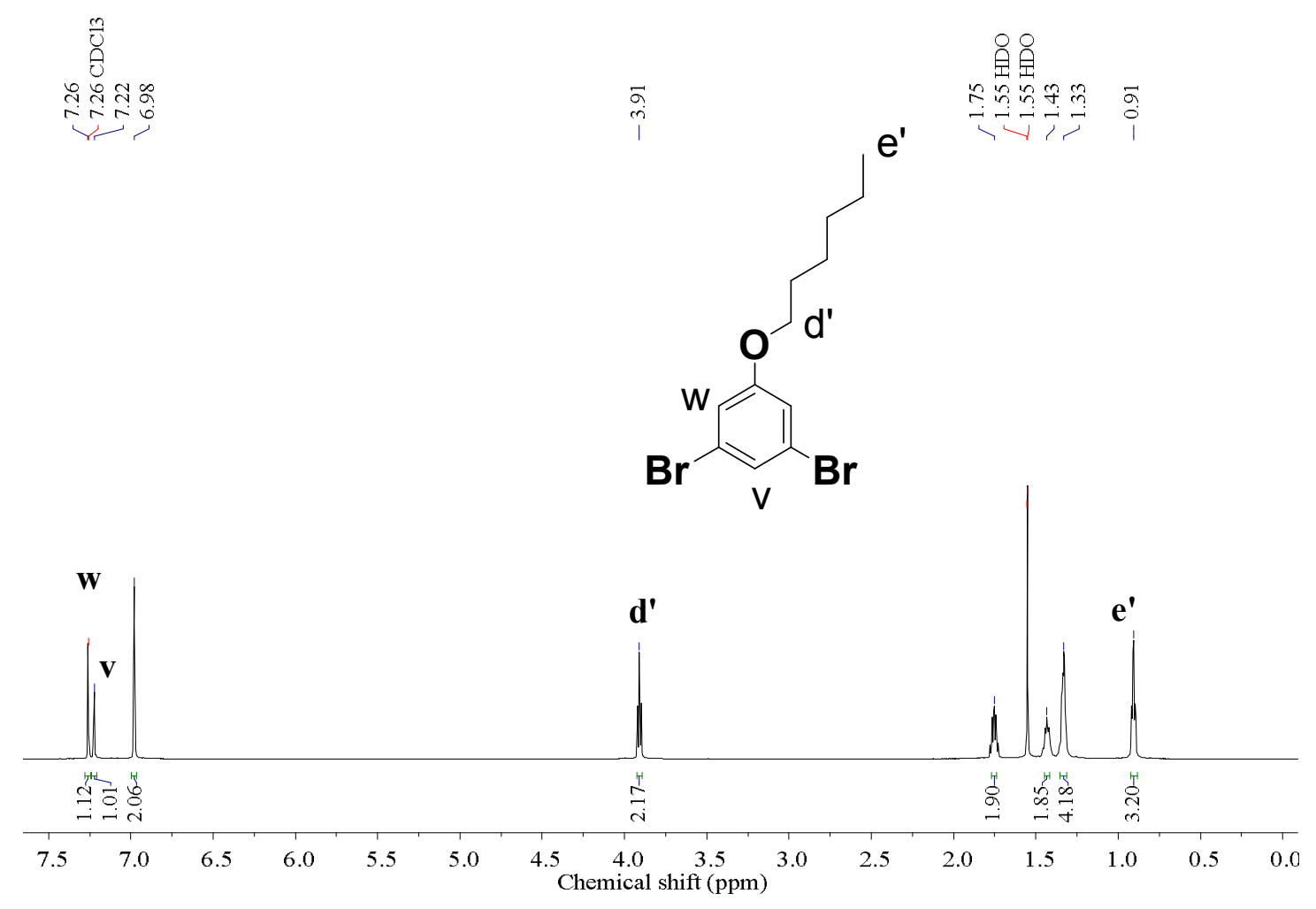

Figure S38. ${ }^{1} \mathrm{H}$ NMR $\left(600 \mathrm{MHz}, \mathrm{CDCl}_{3}\right)$ spectrum of compound 9. 


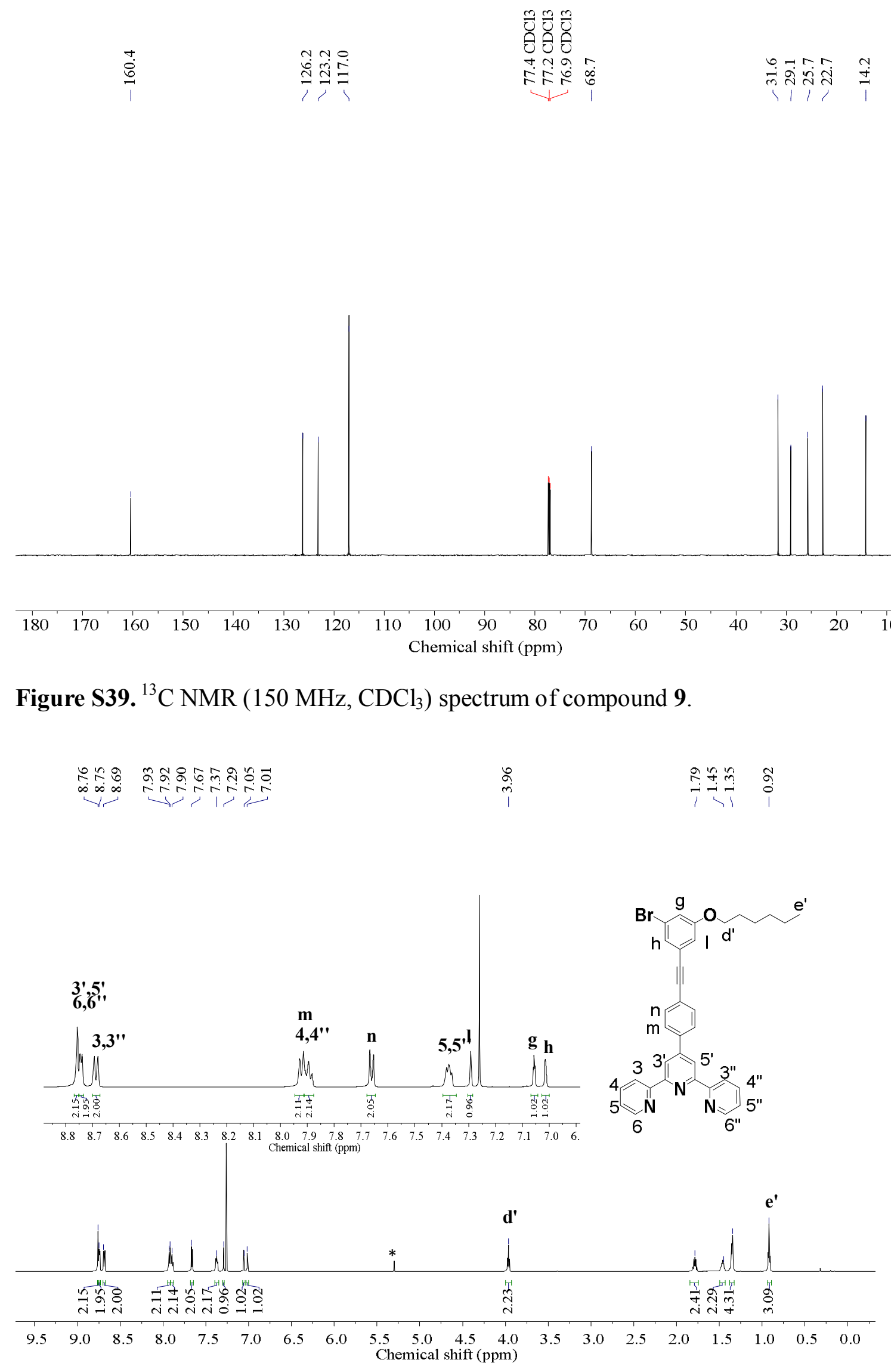

Figure S40. ${ }^{1} \mathrm{H} \mathrm{NMR}\left(600 \mathrm{MHz}, \mathrm{CDCl}_{3}\right)$ spectrum of compound $\mathbf{1 0}\left(*-\mathrm{CH}_{2} \mathrm{Cl}_{2}\right)$. 


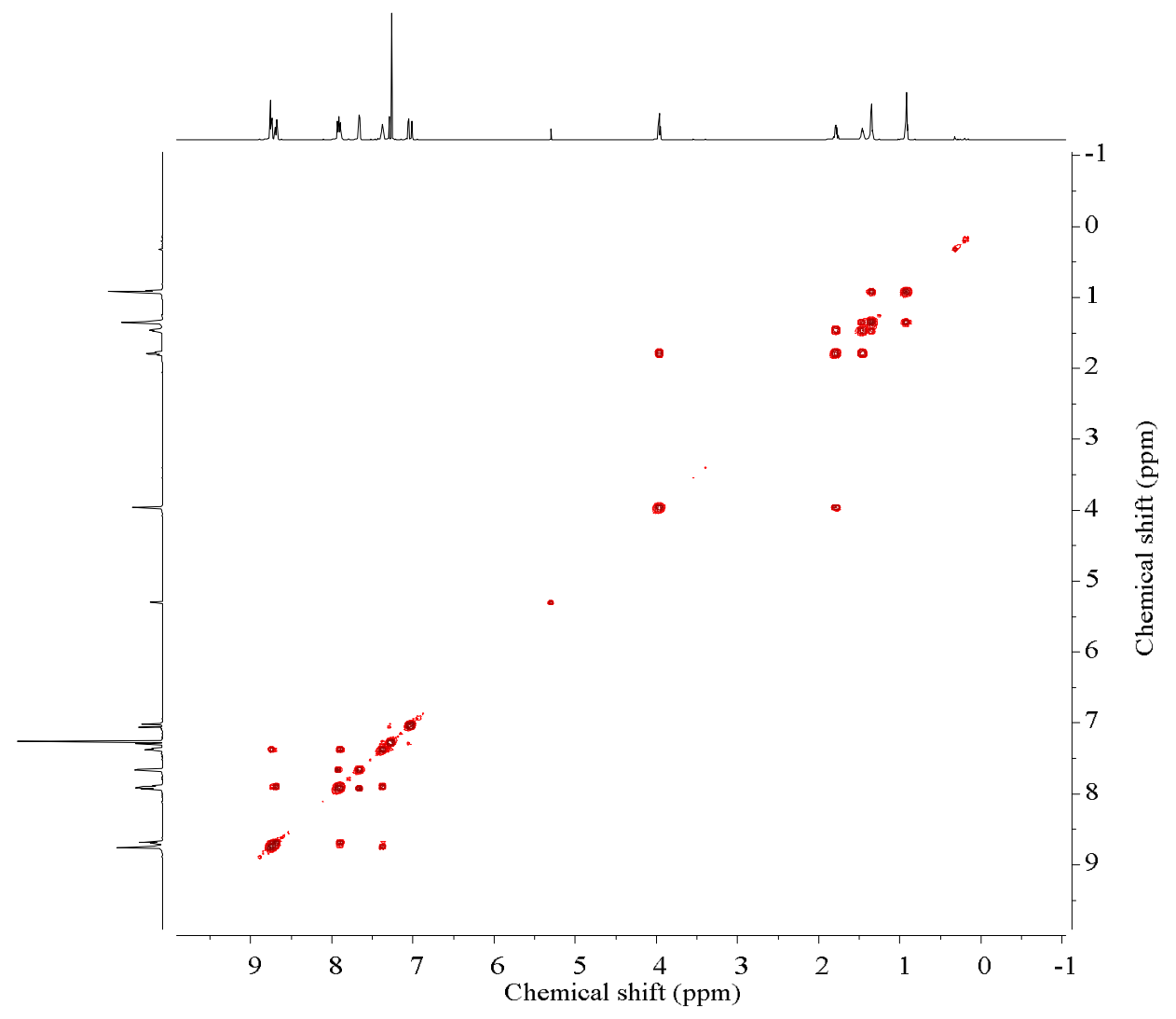

Figure S41. 2D COSY NMR (600 MHz, $\left.\mathrm{CDCl}_{3}\right)$ spectrum of compound 10.

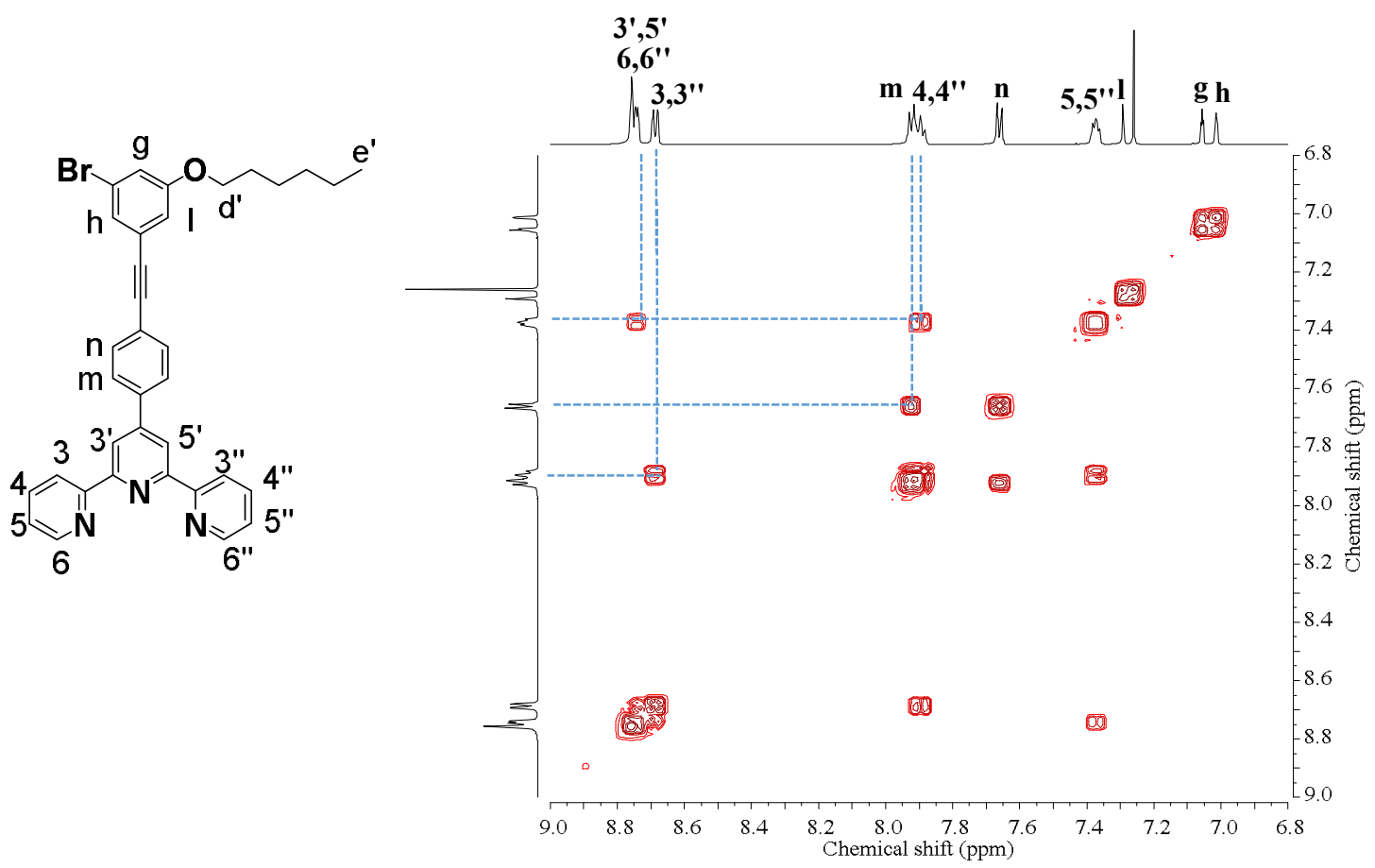

Figure S42. 2D COSY NMR (600 $\left.\mathrm{MHz}, \mathrm{CDCl}_{3}\right)$ spectrum of compound 10 (aromatic region). 


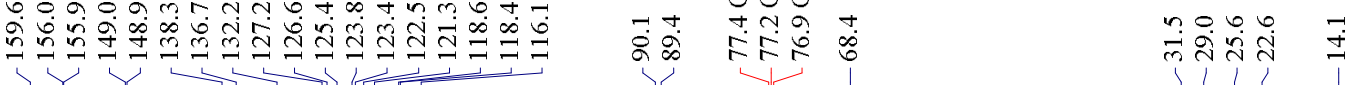

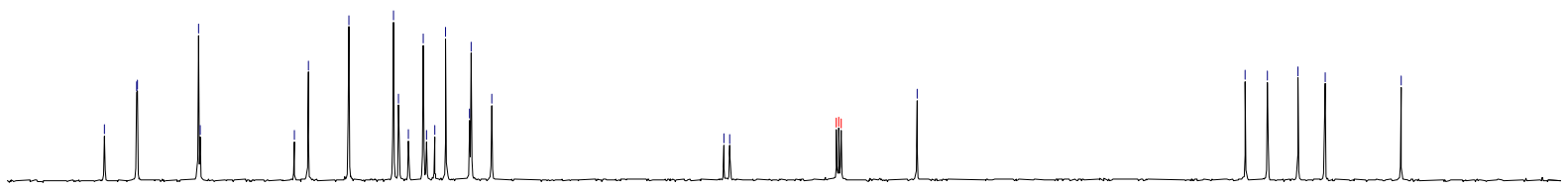

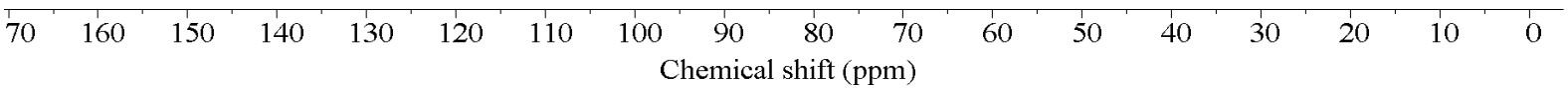

Figure S43. ${ }^{13} \mathrm{C}$ NMR $\left(150 \mathrm{MHz}, \mathrm{CDCl}_{3}\right)$ spectrum of compound $\mathbf{1 0}$.

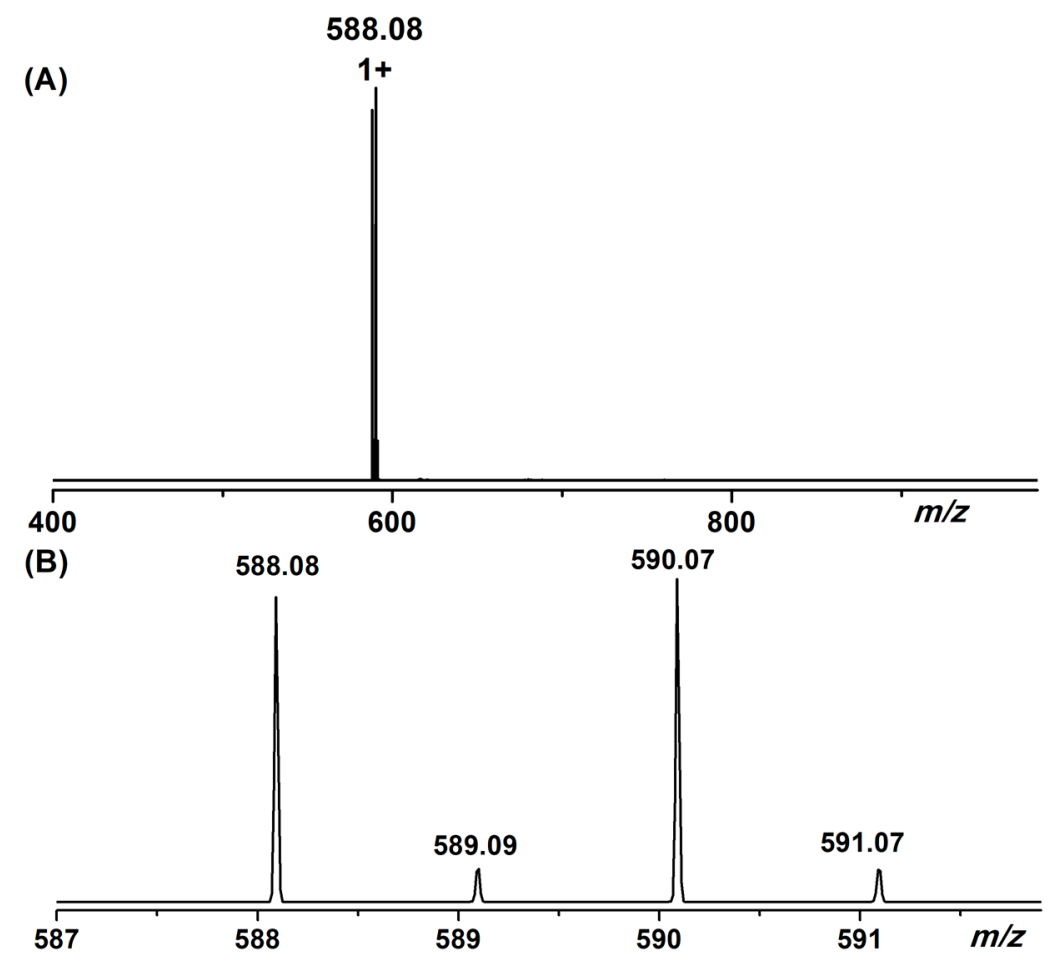

Figure S44. (A) ESI-MS spectrum of compound 10 in $\mathrm{CHCl}_{3} / \mathrm{CH}_{3} \mathrm{OH}(1 / 3)$ and (B) isotope pattern. 


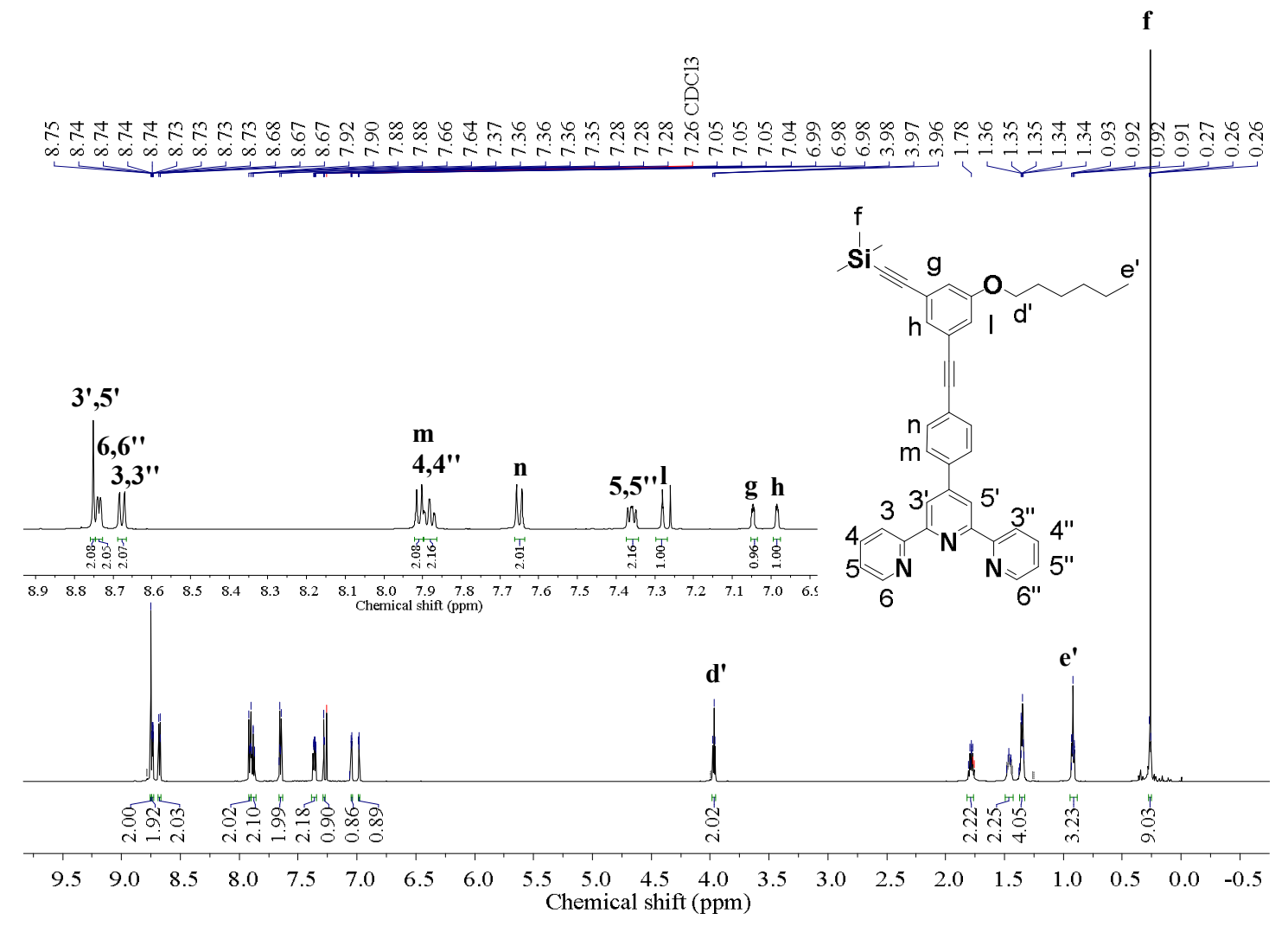

Figure S45. ${ }^{1} \mathrm{H}$ NMR $\left(600 \mathrm{MHz}, \mathrm{CDCl}_{3}\right)$ spectrum of compound 11.

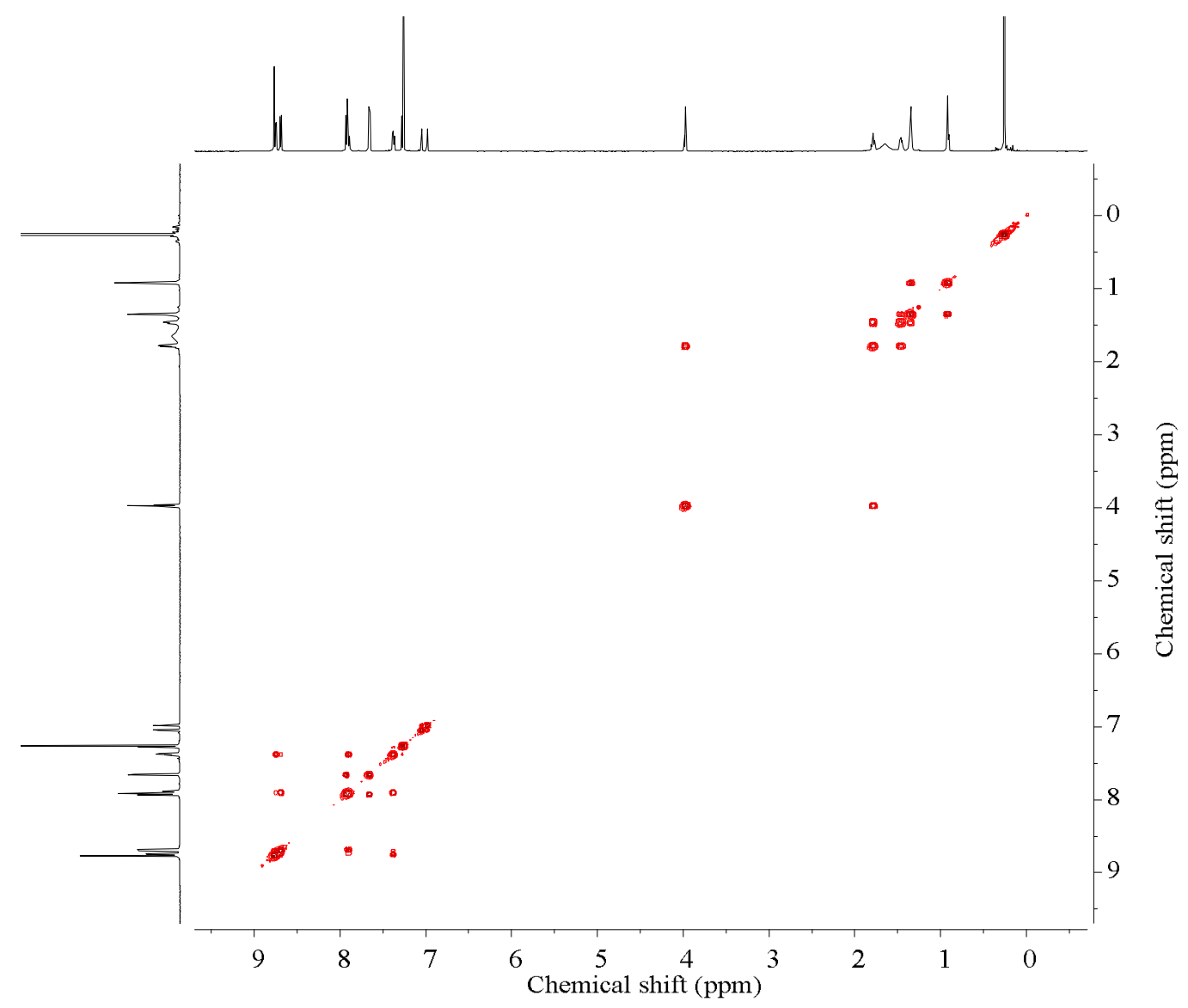

Figure S46. 2D COSY NMR (600 MHz, $\left.\mathrm{CDCl}_{3}\right)$ spectrum of compound 11. 


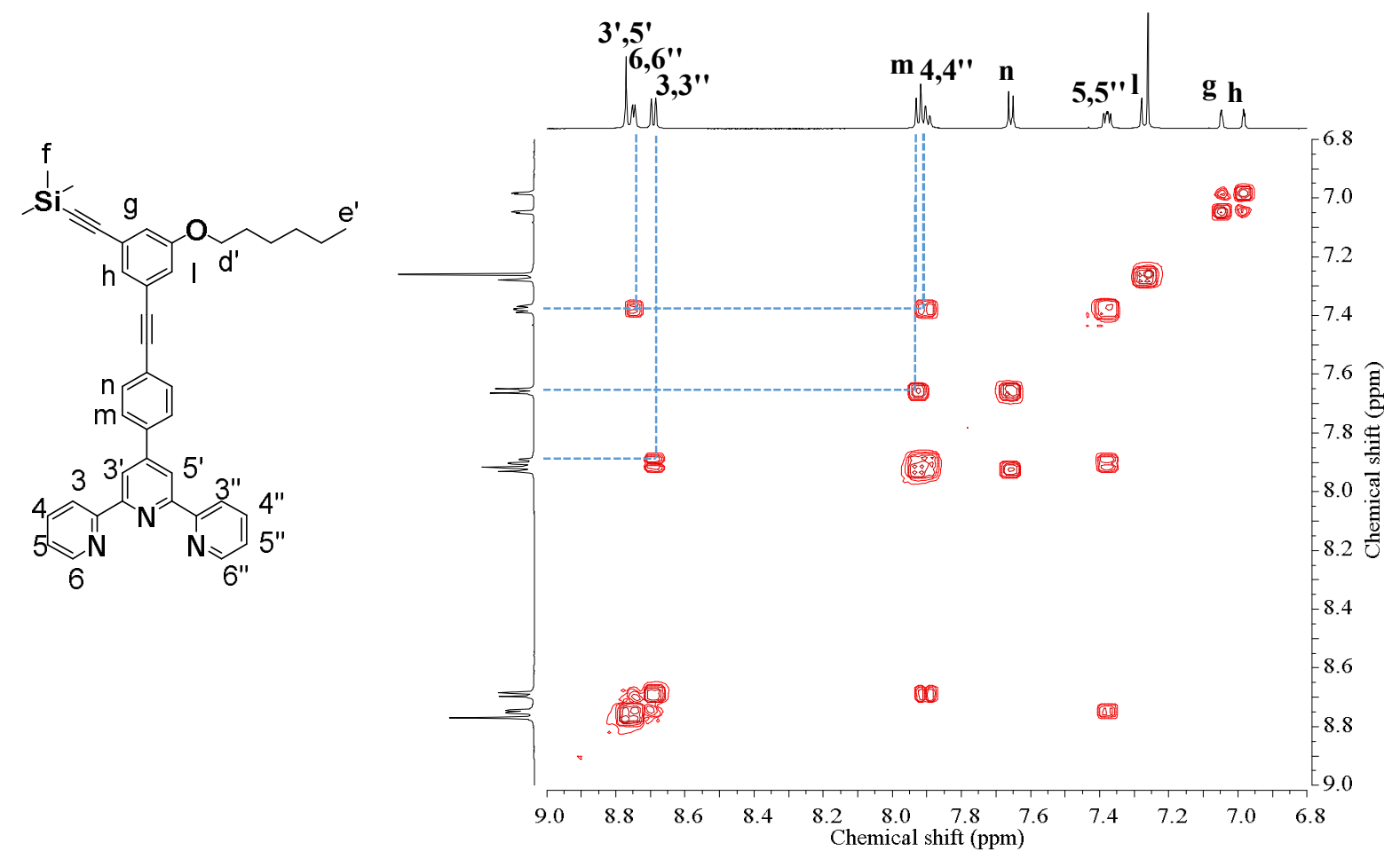

Figure S47. 2D COSY NMR $\left(600 \mathrm{MHz}, \mathrm{CDCl}_{3}\right)$ spectrum of compound 11 (aromatic region).
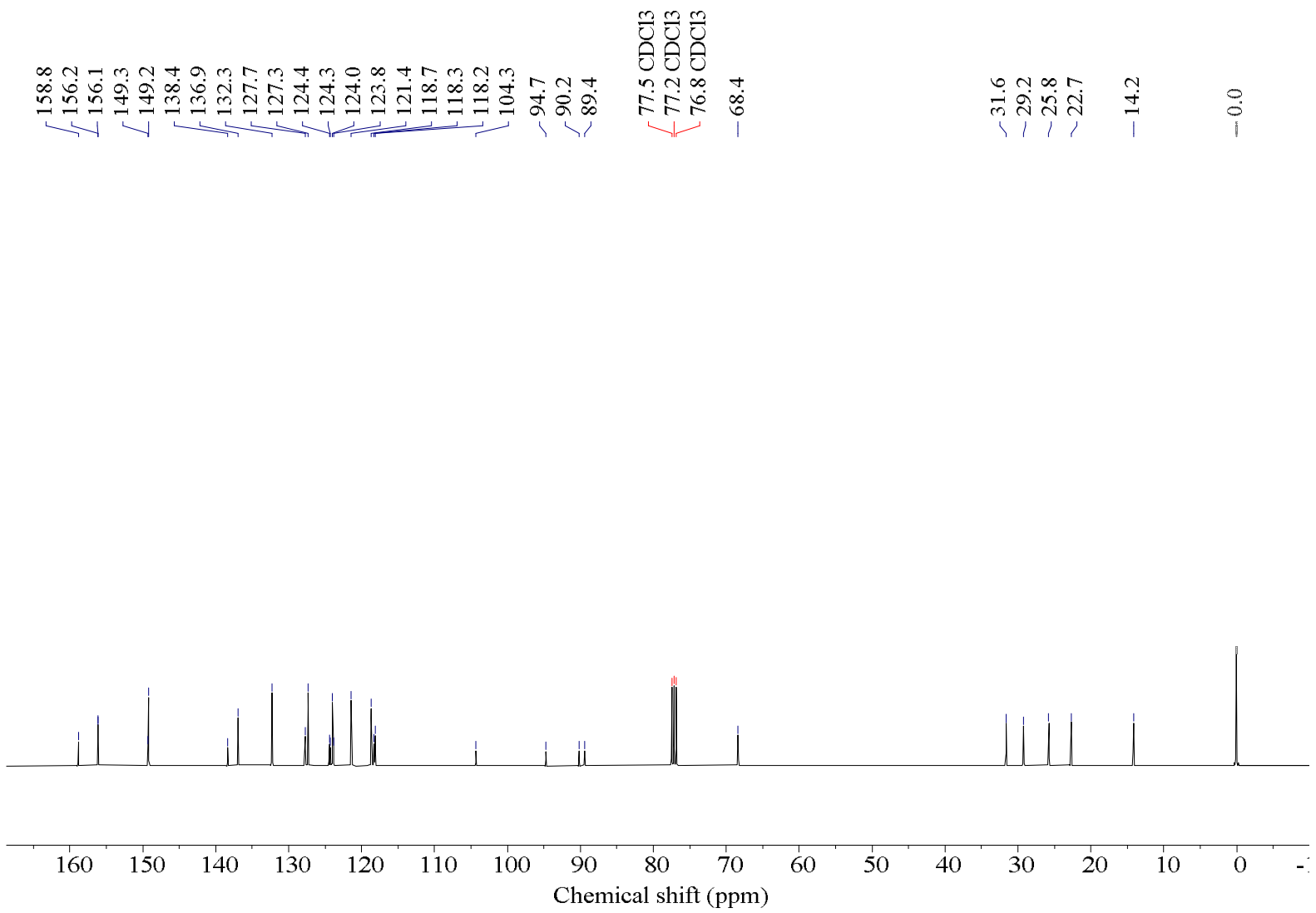

Figure S48. ${ }^{13} \mathrm{C}$ NMR $\left(100 \mathrm{MHz}, \mathrm{CDCl}_{3}\right)$ spectrum of compound 11. 


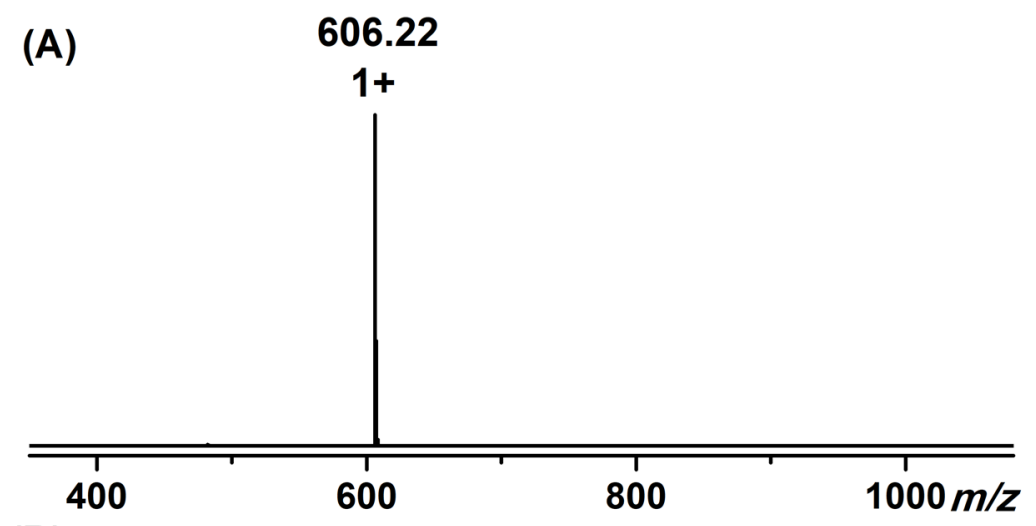

(B)

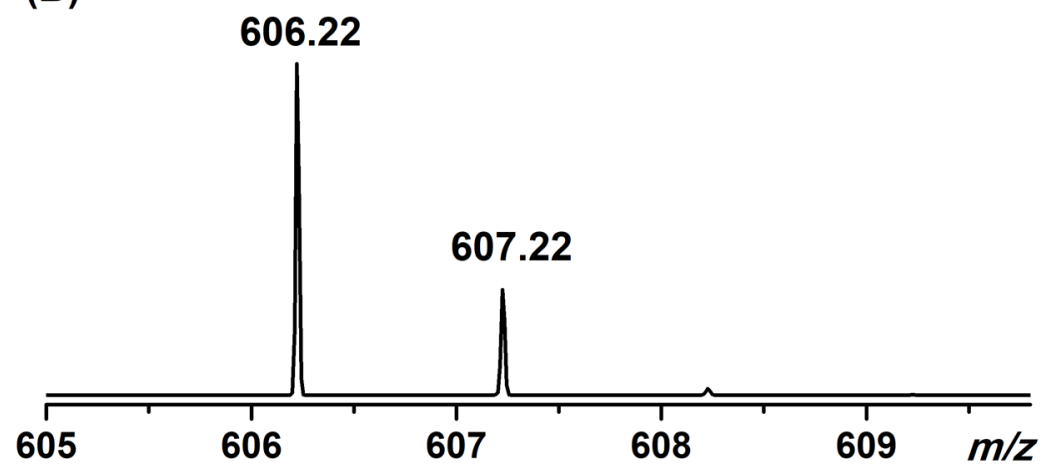

Figure S49. (A) ESI-MS spectrum of compound 11 in $\mathrm{CHCl}_{3} / \mathrm{CH}_{3} \mathrm{OH}(1 / 3)$ and (B) isotope pattern.
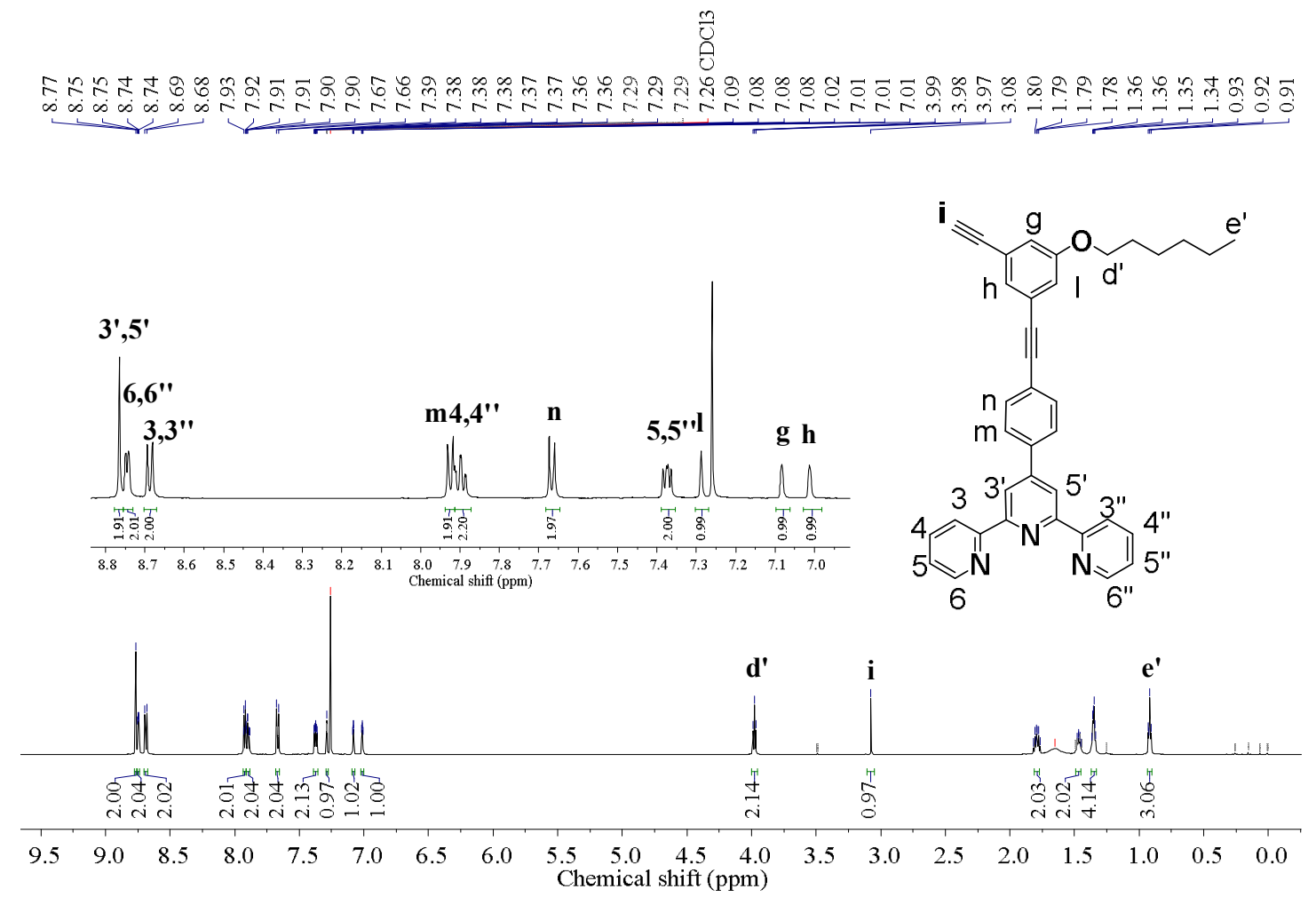

Figure S50. ${ }^{1} \mathrm{H}$ NMR $\left(600 \mathrm{MHz}, \mathrm{CDCl}_{3}\right)$ spectrum of compound 12. 


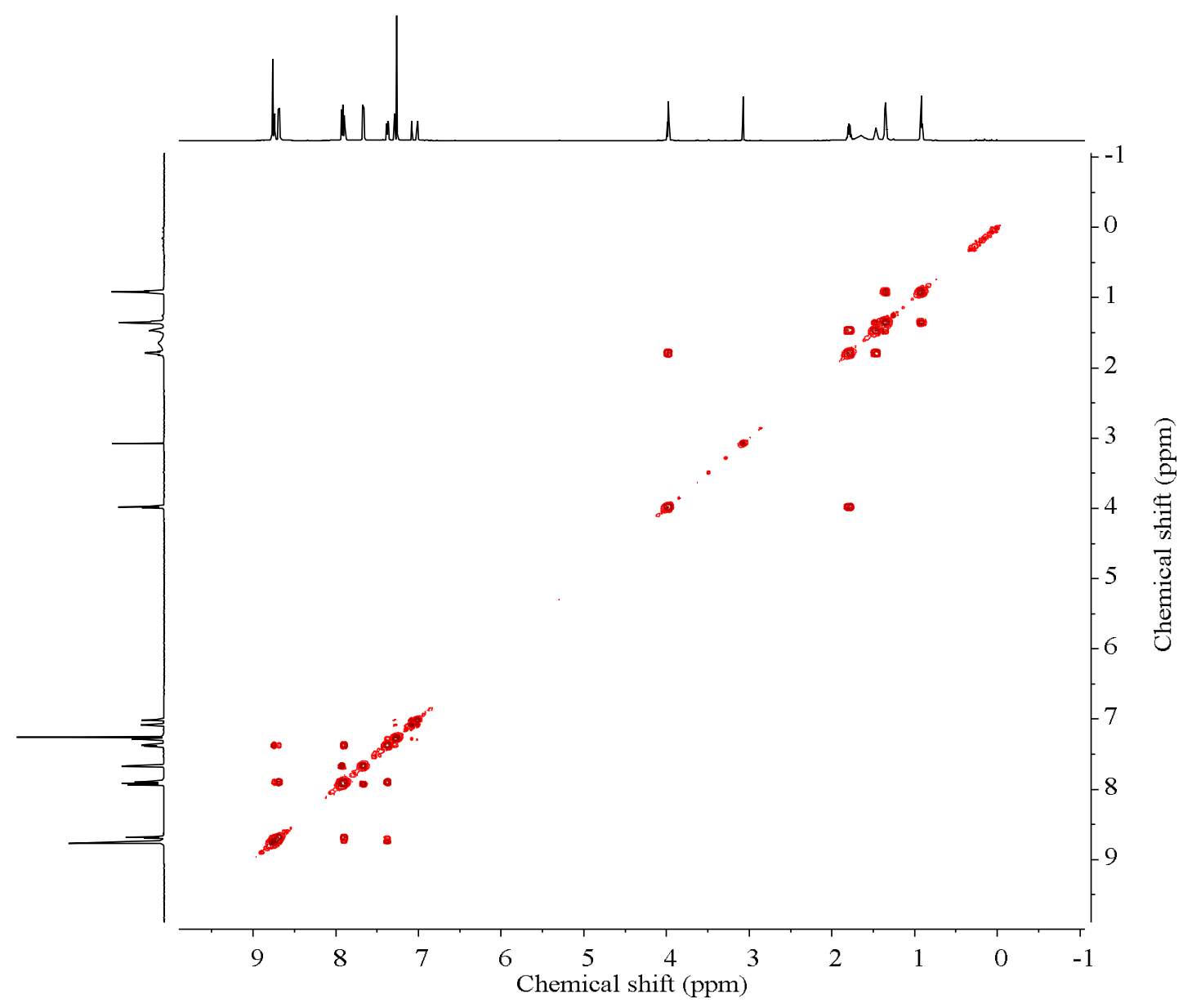

Figure S51. 2D COSY NMR (600 MHz, $\left.\mathrm{CDCl}_{3}\right)$ spectrum of compound 12.

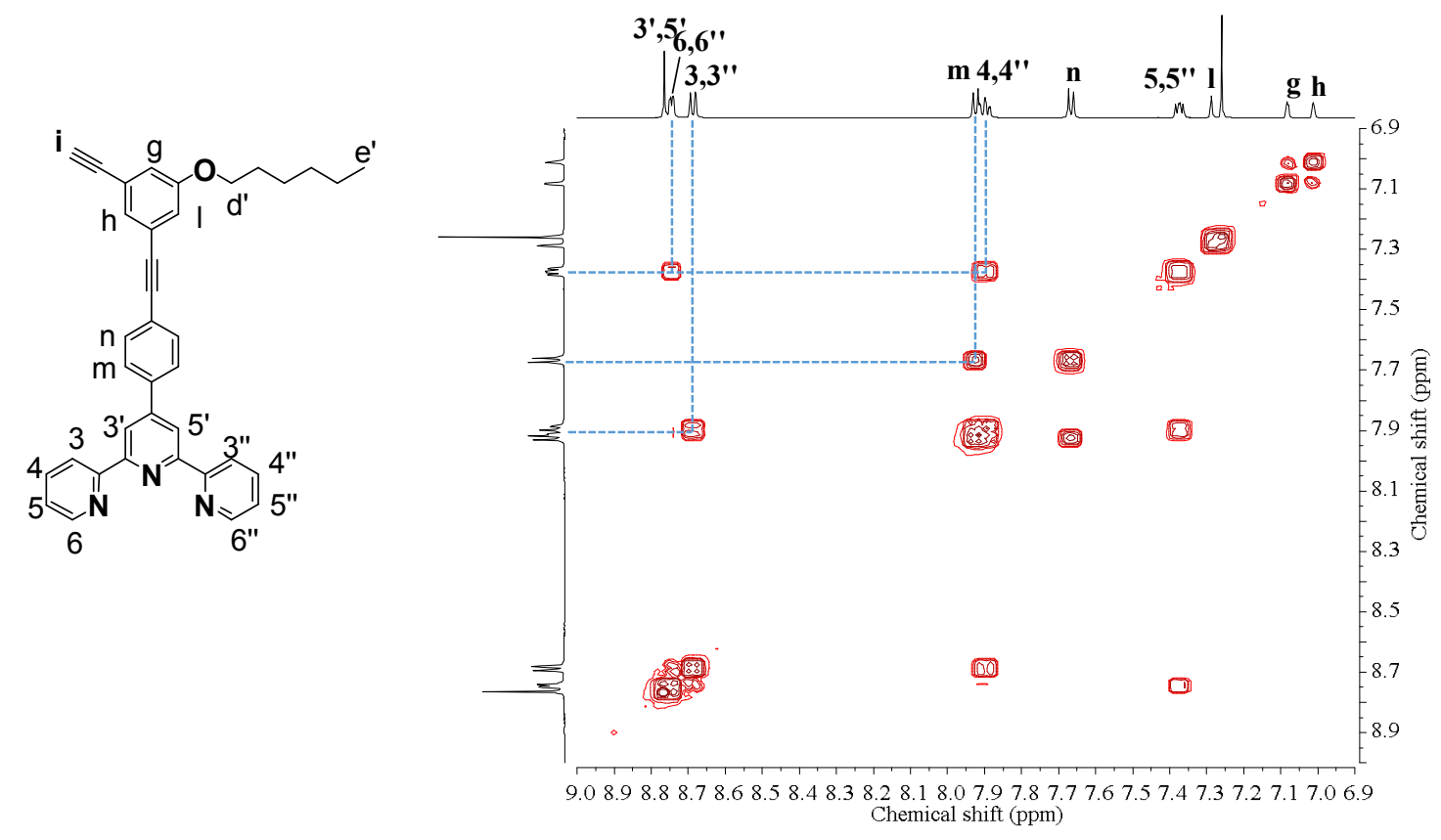

Figure S52. 2D COSY NMR (600 $\left.\mathrm{MHz}, \mathrm{CDCl}_{3}\right)$ spectrum of compound $\mathbf{1 2}$ (aromatic region). 


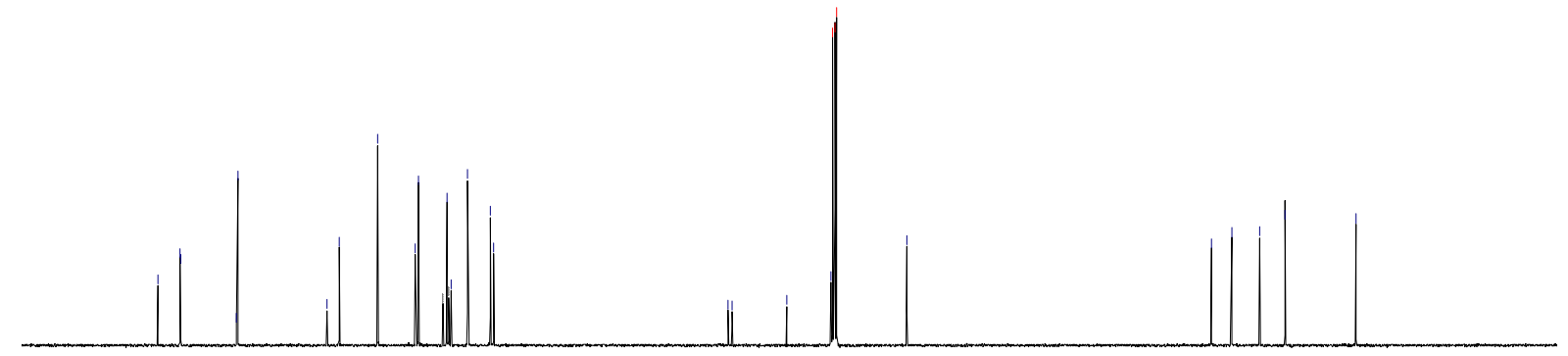

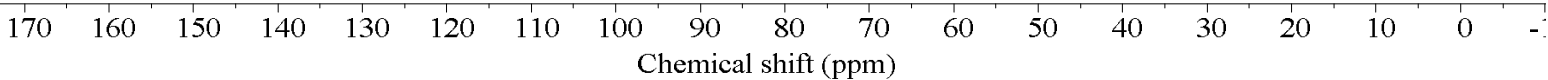

Figure S53. ${ }^{13} \mathrm{C}$ NMR $\left(150 \mathrm{MHz}, \mathrm{CDCl}_{3}\right)$ spectrum of compound 12.

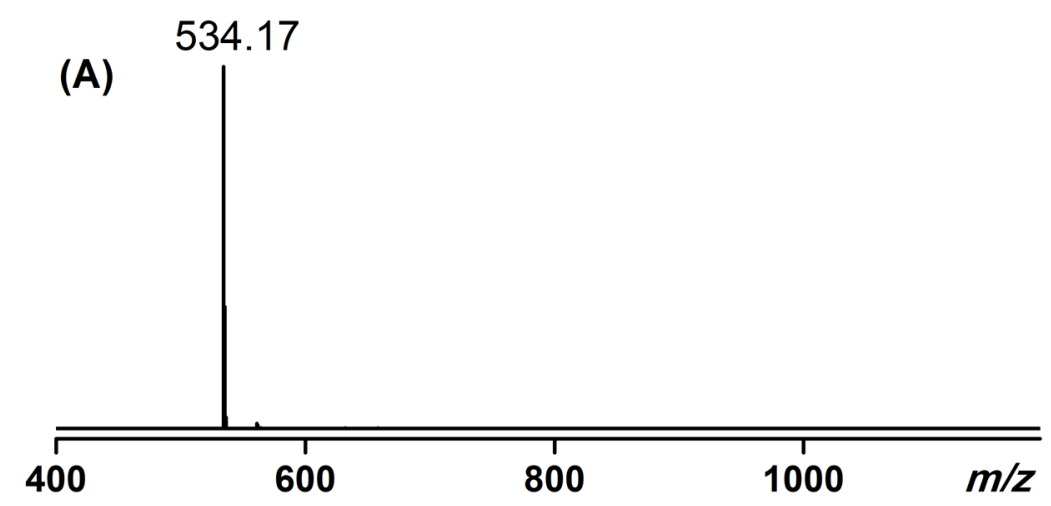

(B)

534.17

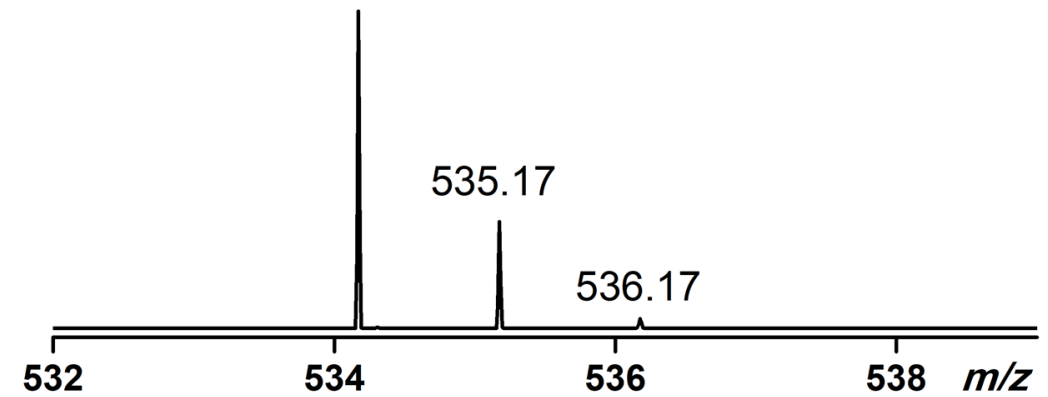

Figure S54. (A) ESI-MS spectrum of compound 12 in $\mathrm{CHCl}_{3} / \mathrm{CH}_{3} \mathrm{OH}(1 / 3)$ and (B) isotope pattern. 

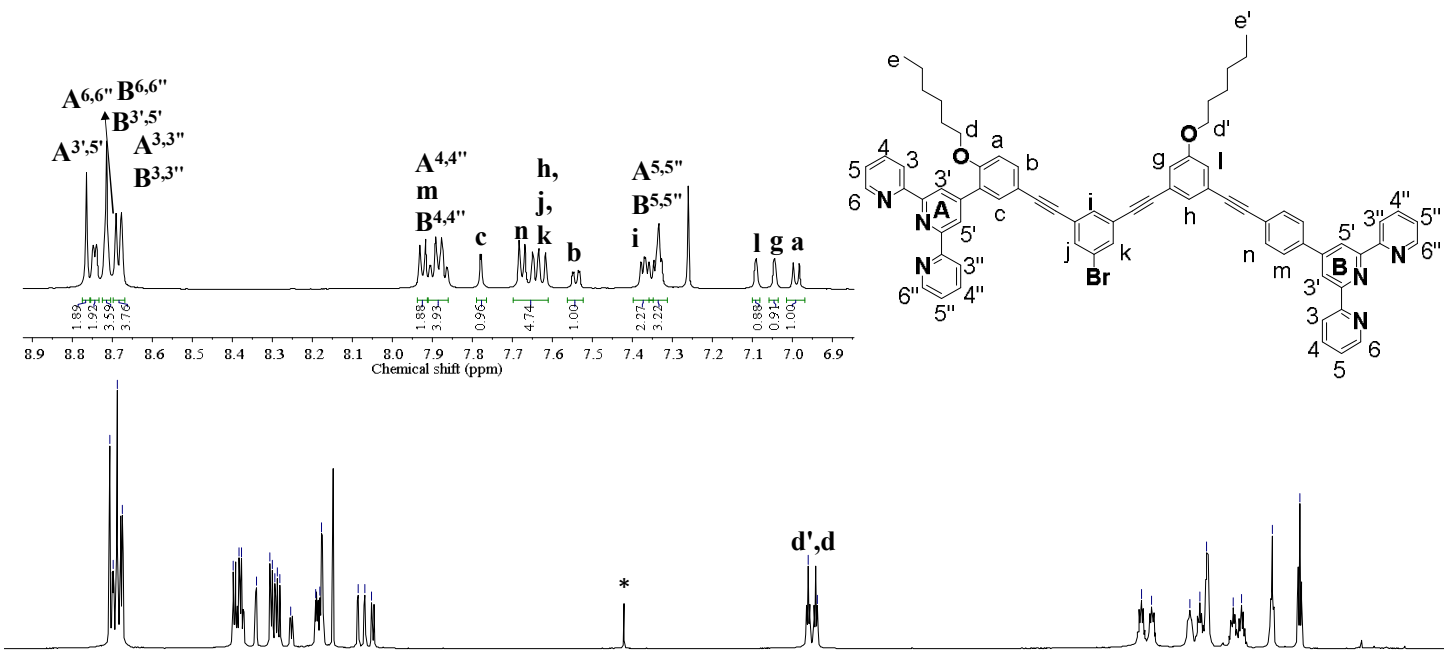

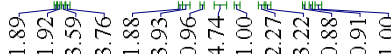

$\overbrace{1}^{n+1} 8$

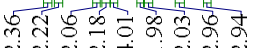
$\begin{array}{llllll}9.0 & 8.5 & 8.0 & 75 & 70 & 6.5\end{array}$

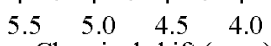

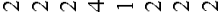

Figure S55. ${ }^{1} \mathrm{H} \mathrm{NMR}\left(600 \mathrm{MHz}, \mathrm{CDCl}_{3}\right)$ spectrum of compound $\mathbf{1 3}\left(*-\mathrm{CH}_{2} \mathrm{Cl}_{2}\right)$.

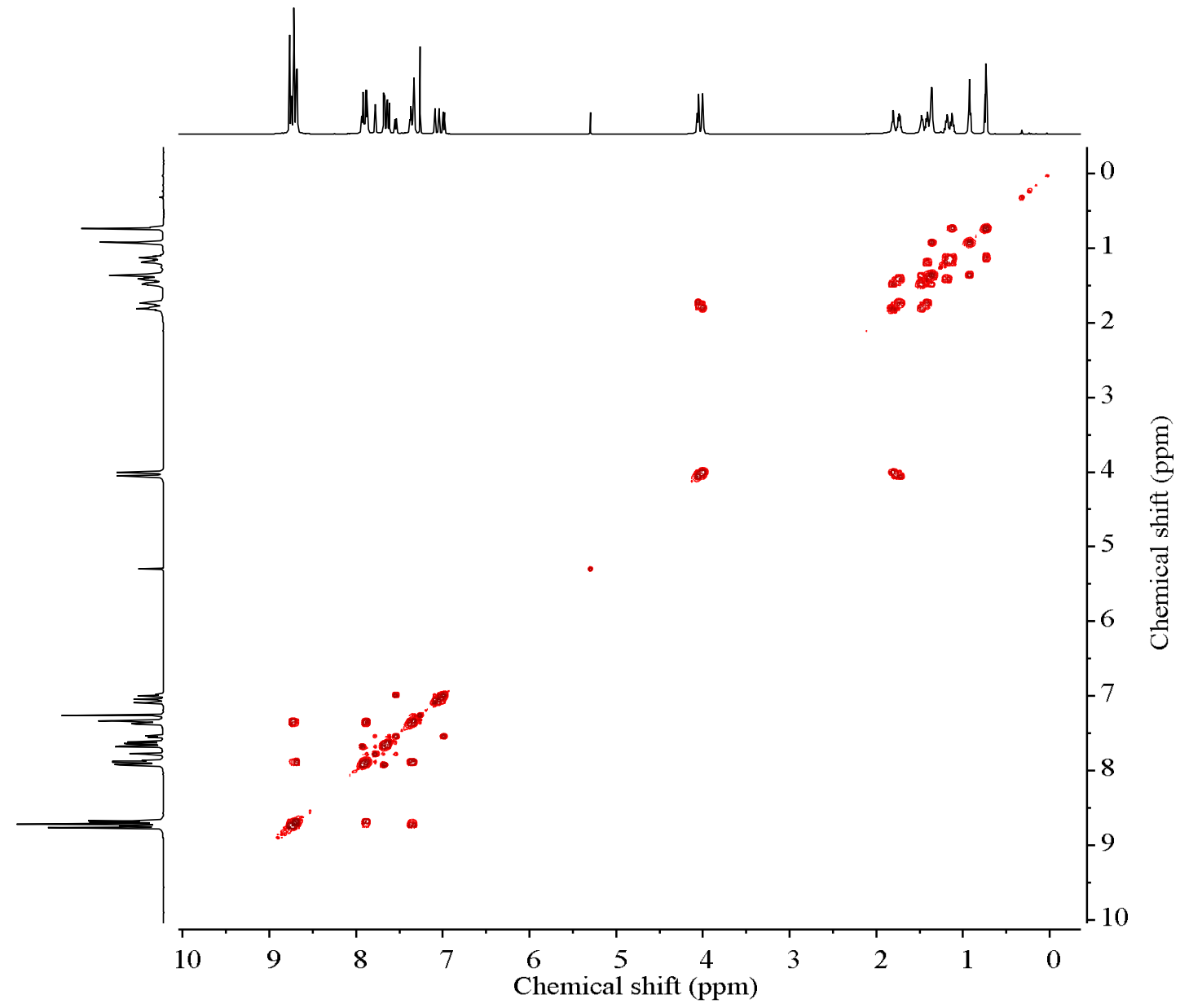

Figure S56. 2D COSY NMR (600 MHz, $\left.\mathrm{CDCl}_{3}\right)$ spectrum of compound 13. 

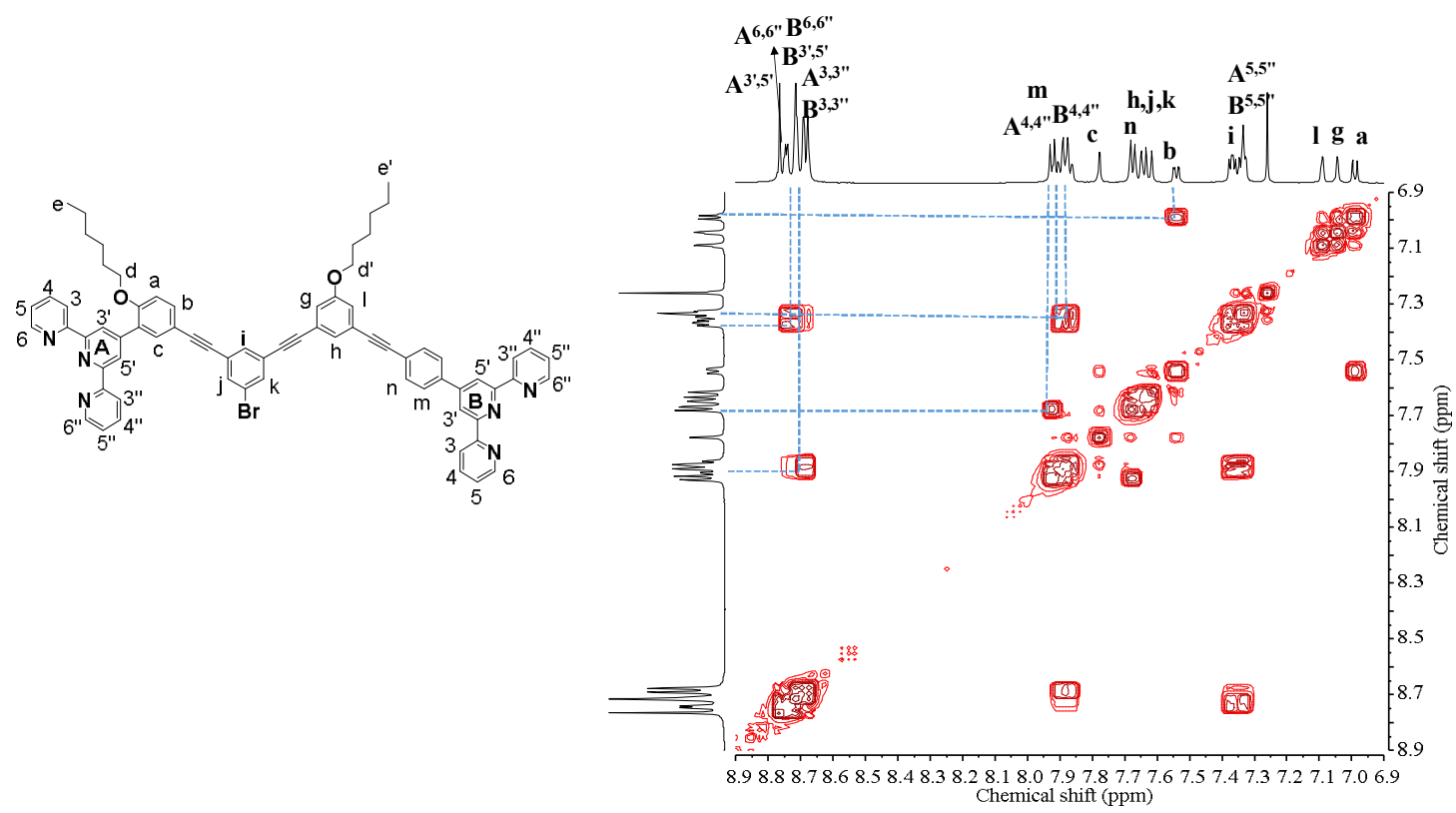

Figure S57. 2D COSY NMR (600 $\left.\mathrm{MHz}, \mathrm{CDCl}_{3}\right)$ spectrum of compound $\mathbf{1 3}$ (aromatic region).

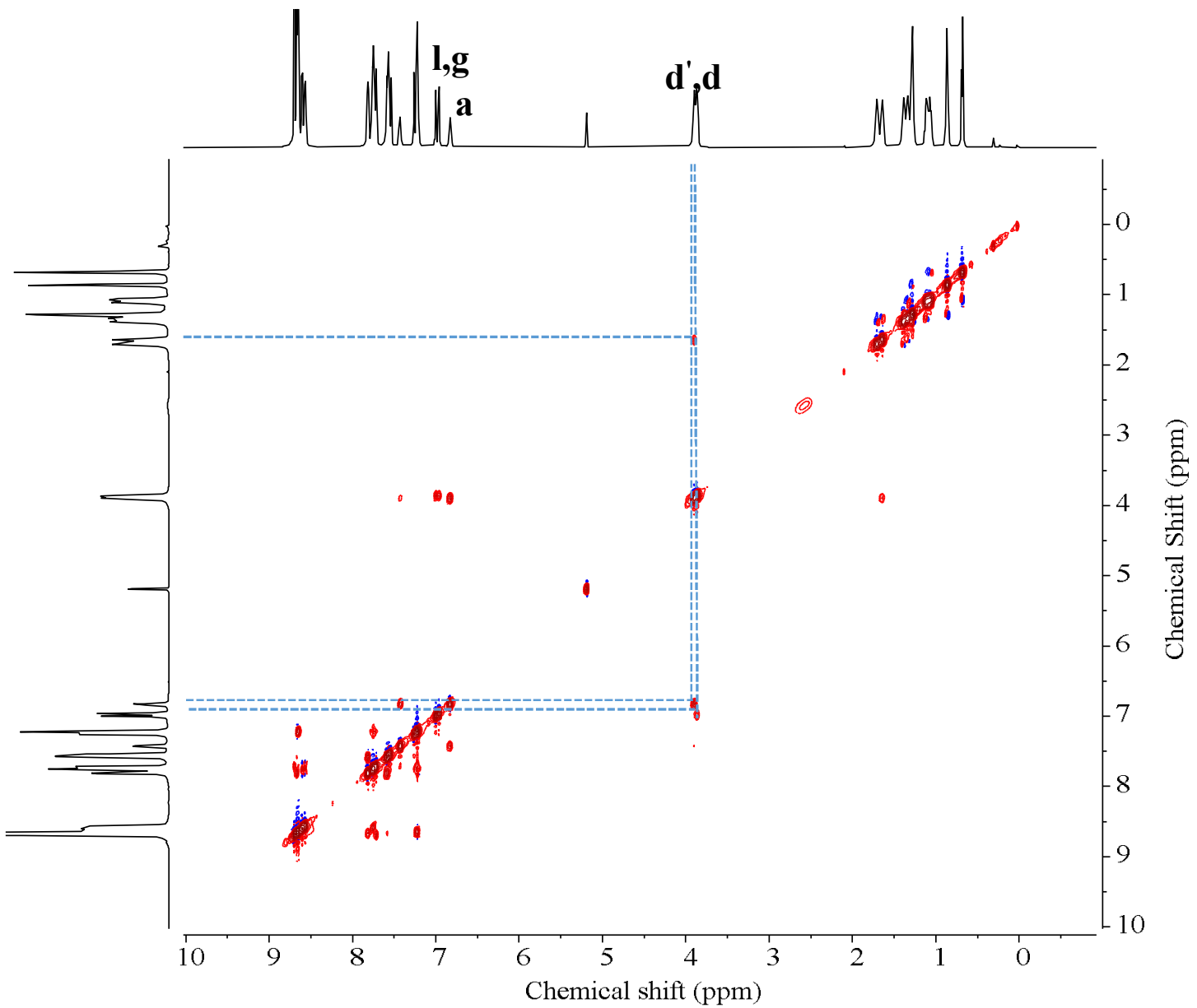

Figure S58. 2D NOESY NMR (600 MHz, $\left.\mathrm{CDCl}_{3}\right)$ spectrum of compound 13. 

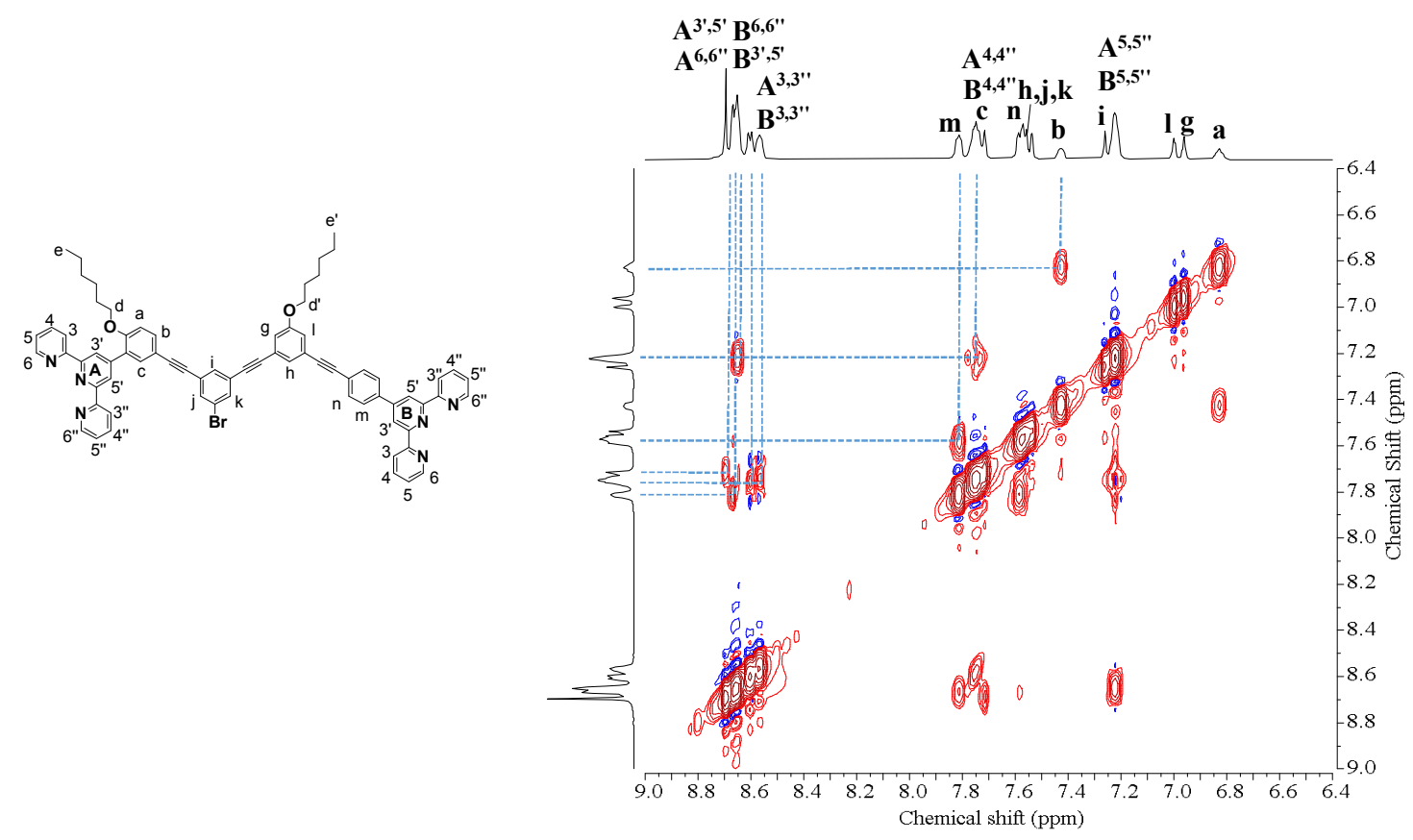

Figure S59. 2D NOESY NMR (600 $\left.\mathrm{MHz}, \mathrm{CDCl}_{3}\right)$ spectrum of compound $\mathbf{1 3}$ (aromatic region).

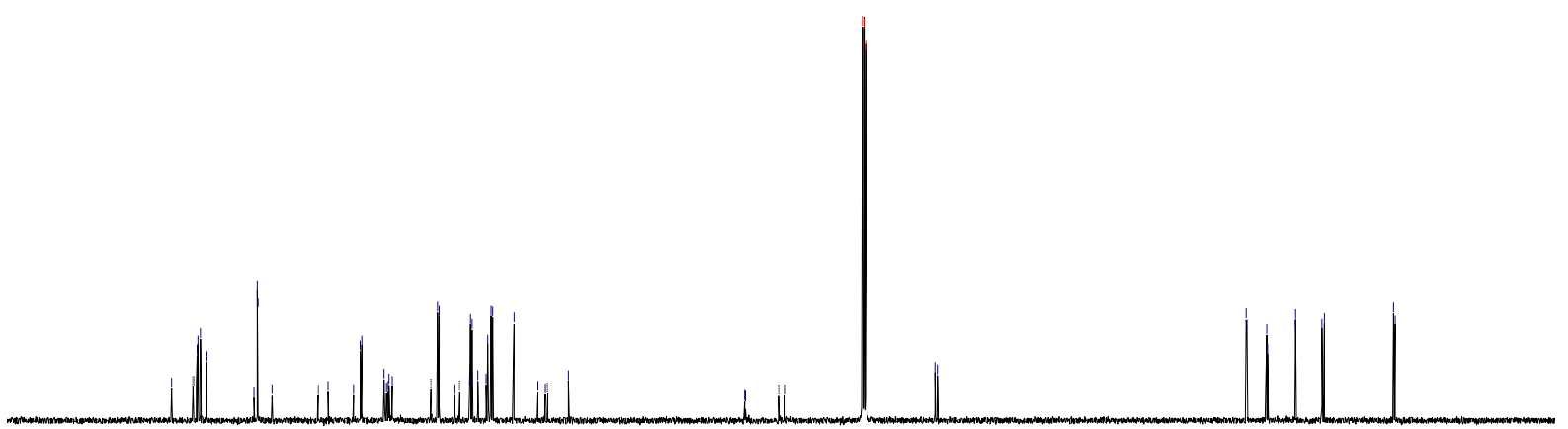

$\begin{array}{llllllllllllllllll}170 & 160 & 150 & 140 & 130 & 120 & 110 & \begin{array}{l}100 \\ \text { Chemical shift (ppm) }\end{array}\end{array}$

Figure S60. ${ }^{13} \mathrm{C} \mathrm{NMR}\left(150 \mathrm{MHz}, \mathrm{CDCl}_{3}\right)$ spectrum of compound 13. 


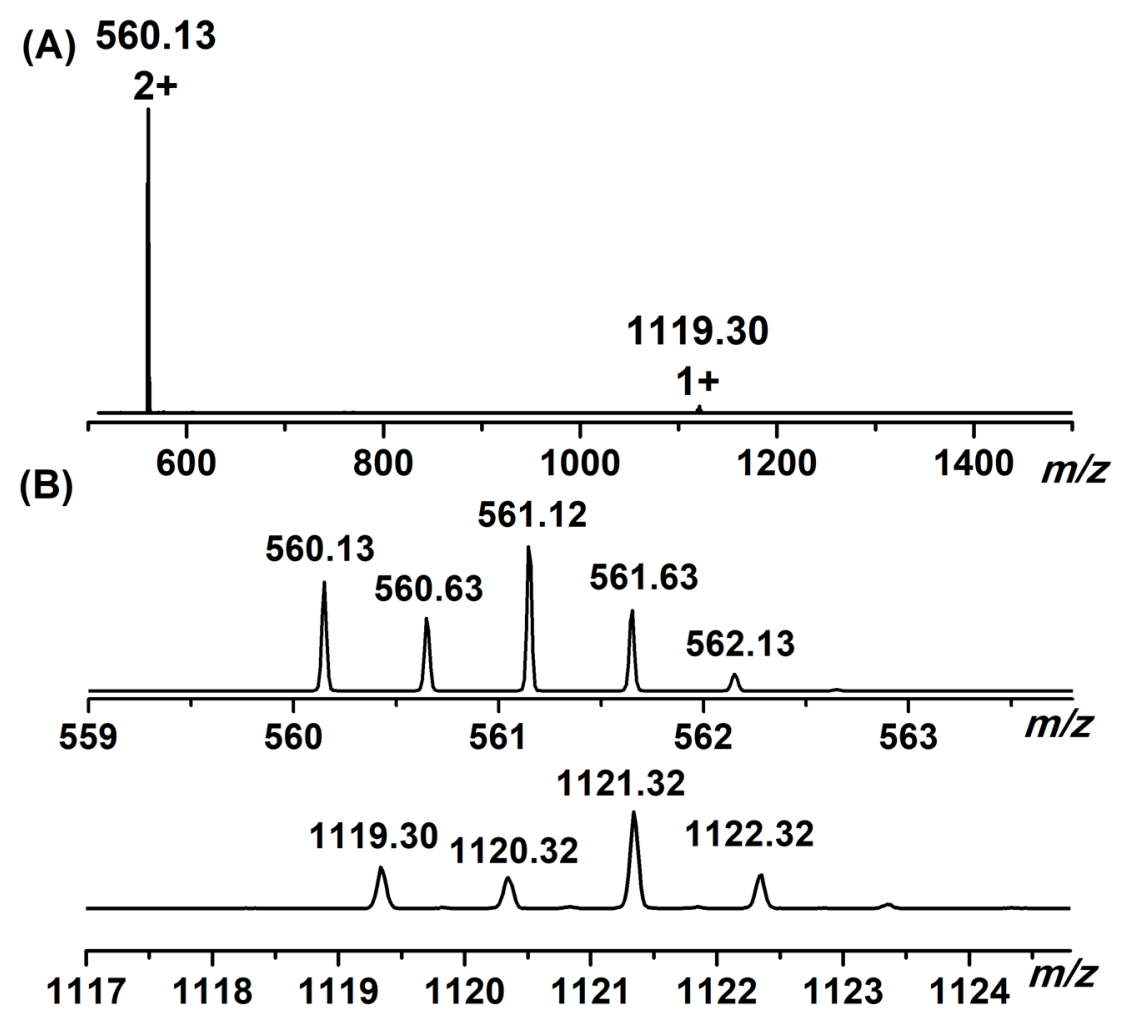

Figure S61. (A) ESI-MS spectrum of compound 13 in $\mathrm{CHCl}_{3} / \mathrm{CH}_{3} \mathrm{OH}(1 / 3)$ and (B) isotope patterns for different charge states.

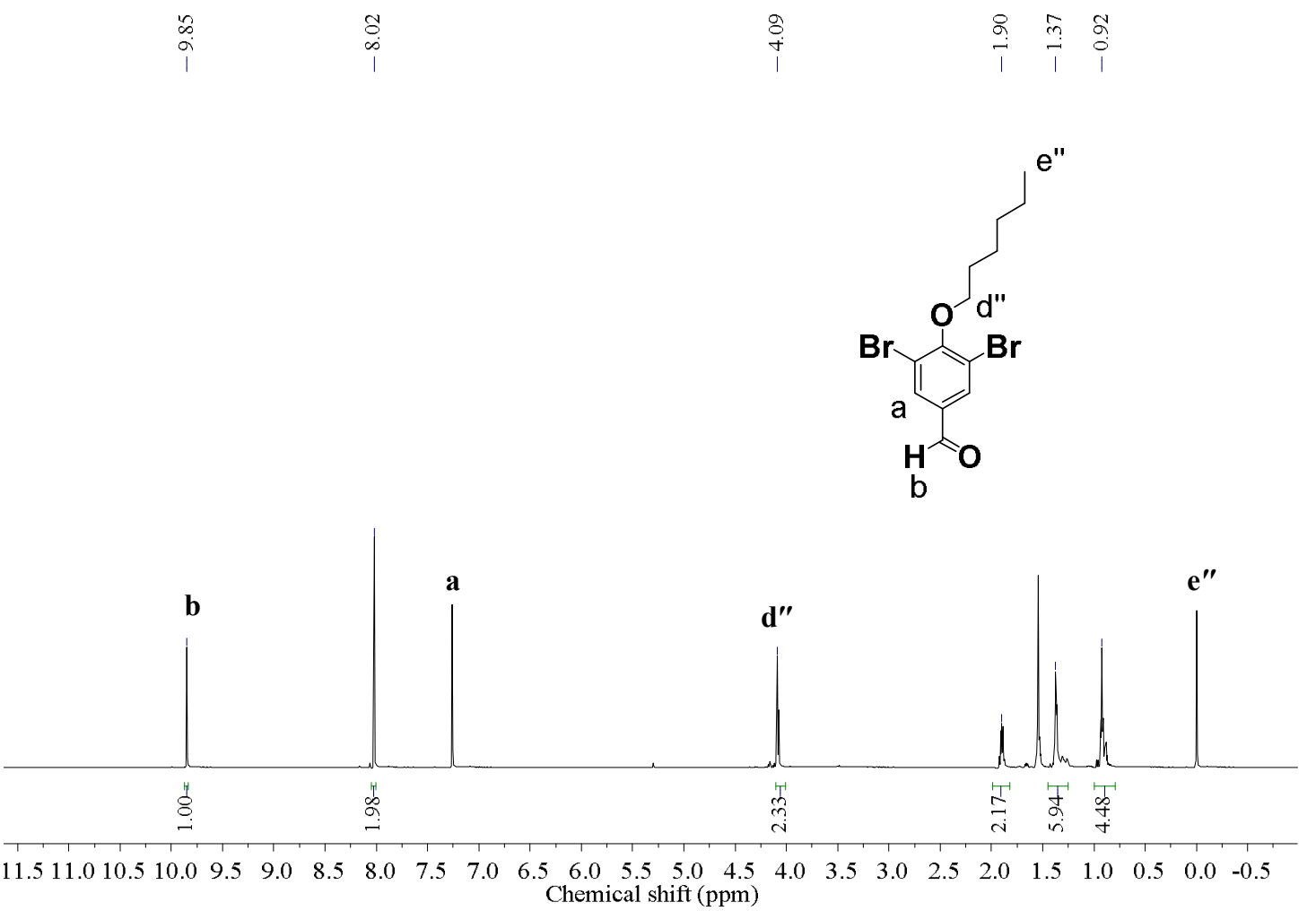

Figure S62. ${ }^{1} \mathrm{H}$ NMR $\left(600 \mathrm{MHz}, \mathrm{CDCl}_{3}\right)$ spectrum of compound 14. 


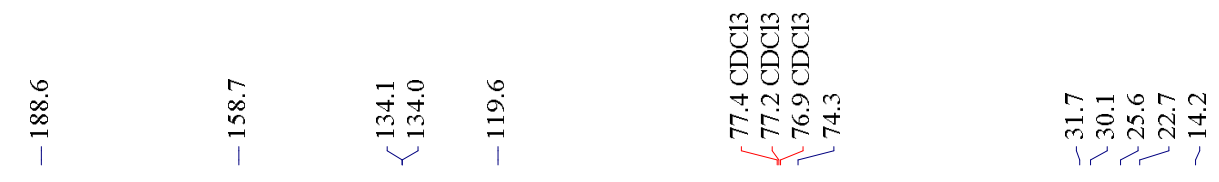

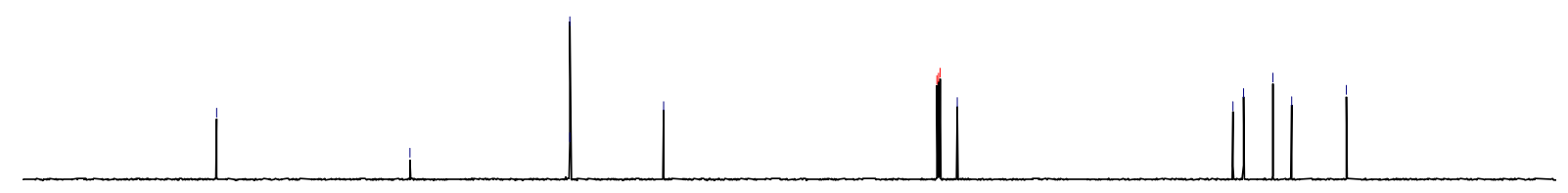

$\begin{array}{llllllllllllllllllllllllll}210 & 200 & 190 & 180 & 170 & 160 & 150 & 140 & 130 & 120 & 110 & 100 & 90 & 80 & 70 & 60 & 50 & 40 & 30 & 20 & 10 & 0 & -10 \\ \text { Chemical shift (ppm) }\end{array}$

Figure S63. ${ }^{13} \mathrm{C}$ NMR $\left(150 \mathrm{MHz}, \mathrm{CDCl}_{3}\right)$ spectrum of compound $\mathbf{1 4}$.

$\frac{m}{\mathrm{U}}$

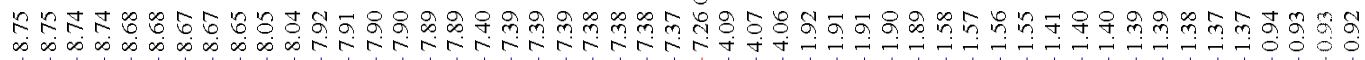

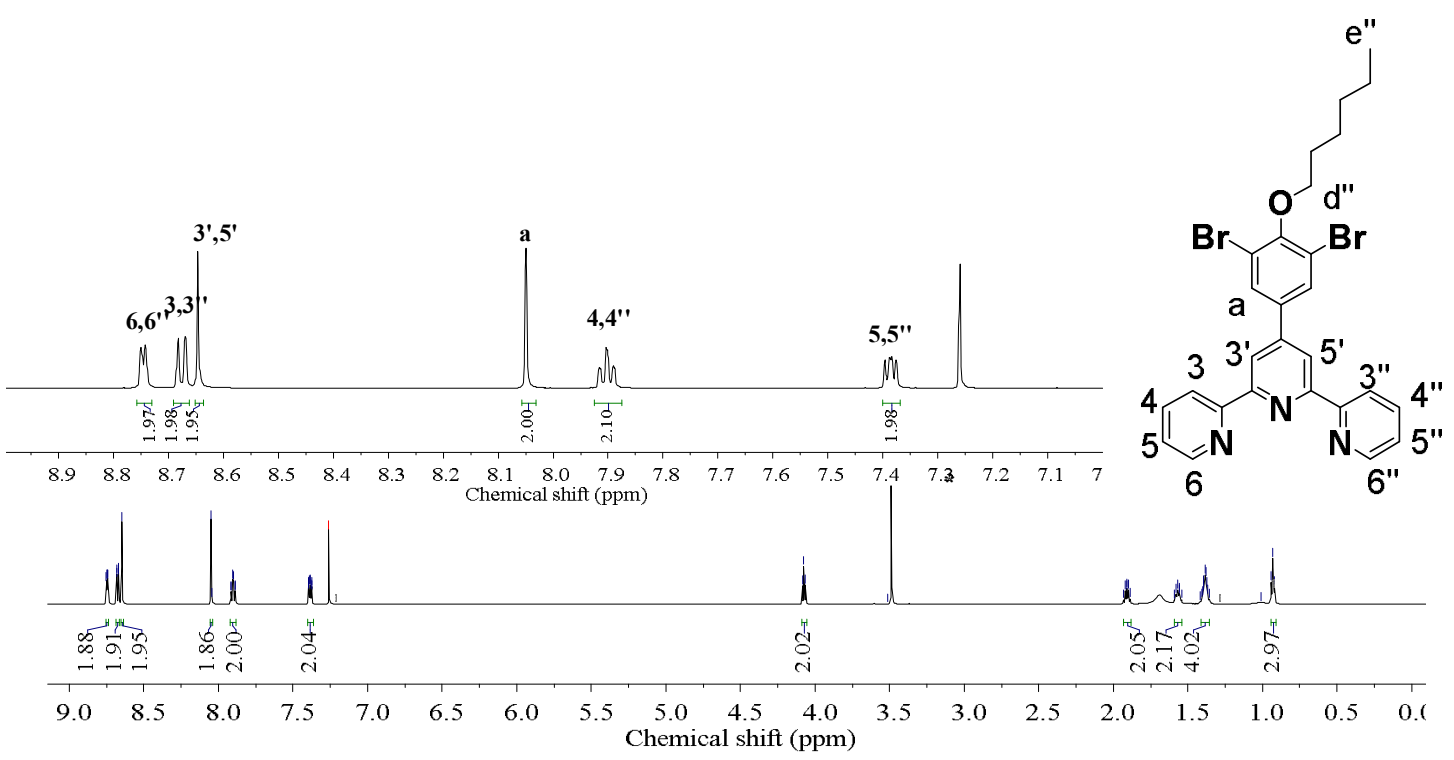

Figure S64. ${ }^{1} \mathrm{H}$ NMR $\left(600 \mathrm{MHz}, \mathrm{CDCl}_{3}\right)$ spectrum of compound 15. 


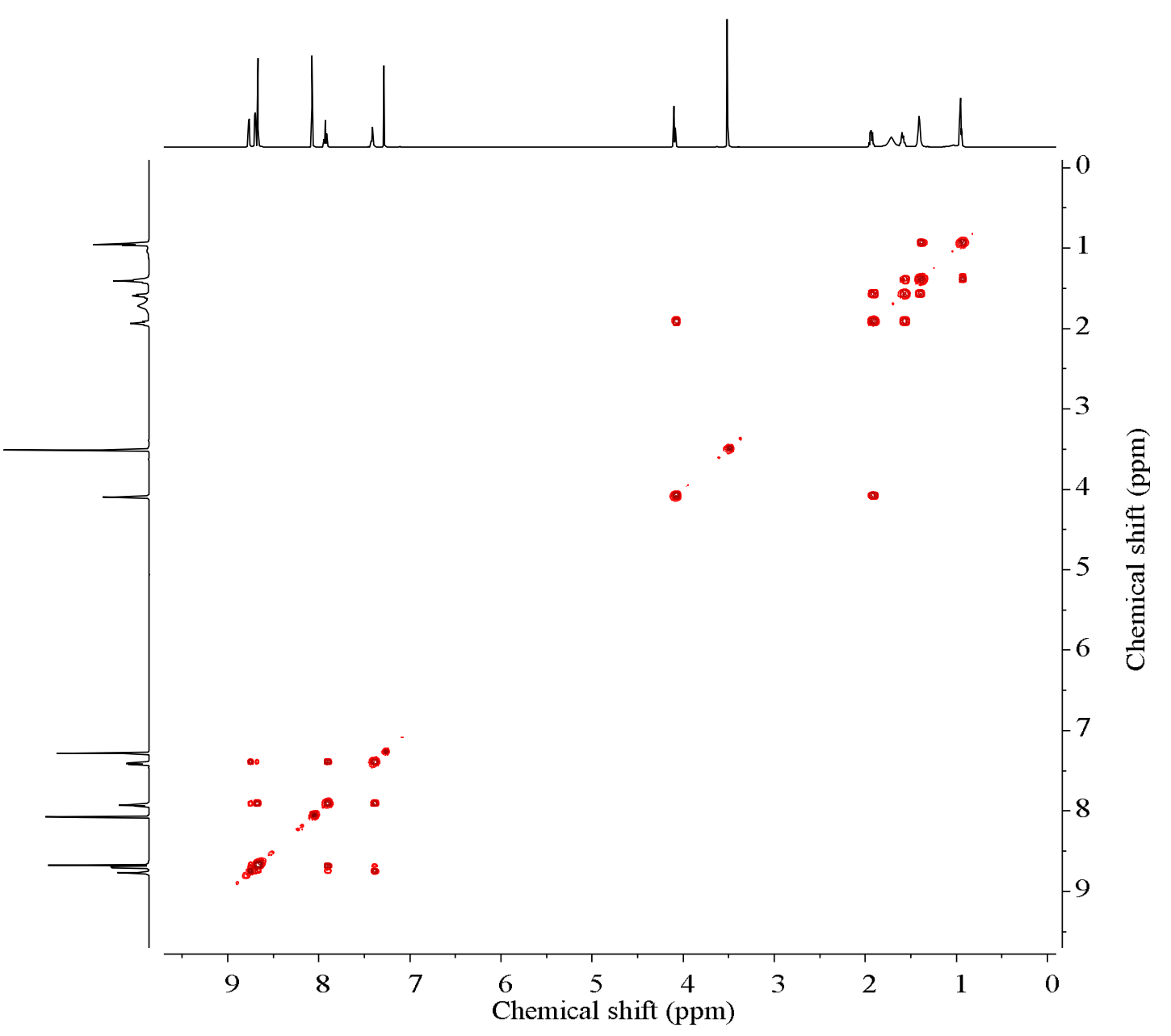

Figure S65. 2D COSY NMR (600 MHz, $\left.\mathrm{CDCl}_{3}\right)$ spectrum of compound 15.
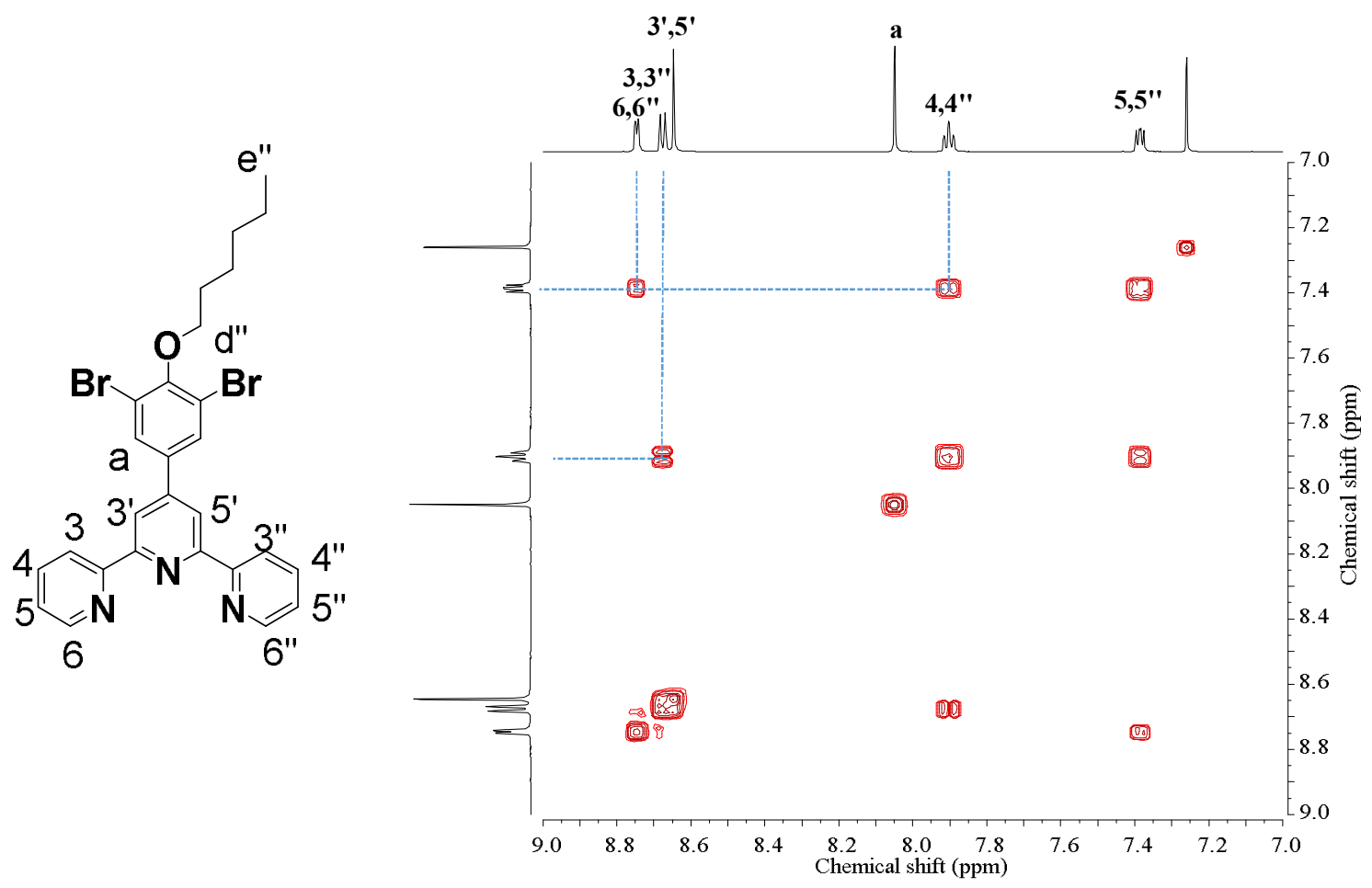

Figure S66. 2D COSY NMR (600 MHz, $\mathrm{CDCl}_{3}$ ) spectrum of compound $\mathbf{1 5}$ (aromatic 
region).

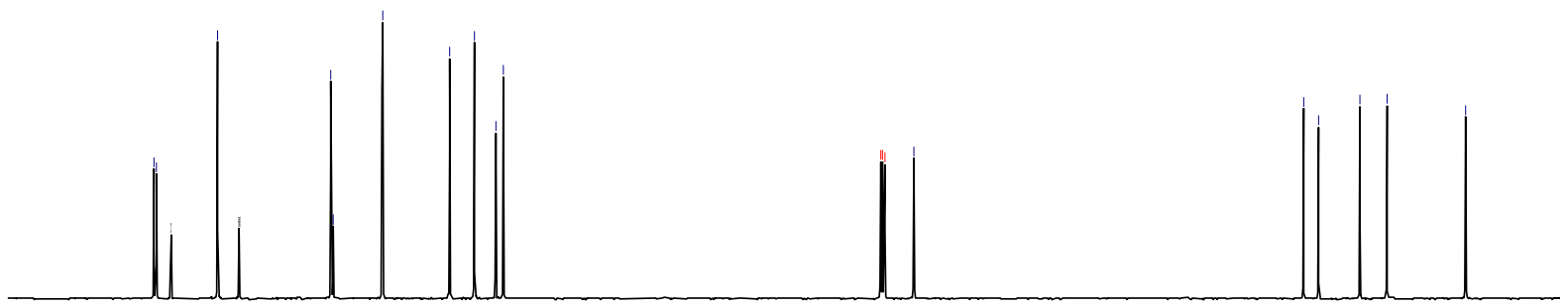

\begin{tabular}{rllllllllllllll}
\hline 170 & 160 & 150 & 140 & 130 & 120 & 110 & $\begin{array}{l}100 \\
\text { Chemical shift }(\mathrm{ppm})\end{array}$
\end{tabular}

Figure S67. ${ }^{13} \mathrm{C} \mathrm{NMR}\left(150 \mathrm{MHz}, \mathrm{CDCl}_{3}\right)$ spectrum of compound 15.

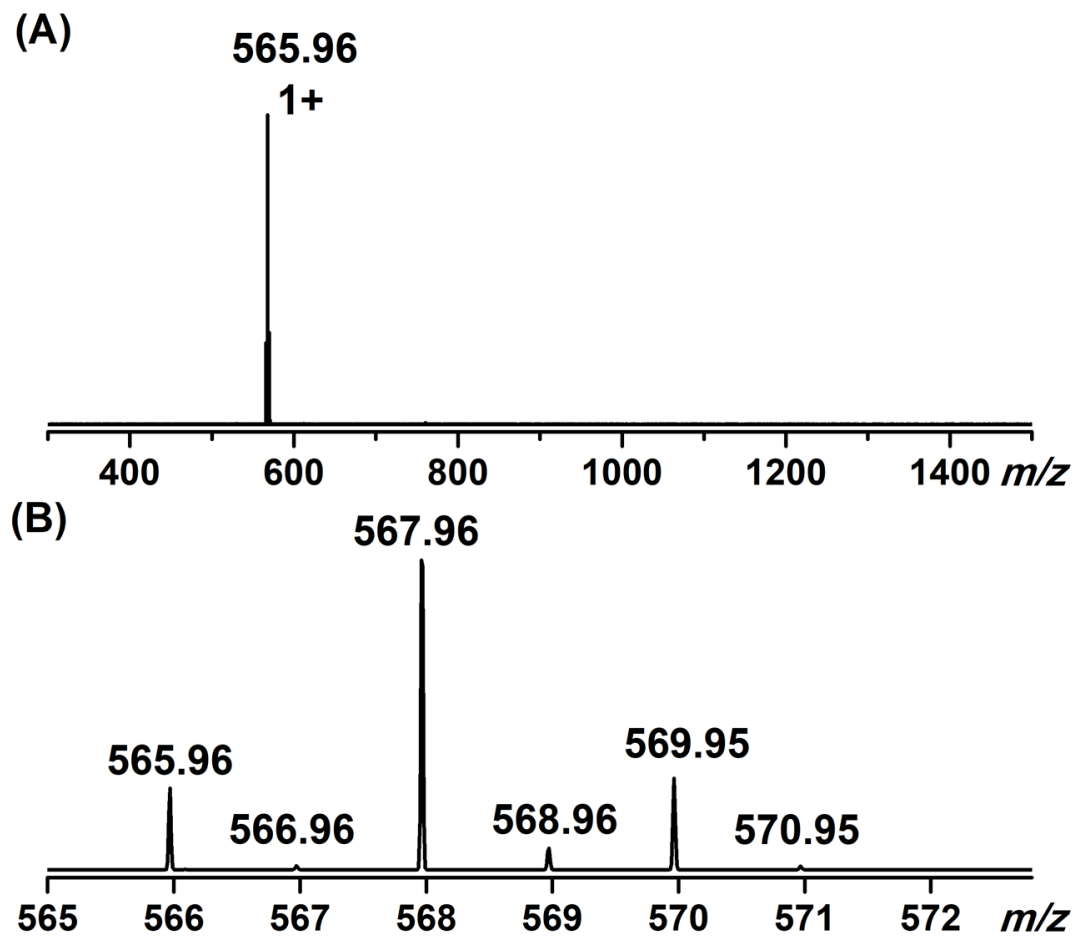

Figure S68. (A) ESI-MS spectrum of compound 15 in $\mathrm{CHCl}_{3} / \mathrm{CH}_{3} \mathrm{OH}(1 / 3)$ and (B) isotope pattern. 


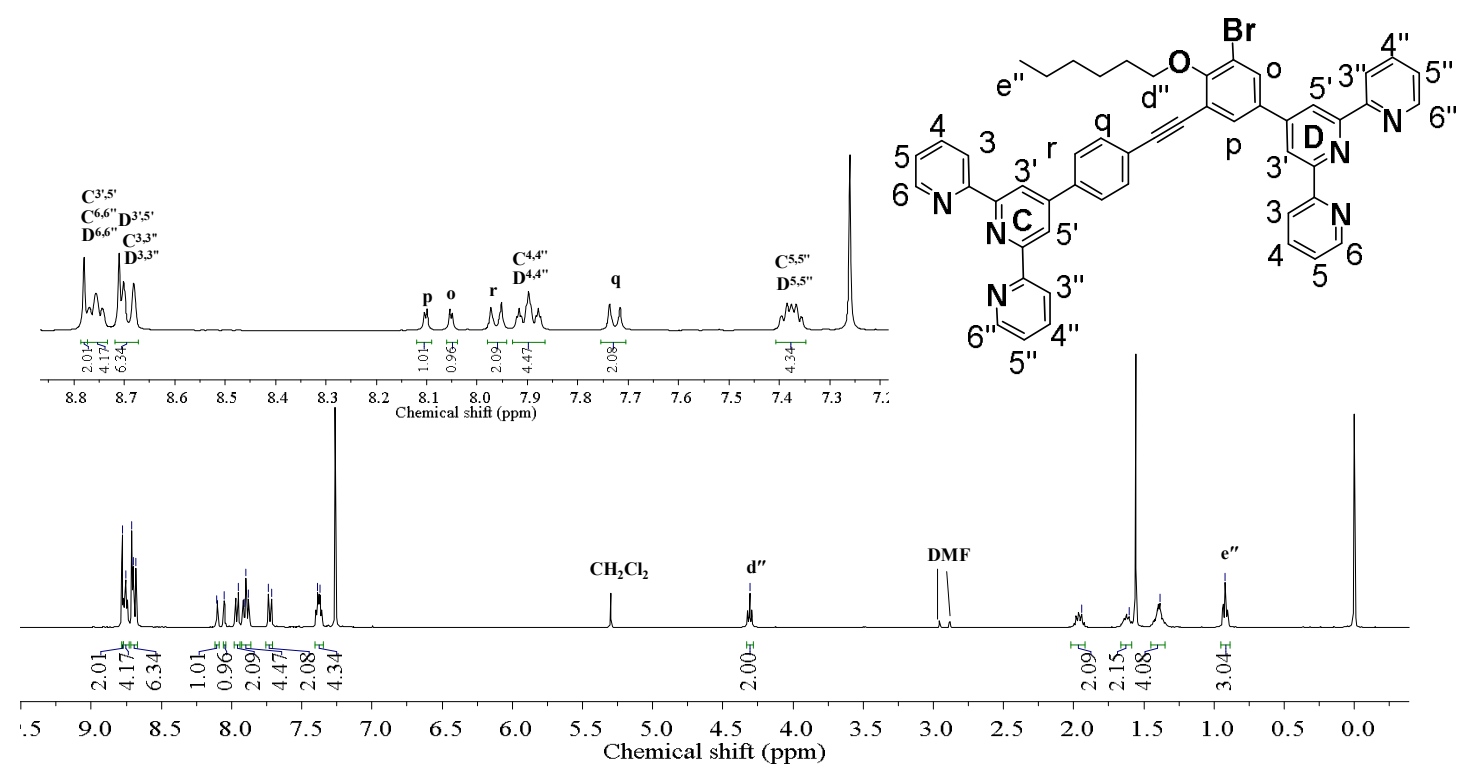

Figure S69. ${ }^{1} \mathrm{H}$ NMR (400 MHz, $\left.\mathrm{CDCl}_{3}\right)$ spectrum of compound 16.

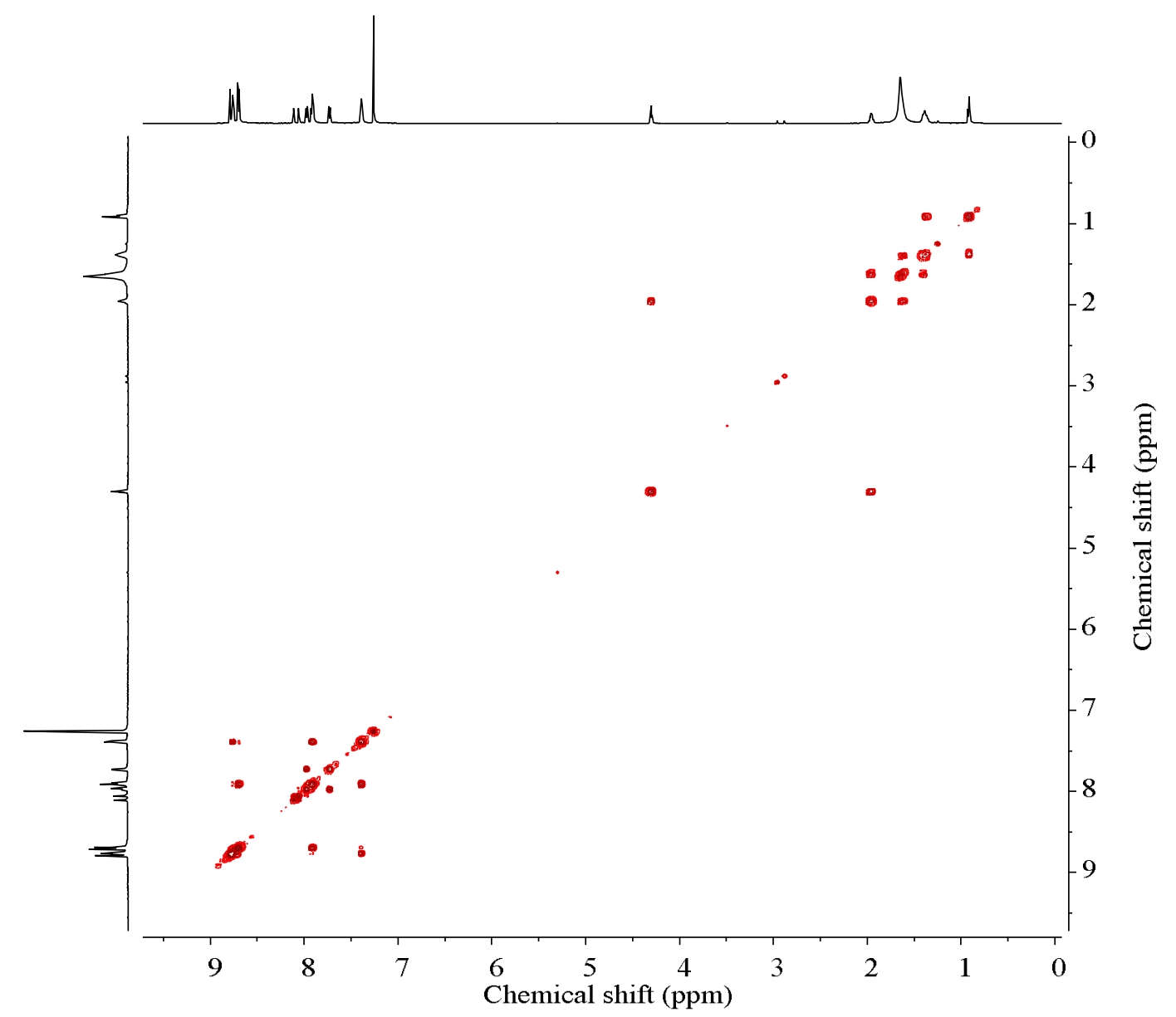

Figure S70. 2D COSY NMR (600 MHz, $\left.\mathrm{CDCl}_{3}\right)$ spectrum of compound 16. 


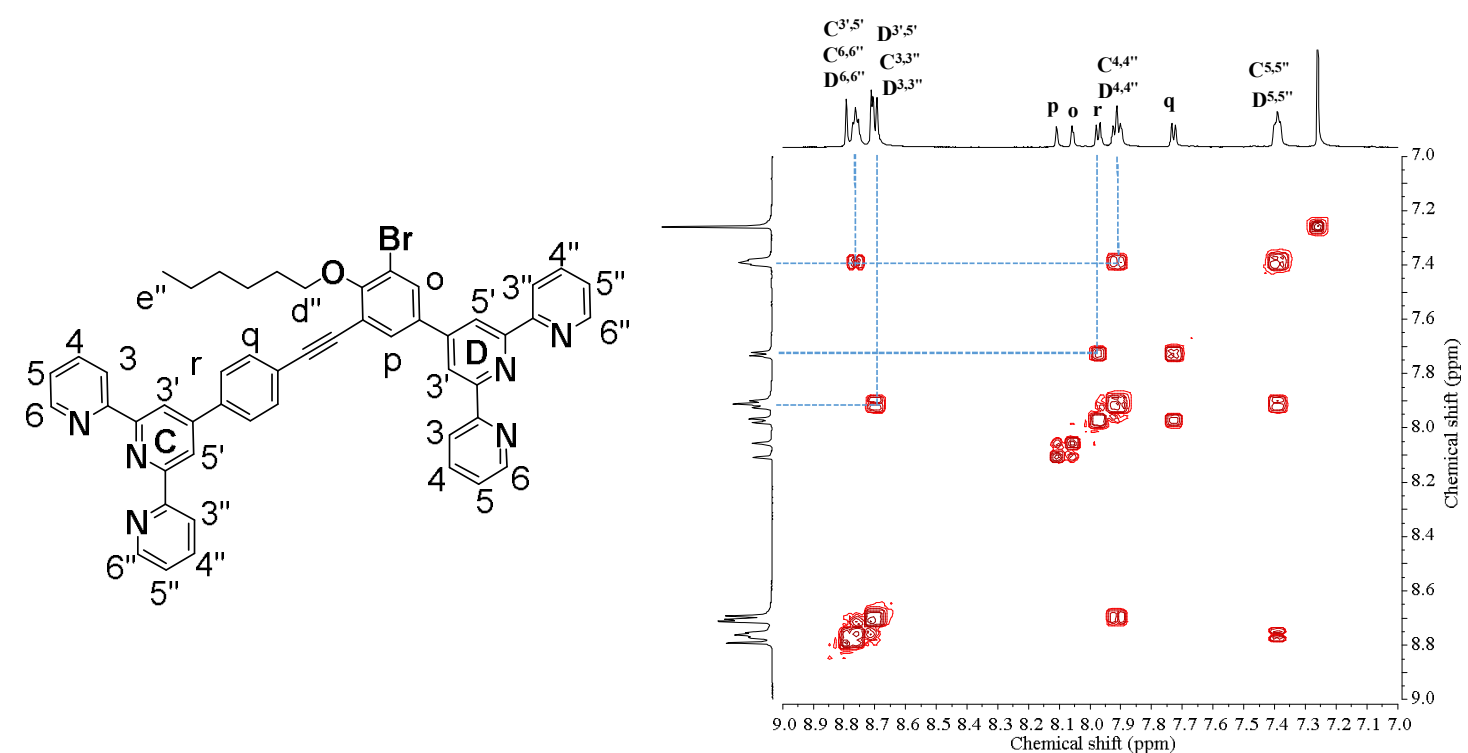

Figure S71. 2D COSY NMR (600 $\left.\mathrm{MHz}, \mathrm{CDCl}_{3}\right)$ spectrum of compound $\mathbf{1 6}$ (aromatic region).

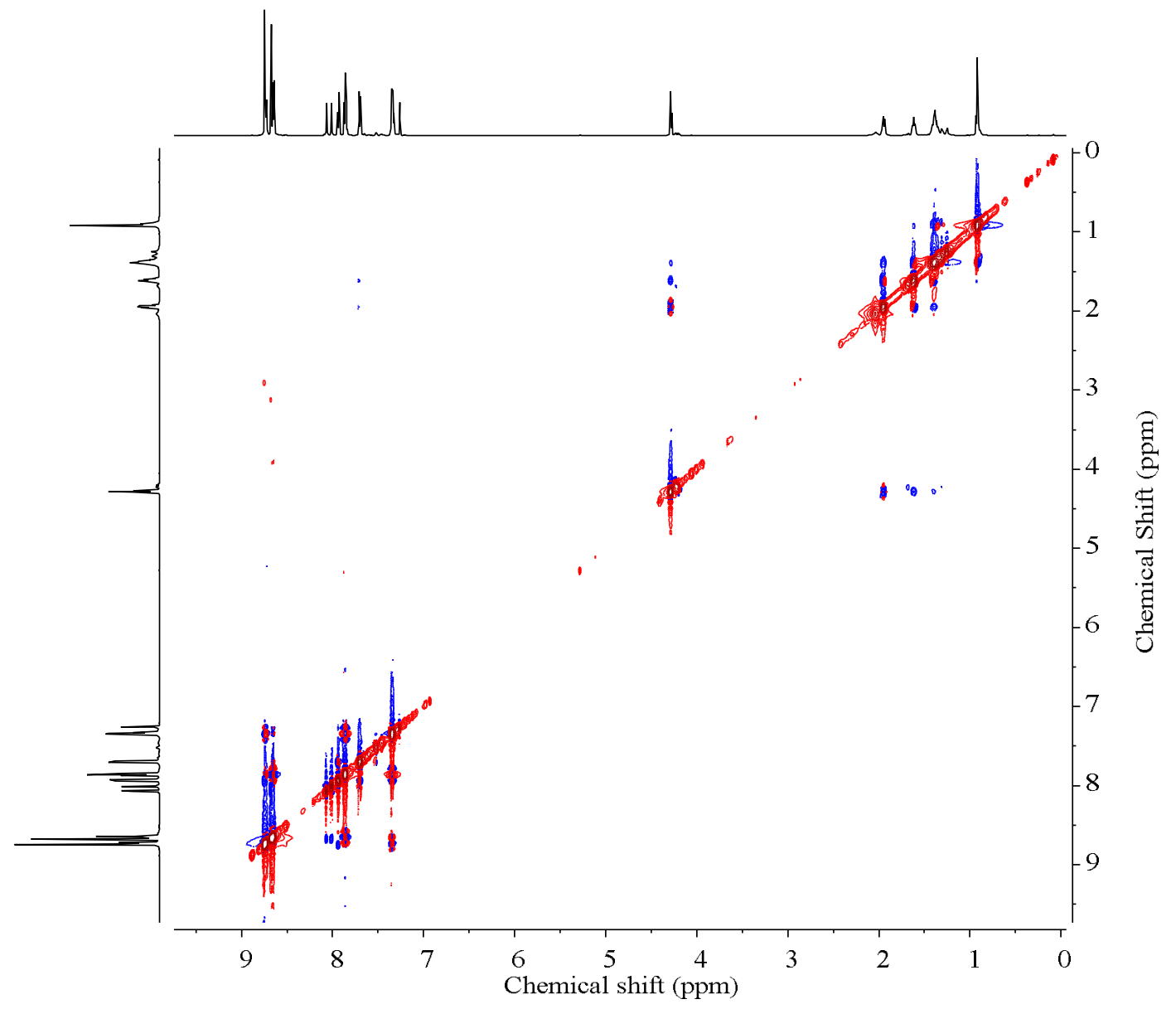

Figure S72. 2D NOESY NMR (600 MHz, $\mathrm{CDCl}_{3}$ ) spectrum of compound 16. 


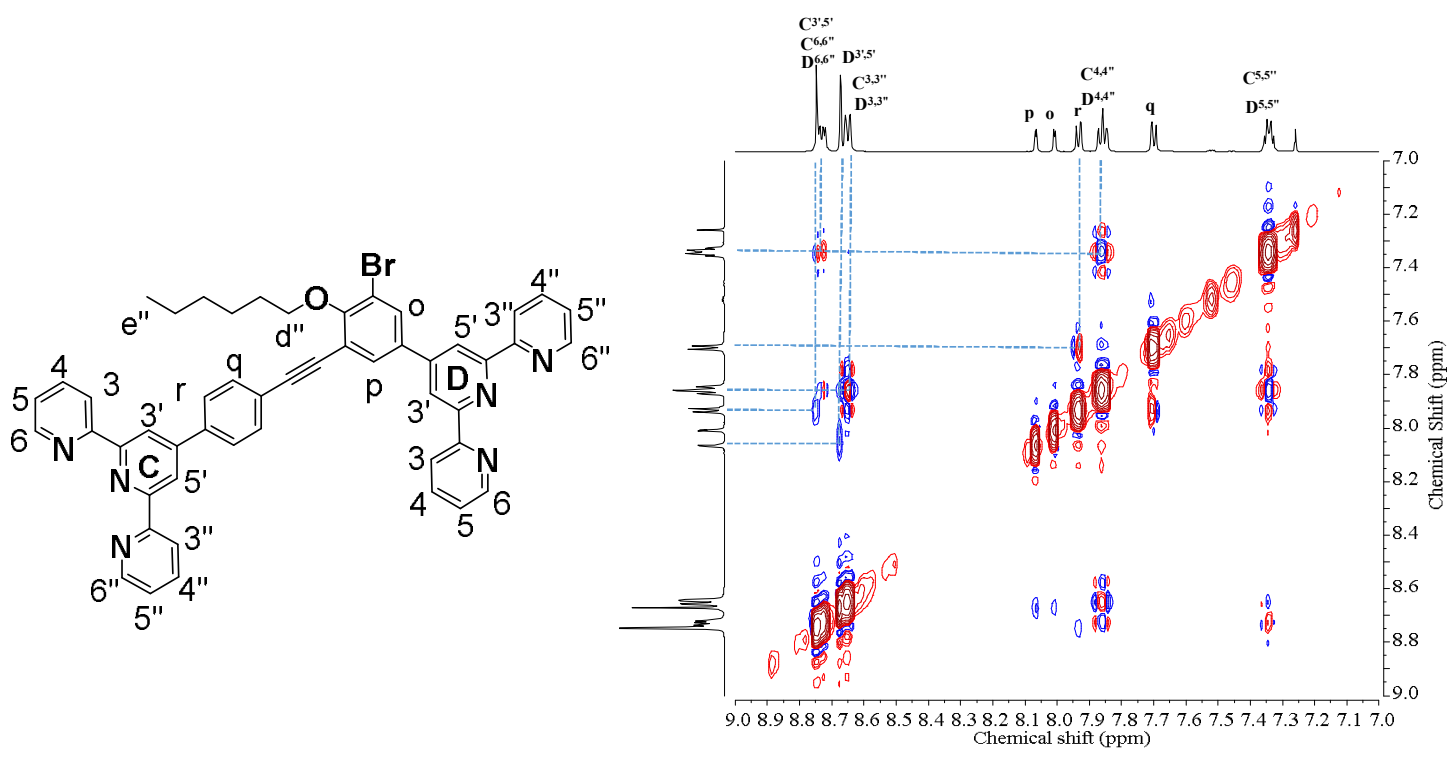

Figure S73. 2D NOESY NMR (600 MHz, $\left.\mathrm{CDCl}_{3}\right)$ spectrum of compound $\mathbf{1 6}$ (aromatic region).
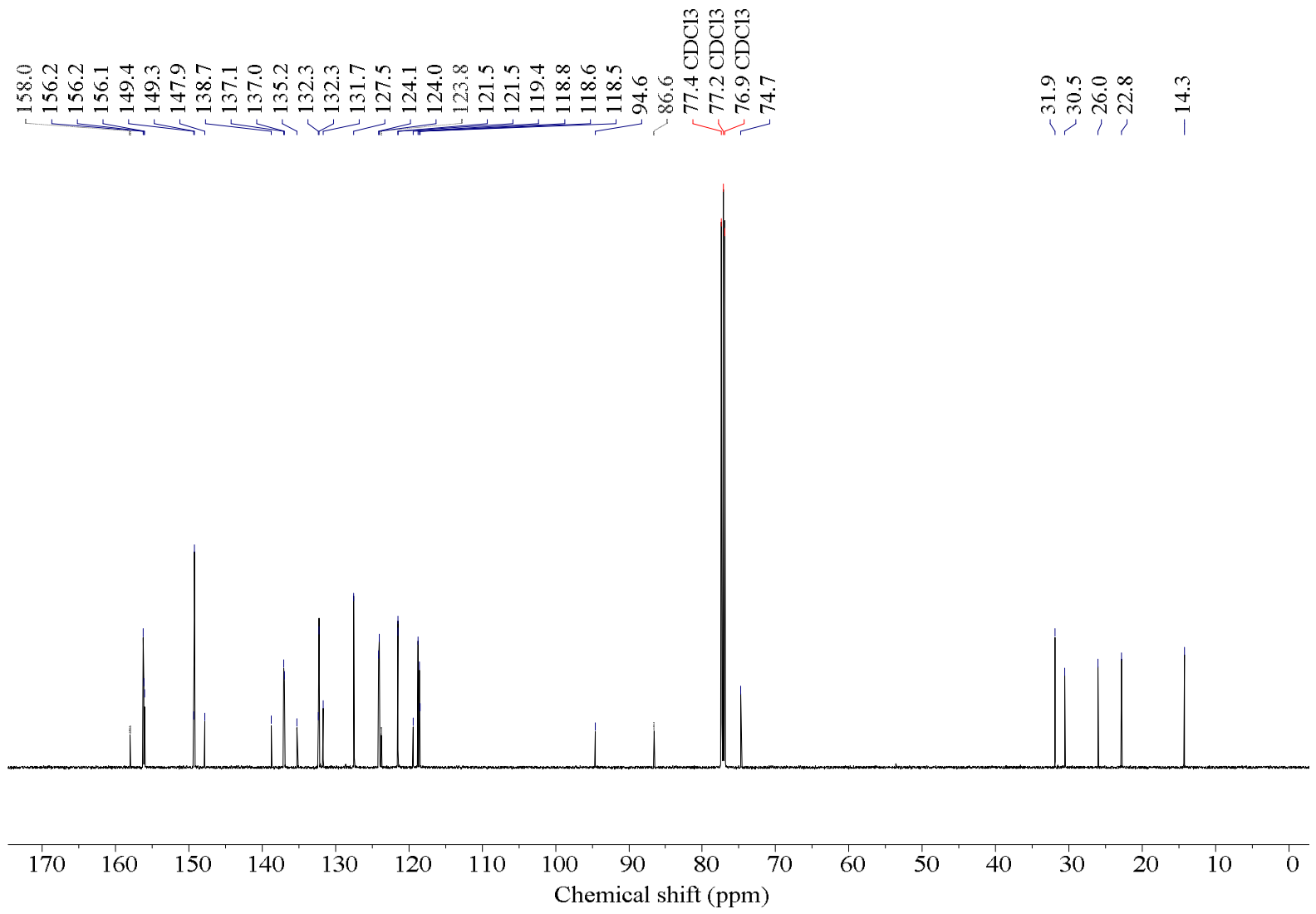

Figure S74. ${ }^{13} \mathrm{C}$ NMR $\left(150 \mathrm{MHz}, \mathrm{CDCl}_{3}\right)$ spectrum of compound $\mathbf{1 6}$. 
(A) 410.05

(B)
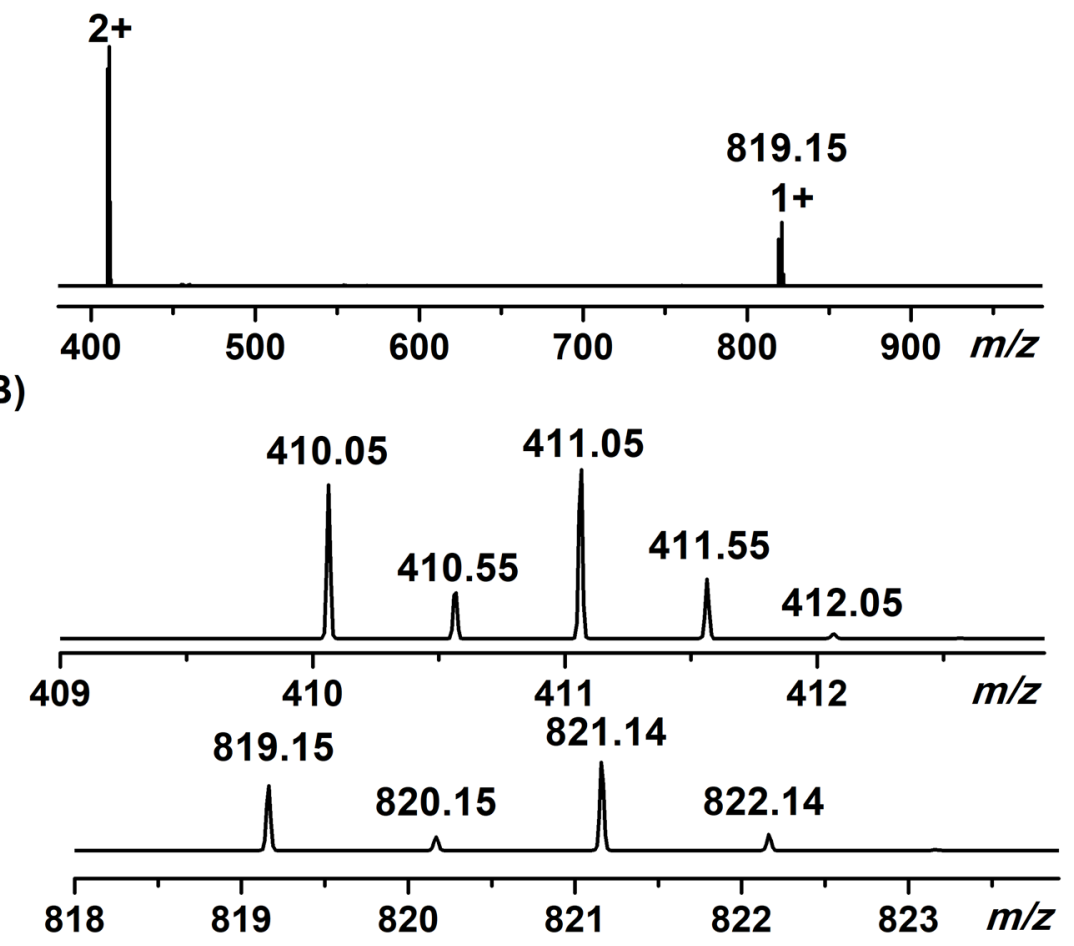

Figure S75. (A) ESI-MS spectrum of compound 16 in $\mathrm{CHCl}_{3} / \mathrm{CH}_{3} \mathrm{OH}(1 / 3)$ and (B) isotope patterns for different charge states.

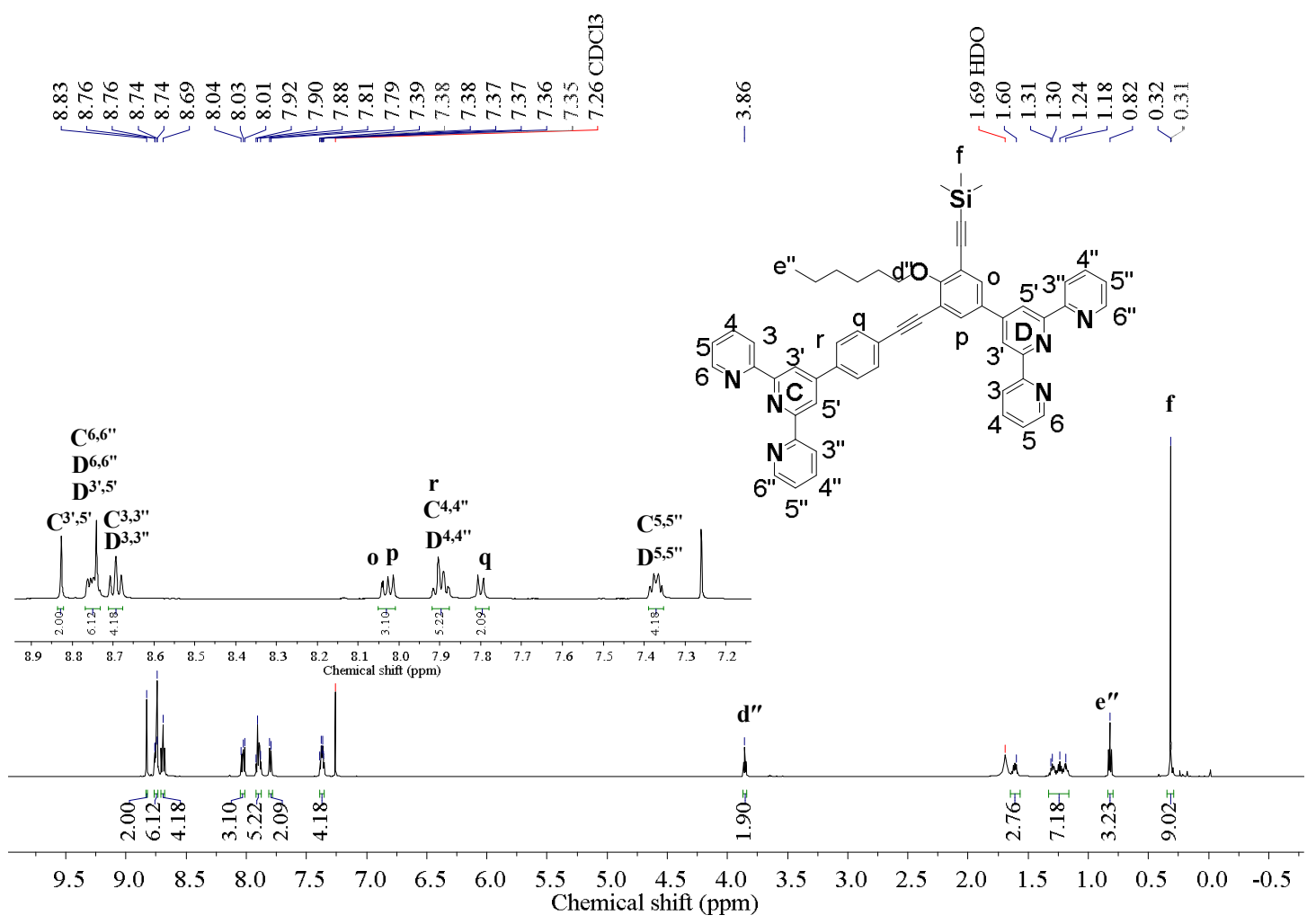

Figure S76. ${ }^{1} \mathrm{H}$ NMR $\left(600 \mathrm{MHz}, \mathrm{CDCl}_{3}\right)$ spectrum of compound 17. 


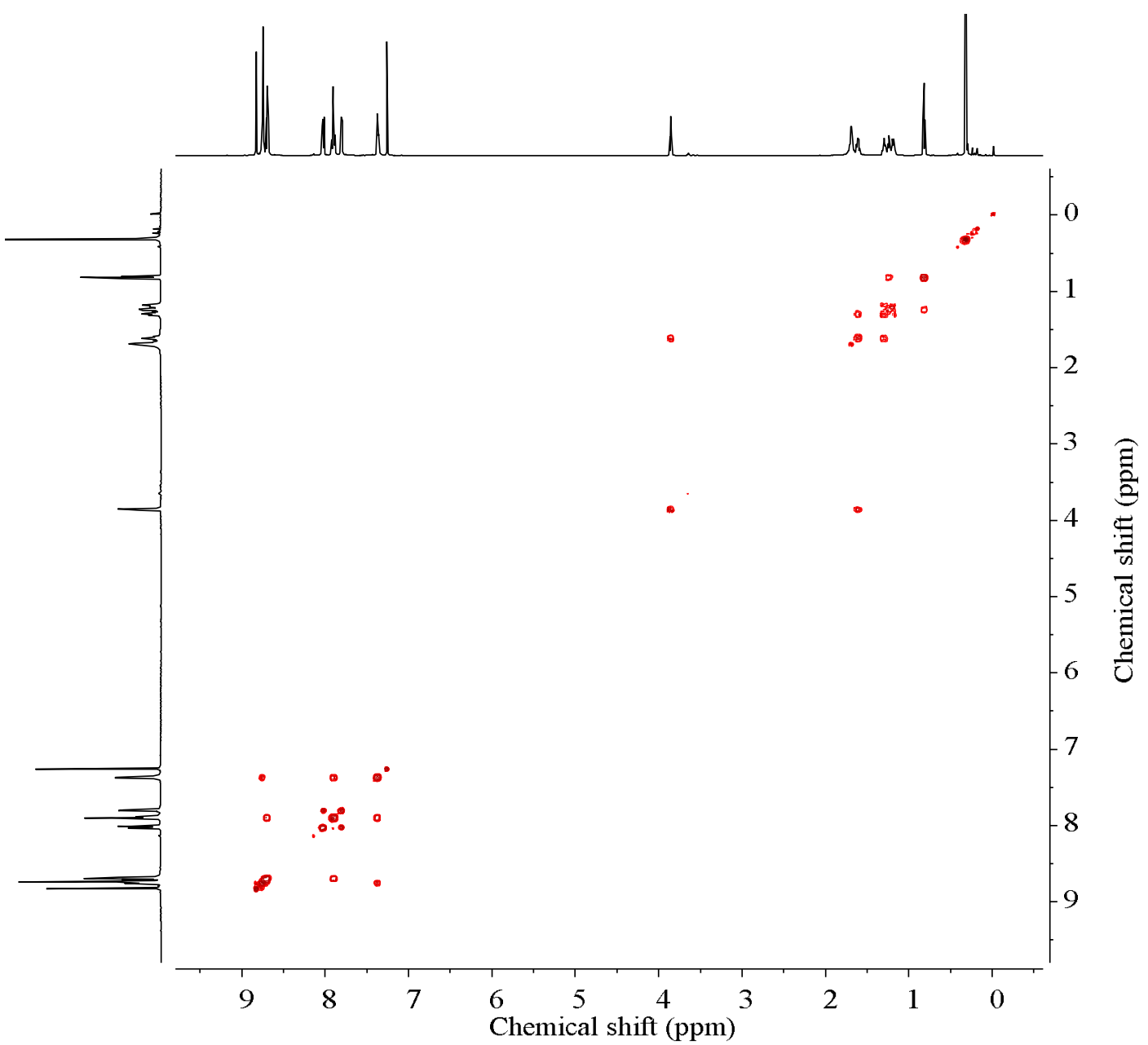

Figure S77. 2D COSY NMR (600 MHz, $\left.\mathrm{CDCl}_{3}\right)$ spectrum of compound 17.

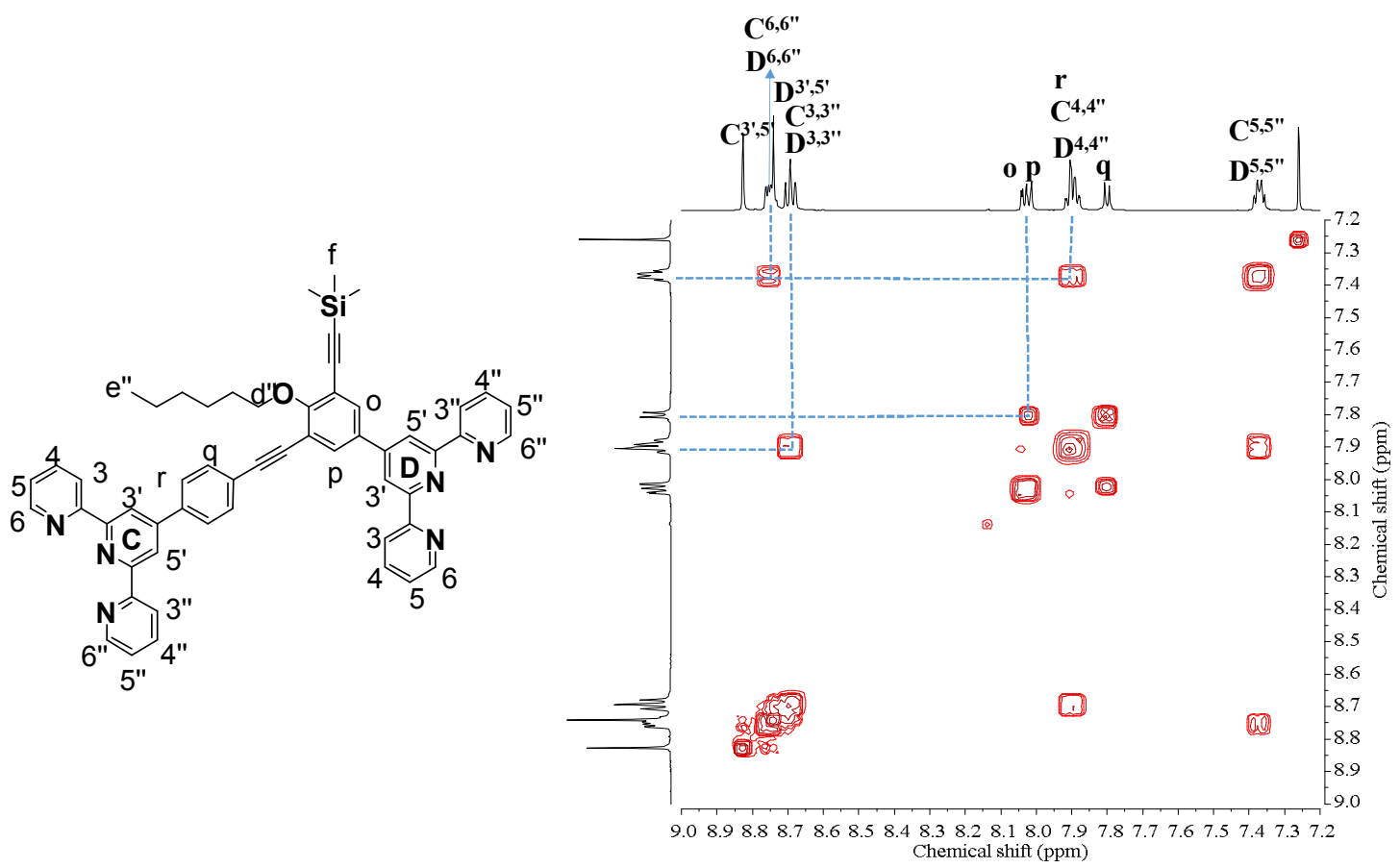

Figure S78. 2D COSY NMR (600 $\left.\mathrm{MHz}, \mathrm{CDCl}_{3}\right)$ spectrum of compound 17 (aromatic region). 


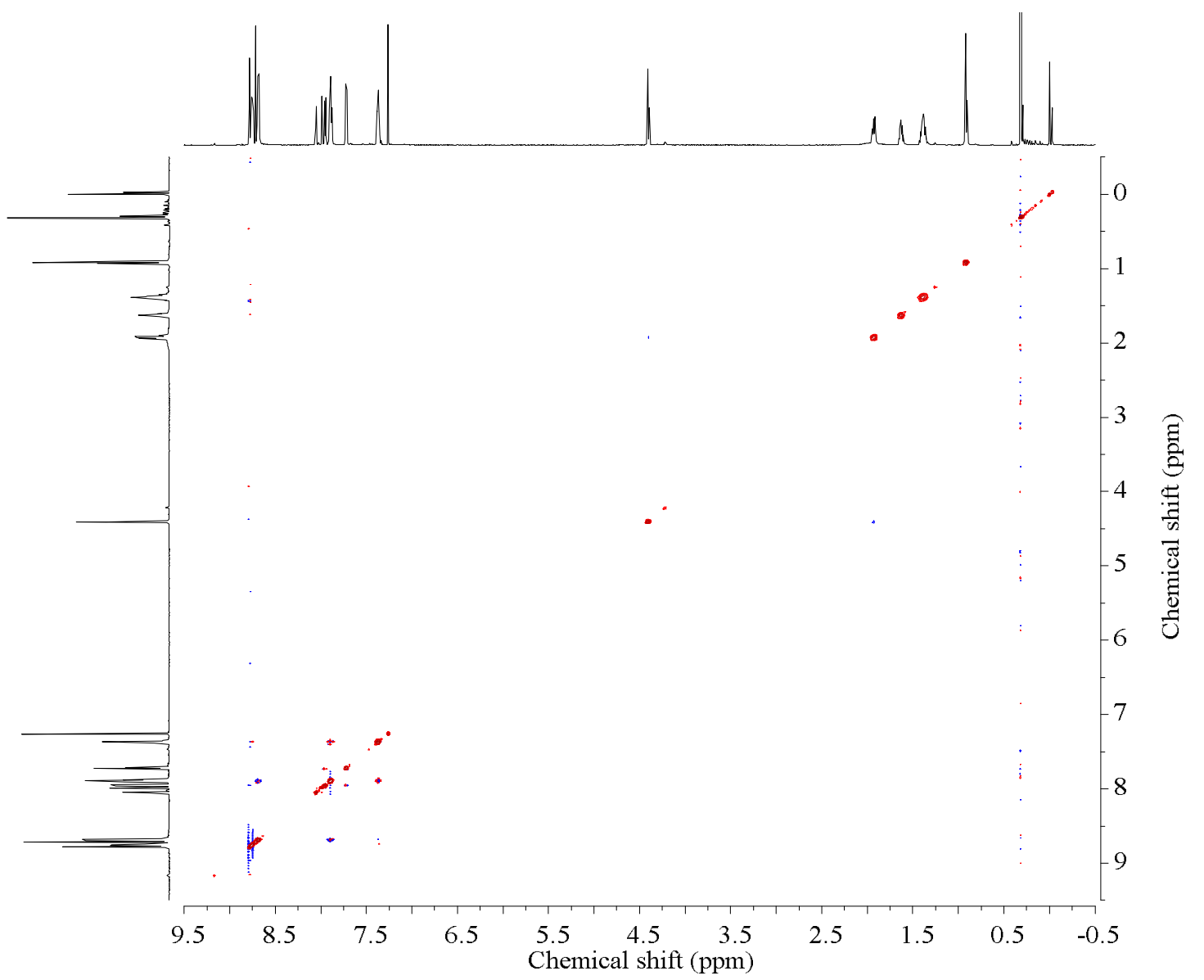

Figure S79. 2D NOESY NMR (600 MHz, $\left.\mathrm{CDCl}_{3}\right)$ spectrum of compound 17.

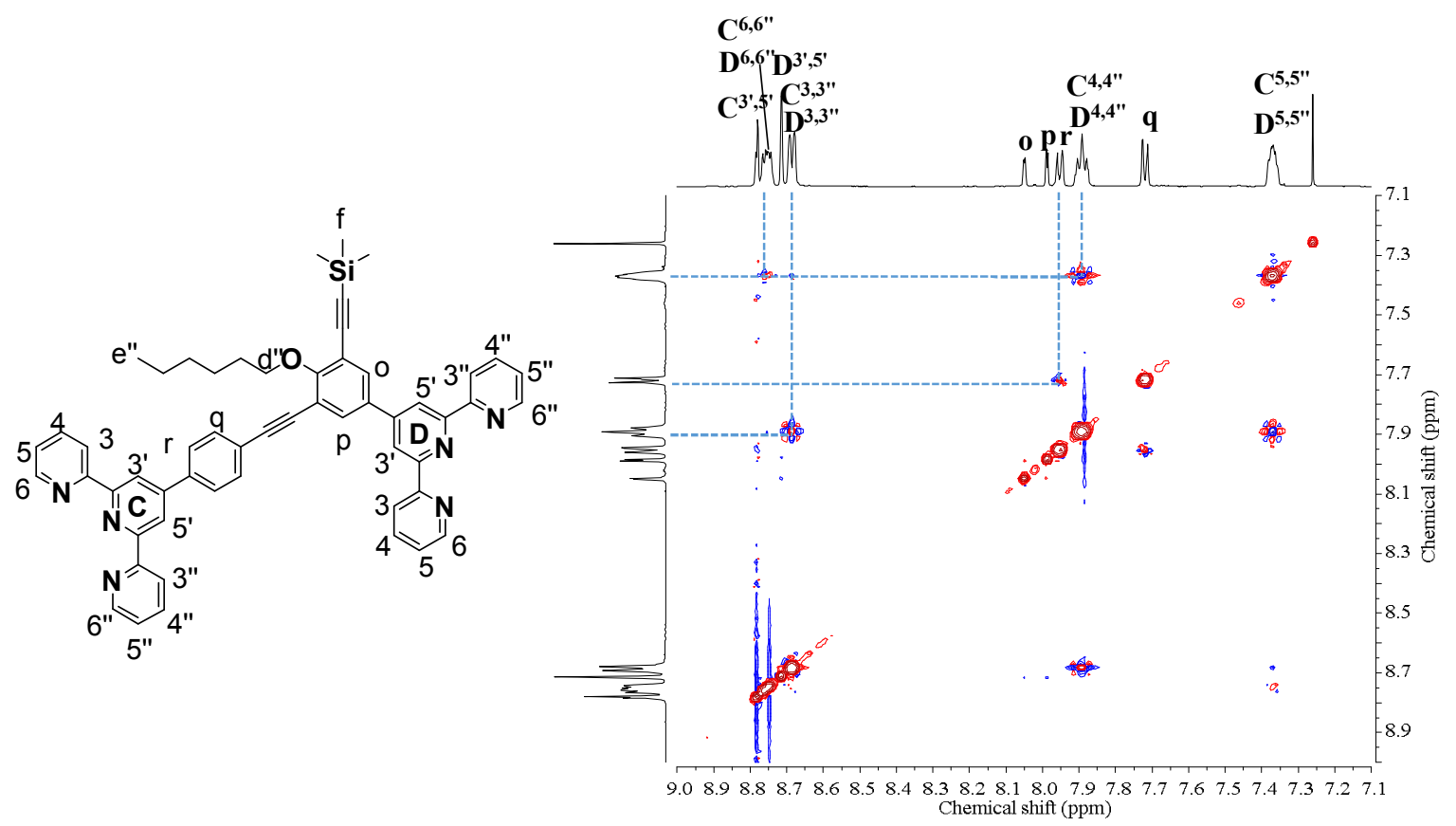

Figure S80. 2D NOESY NMR (600 MHz, $\left.\mathrm{CDCl}_{3}\right)$ spectrum of compound 17 (aromatic region). 

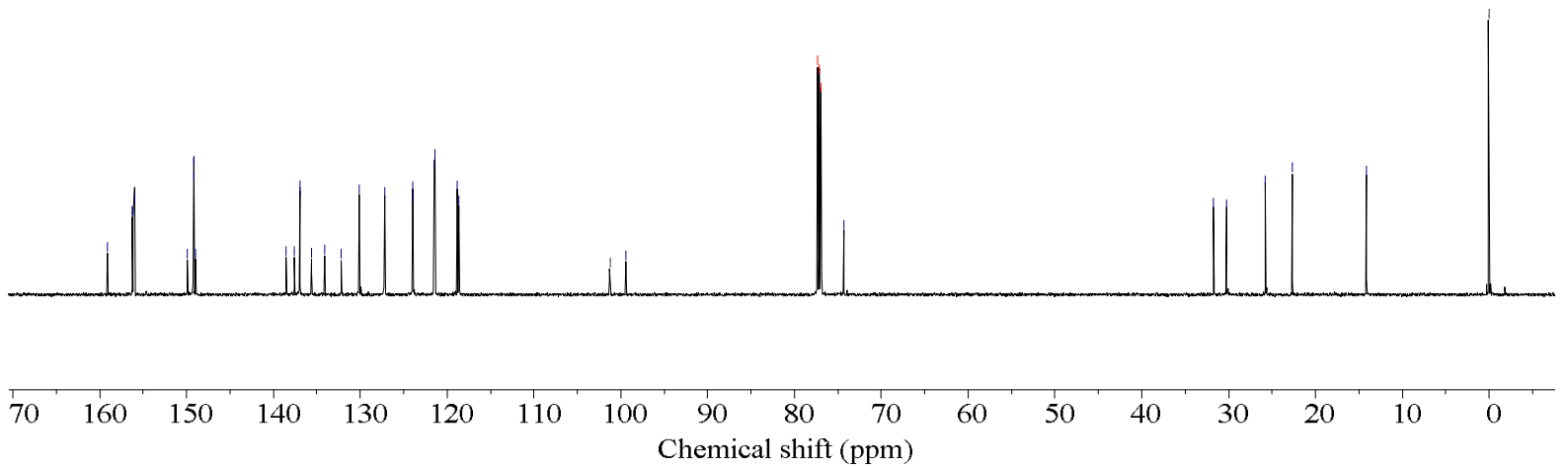

Figure S81. ${ }^{13} \mathrm{C}$ NMR $\left(150 \mathrm{MHz}, \mathrm{CDCl}_{3}\right)$ spectrum of compound 17.

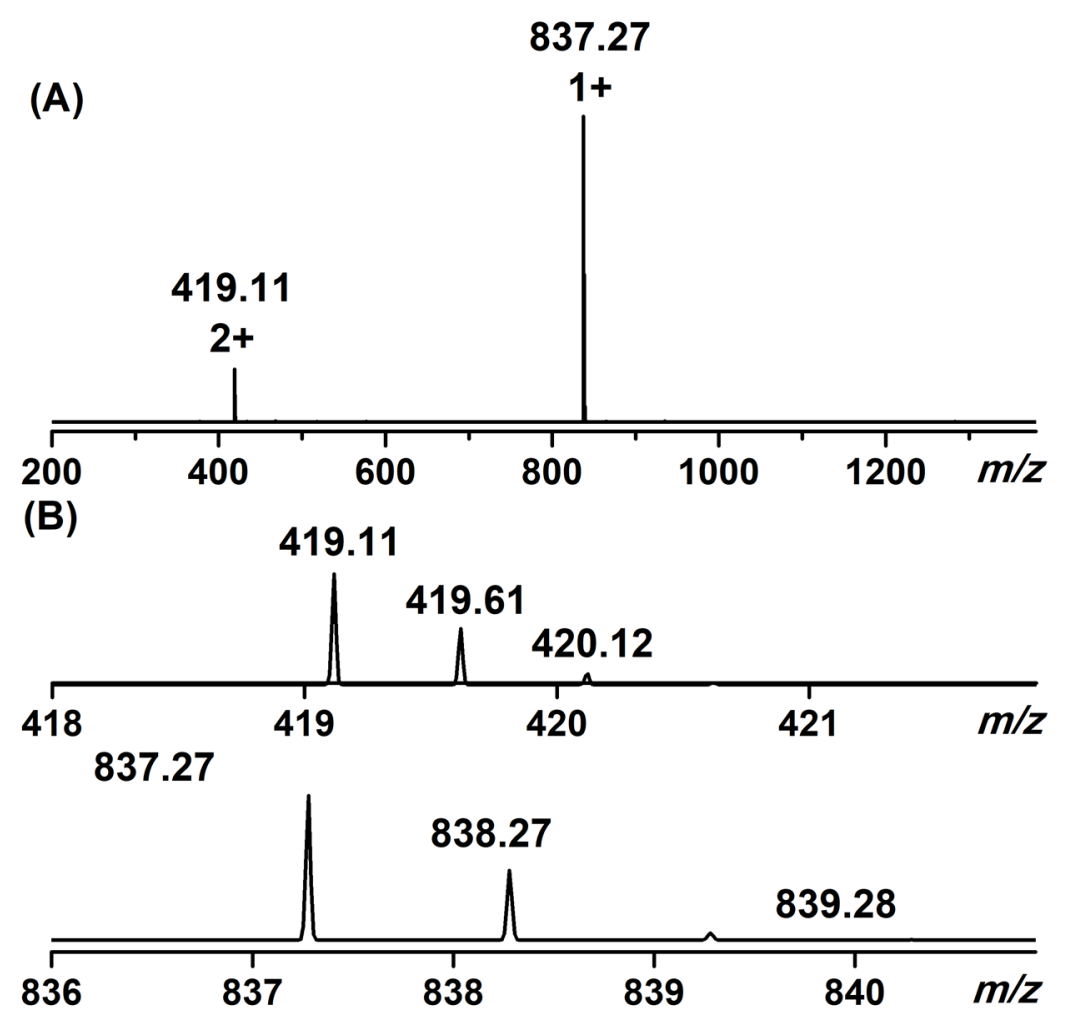

Figure S82. (A) ESI-MS spectrum of compound 17 in $\mathrm{CHCl}_{3} / \mathrm{CH}_{3} \mathrm{OH}(1 / 3)$ and (B) isotope patterns for different charge states. 


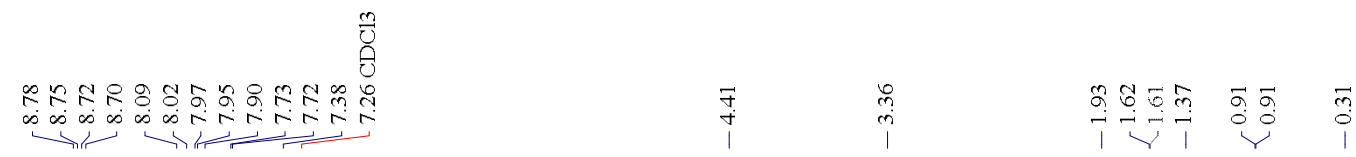
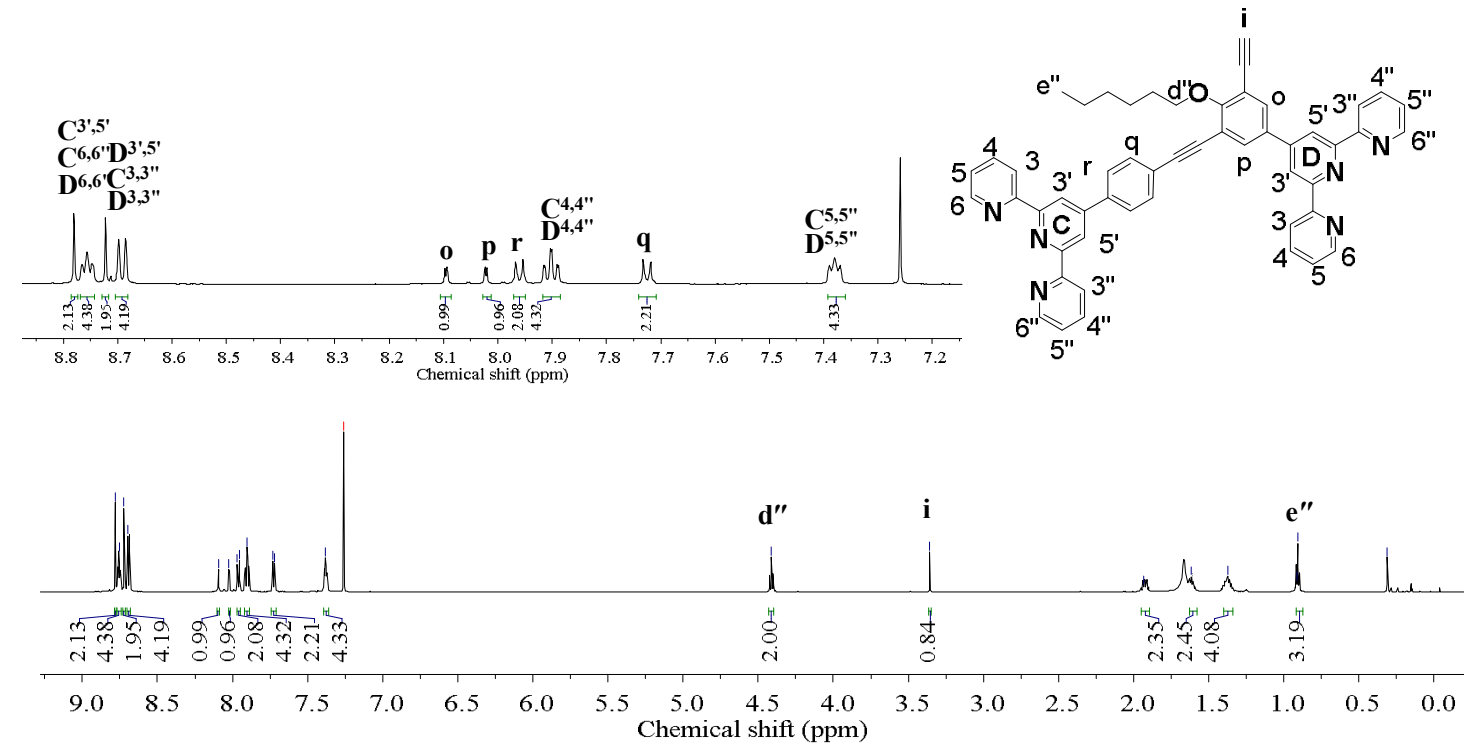

Figure S83. ${ }^{1} \mathrm{H}$ NMR $\left(600 \mathrm{MHz}, \mathrm{CDCl}_{3}\right)$ spectrum of compound 18.

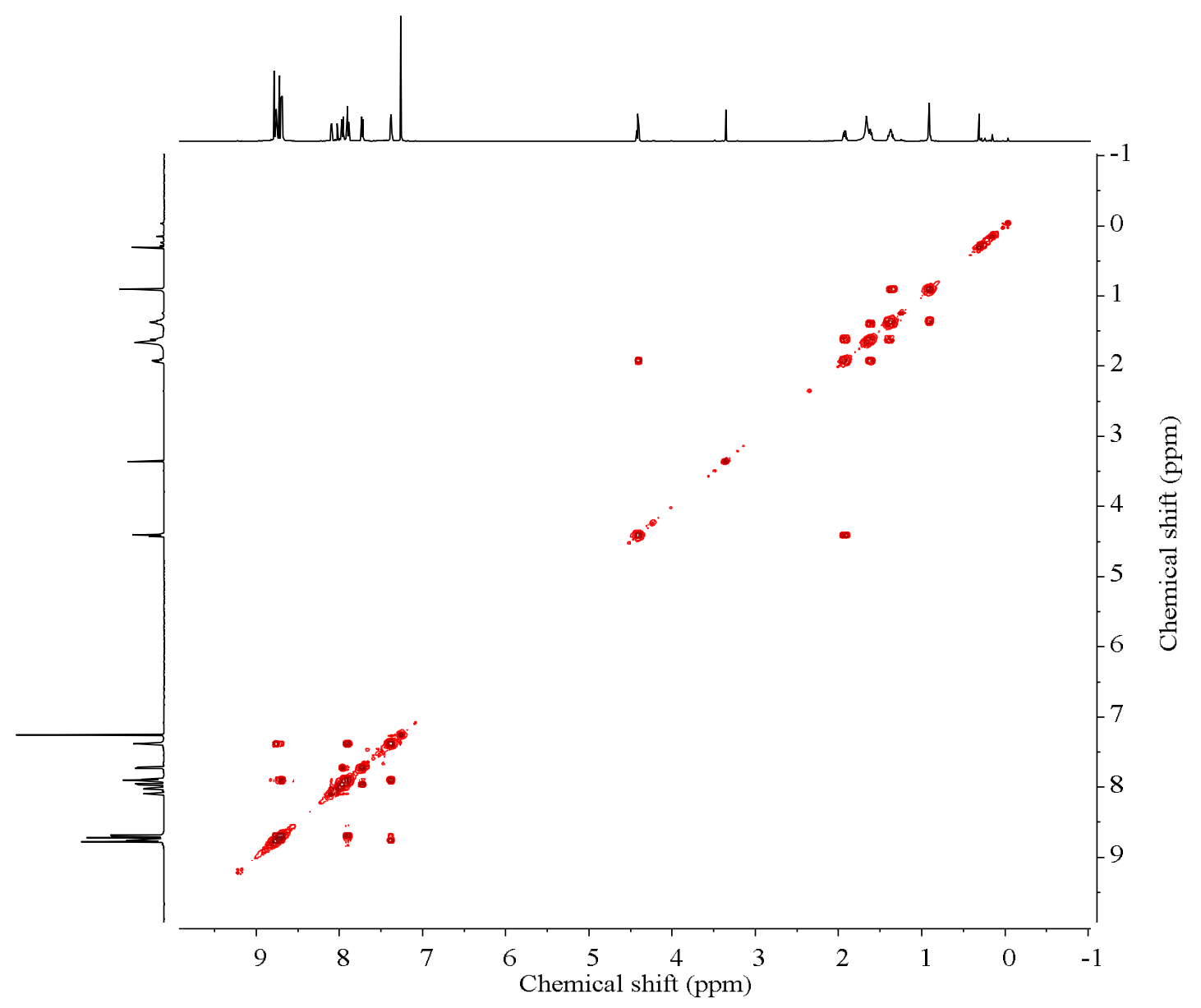

Figure S84. 2D COSY NMR (600 MHz, $\left.\mathrm{CDCl}_{3}\right)$ spectrum of compound 18. 


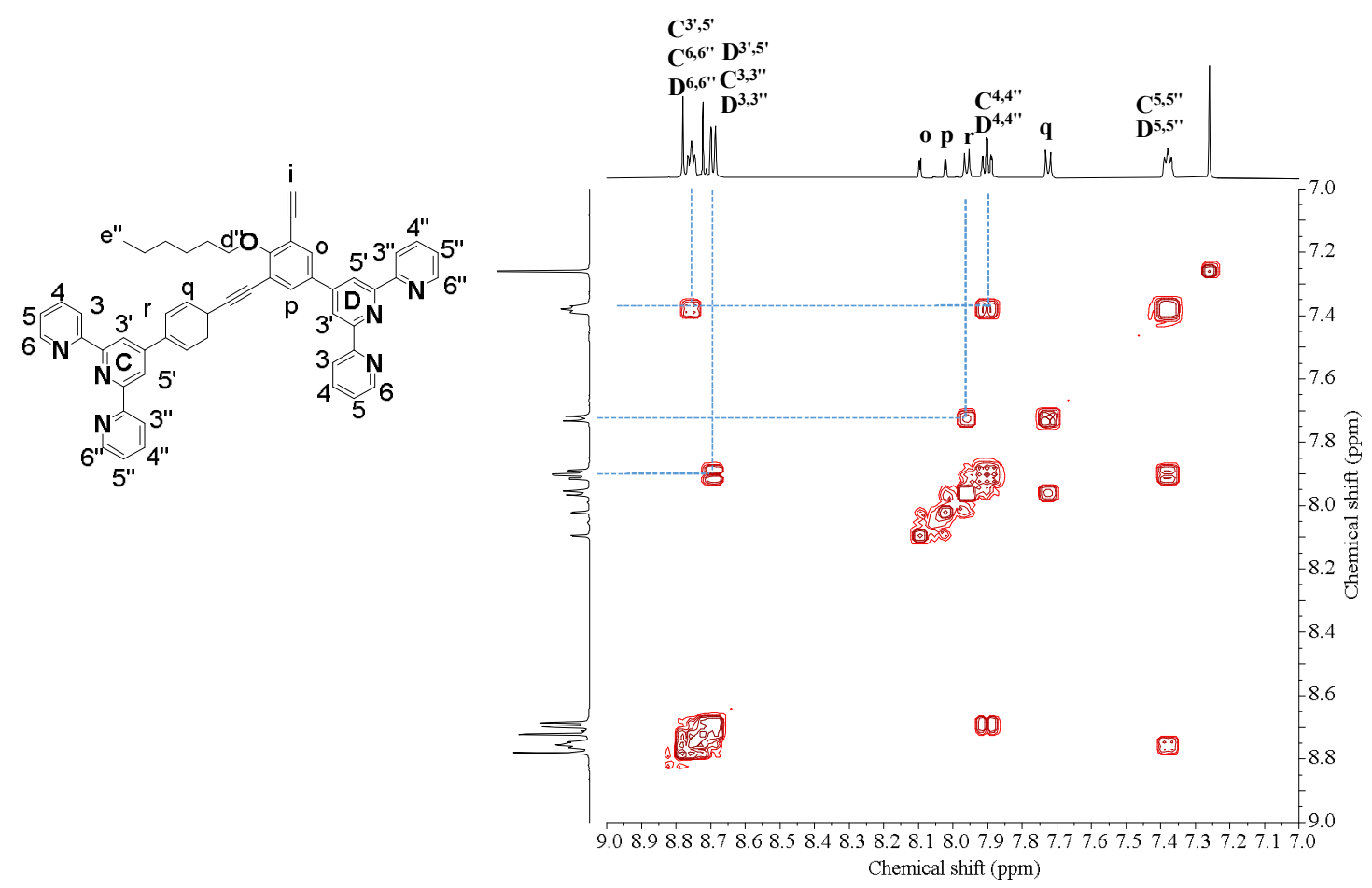

Figure S85. 2D COSY NMR (600 $\left.\mathrm{MHz}, \mathrm{CDCl}_{3}\right)$ spectrum of compound $\mathbf{1 8}$ (aromatic region).

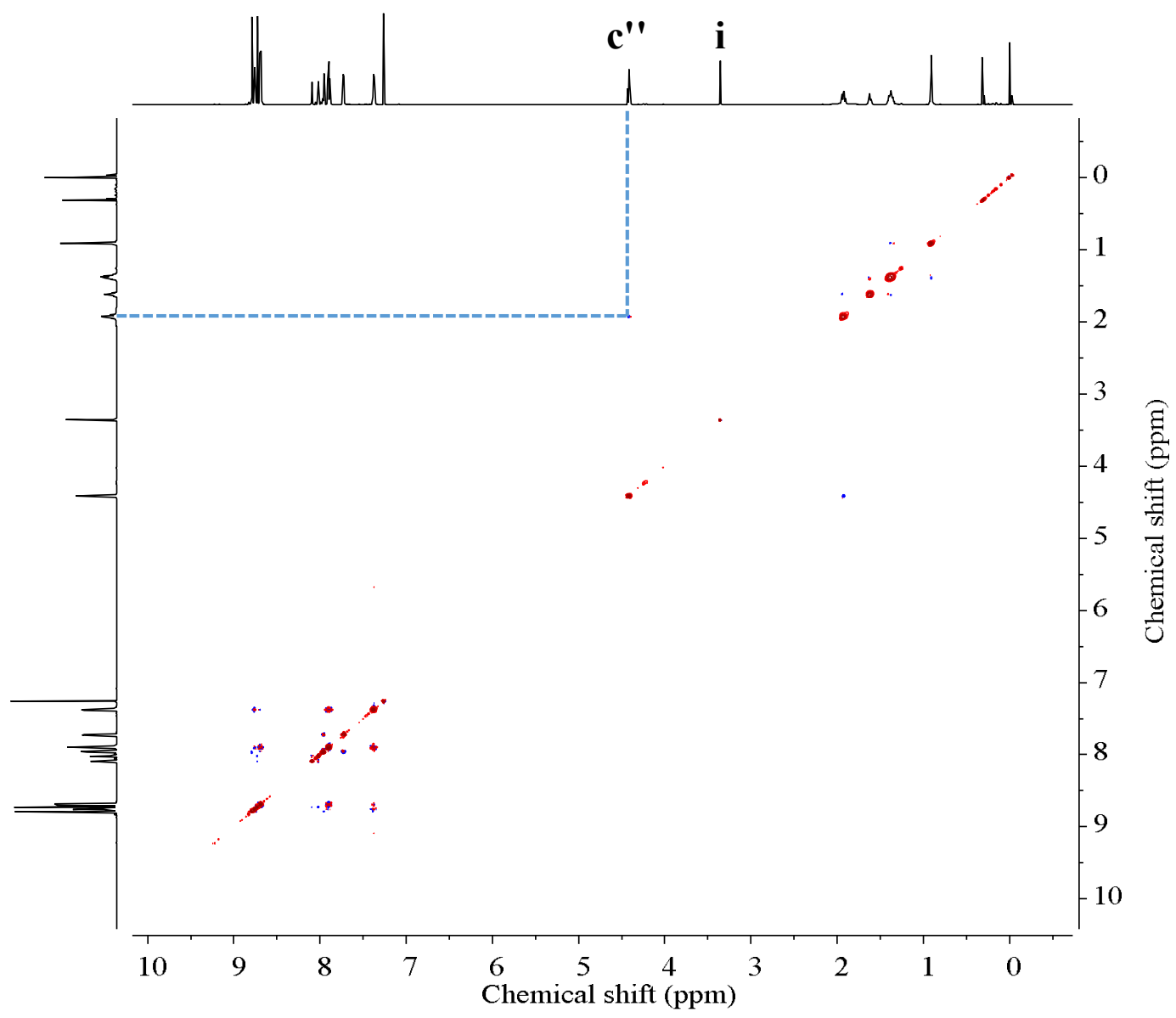

Figure S86. 2D NOESY NMR (600 MHz, $\left.\mathrm{CDCl}_{3}\right)$ spectrum of compound 18. 


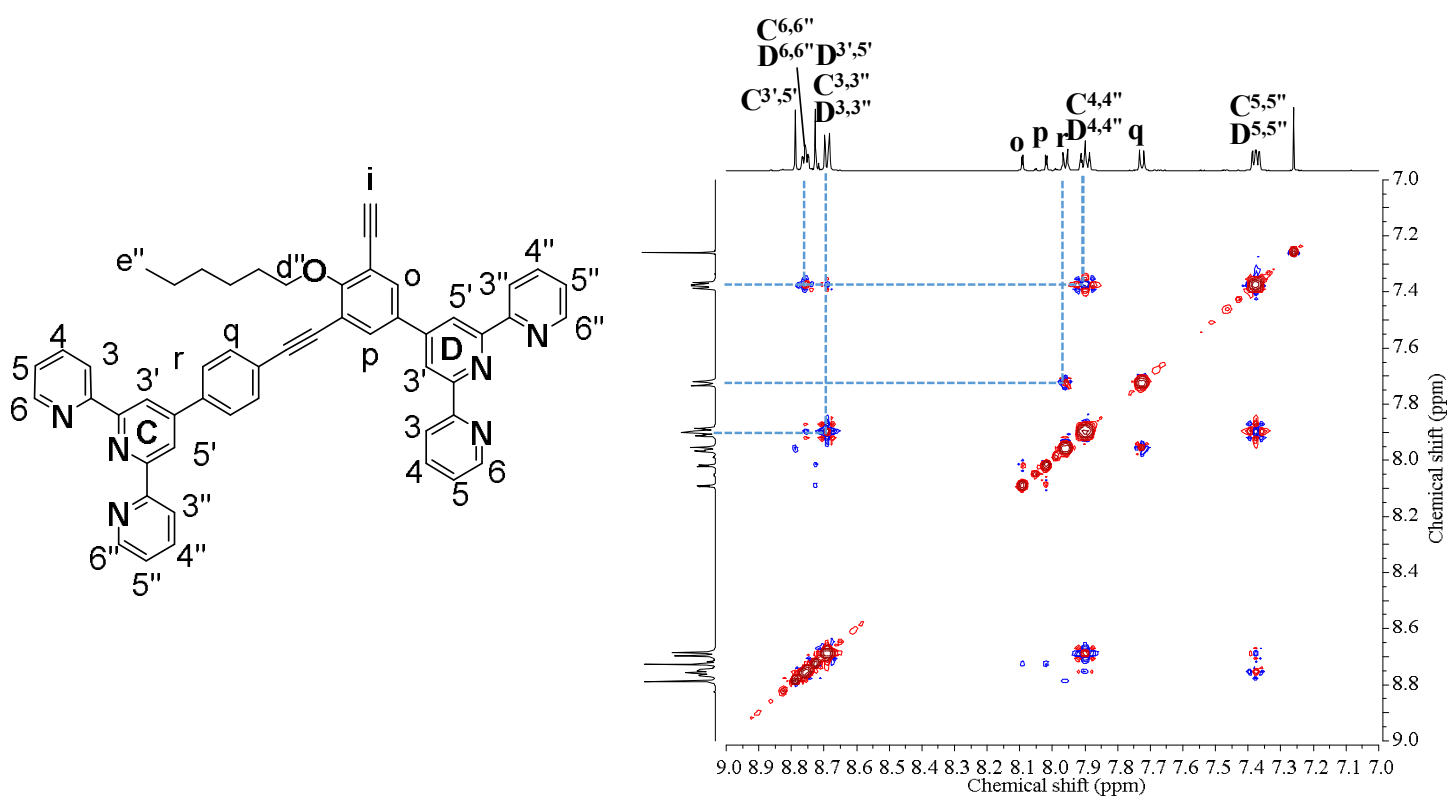

Figure S87. 2D NOESY NMR (600 $\left.\mathrm{MHz}, \mathrm{CDCl}_{3}\right)$ spectrum of compound 18 (aromatic region).
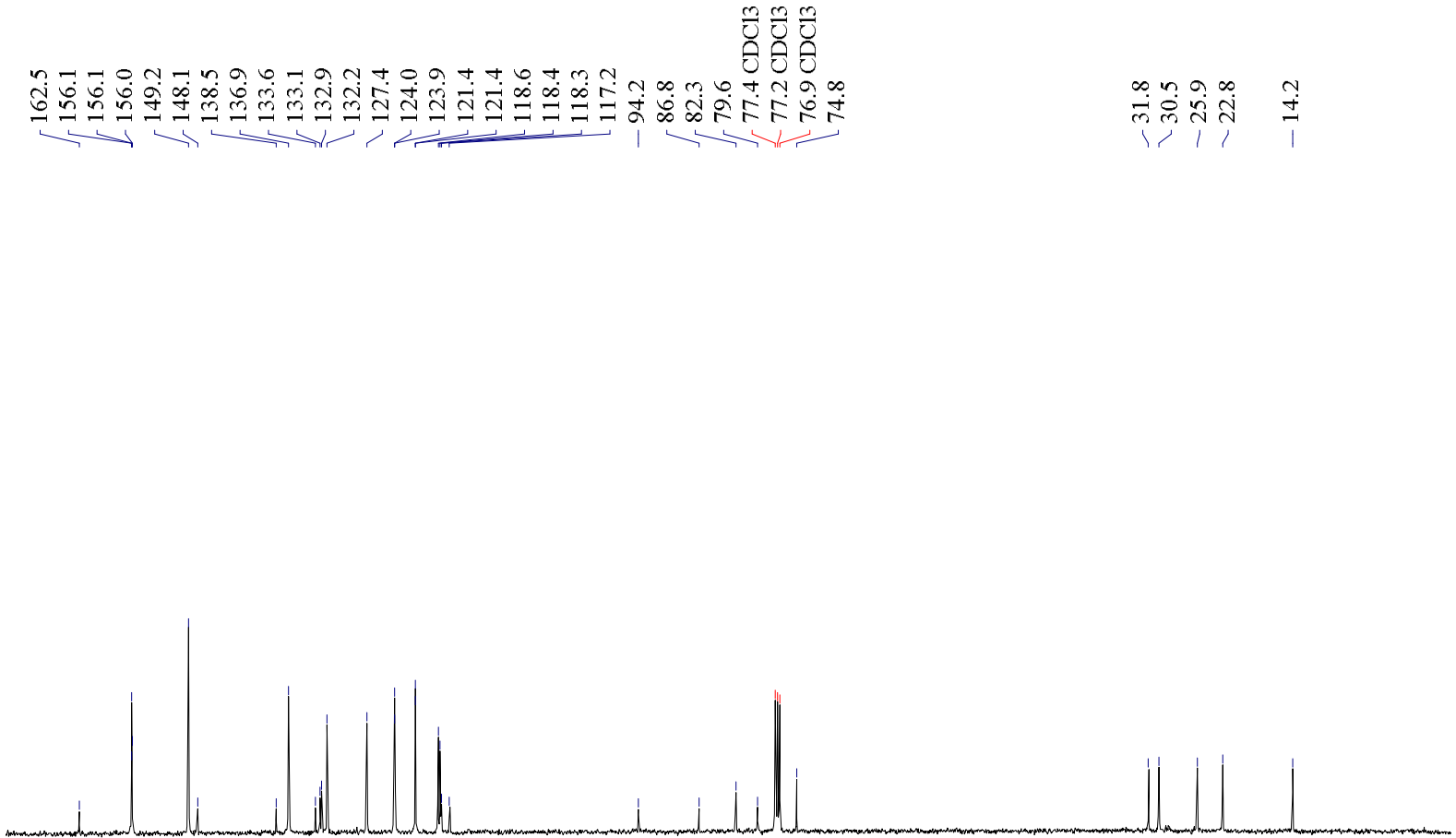

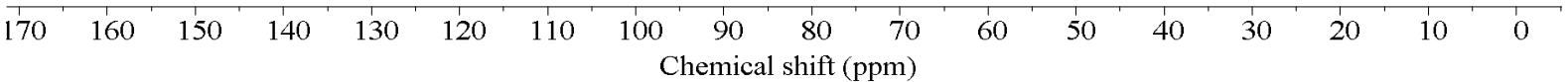

Figure S88. ${ }^{13} \mathrm{C}$ NMR $\left(150 \mathrm{MHz}, \mathrm{CDCl}_{3}\right)$ spectrum of compound 18. 

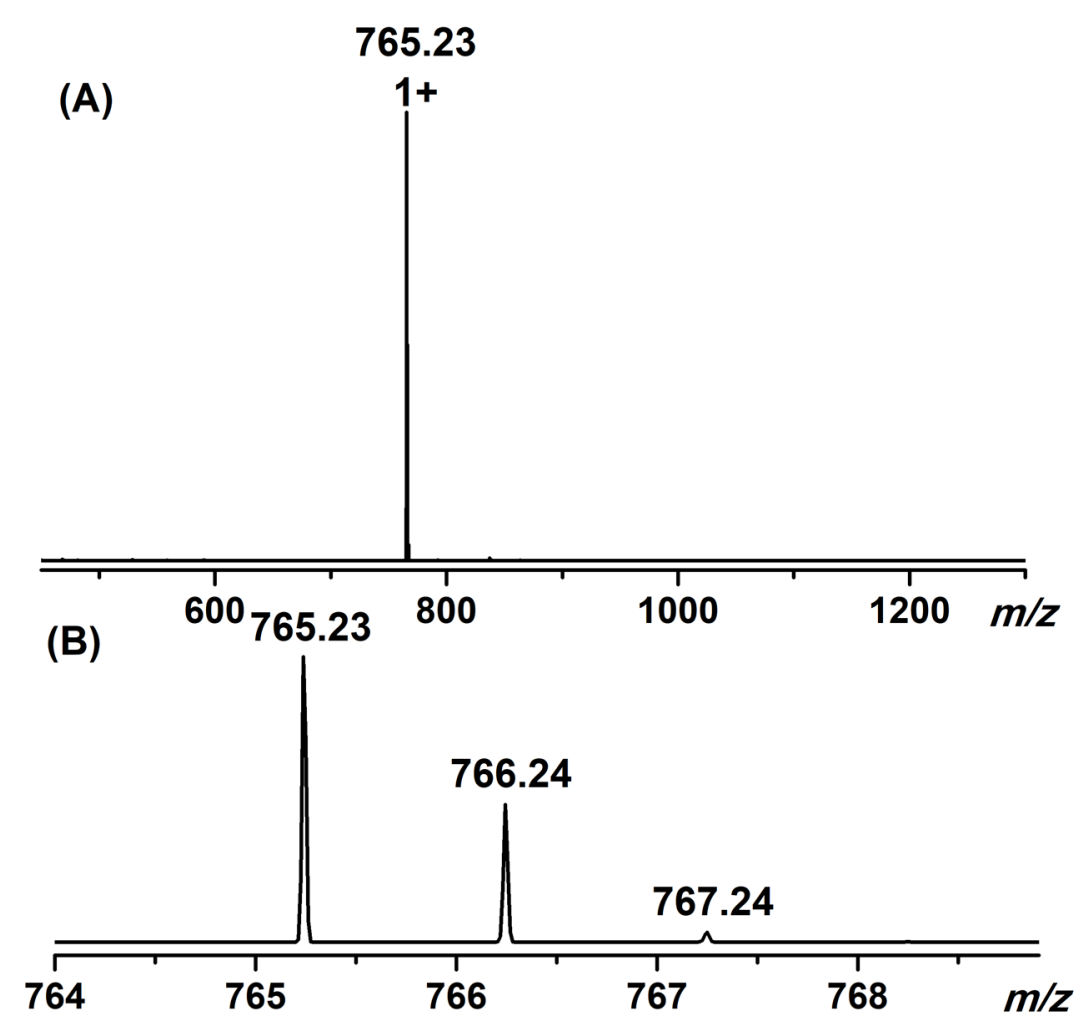

Figure S89. (A) ESI-MS spectrum of compound 18 in $\mathrm{CHCl}_{3} / \mathrm{CH}_{3} \mathrm{OH}(1 / 3)$ and (B) isotope pattern.

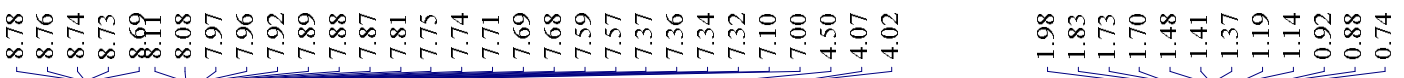

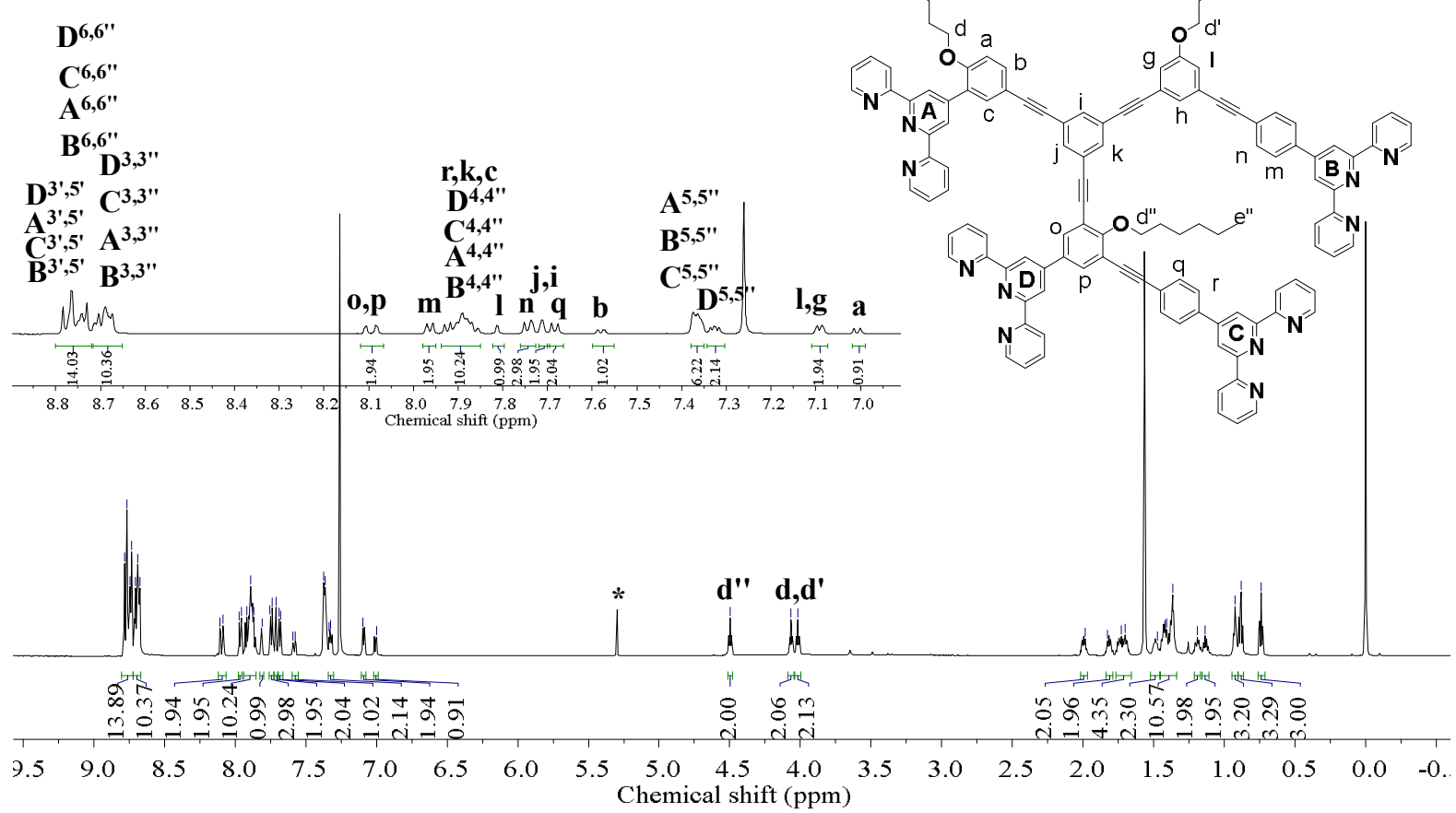

Figure S90. ${ }^{1} \mathrm{H}$ NMR $\left(600 \mathrm{MHz}, \mathrm{CDCl}_{3}\right)$ spectrum of ligand $\mathbf{L A}\left({ }^{*}-\mathrm{CH}_{2} \mathrm{Cl}_{2}\right)$. 


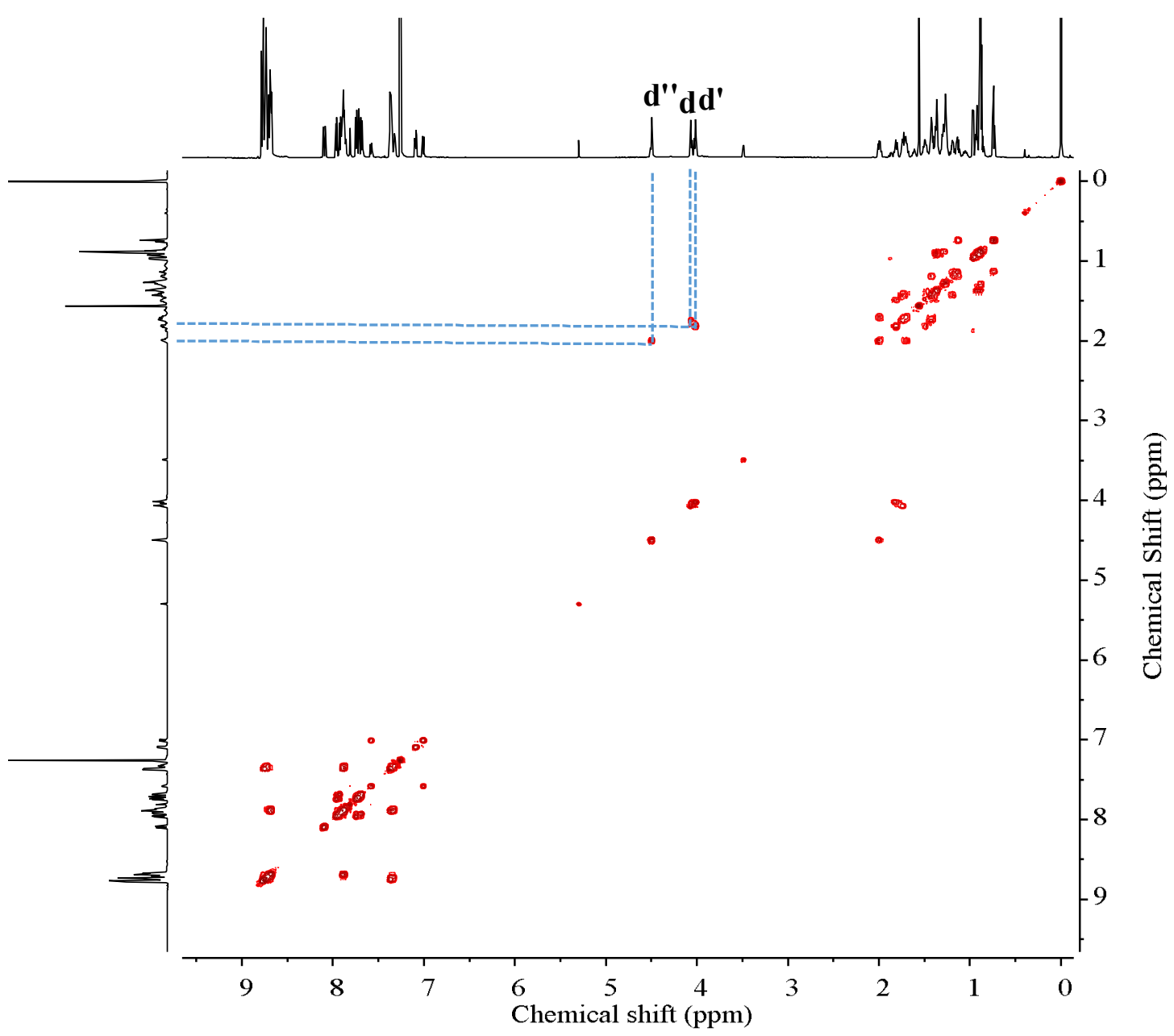

Figure S91. 2D COSY NMR (600 MHz, $\left.\mathrm{CDCl}_{3}\right)$ spectrum of ligand $\mathbf{L A}$.

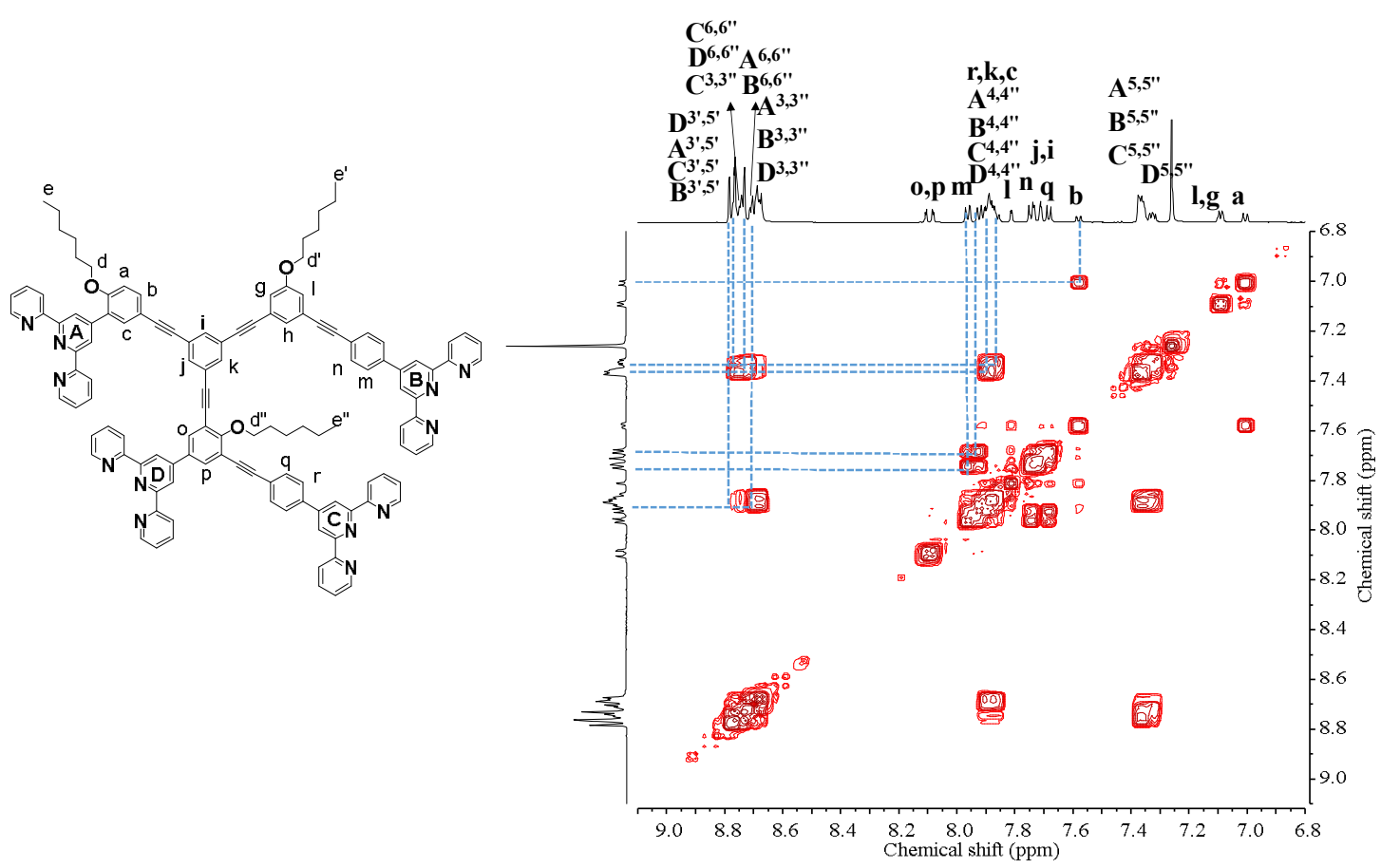

Figure S92. 2D COSY NMR (600 MHz, $\mathrm{CDCl}_{3}$ ) spectrum of ligand $\mathbf{L A}$ (aromatic region). 


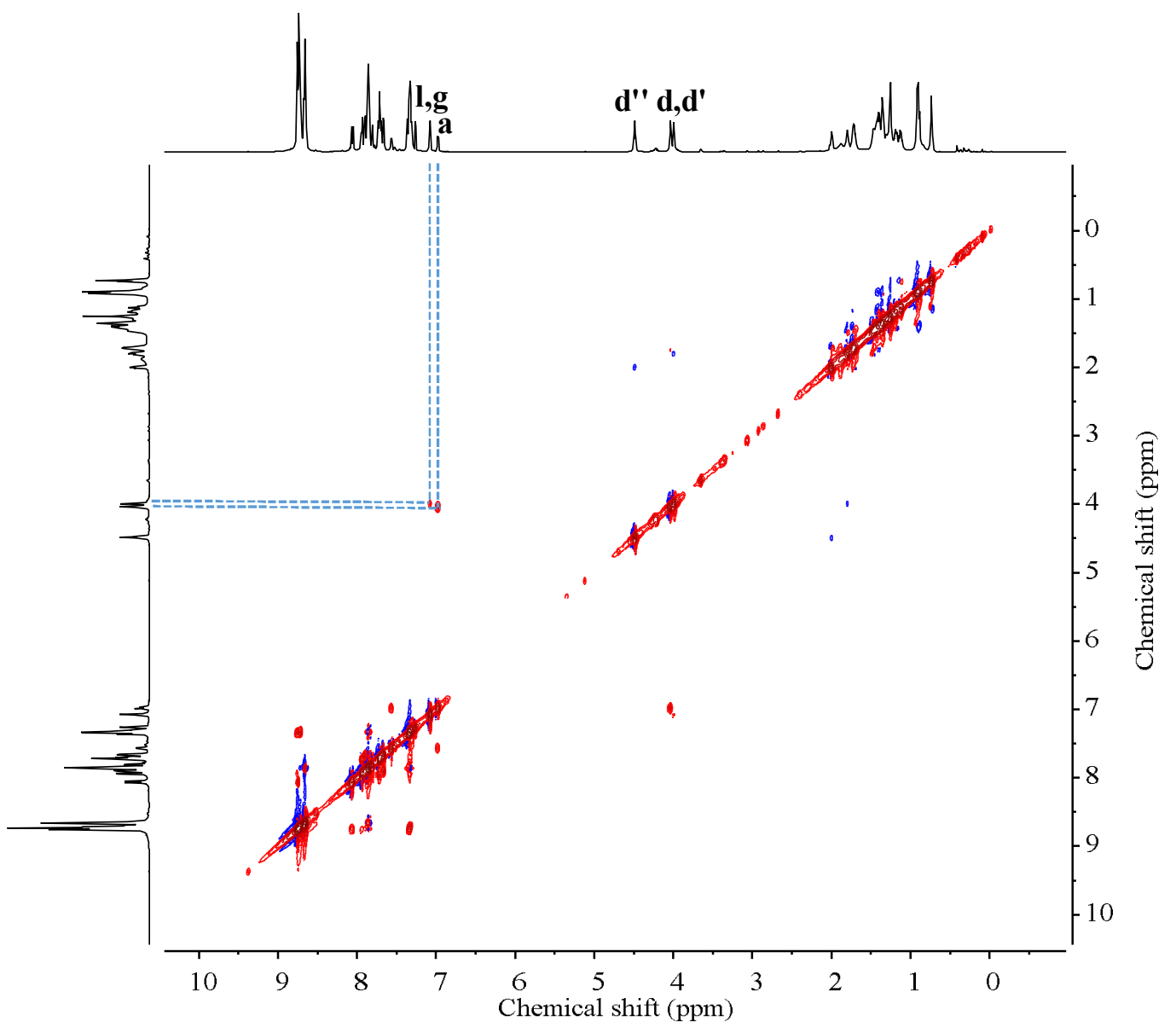

Figure S93. 2D NOESY NMR (600 MHz, $\left.\mathrm{CDCl}_{3}\right)$ spectrum of ligand $\mathbf{L A}$.

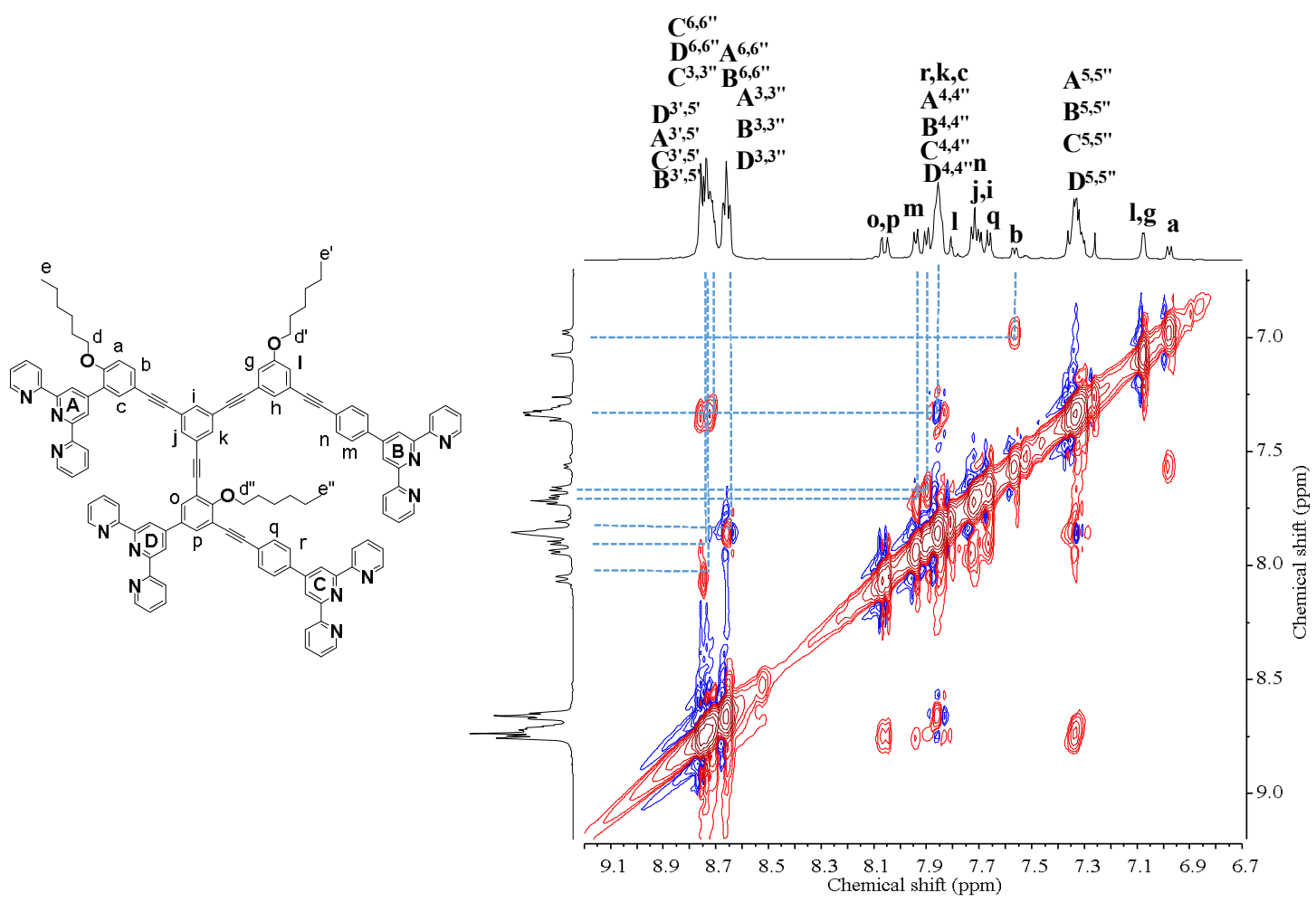

Figure S94. 2D NOESY NMR (600 MHz, $\mathrm{CDCl}_{3}$ ) spectrum of ligand $\mathbf{L A}$ (aromatic region). 

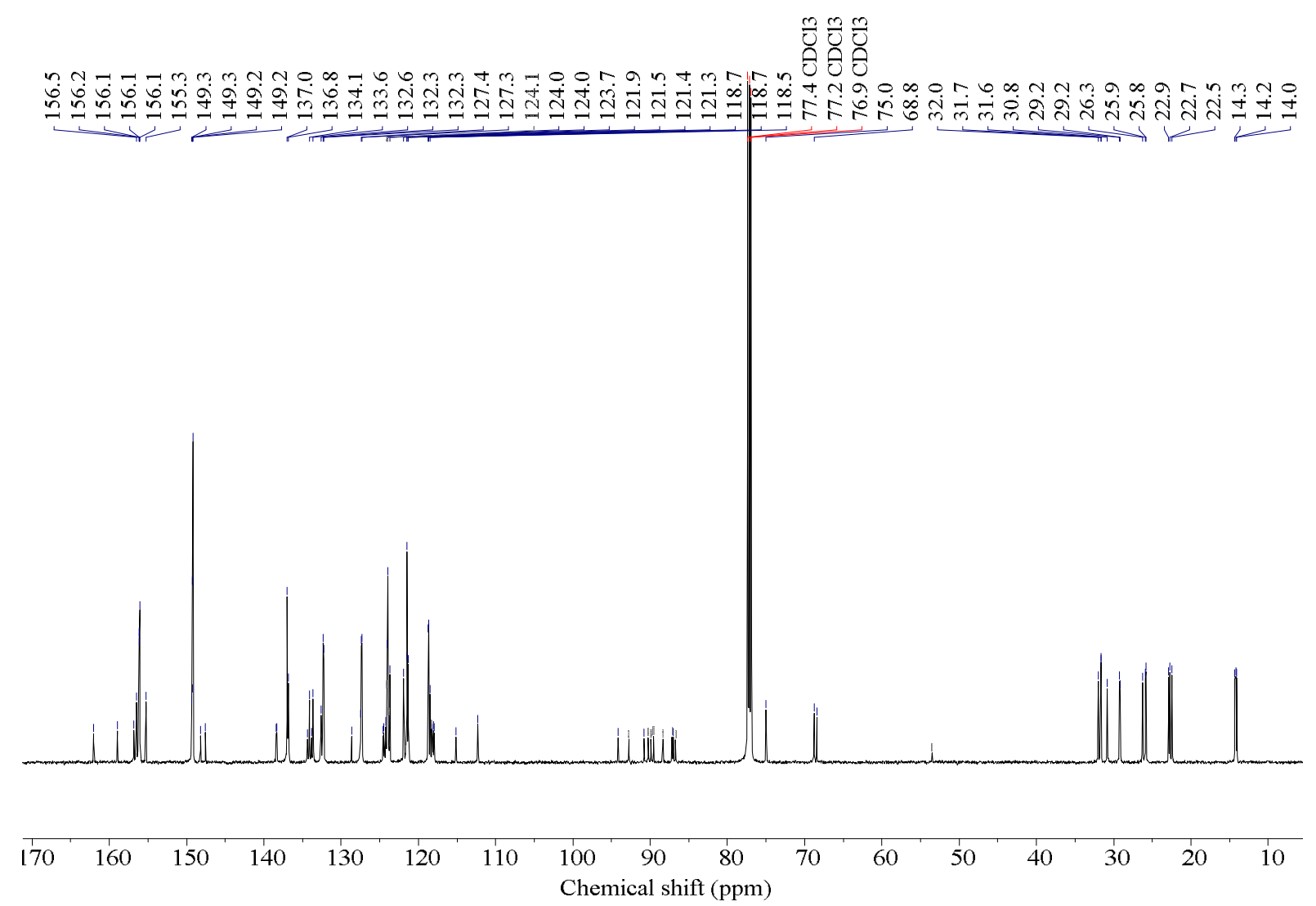

Figure S95. ${ }^{13} \mathrm{C}$ NMR $\left(150 \mathrm{MHz}, \mathrm{CDCl}_{3}\right)$ spectrum of ligand $\mathbf{L A}$.

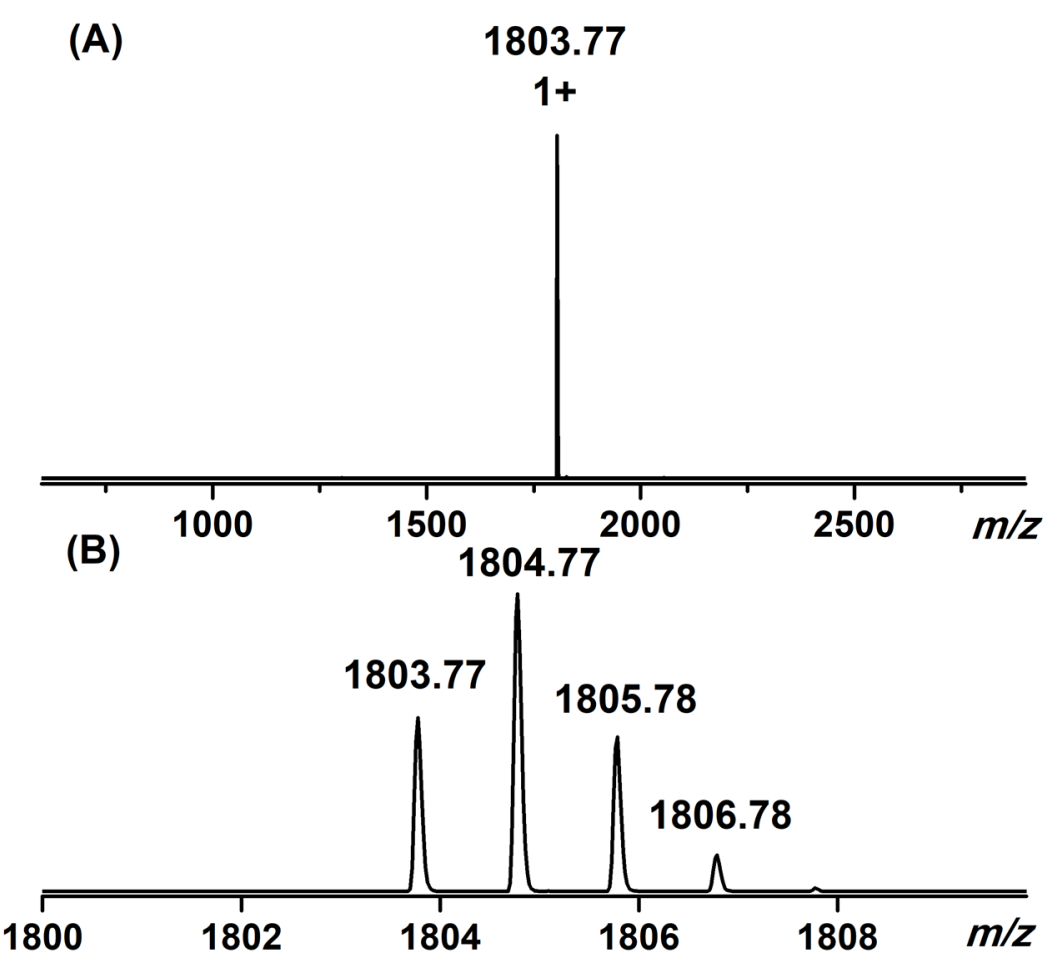

Figure S96. (A) MALDI-TOF spectrum of ligand $\mathbf{L A}$ in $\mathrm{CHCl}_{3} / \mathrm{CH}_{3} \mathrm{OH}$ (1/3) and (B) isotope pattern. 


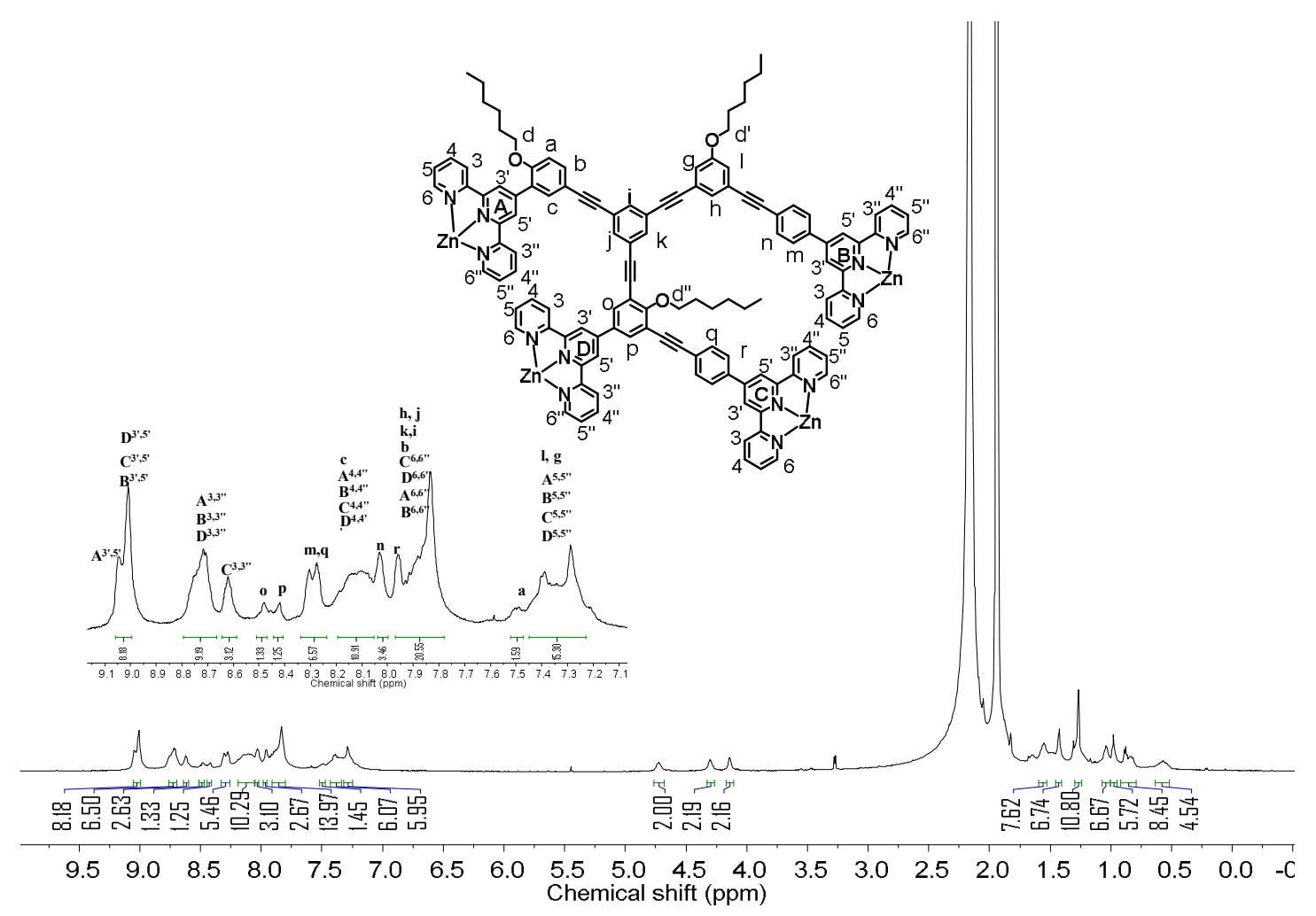

Figure S97. ${ }^{1} \mathrm{H}$ NMR $\left(600 \mathrm{MHz}, \mathrm{CD}_{3} \mathrm{CN}\right)$ spectrum of complexes A.

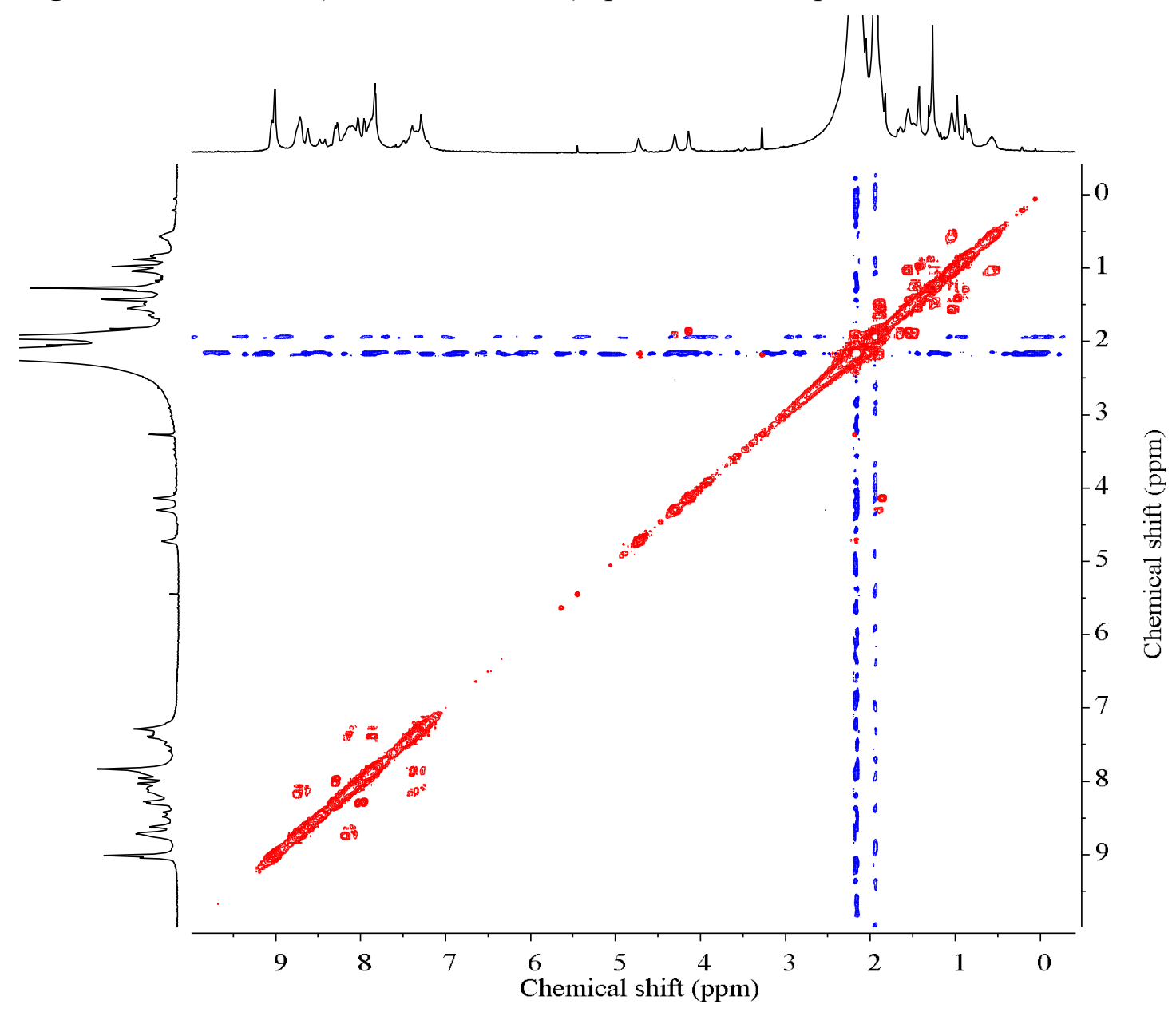

Figure S98. 2D COSY NMR (600 MHz, $\left.\mathrm{CD}_{3} \mathrm{CN}\right)$ spectrum of complexes $\mathbf{A}$. 


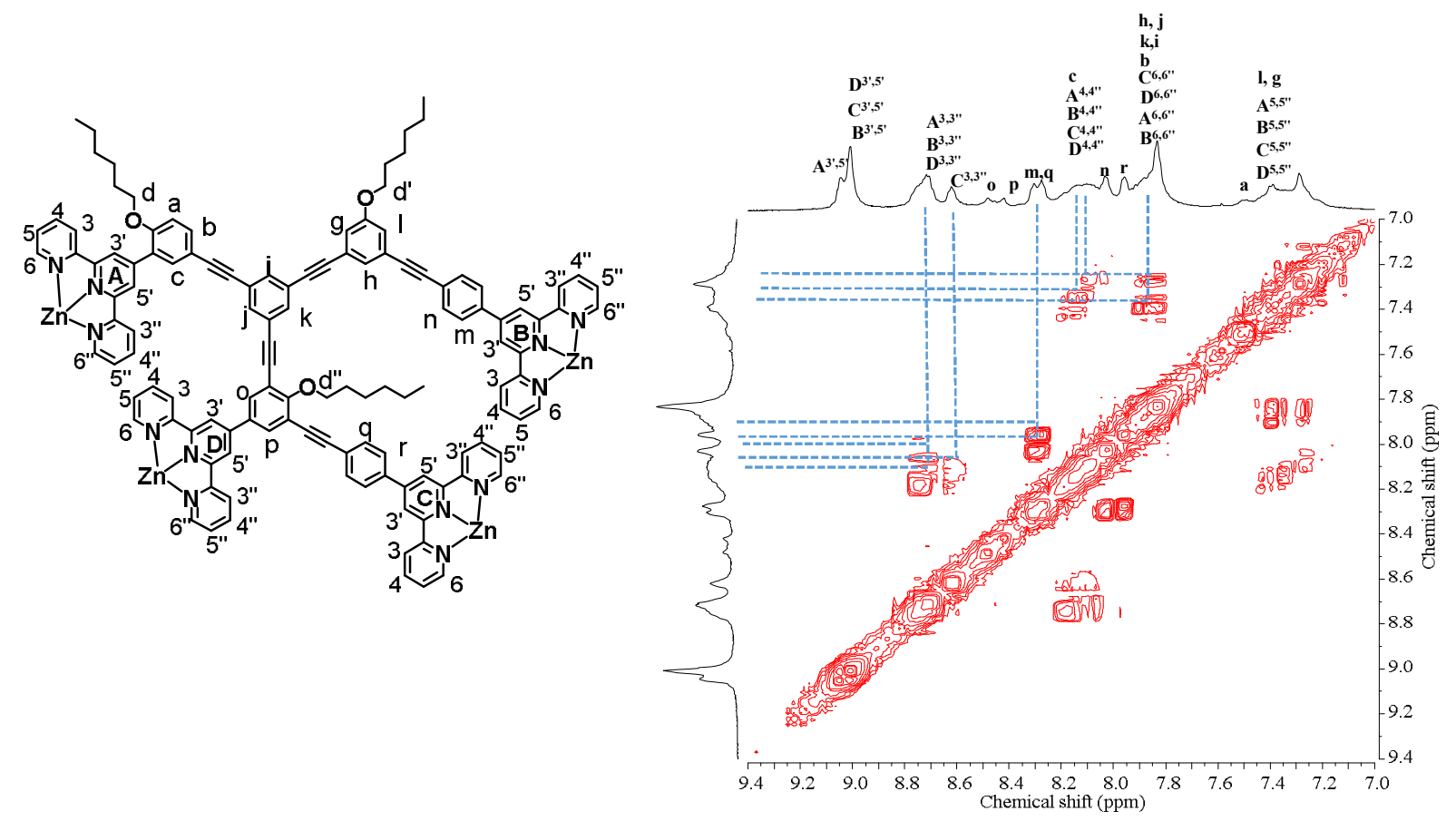

Figure S99. 2D COSY NMR (600 MHz, $\left.\mathrm{CD}_{3} \mathrm{CN}\right)$ spectrum of complexes A (aromatic region).

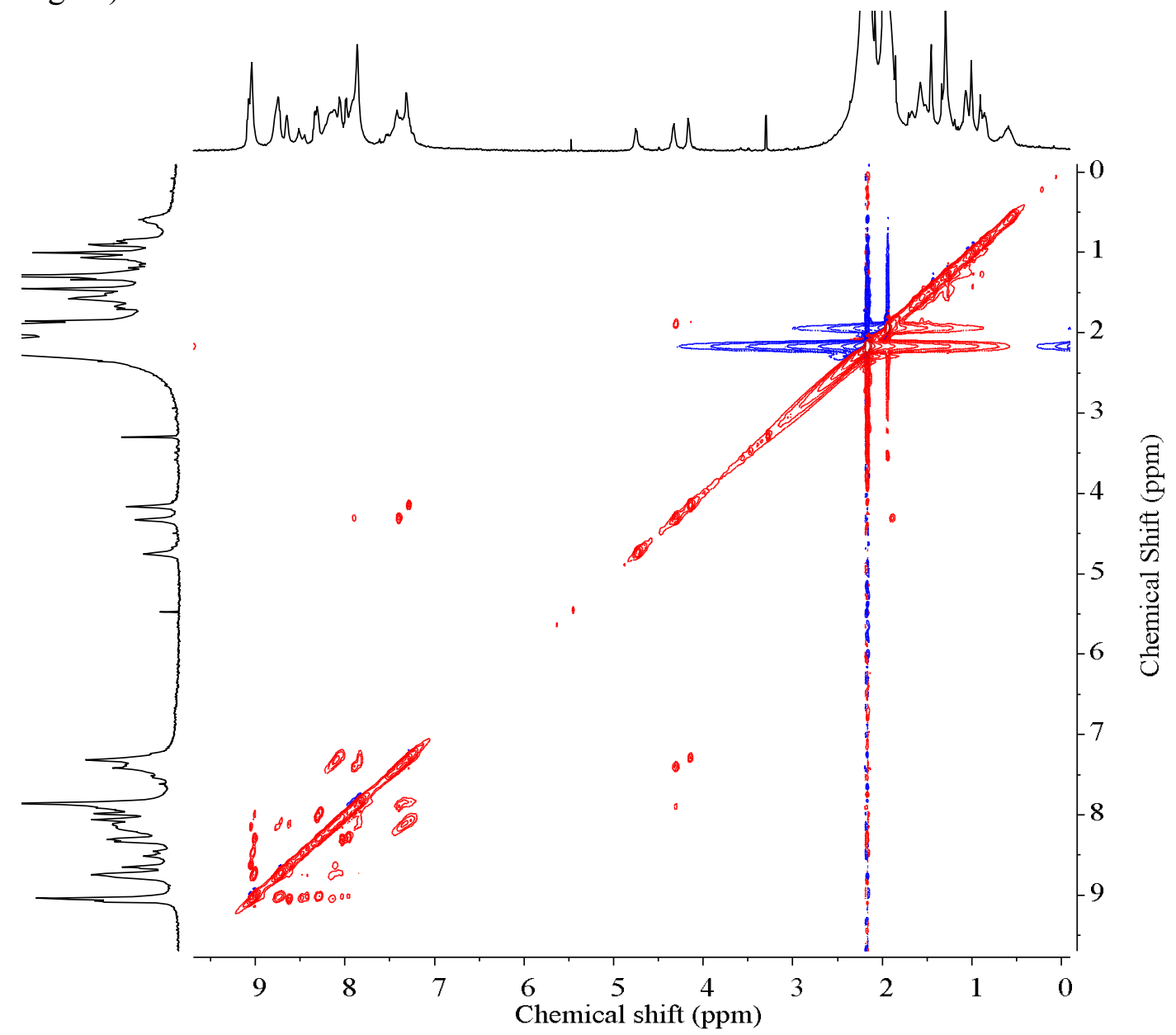

Figure S100. 2D NOESY NMR (600 MHz, $\left.\mathrm{CD}_{3} \mathrm{CN}\right)$ spectrum of complexes A. 


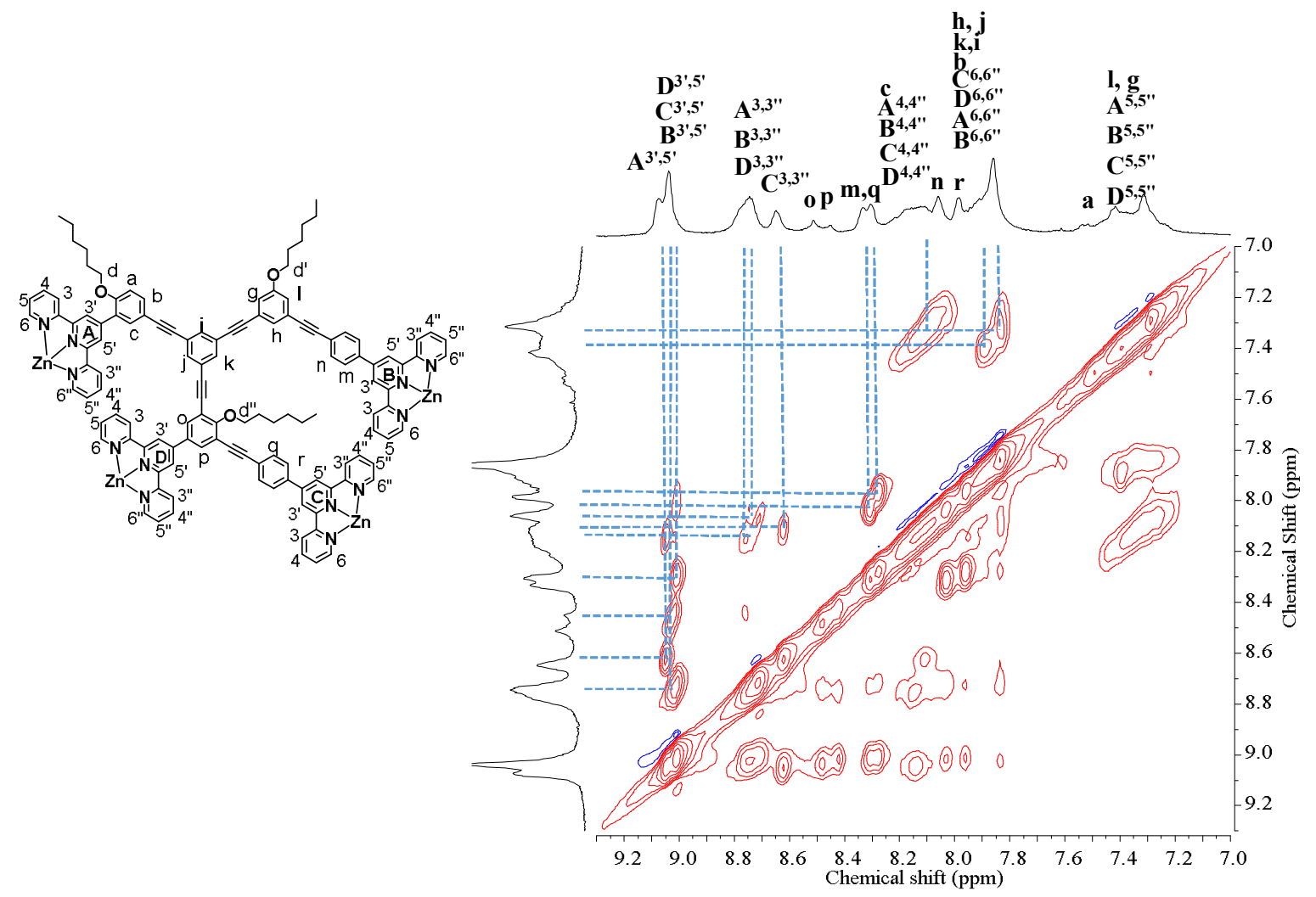

Figure S101. 2D NOESY NMR (600 MHz, $\left.\mathrm{CD}_{3} \mathrm{CN}\right)$ spectrum of complexes A (aromatic region).

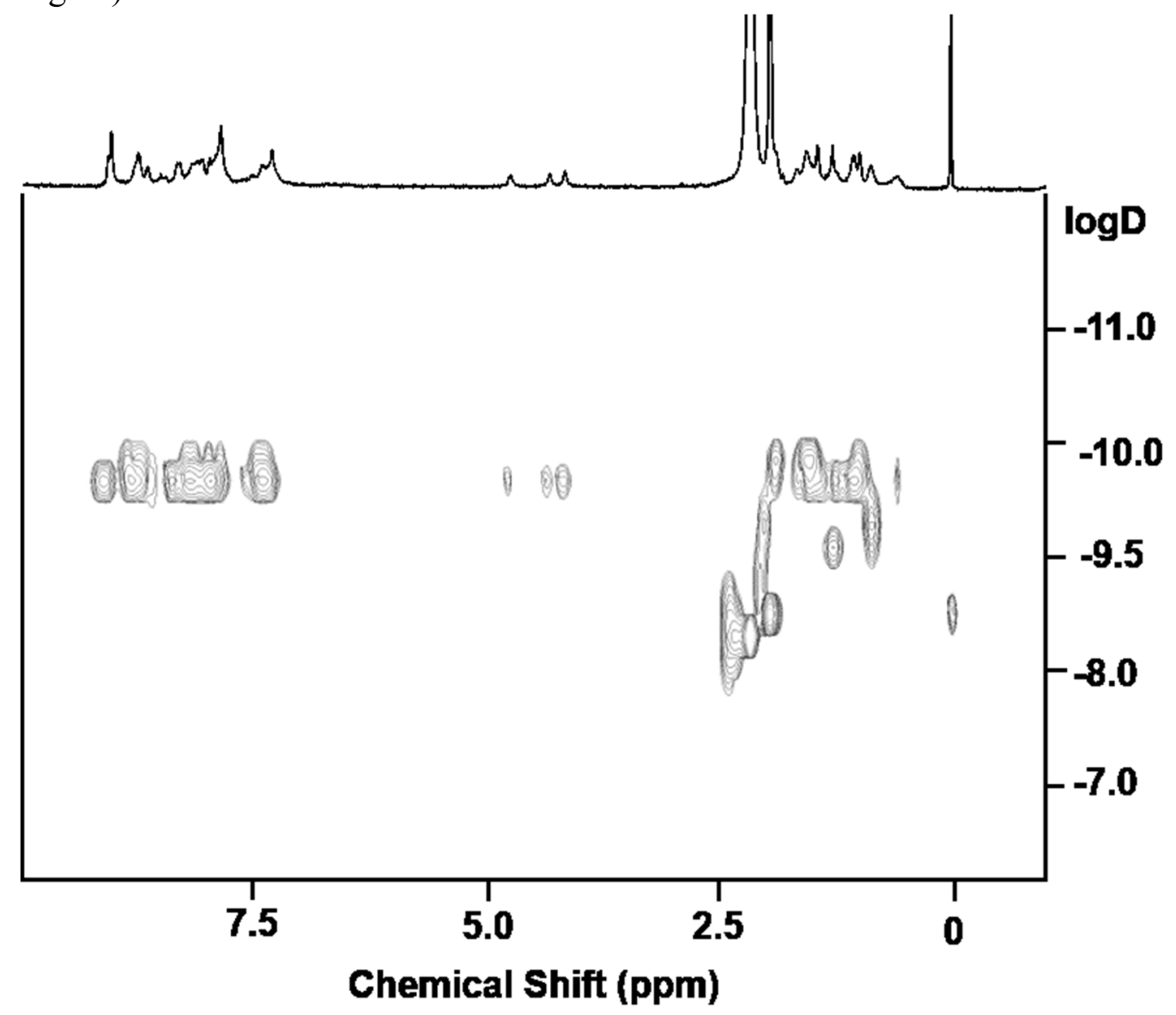


Figure S102. DOSY NMR (500 MHz, $\left.\mathrm{CD}_{3} \mathrm{CN}\right)$ spectrum of complexes $\mathbf{A}$.

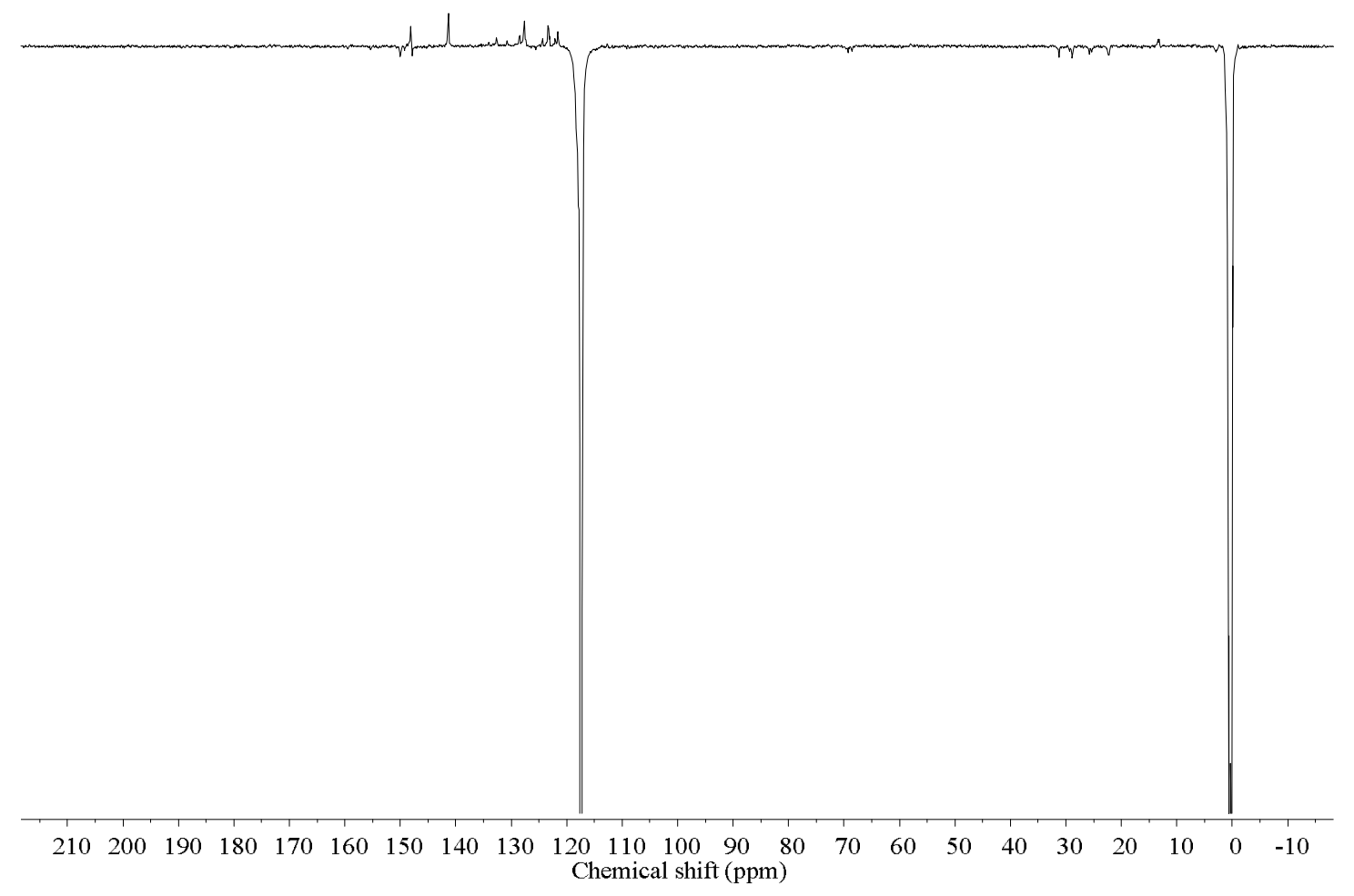

Figure S103. DEPTQ ${ }^{13} \mathrm{C}$ NMR $\left(150 \mathrm{MHz}, \mathrm{CD}_{3} \mathrm{CN}\right)$ spectrum of complexes A. 
$15+$

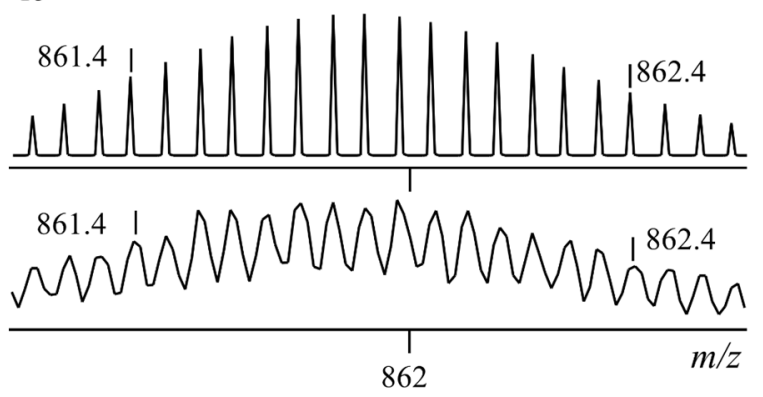

$17+$

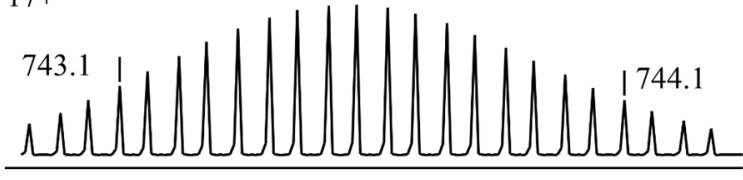

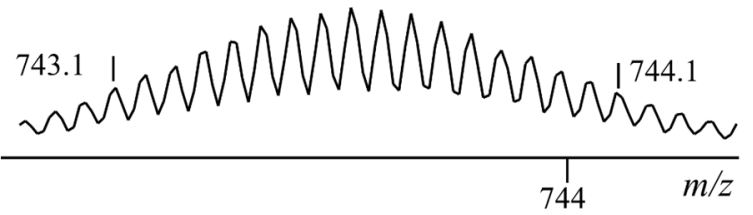

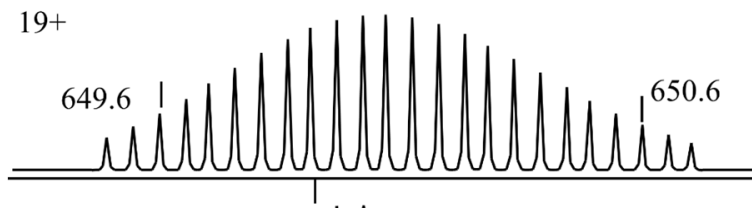

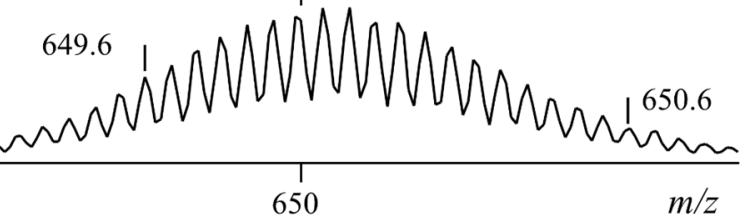

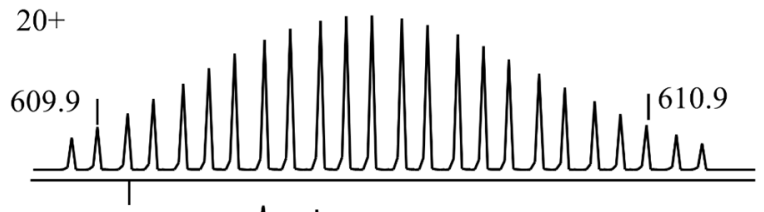
$\underbrace{209.9}_{610}$

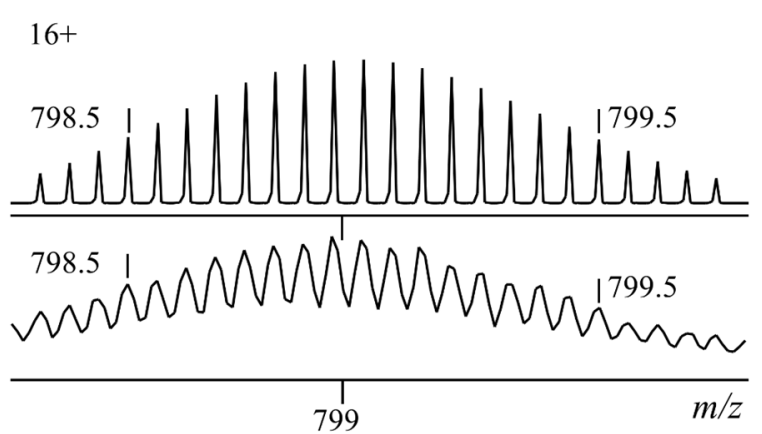

$18+$

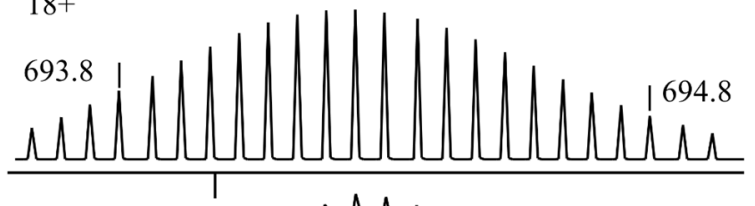
$\underbrace{293.8}_{693}$

$20+$

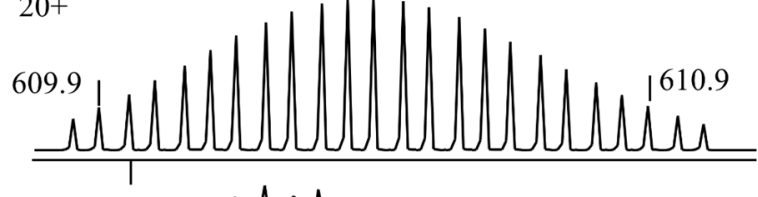
$\underbrace{209.9}_{610}$

$22+$

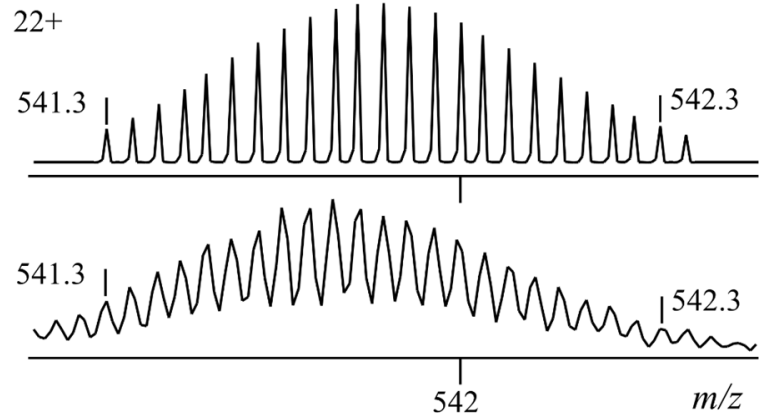

Figure S104. Measured (bottom) and calculated (top) isotope patterns for different charge states observed from $\mathbf{A}\left(\mathrm{PF}_{6}{ }^{-}\right.$as counterion). 


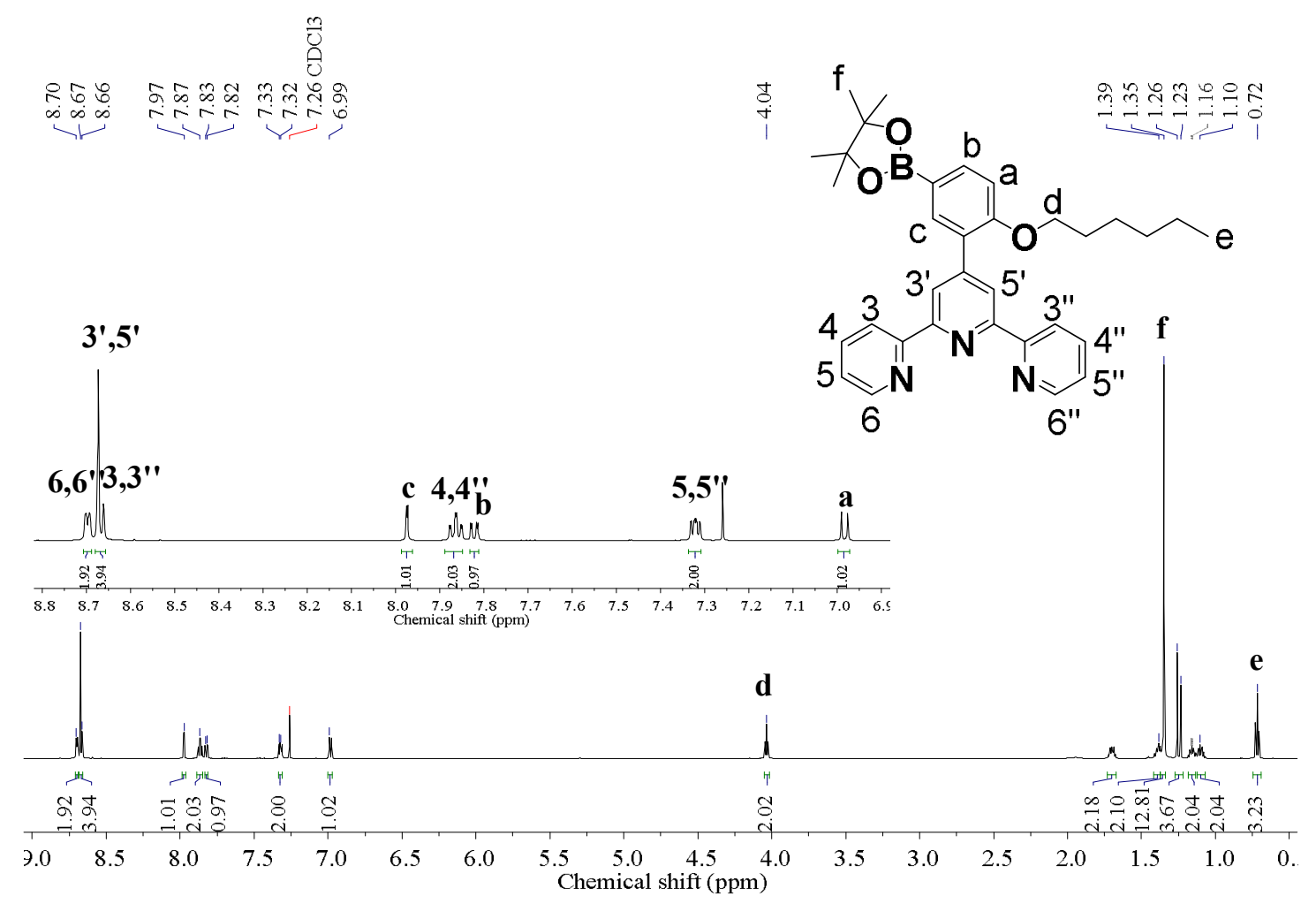

Figure S105. ${ }^{1} \mathrm{H}$ NMR $\left(600 \mathrm{MHz}, \mathrm{CDCl}_{3}\right)$ spectrum of compound 19.

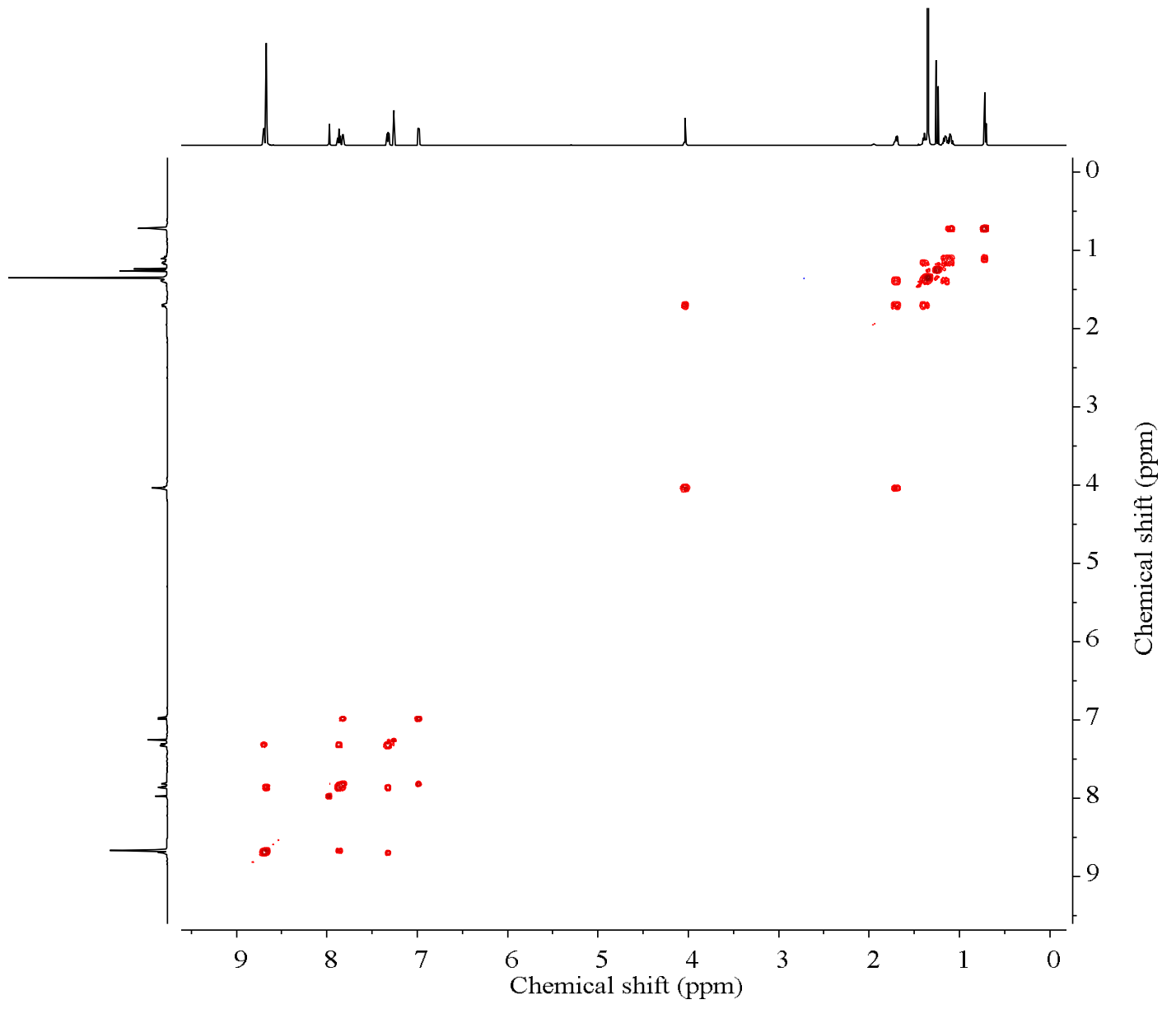

Figure S106. 2D COSY NMR (600 MHz, $\left.\mathrm{CDCl}_{3}\right)$ spectrum of compound 19. 


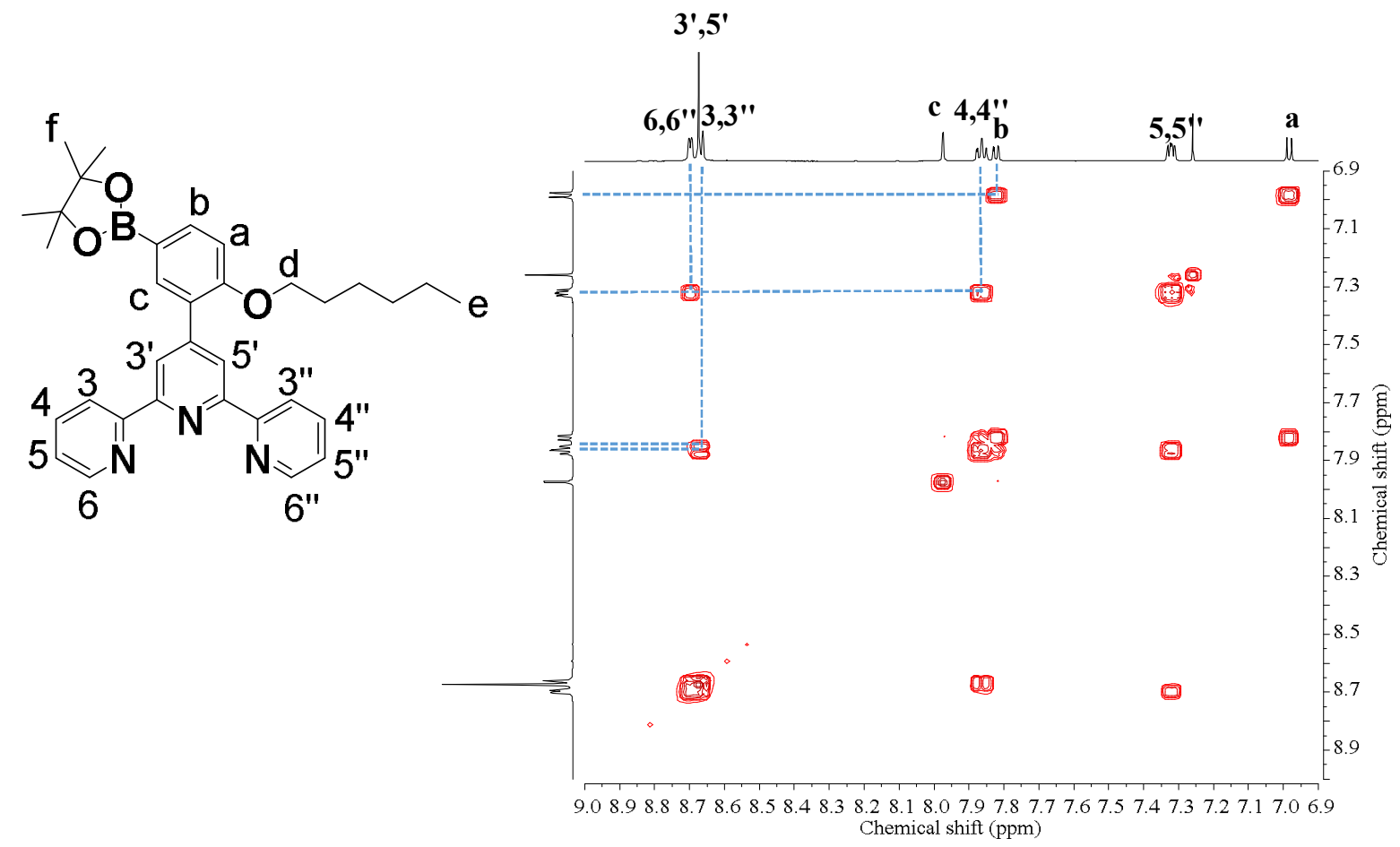

Figure S107. 2D COSY NMR (600 $\left.\mathrm{MHz}, \mathrm{CDCl}_{3}\right)$ spectrum of compound 19 (aromatic region).
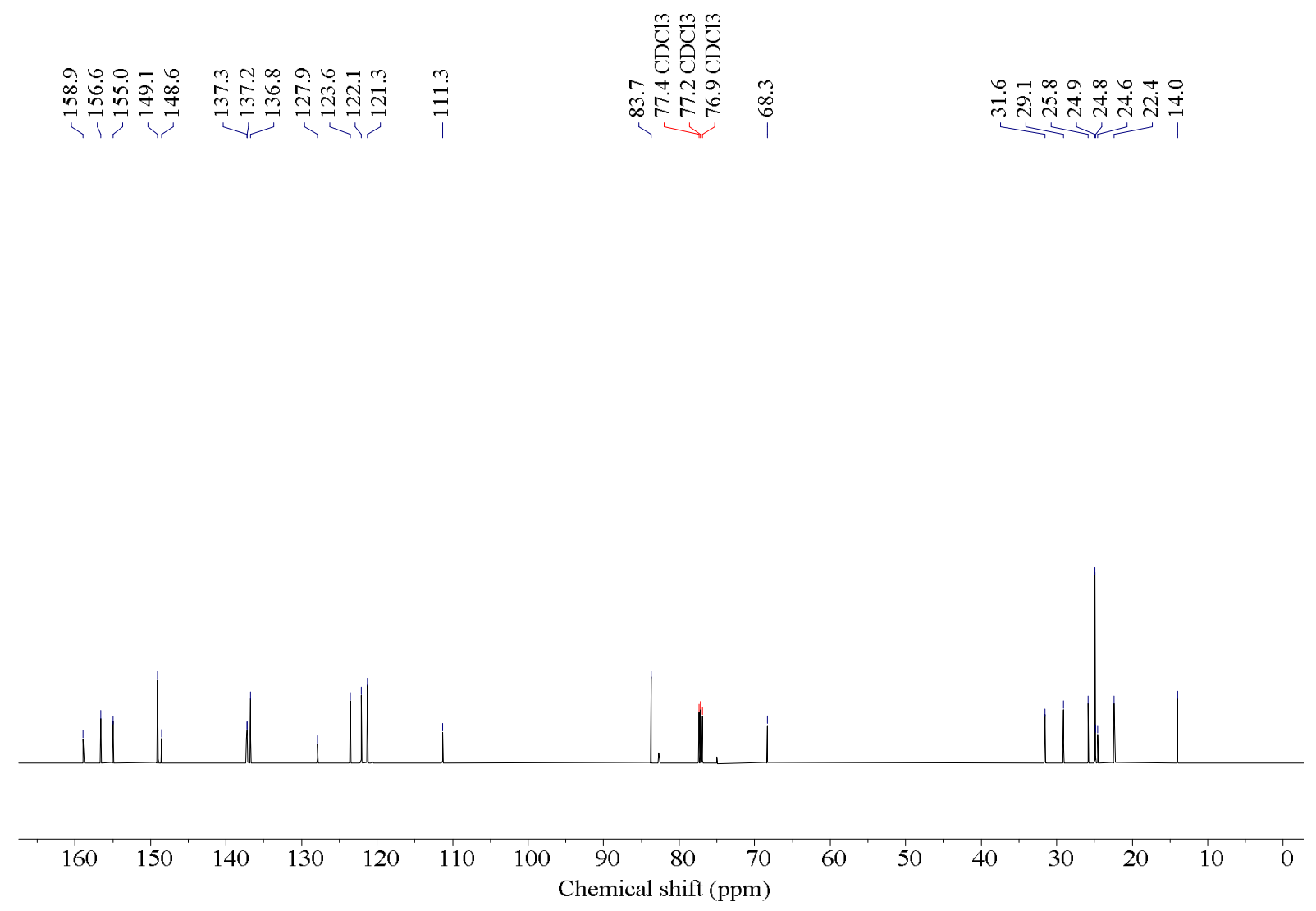

Figure S108. ${ }^{13} \mathrm{C}$ NMR $\left(150 \mathrm{MHz}, \mathrm{CDCl}_{3}\right)$ spectrum of compound 19. 


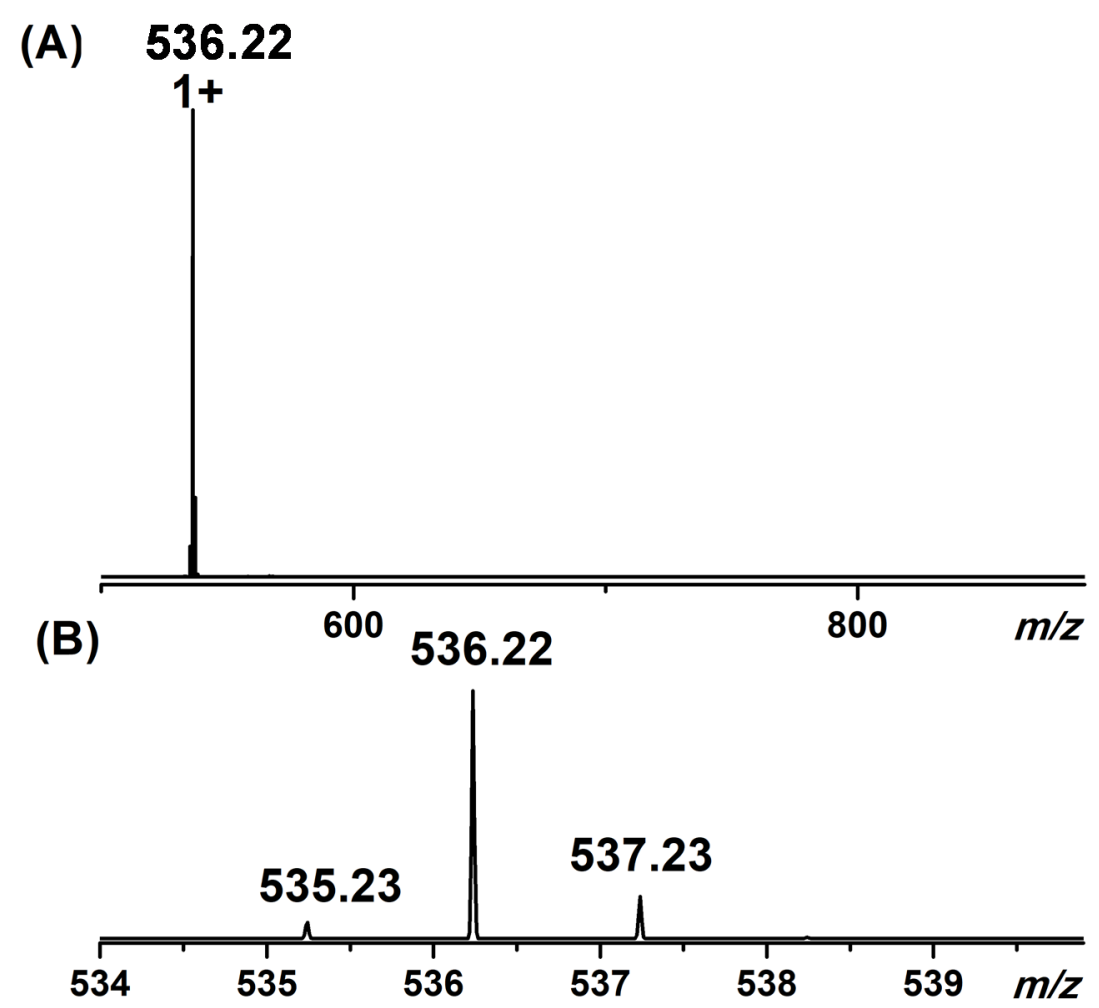

Figure S109. (A) ESI-MS spectrum of compound 19 in $\mathrm{CHCl}_{3} / \mathrm{CH}_{3} \mathrm{OH}(1 / 3)$ and (B) isotope pattern.
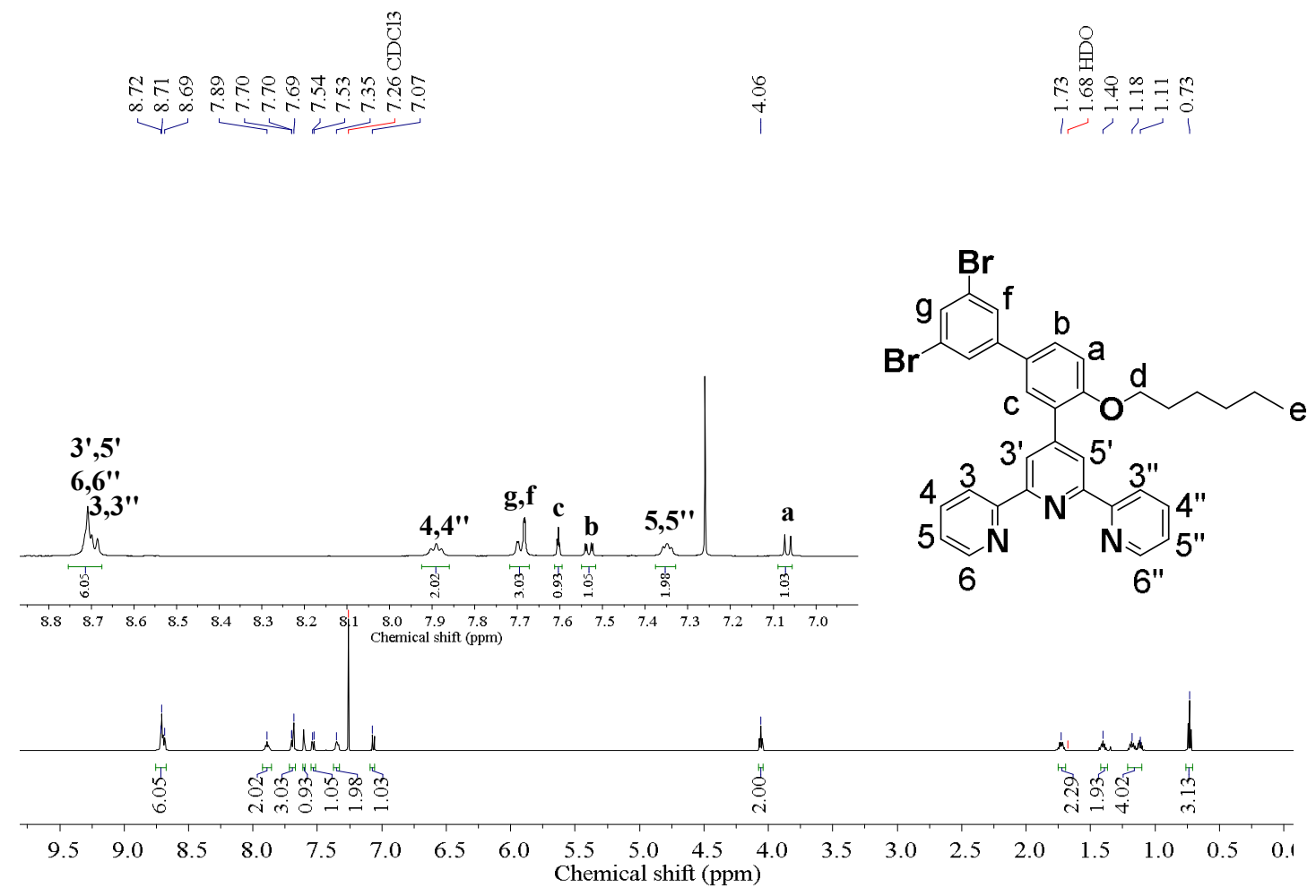

Figure S110. ${ }^{1} \mathrm{H}$ NMR $\left(600 \mathrm{MHz}, \mathrm{CDCl}_{3}\right)$ spectrum of compound 20. 


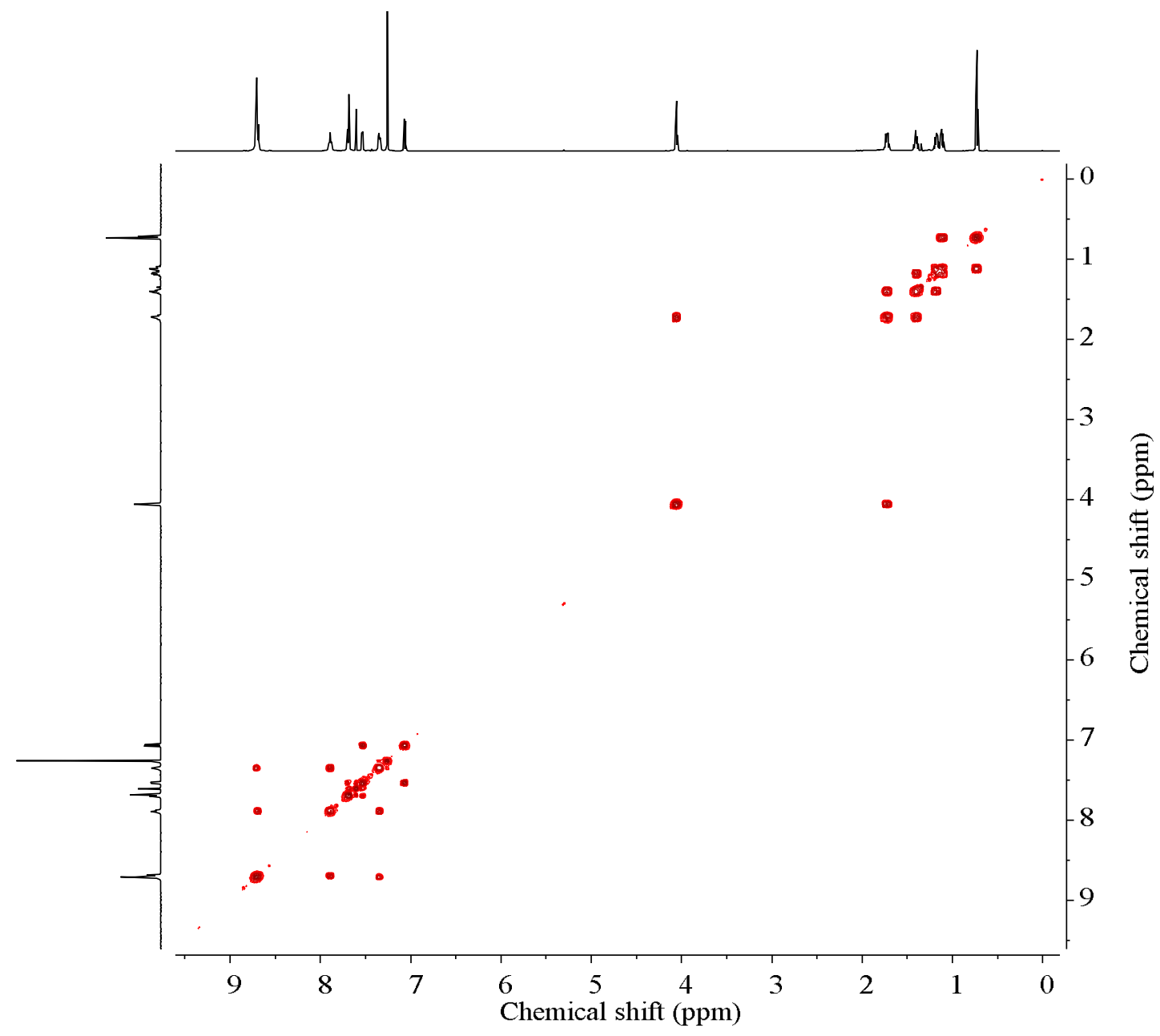

Figure S111. 2D COSY NMR (600 MHz, $\left.\mathrm{CDCl}_{3}\right)$ spectrum of compound 20.

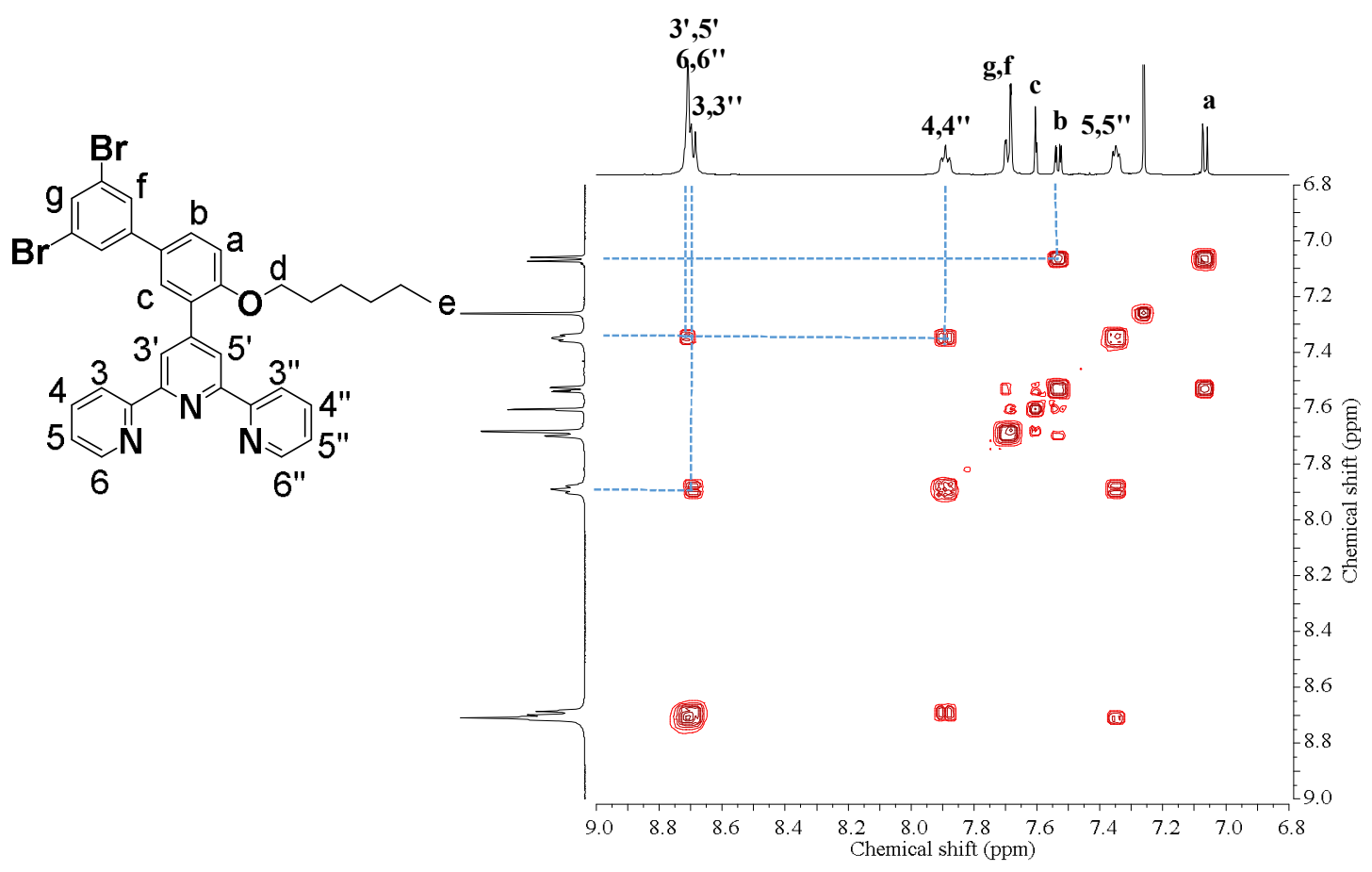

Figure S112. 2D COSY NMR (600 $\left.\mathrm{MHz}, \mathrm{CDCl}_{3}\right)$ spectrum of compound 20 (aromatic region). 


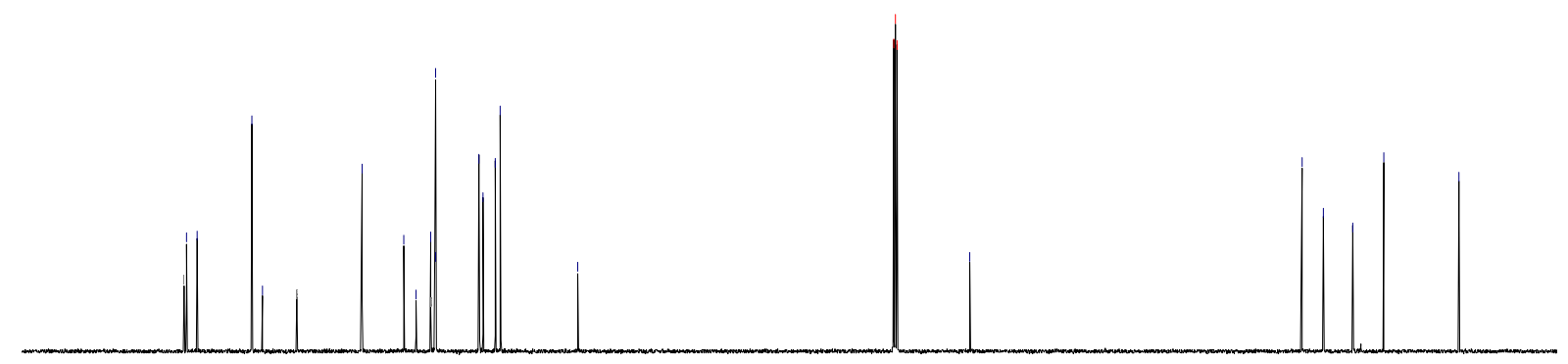

$\begin{array}{lllllllllllllll}170 & 160 & 150 & 140 & 130 & 120 & 110 & \begin{array}{c}100 \\ \text { Chemical shift }(\mathrm{ppm})\end{array}\end{array}$

Figure S113. ${ }^{13} \mathrm{C}$ NMR $\left(150 \mathrm{MHz}, \mathrm{CDCl}_{3}\right)$ spectrum of compound 20.

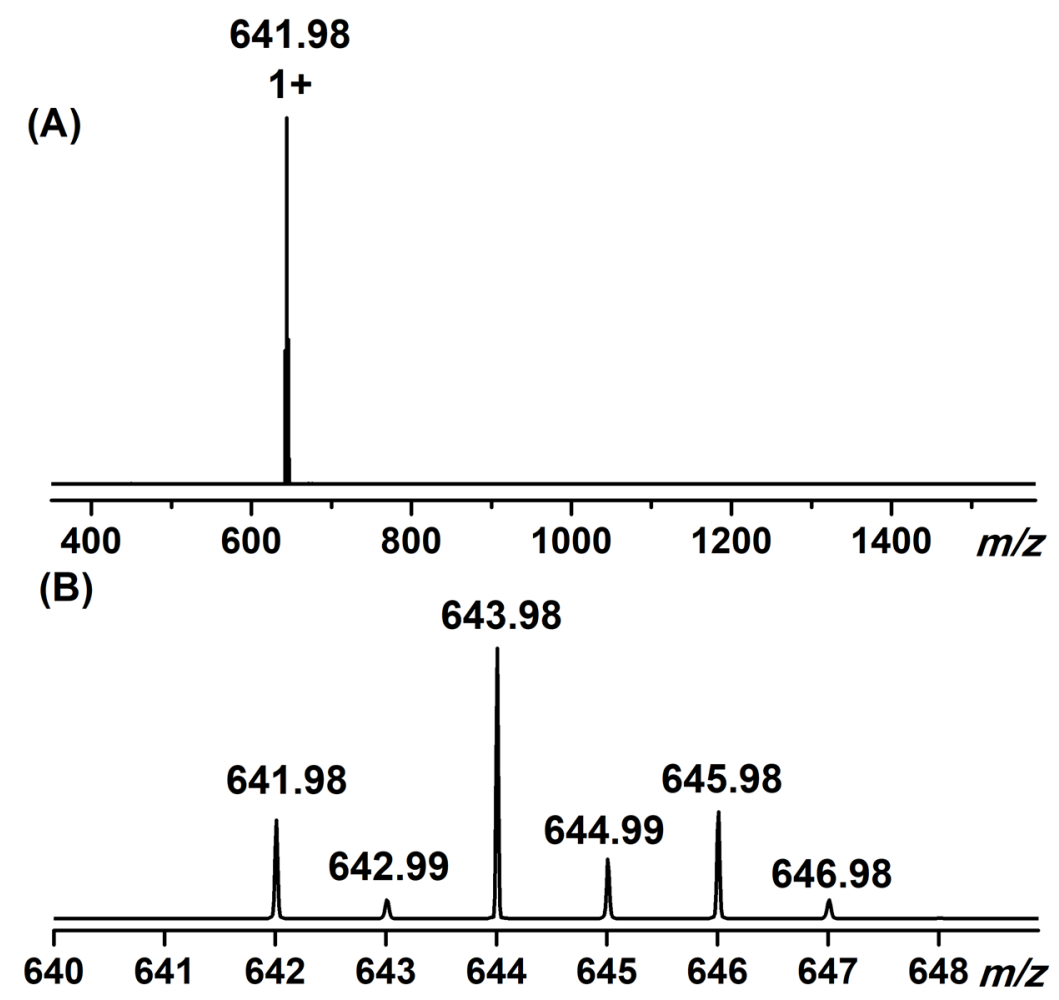

Figure S114. (A) ESI-MS spectrum of compound 20 in $\mathrm{CHCl}_{3} / \mathrm{CH}_{3} \mathrm{OH}(1 / 3)$ and (B) isotope pattern. 


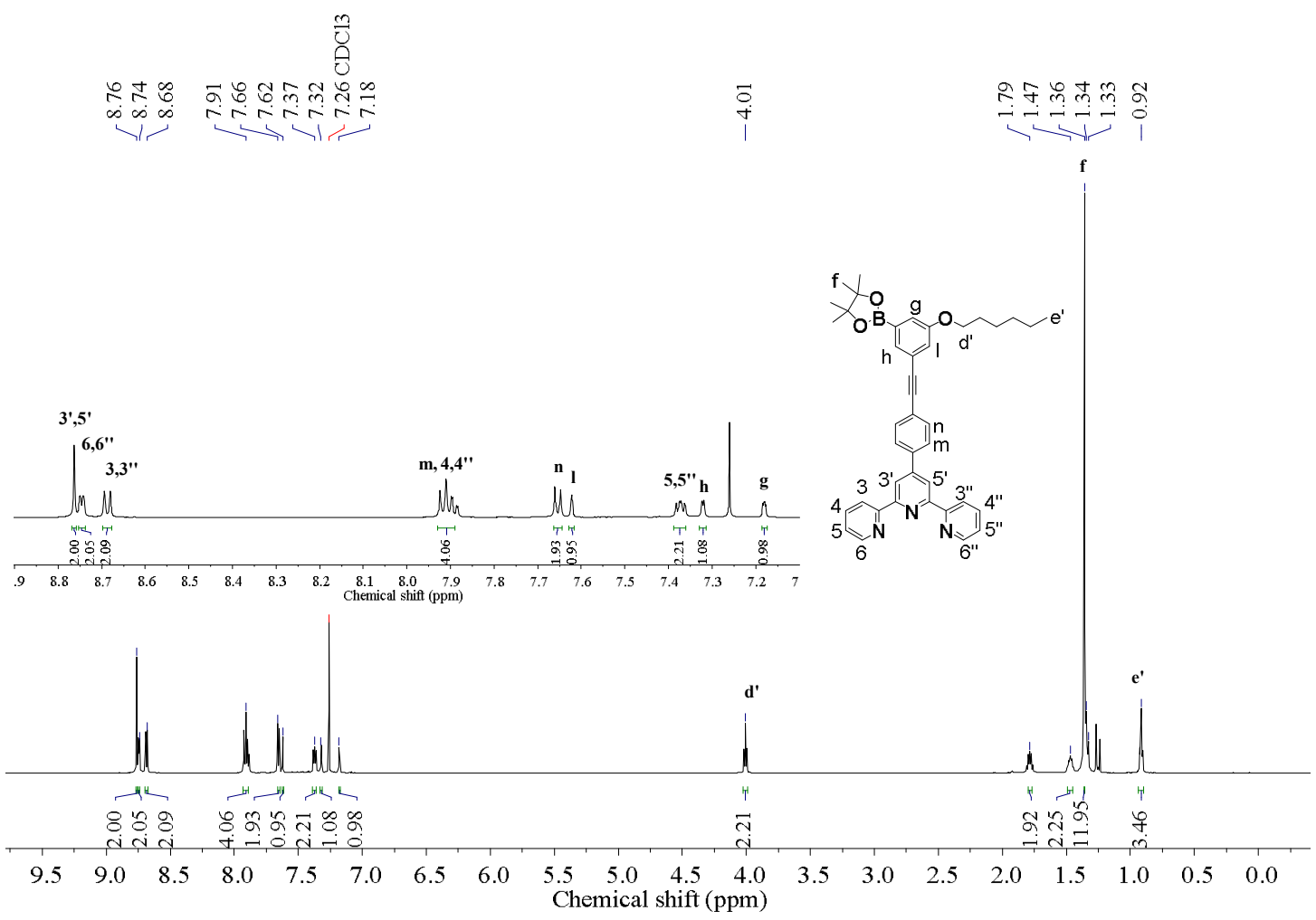

Figure S115. ${ }^{1} \mathrm{H}$ NMR $\left(600 \mathrm{MHz}, \mathrm{CDCl}_{3}\right)$ spectrum of compound 21.

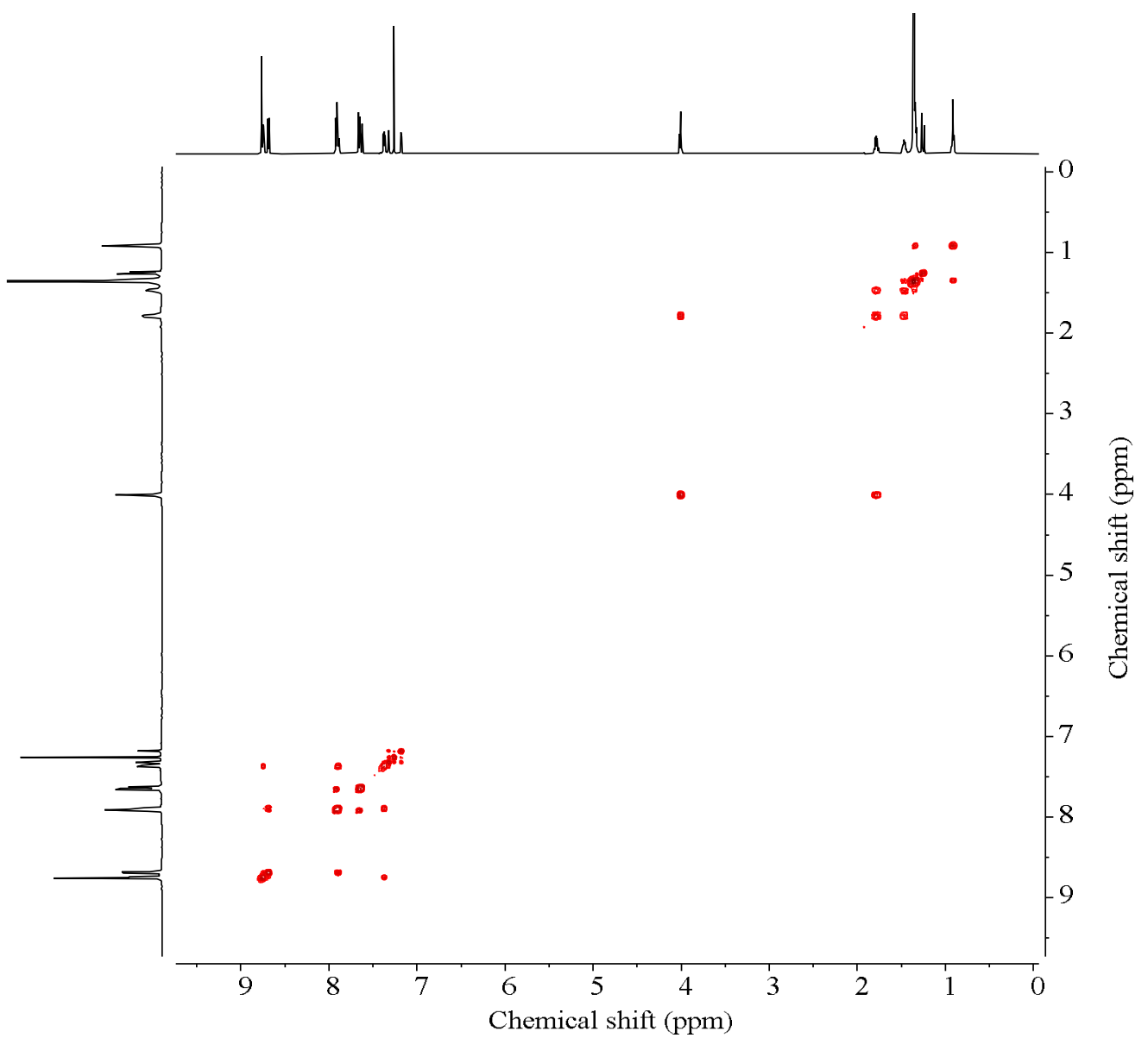

Figure S116. 2D COSY NMR (600 MHz, $\left.\mathrm{CDCl}_{3}\right)$ spectrum of compound 21. 


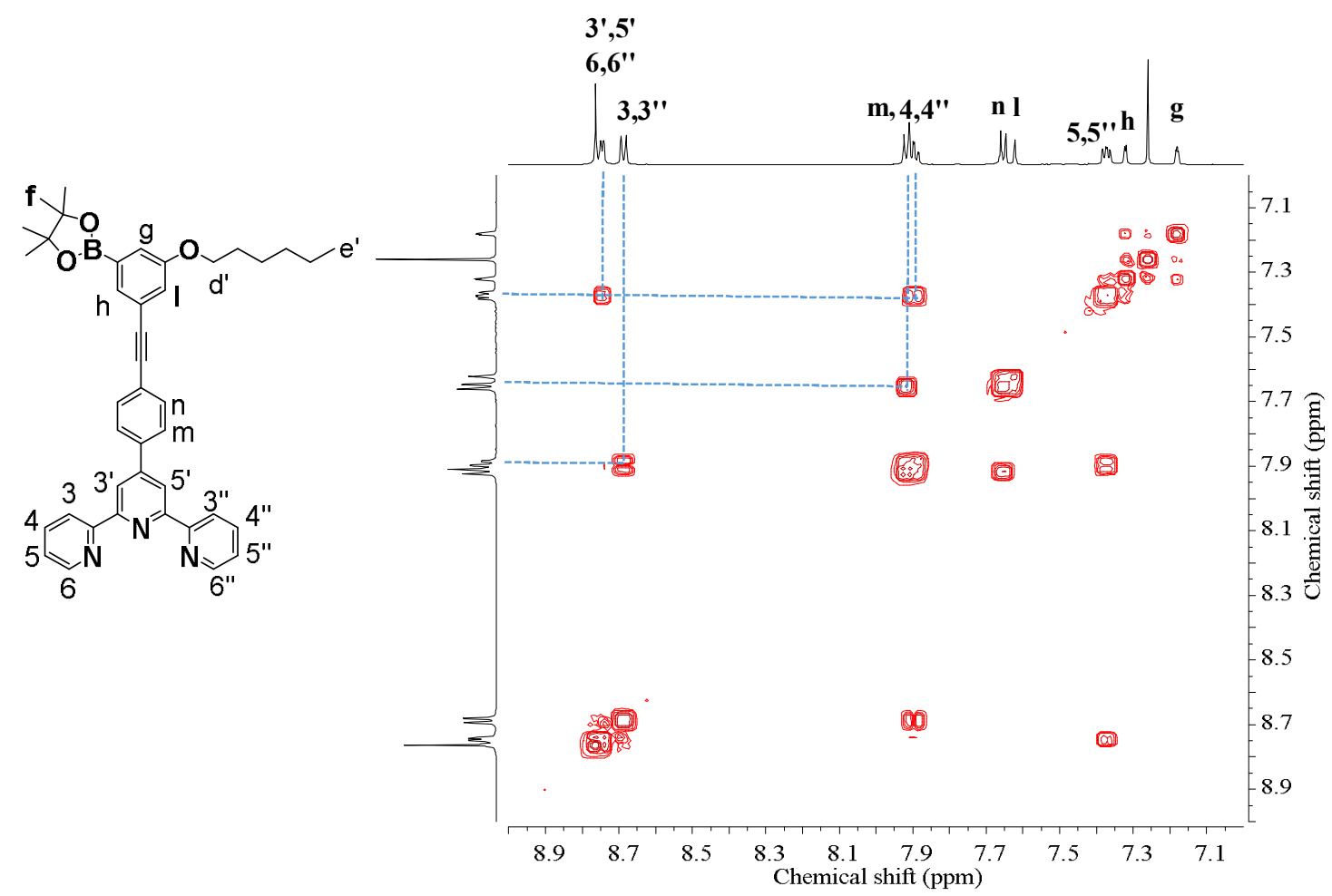

Figure S117. 2D COSY NMR (600 $\left.\mathrm{MHz}, \mathrm{CDCl}_{3}\right)$ spectrum of compound 21 (aromatic region).
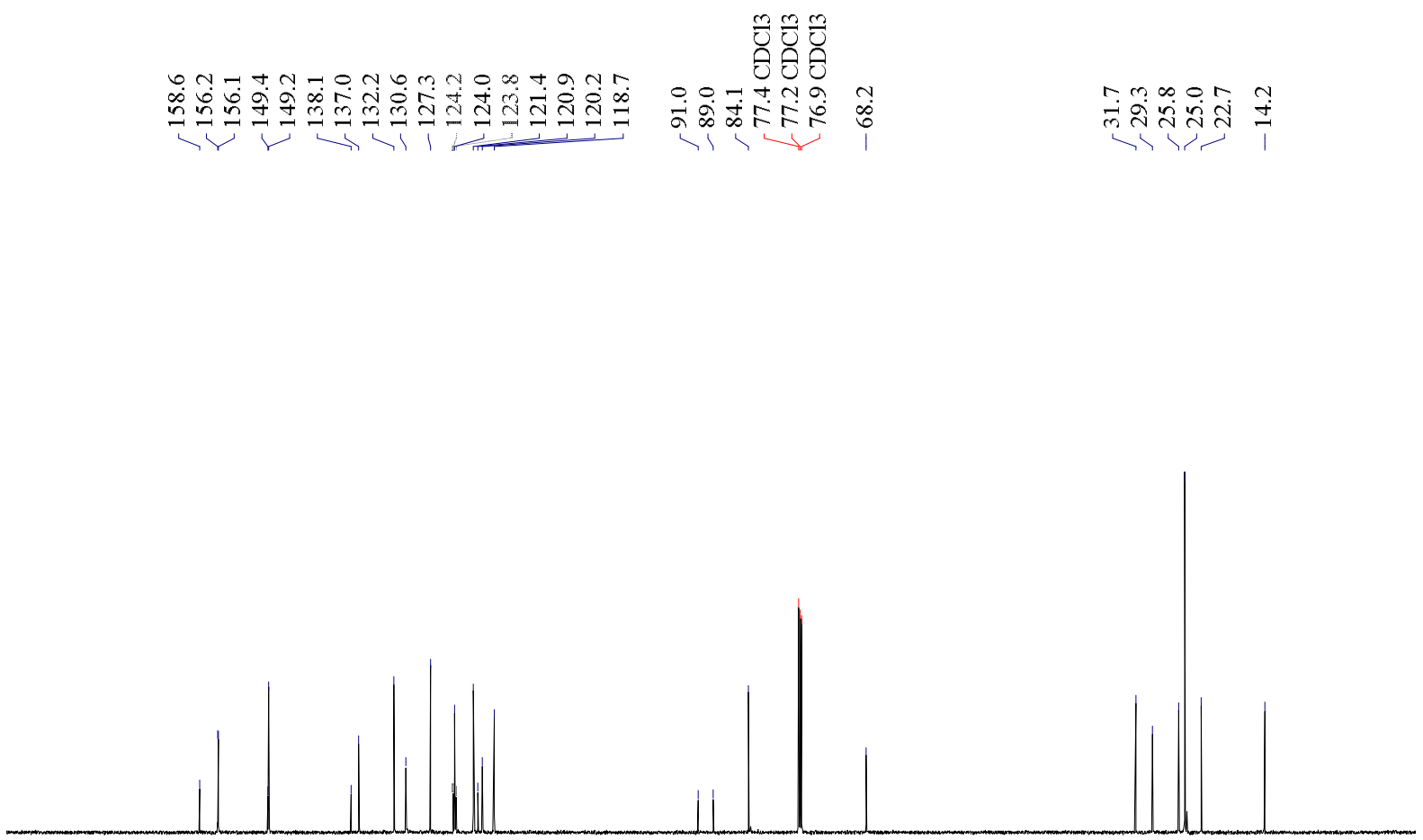

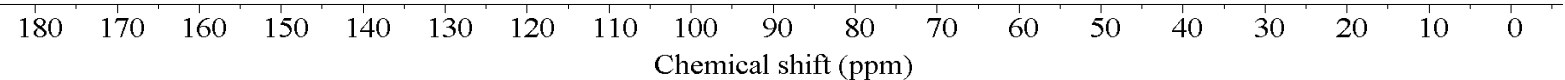

Figure S118. ${ }^{13} \mathrm{C} \mathrm{NMR}\left(150 \mathrm{MHz}, \mathrm{CDCl}_{3}\right)$ spectrum of compound 21. 

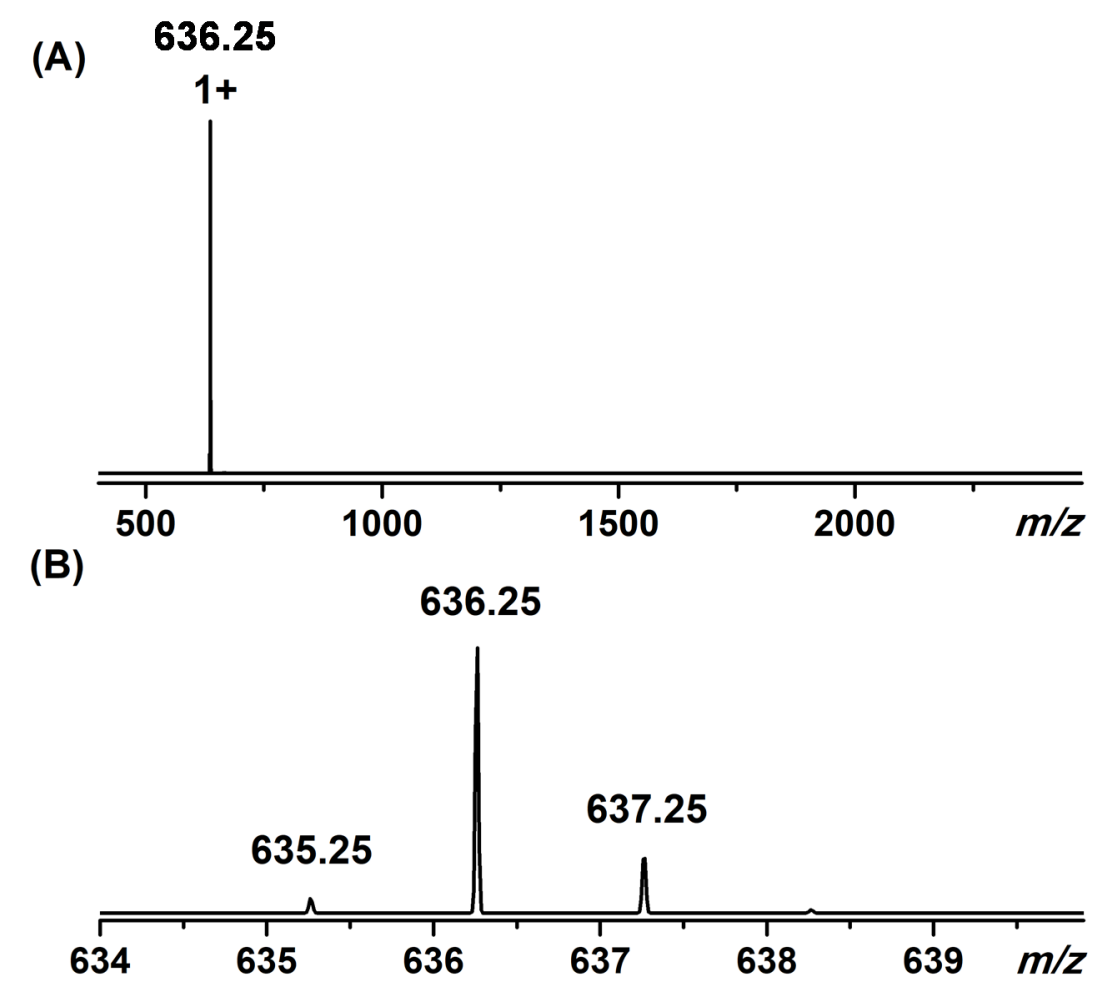

Figure S119. (A) ESI-MS spectrum of compound 21 in $\mathrm{CHCl}_{3} / \mathrm{CH}_{3} \mathrm{OH}(1 / 3)$ and (B) isotope pattern.
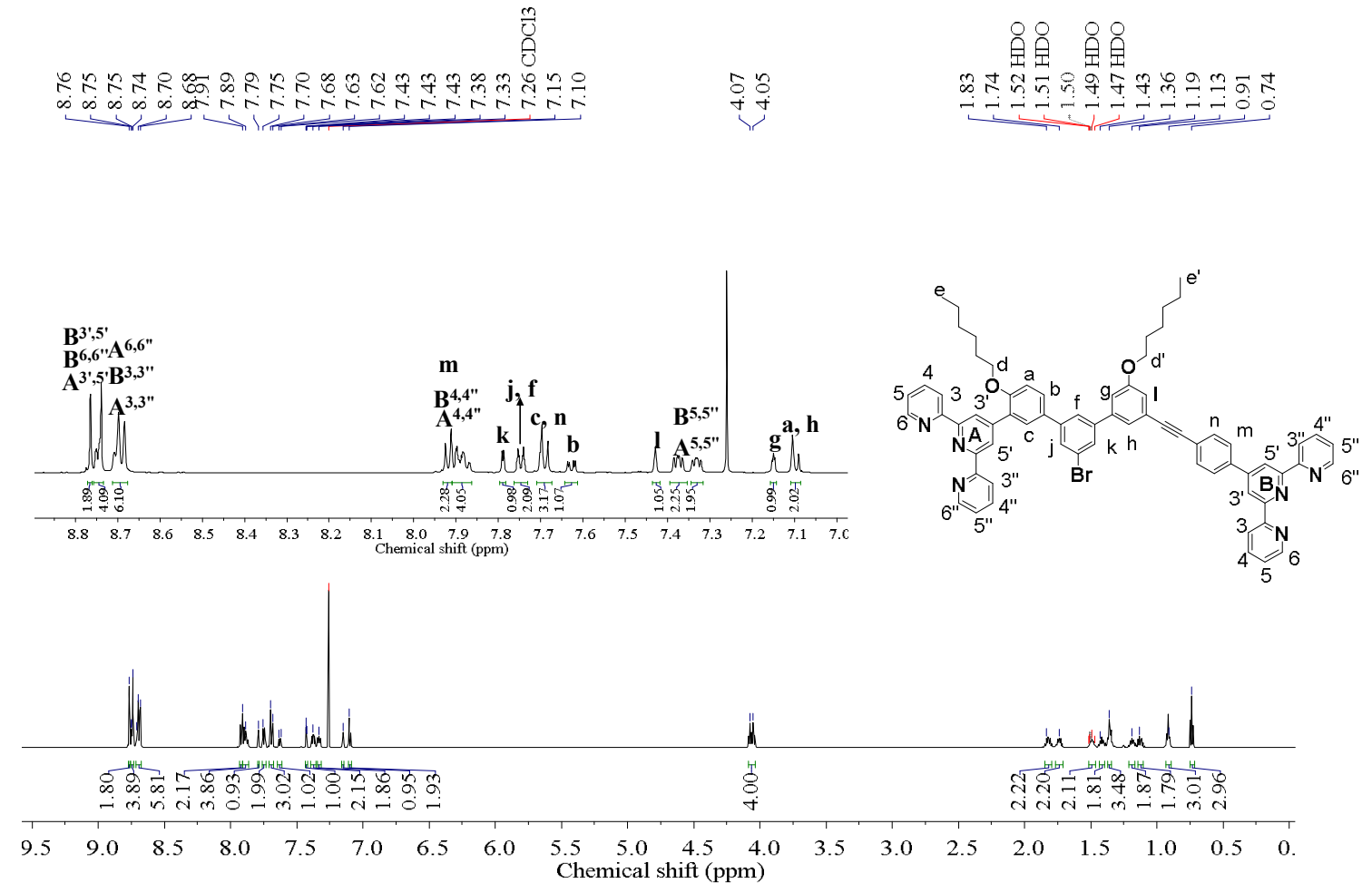

Figure S120. ${ }^{1} \mathrm{H}$ NMR $\left(600 \mathrm{MHz}, \mathrm{CDCl}_{3}\right)$ spectrum of compound 22. 


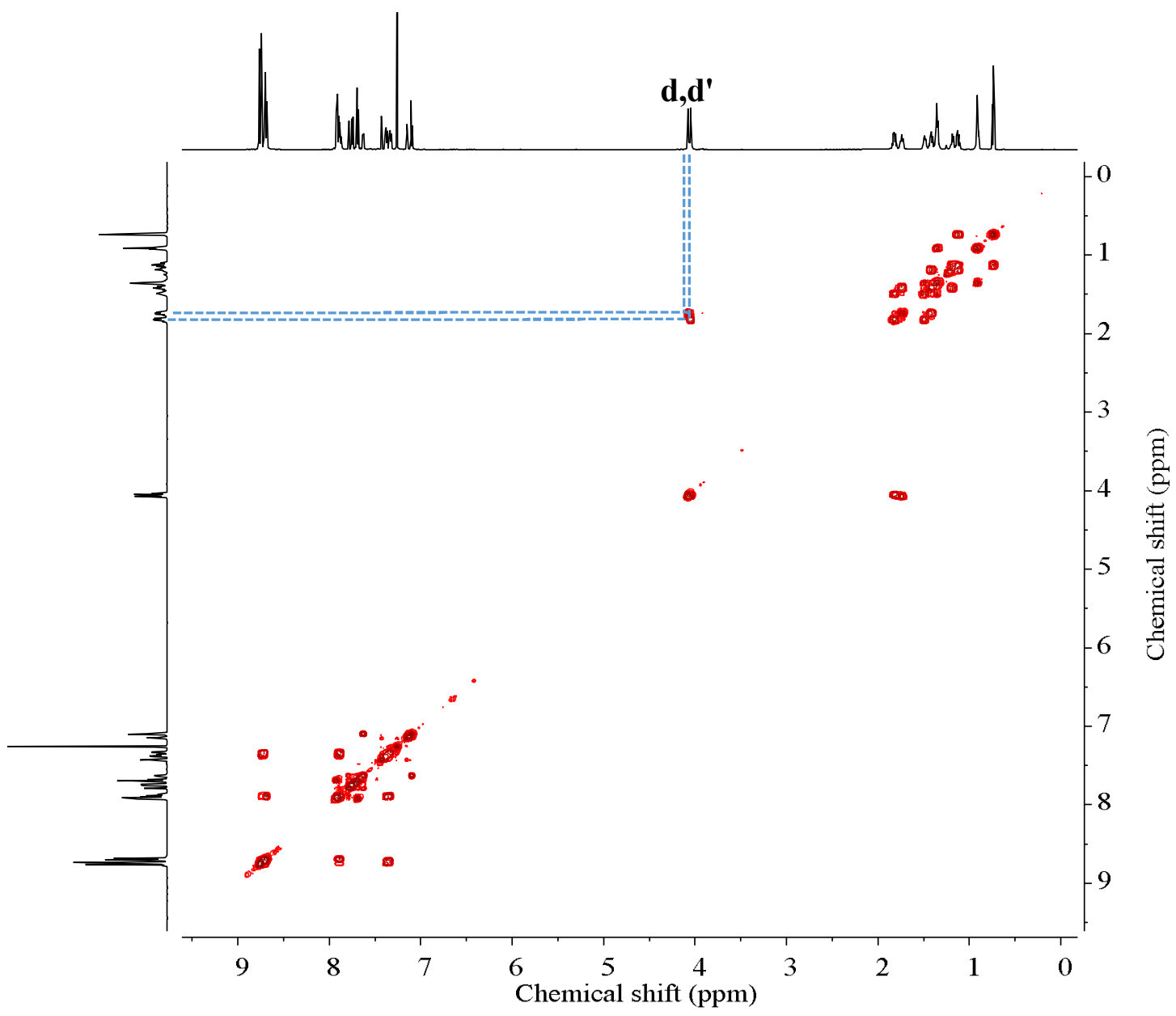

Figure S121. 2D COSY NMR (600 MHz, $\mathrm{CDCl}_{3}$ ) spectrum of compound 22.

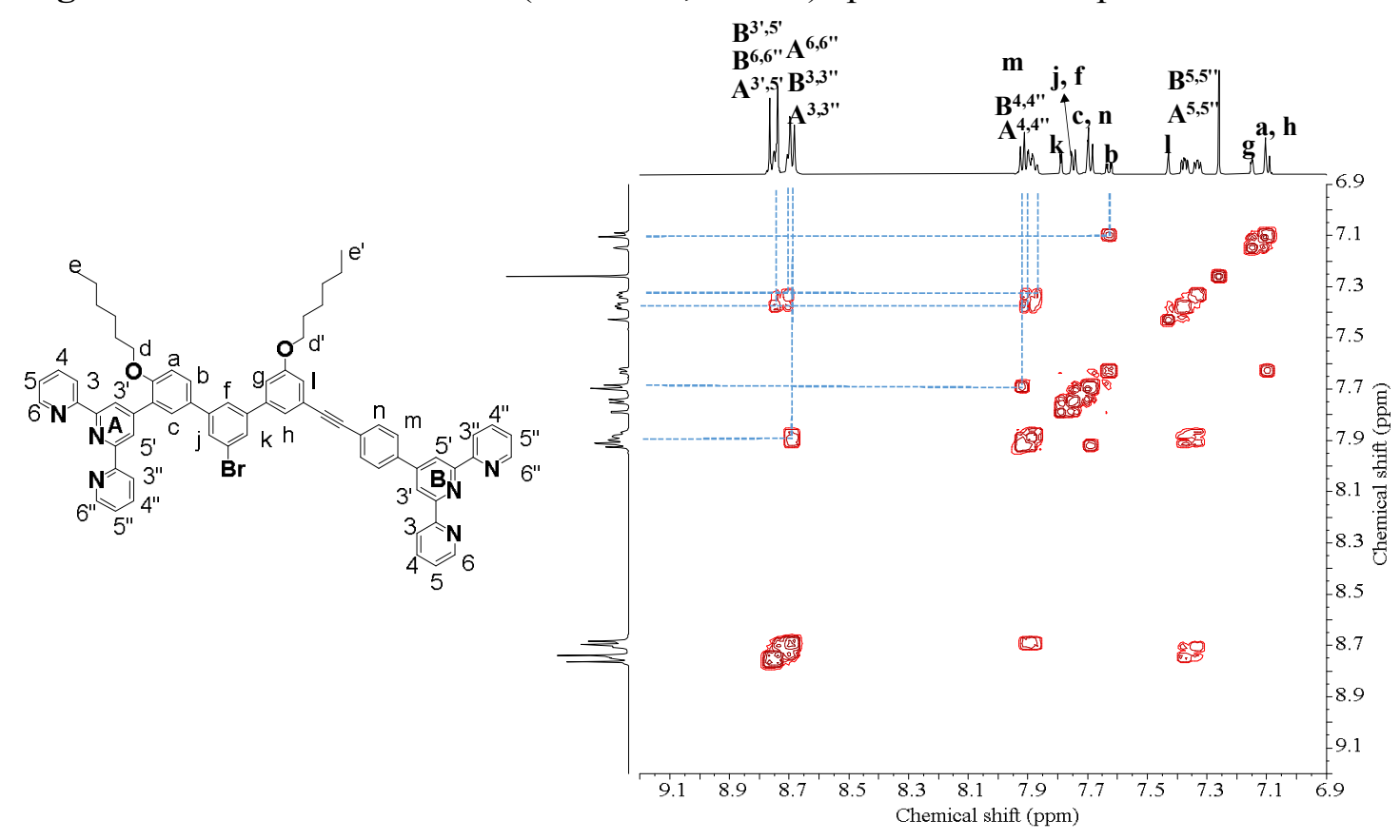

Figure S122. 2D COSY NMR (600 $\left.\mathrm{MHz}, \mathrm{CDCl}_{3}\right)$ spectrum of compound 22 (aromatic region). 


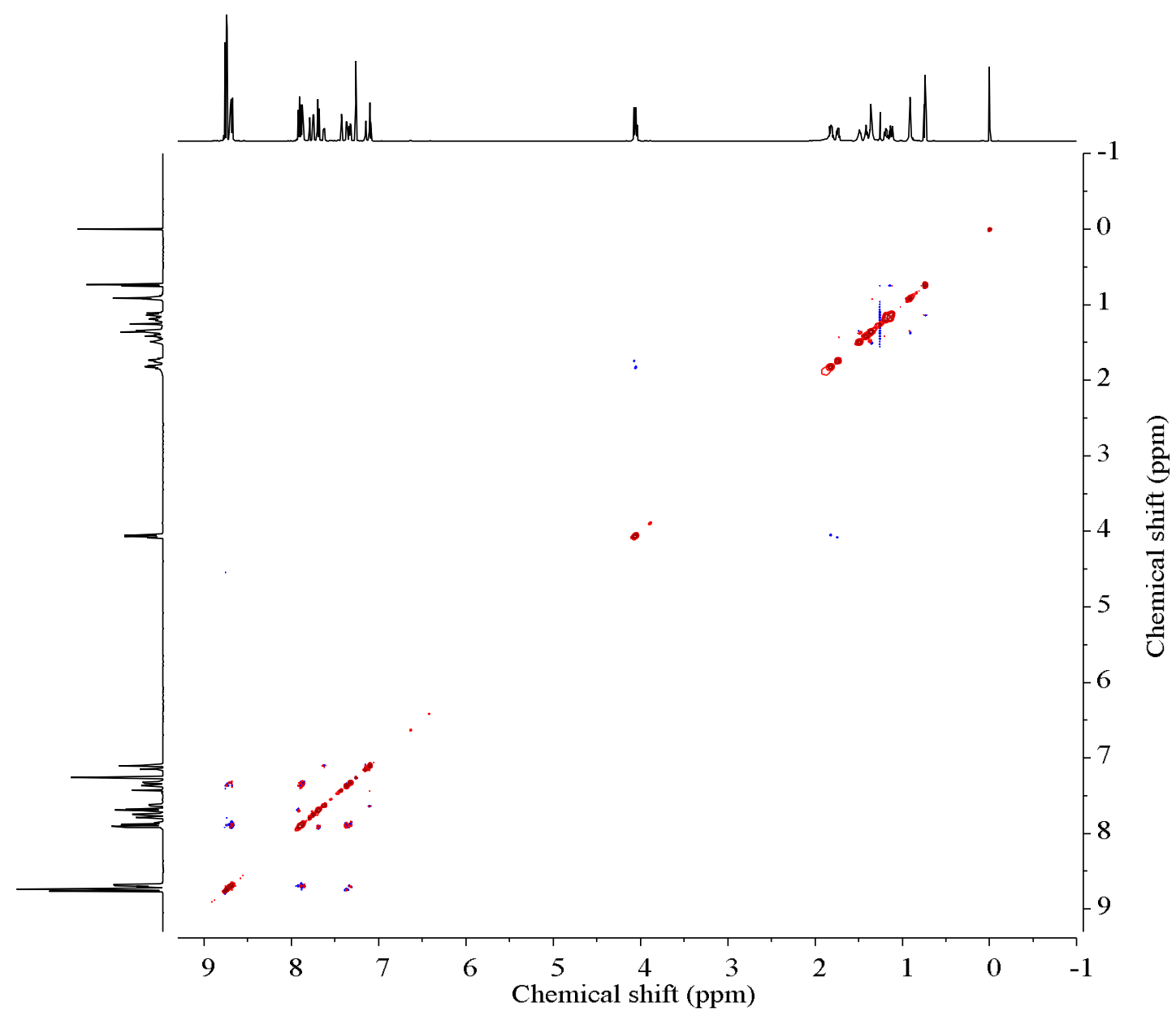

Figure S123. 2D NOESY NMR (600 MHz, $\left.\mathrm{CDCl}_{3}\right)$ spectrum of compound 22.

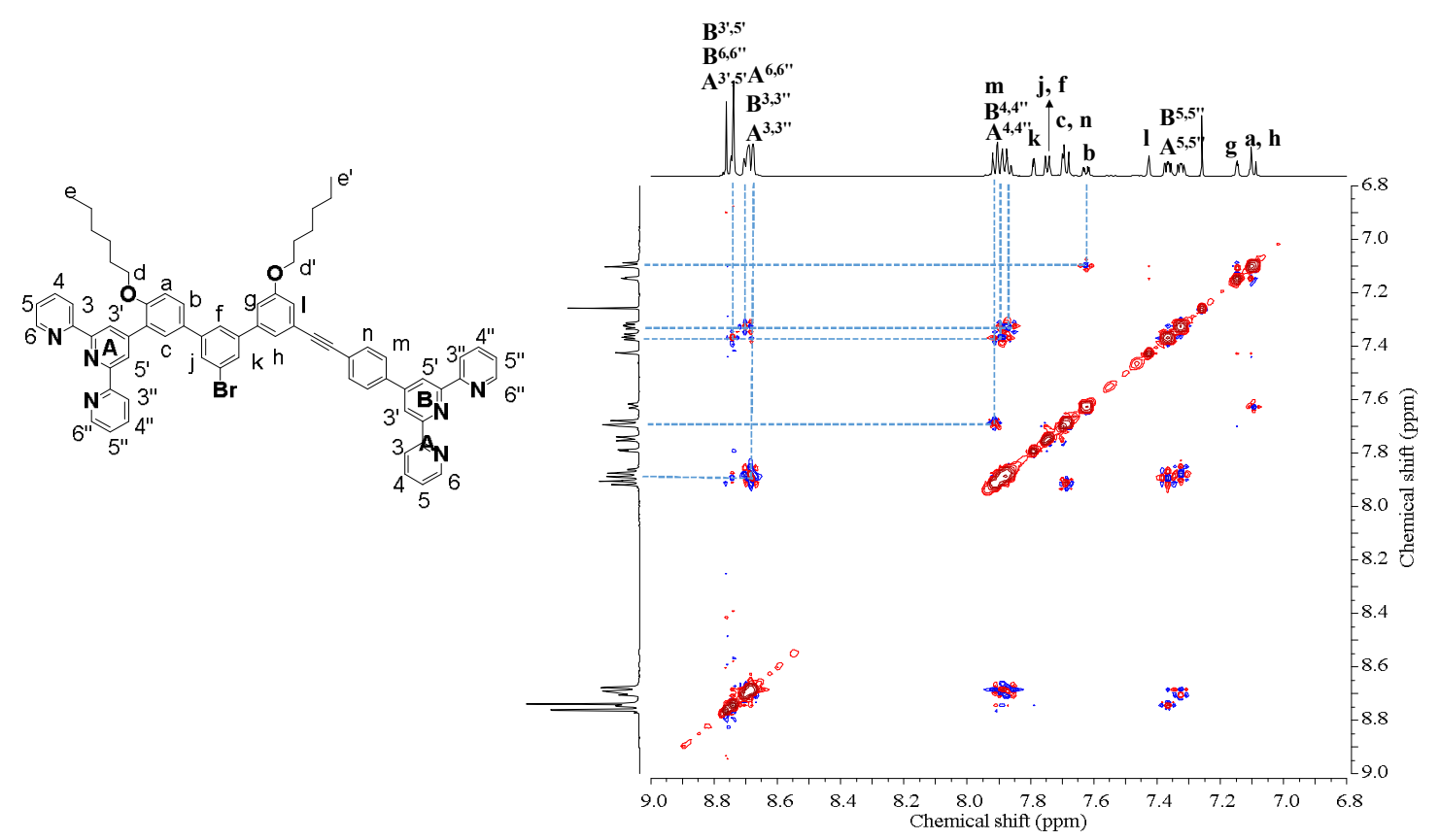

Figure S124. 2D NOESY NMR (600 $\left.\mathrm{MHz}, \mathrm{CDCl}_{3}\right)$ spectrum of compound 22 (aromatic region). 


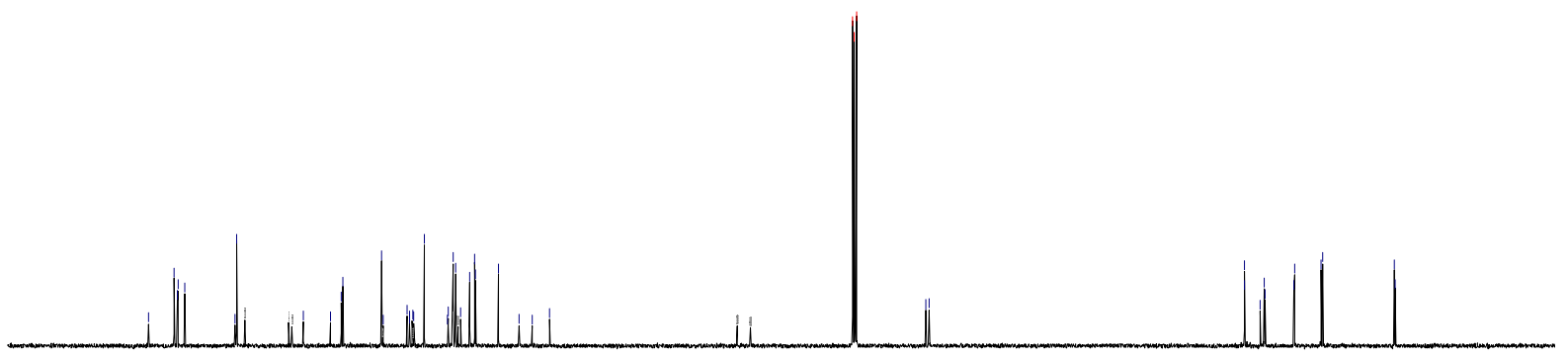

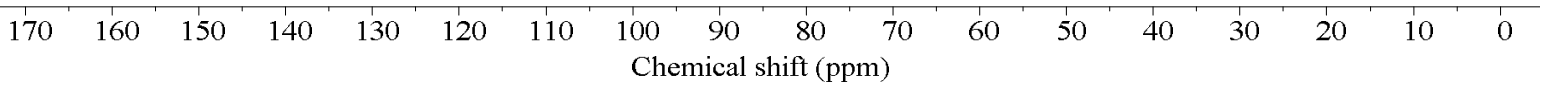

Figure S125. ${ }^{13} \mathrm{C}$ NMR (150 MHz, $\left.\mathrm{CDCl}_{3}\right)$ spectrum of compound 22.

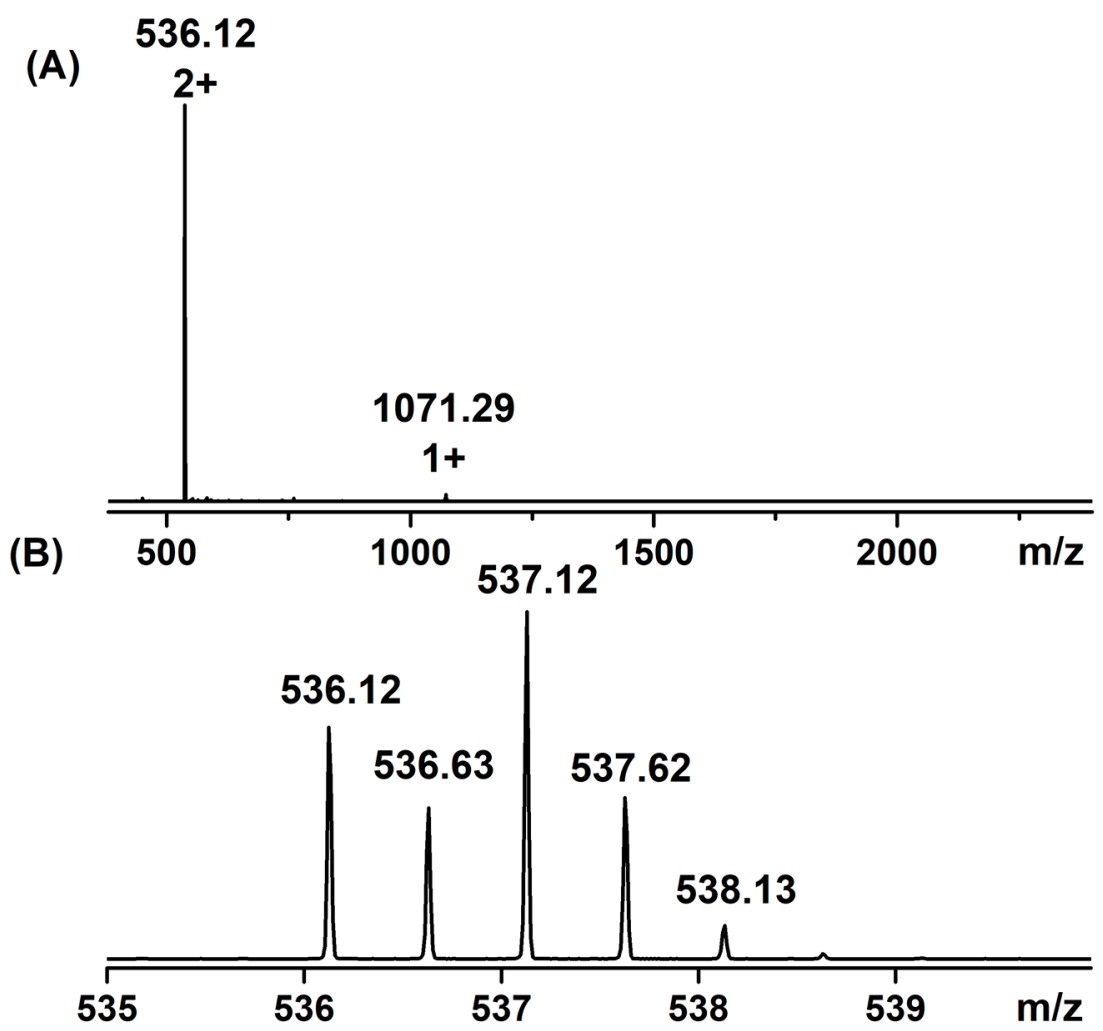

Figure S126. (A) ESI-MS spectrum of compound 22 in $\mathrm{CHCl}_{3} / \mathrm{CH}_{3} \mathrm{OH}(1 / 3)$ and (B) isotope pattern. 


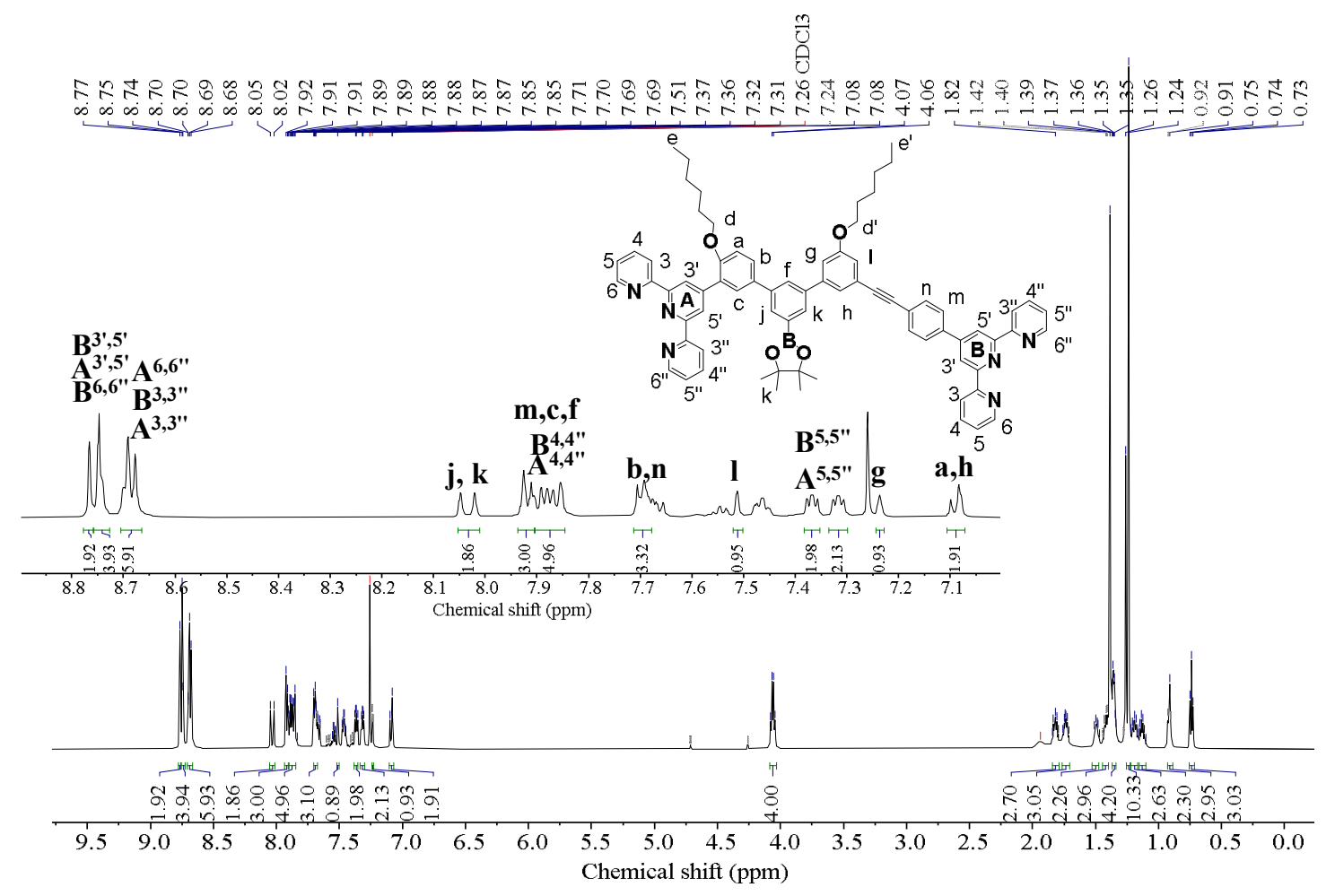

Figure S127. ${ }^{1} \mathrm{H} \mathrm{NMR}\left(600 \mathrm{MHz}, \mathrm{CDCl}_{3}\right)$ spectrum of compound 23.

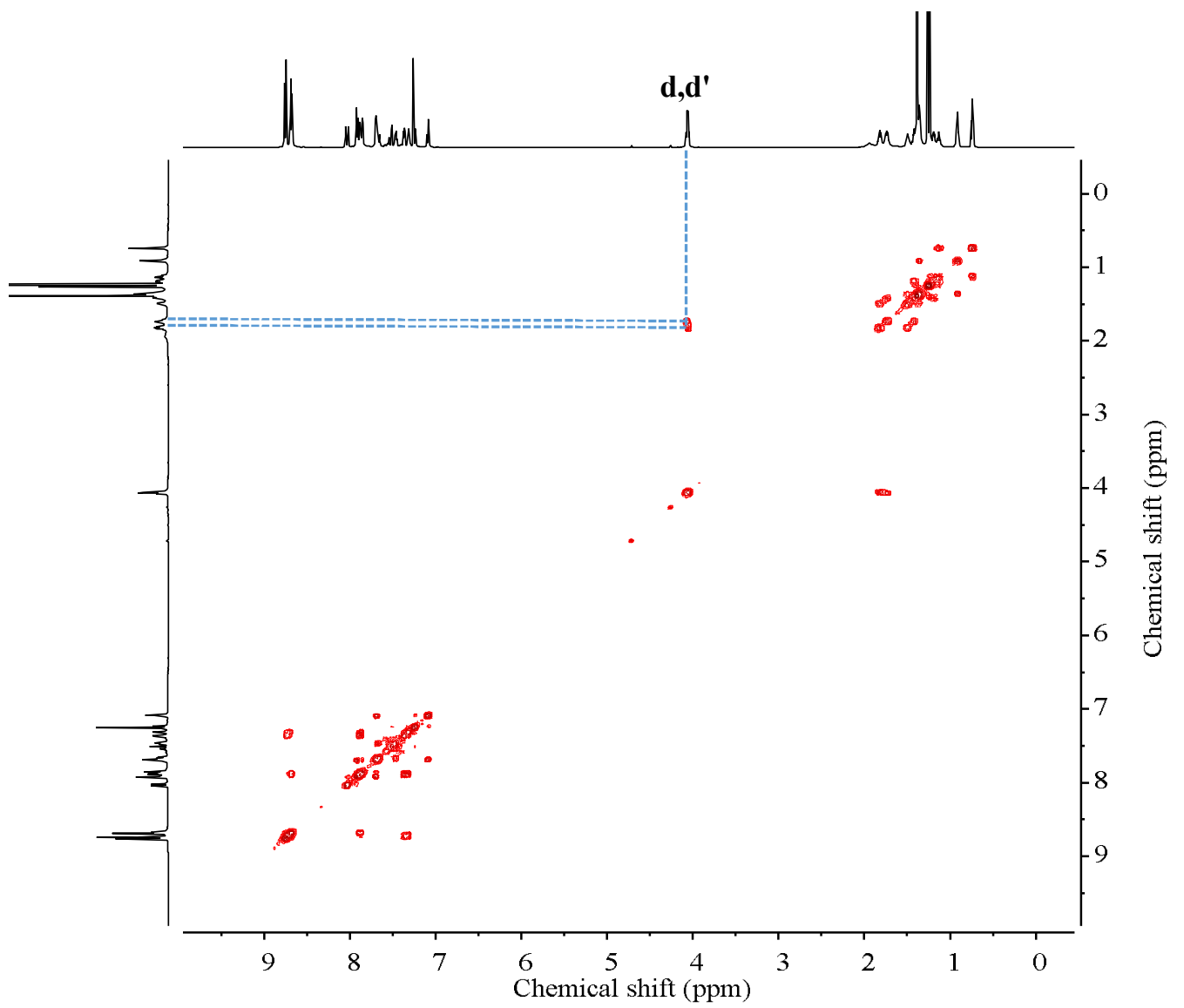

Figure S128. 2D COSY NMR (600 MHz, $\left.\mathrm{CDCl}_{3}\right)$ spectrum of compound 23. 


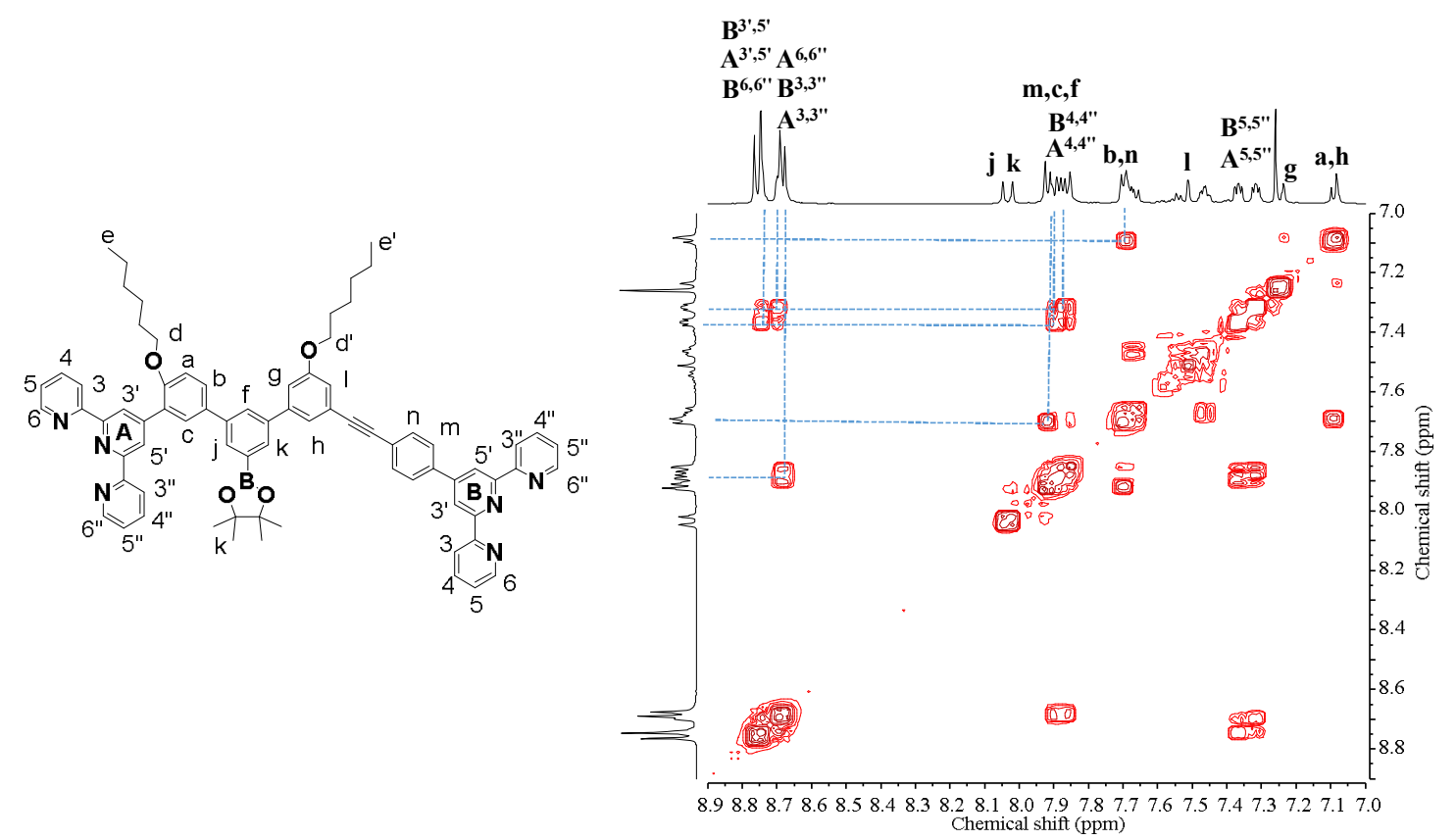

Figure S129. 2D COSY NMR (600 $\left.\mathrm{MHz}, \mathrm{CDCl}_{3}\right)$ spectrum of compound 23 (aromatic region).

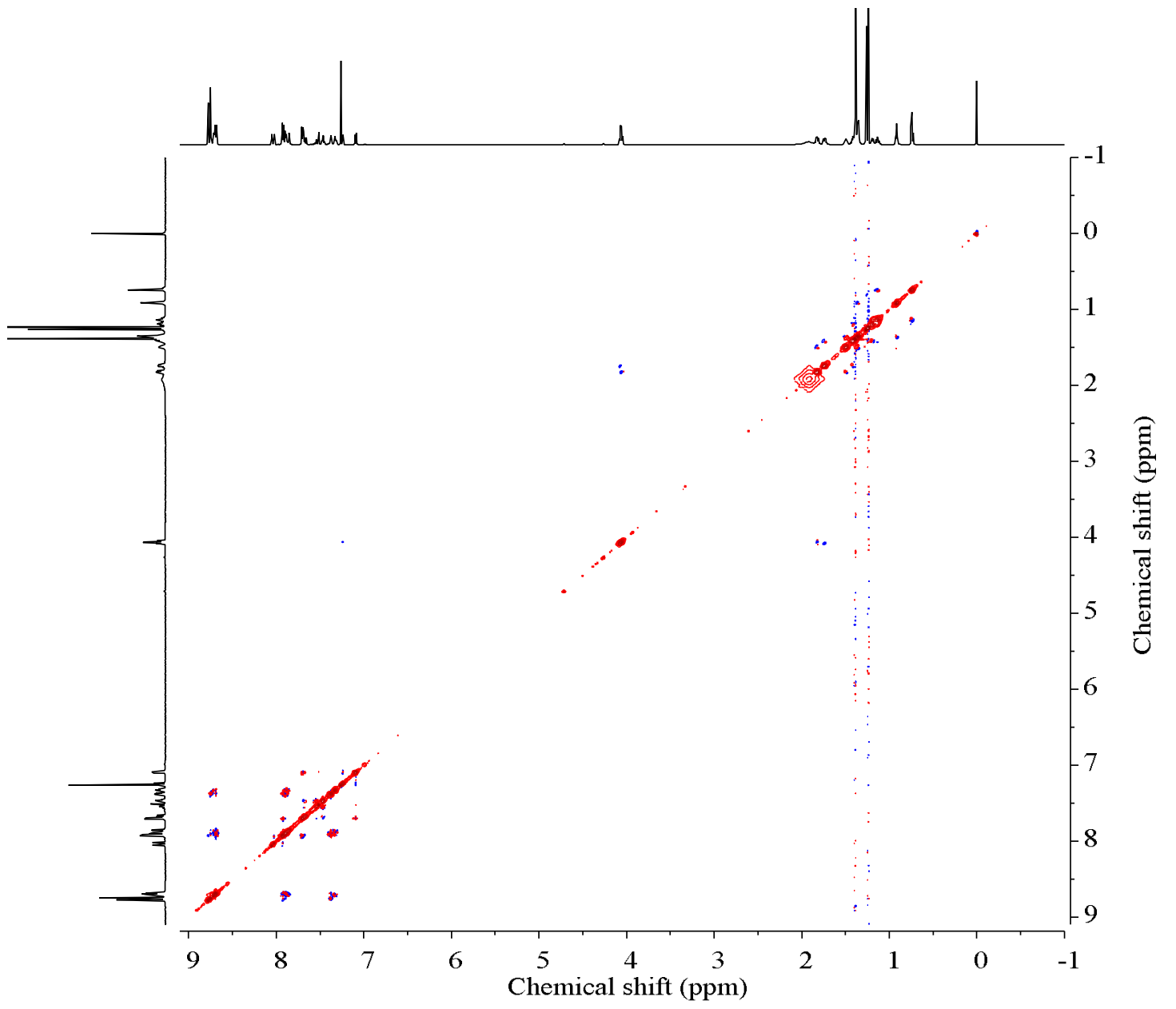

Figure S130. 2D NOESY NMR (600 MHz, $\left.\mathrm{CDCl}_{3}\right)$ spectrum of compound 23. 


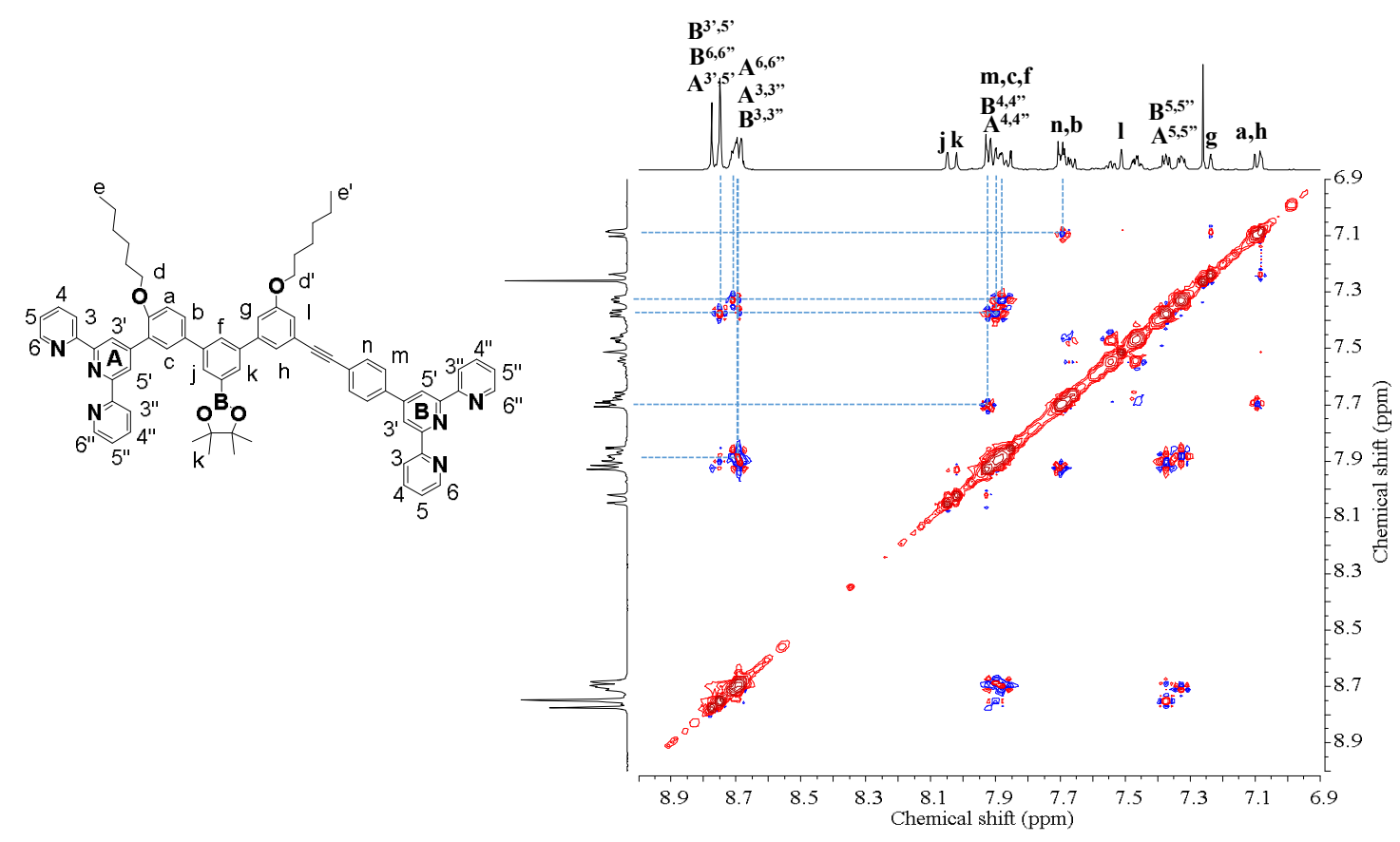

Figure S131. 2D NOESY NMR (600 $\left.\mathrm{MHz}, \mathrm{CDCl}_{3}\right)$ spectrum of compound 23 (aromatic region).

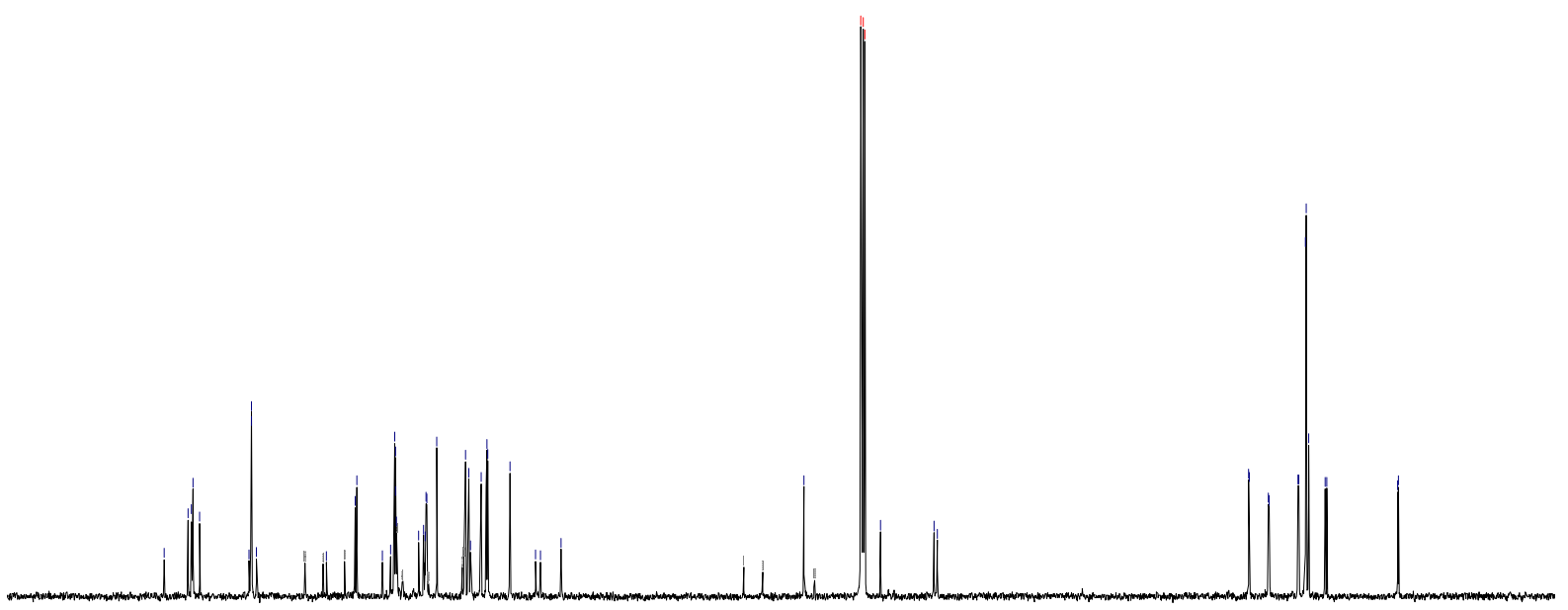

$\begin{array}{llllllllllllllllll}170 & 160 & 150 & 140 & 130 & 120 & 110 & 100 & 90 & 80 & 70 & 60 & 50 & 40 & 30 & 20 & 10 & 0 \\ \text { Chemical shift (ppm) }\end{array}$

Figure S132. ${ }^{13} \mathrm{C} \mathrm{NMR}\left(150 \mathrm{MHz}, \mathrm{CDCl}_{3}\right)$ spectrum of compound 23. 


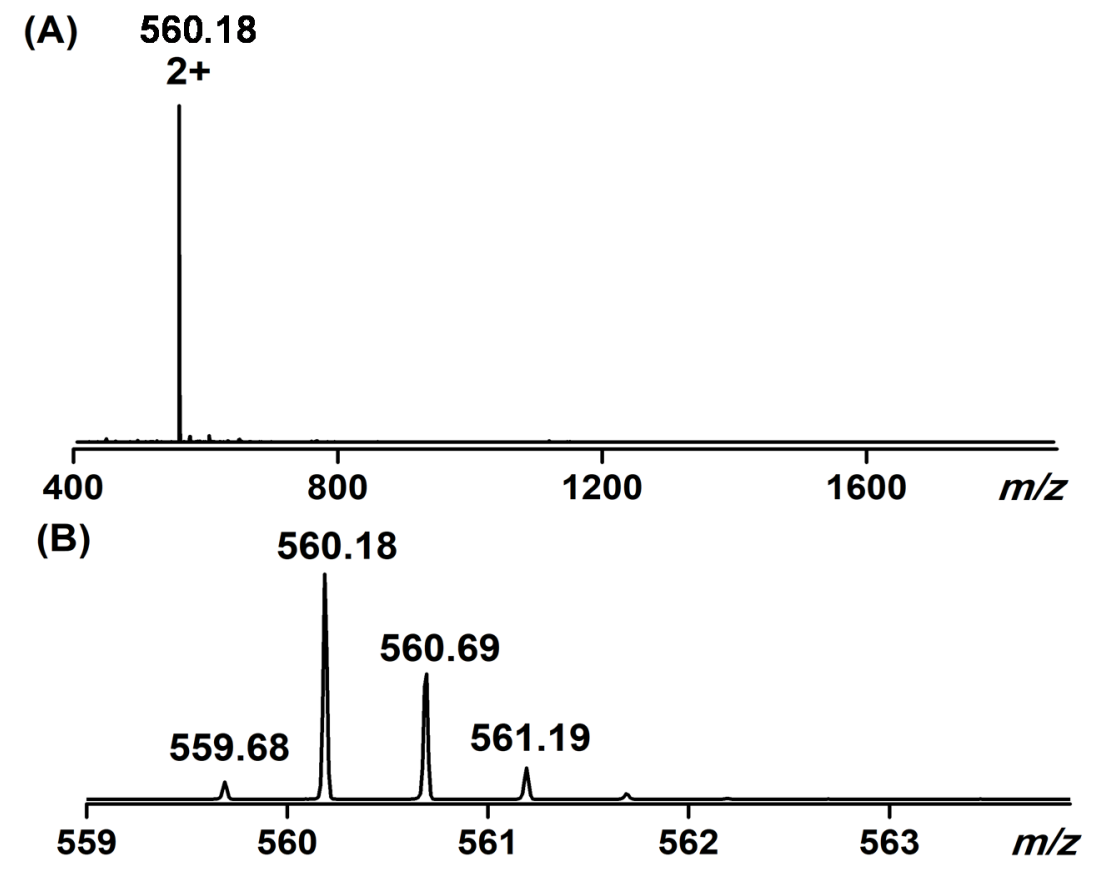

Figure S133. (A) ESI-MS spectrum of compound 23 in $\mathrm{CHCl}_{3} / \mathrm{CH}_{3} \mathrm{OH}(1 / 3)$ and isotope pattern (B).

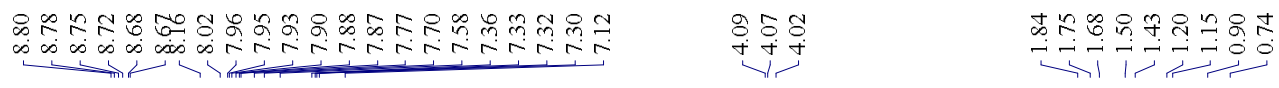

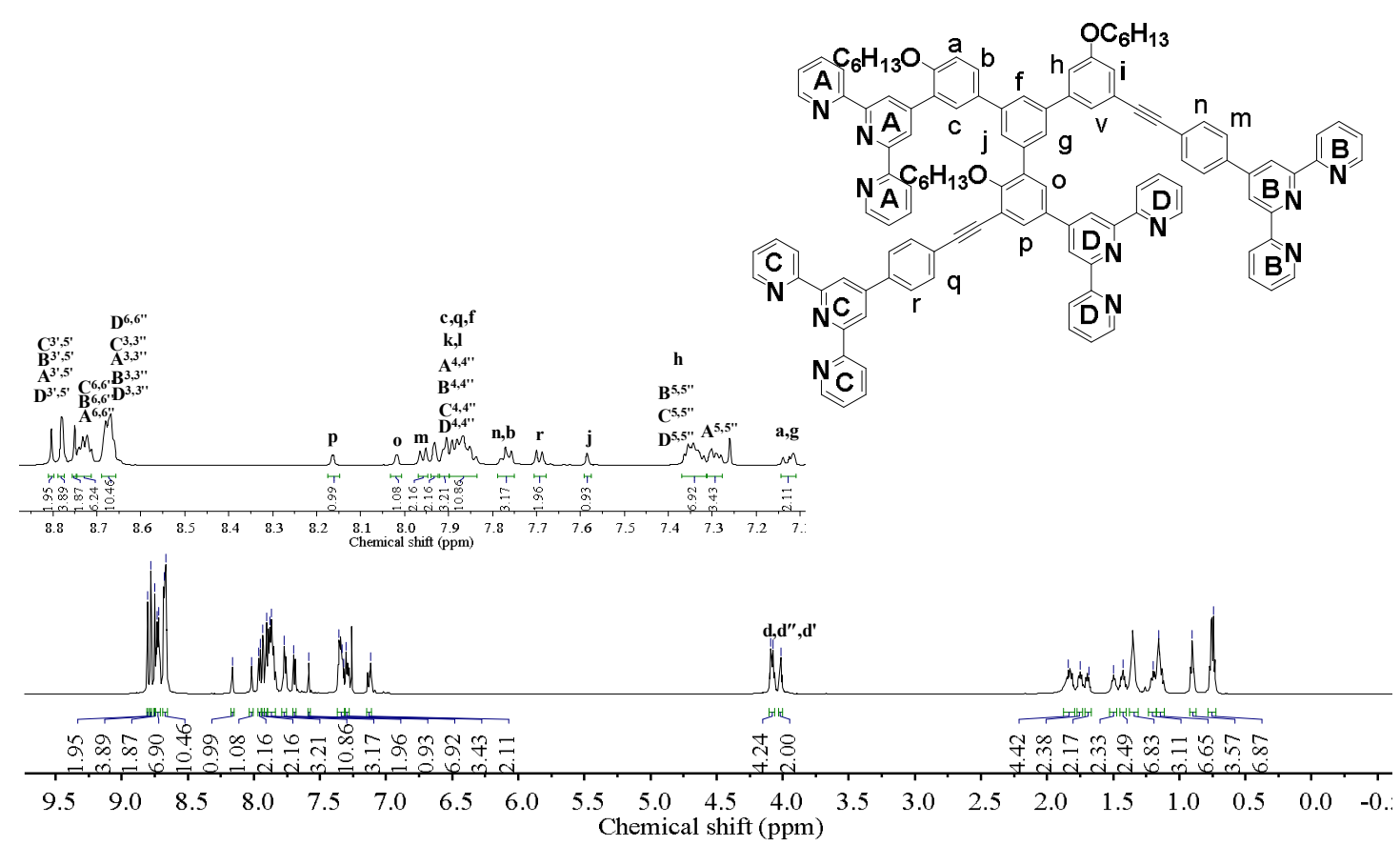

Figure S134. ${ }^{1} \mathrm{H}$ NMR (600 MHz, $\left.\mathrm{CDCl}_{3}\right)$ spectrum of ligand $\mathbf{L B}$. 


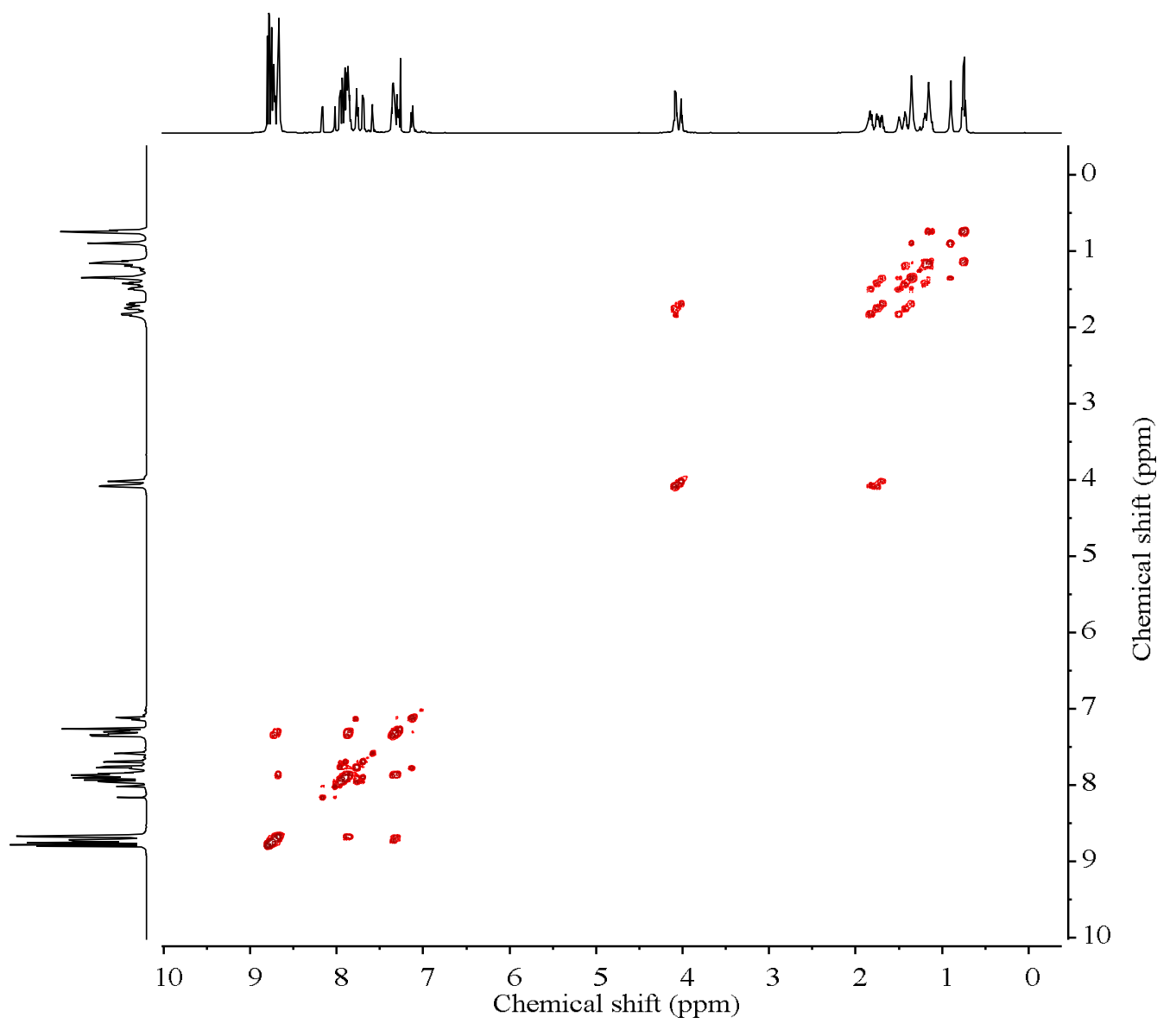

Figure S135. 2D COSY NMR (600 MHz, $\left.\mathrm{CDCl}_{3}\right)$ spectrum of ligand $\mathbf{L B}$.

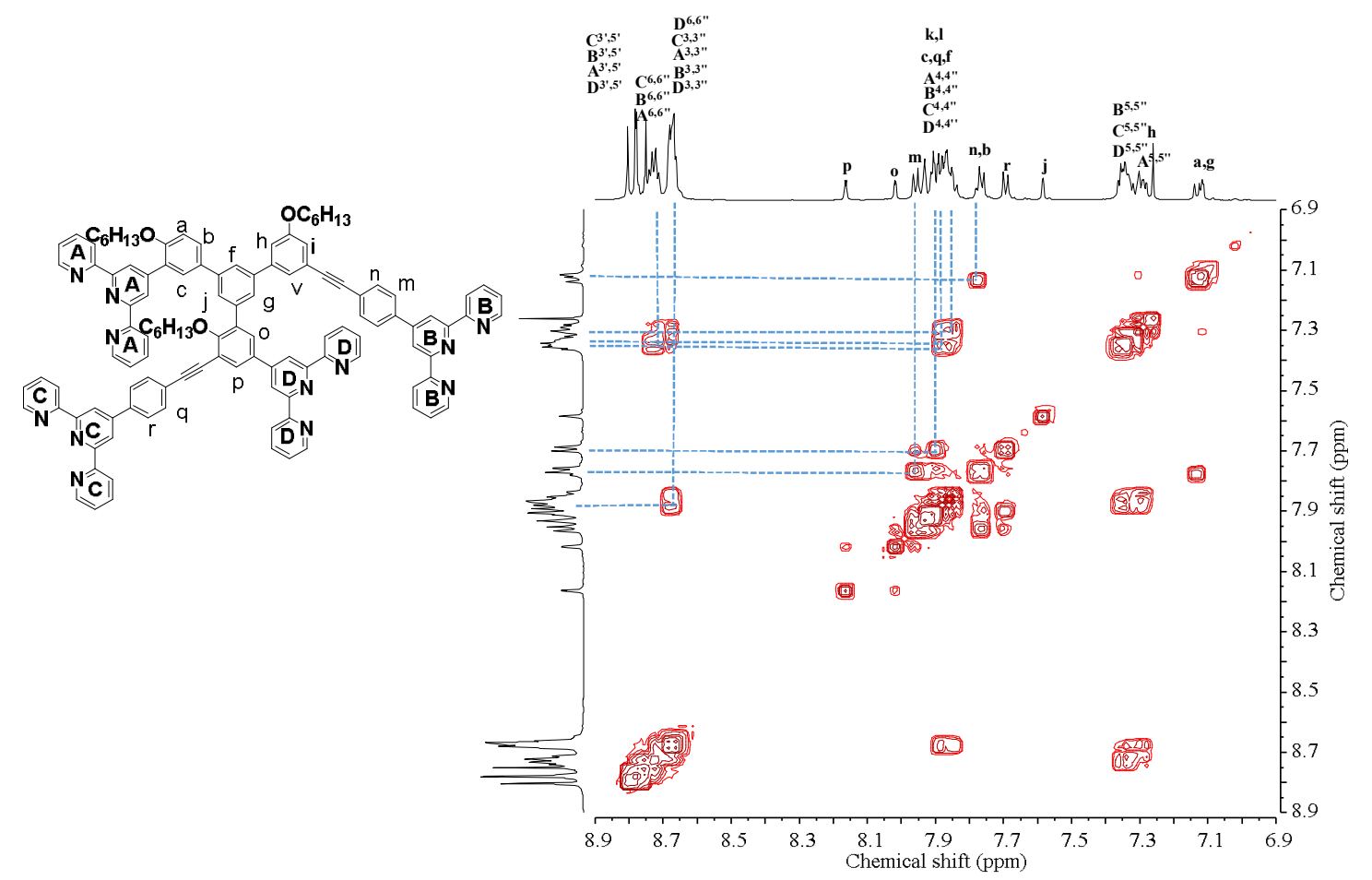

Figure S136. 2D COSY NMR (600 MHz, $\left.\mathrm{CDCl}_{3}\right)$ spectrum of ligand $\mathbf{L B}$ (aromatic region). 


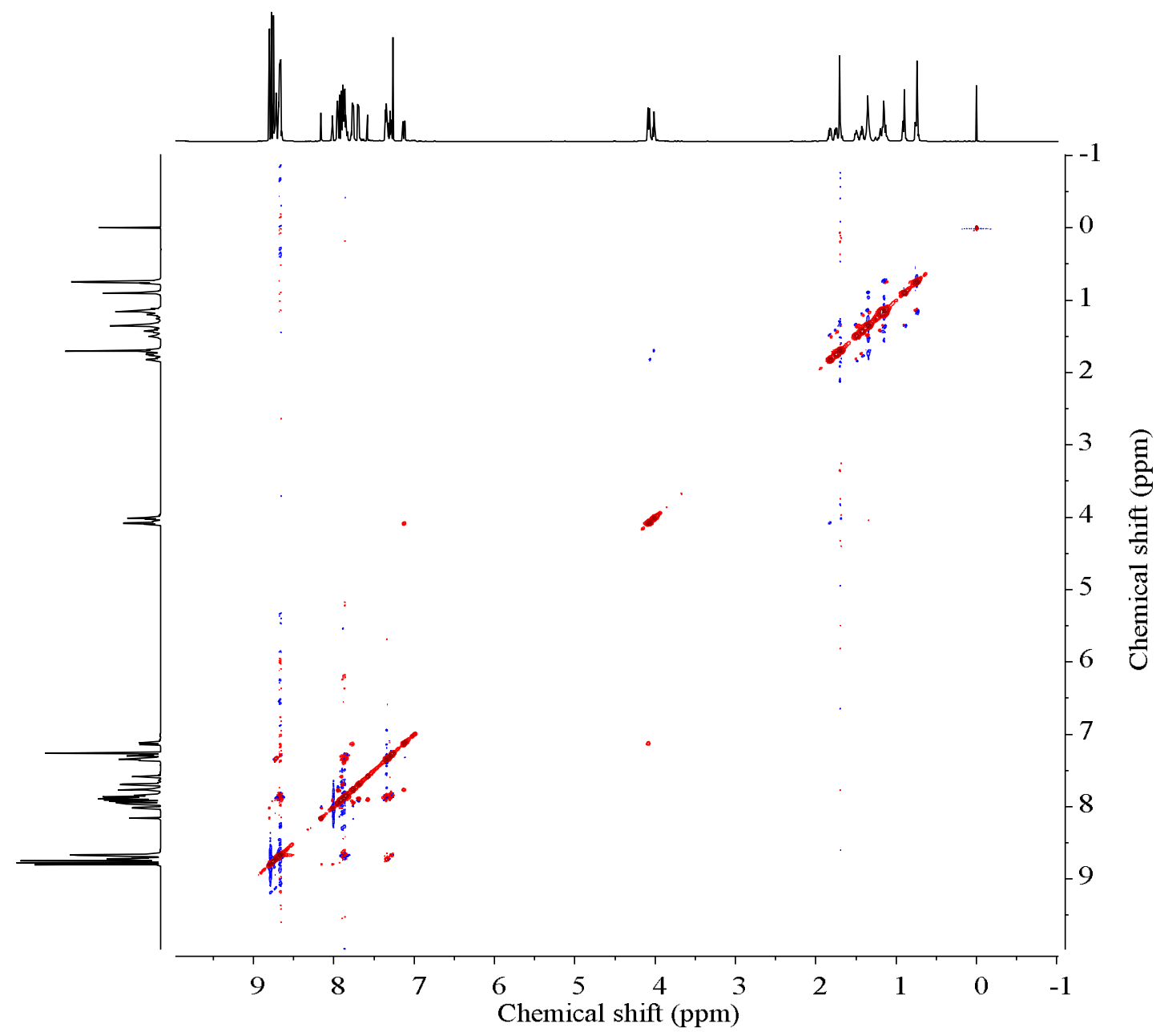

Figure S137. 2D NOESY NMR (600 MHz, $\left.\mathrm{CDCl}_{3}\right)$ spectrum of ligand $\mathbf{L B}$.

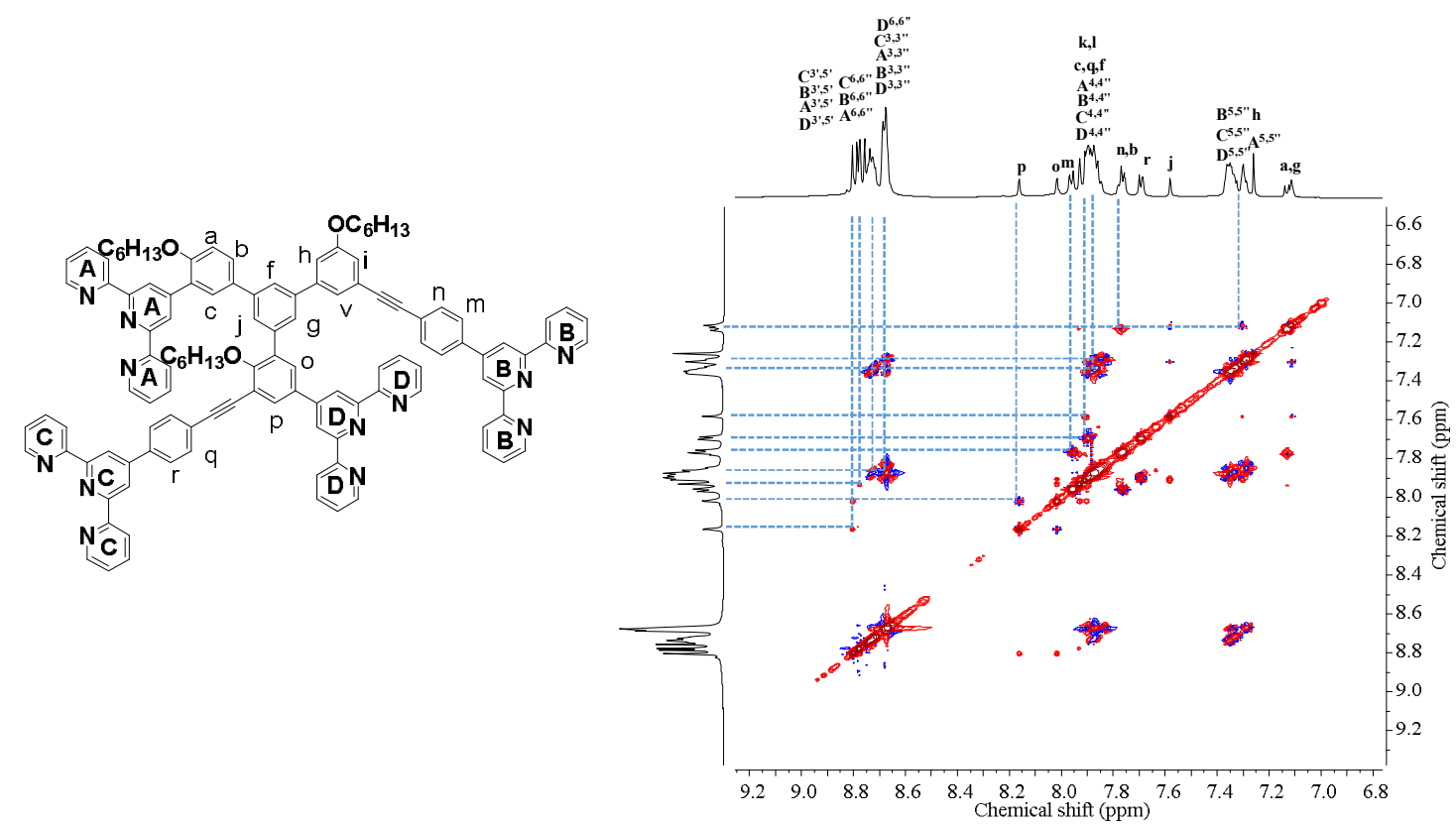

Figure S138. 2D NOESY NMR (600 $\left.\mathrm{MHz}, \mathrm{CDCl}_{3}\right)$ spectrum of ligand $\mathbf{L B}$ (aromatic region). 


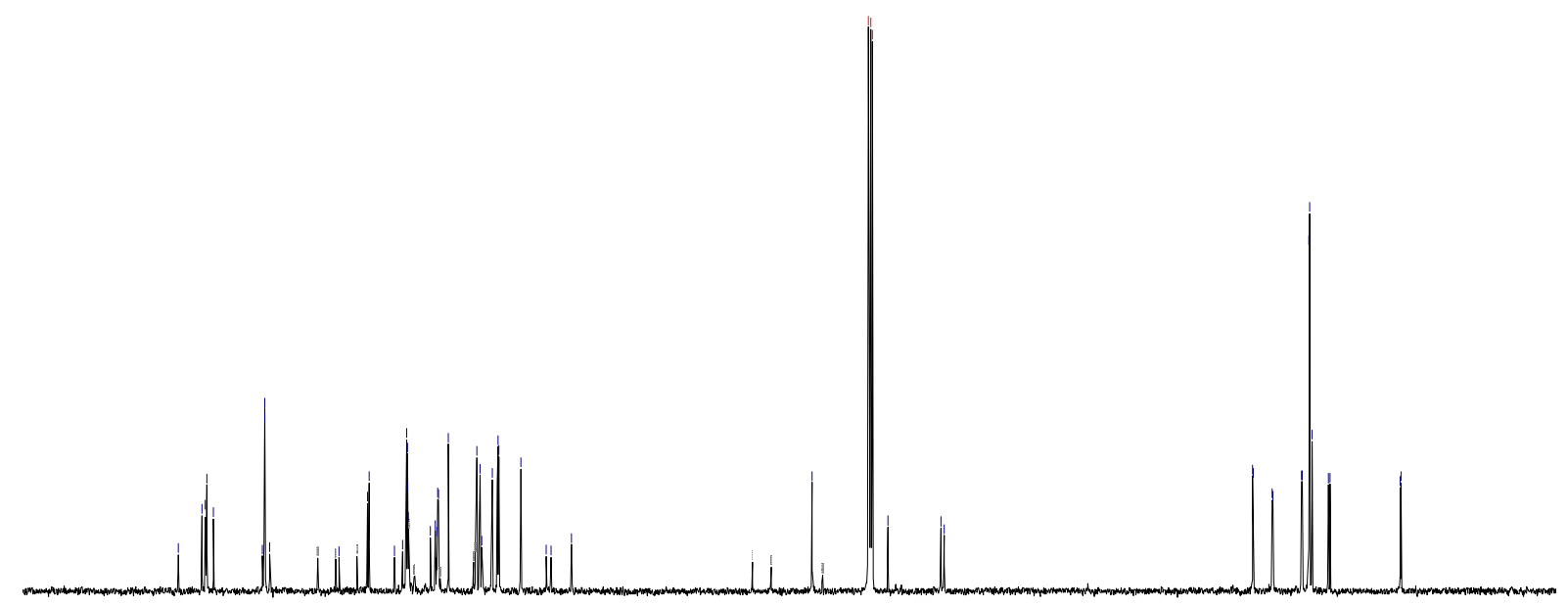

$\begin{array}{llllllllllllllllll}170 & 160 & 150 & 140 & 130 & 120 & 110 & \begin{array}{l}100 \\ \text { Chemical shift (ppm) }\end{array}\end{array}$

Figure S139. ${ }^{13} \mathrm{C} \mathrm{NMR}\left(150 \mathrm{MHz}, \mathrm{CDCl}_{3}\right)$ spectrum of ligand $\mathbf{L B}$.

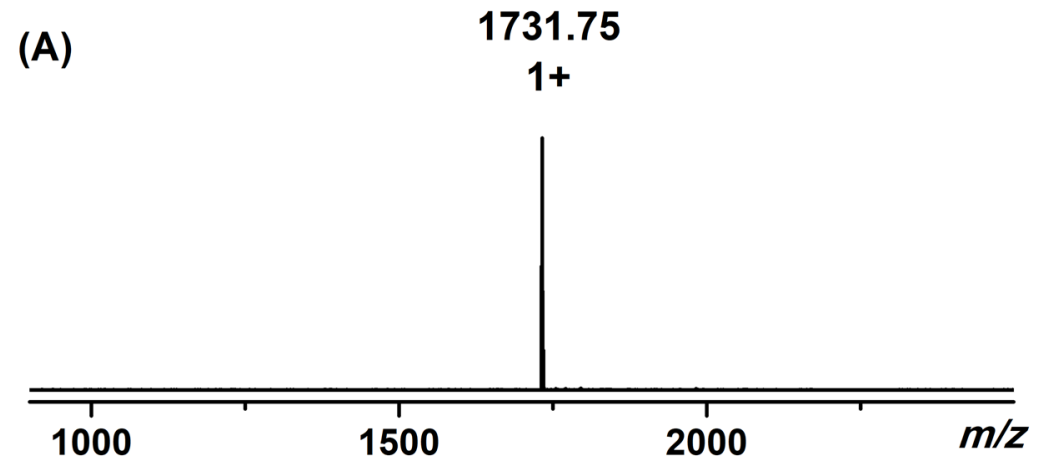

(B)

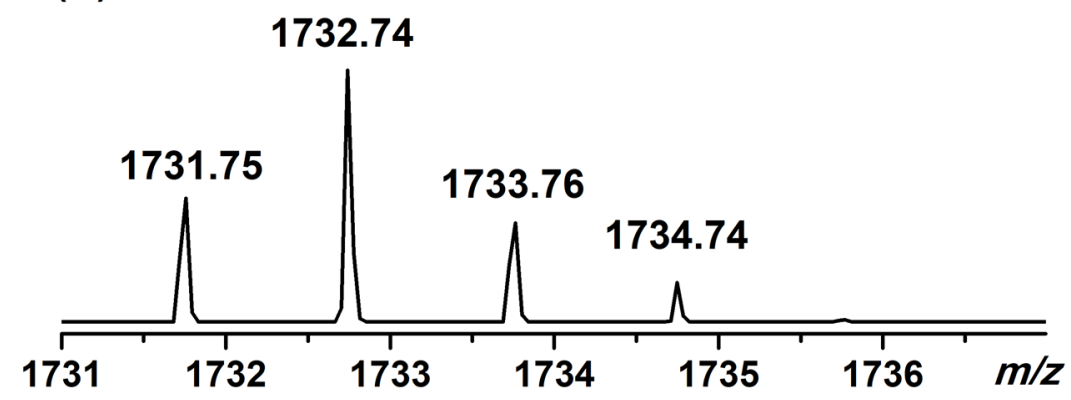

Figure S140. (A) MALDI-TOF spectrum of ligand $\mathbf{L B}$ in $\mathrm{CHCl}_{3} / \mathrm{CH}_{3} \mathrm{OH}(1 / 3)$ and (B) 
isotope pattern.

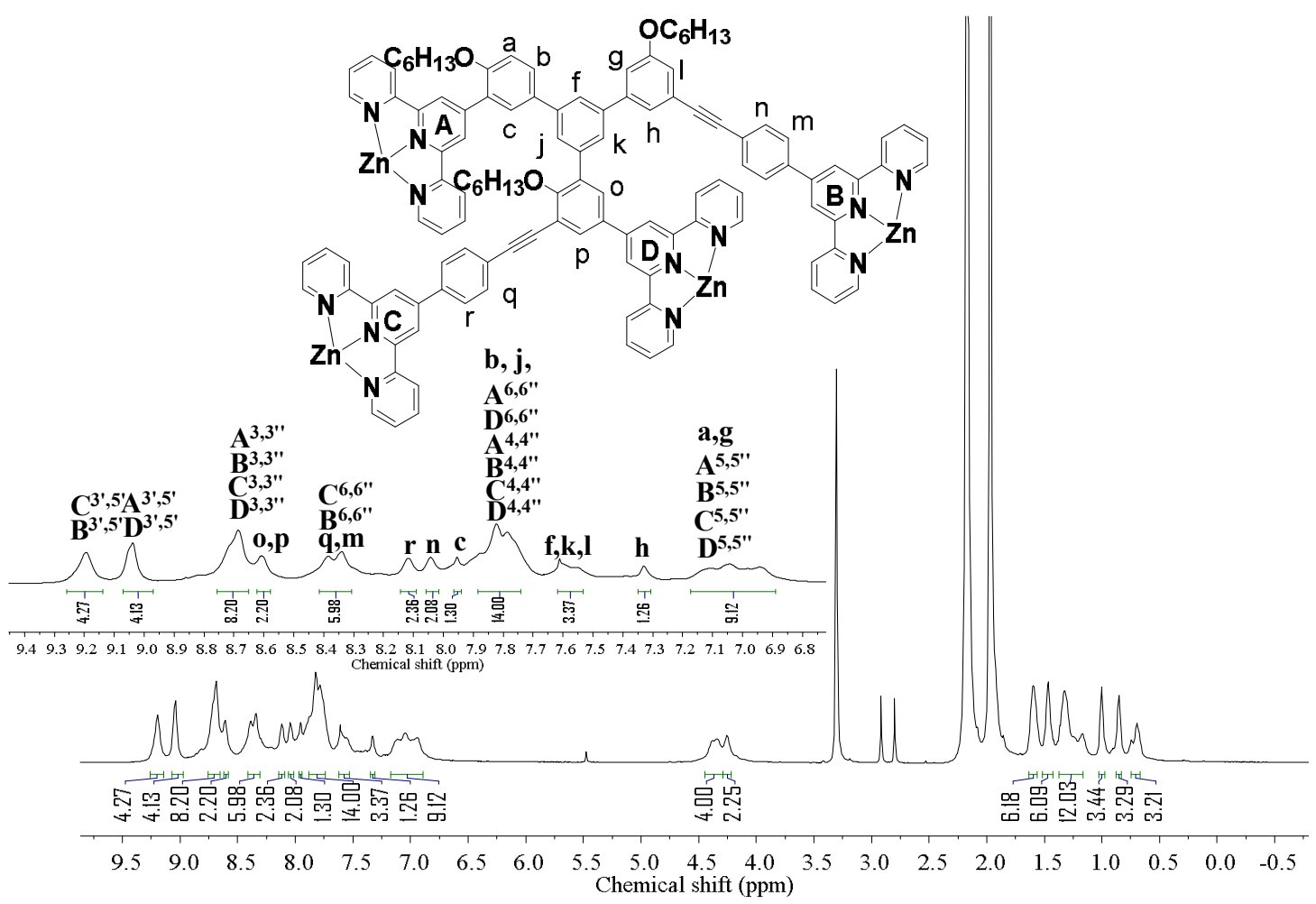

Figure S141. ${ }^{1} \mathrm{H}$ NMR $\left(600 \mathrm{MHz}, \mathrm{CD}_{3} \mathrm{CN}\right)$ spectrum of complexes $\mathbf{B}$.

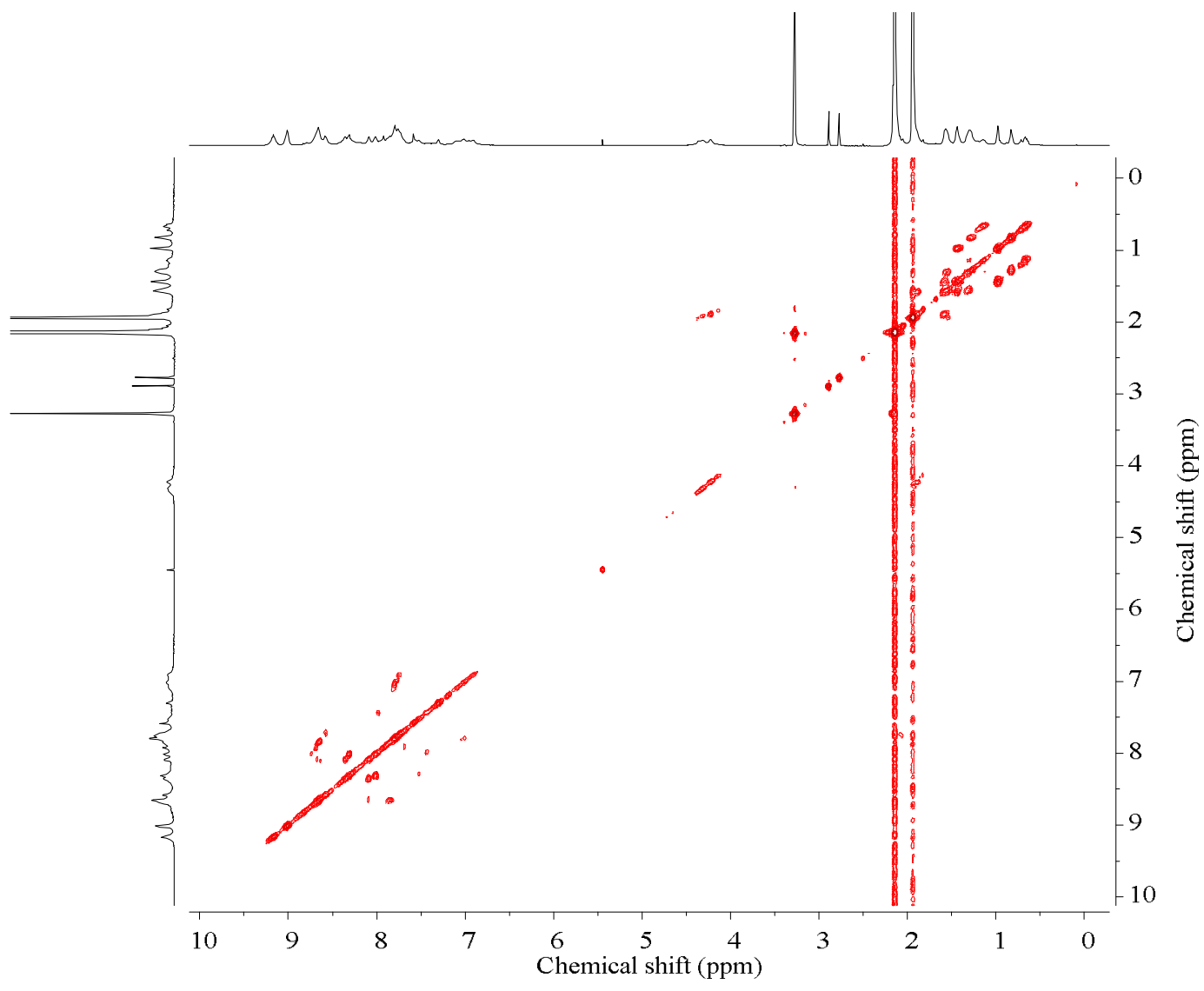


Figure S142. 2D COSY NMR (600 MHz, $\left.\mathrm{CD}_{3} \mathrm{CN}\right)$ spectrum of complexes B.

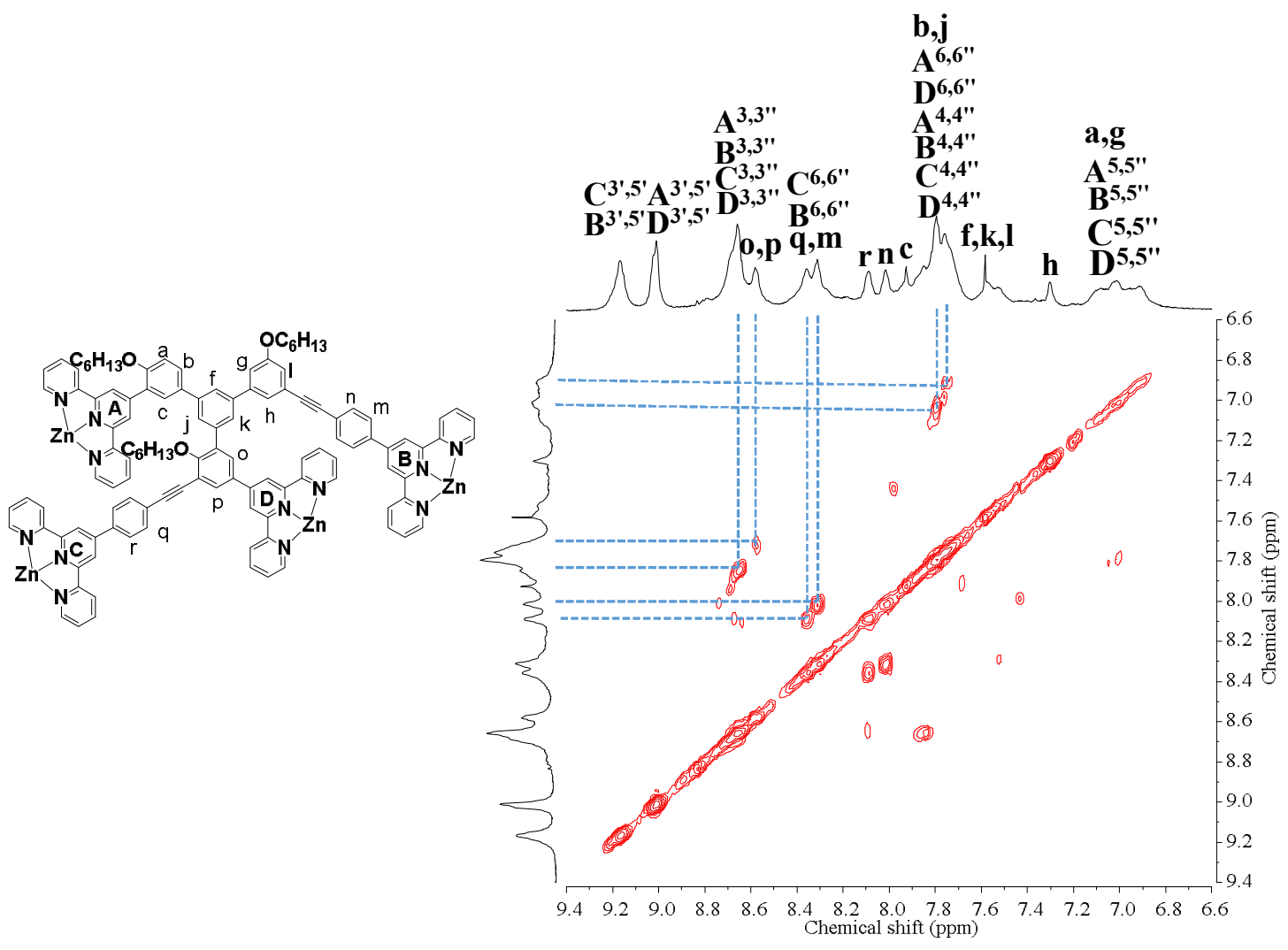

Figure S143. 2D COSY NMR (600 MHz, $\left.\mathrm{CD}_{3} \mathrm{CN}\right)$ spectrum of complexes $\mathbf{B}$ (aromatic region).

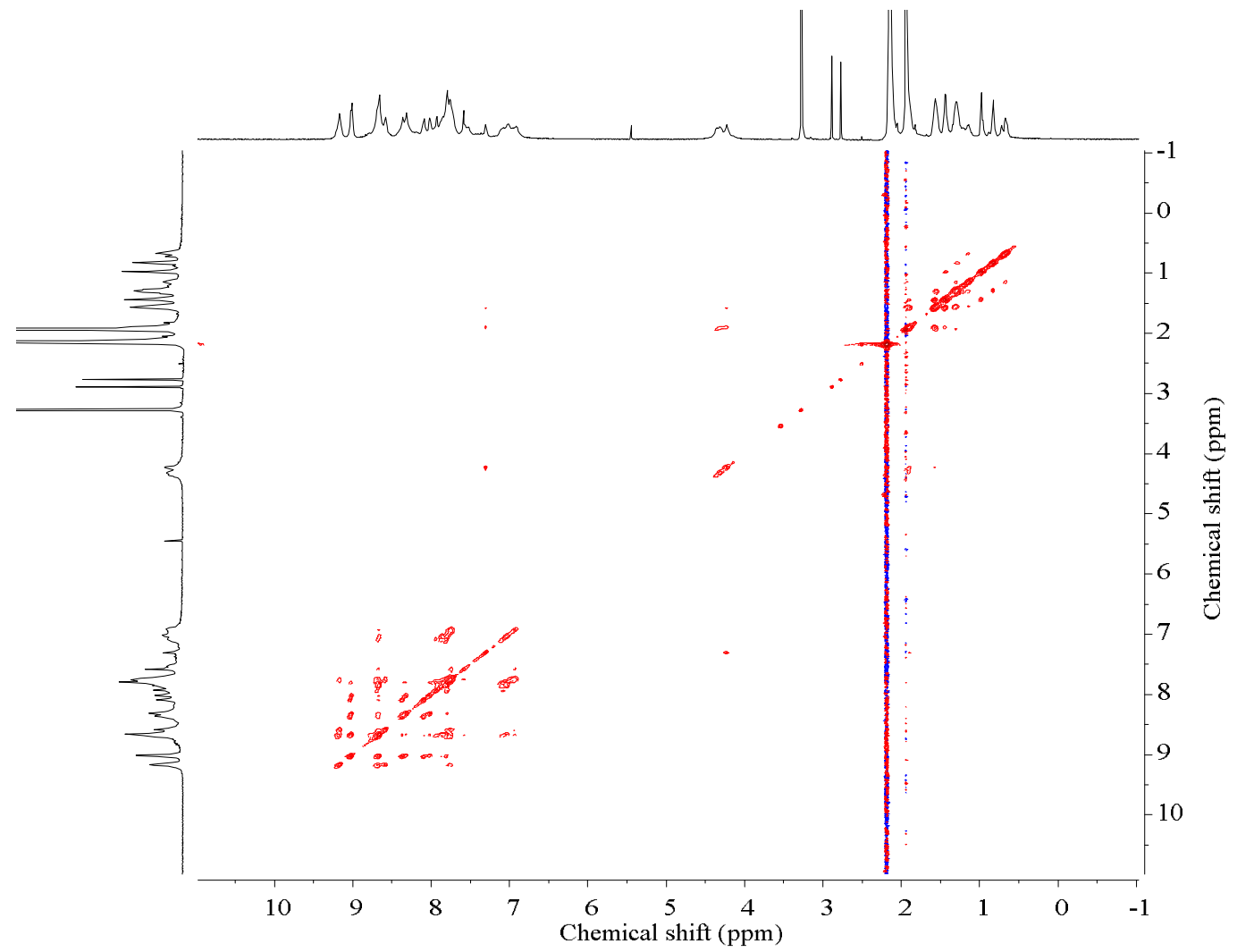

Figure S144. 2D NOESY NMR (600 MHz, $\left.\mathrm{CD}_{3} \mathrm{CN}\right)$ spectrum of complexes $\mathbf{B}$. 


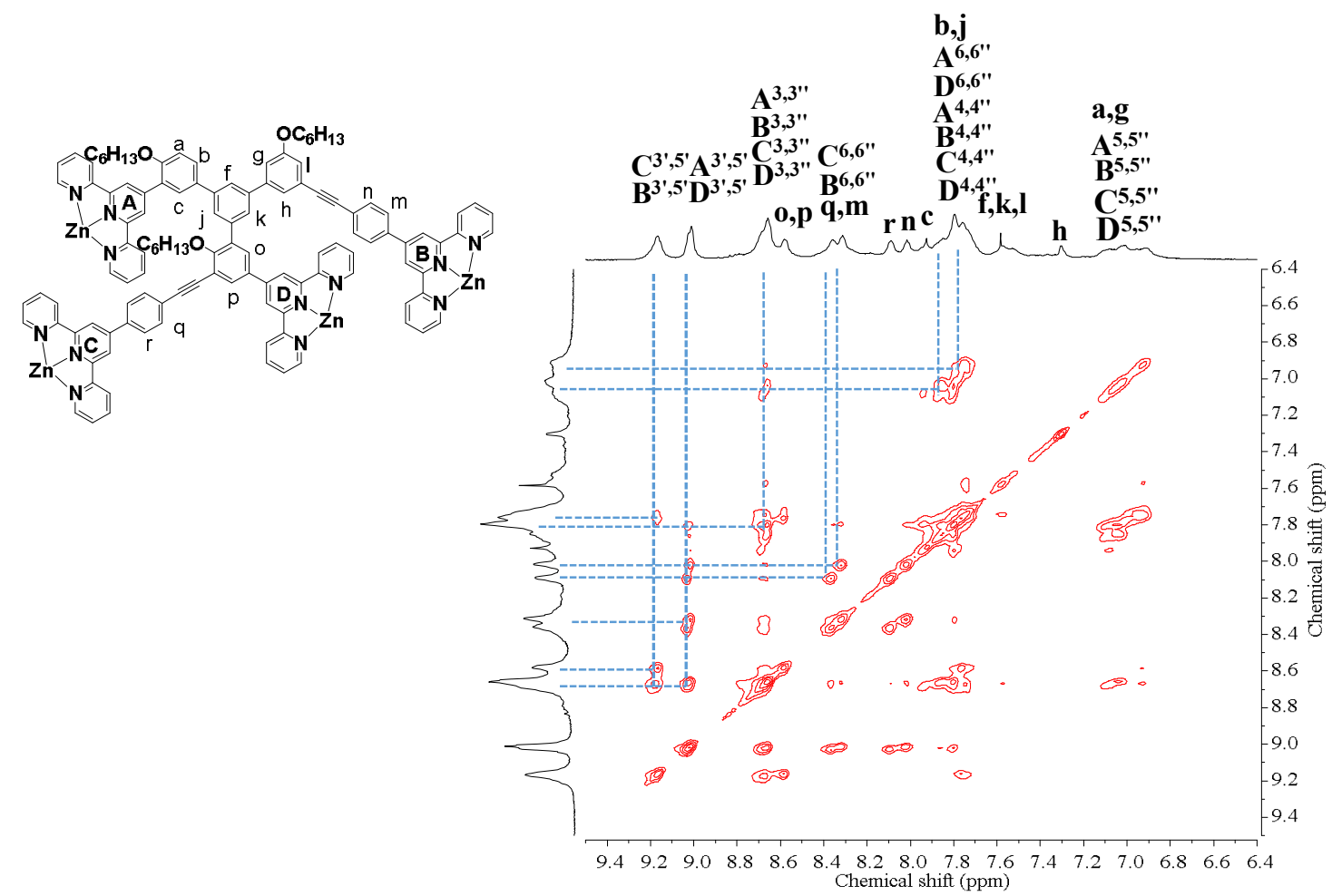

Figure S145. 2D NOESY NMR (600 MHz, $\left.\mathrm{CD}_{3} \mathrm{CN}\right)$ spectrum of complexes B (aromatic region).

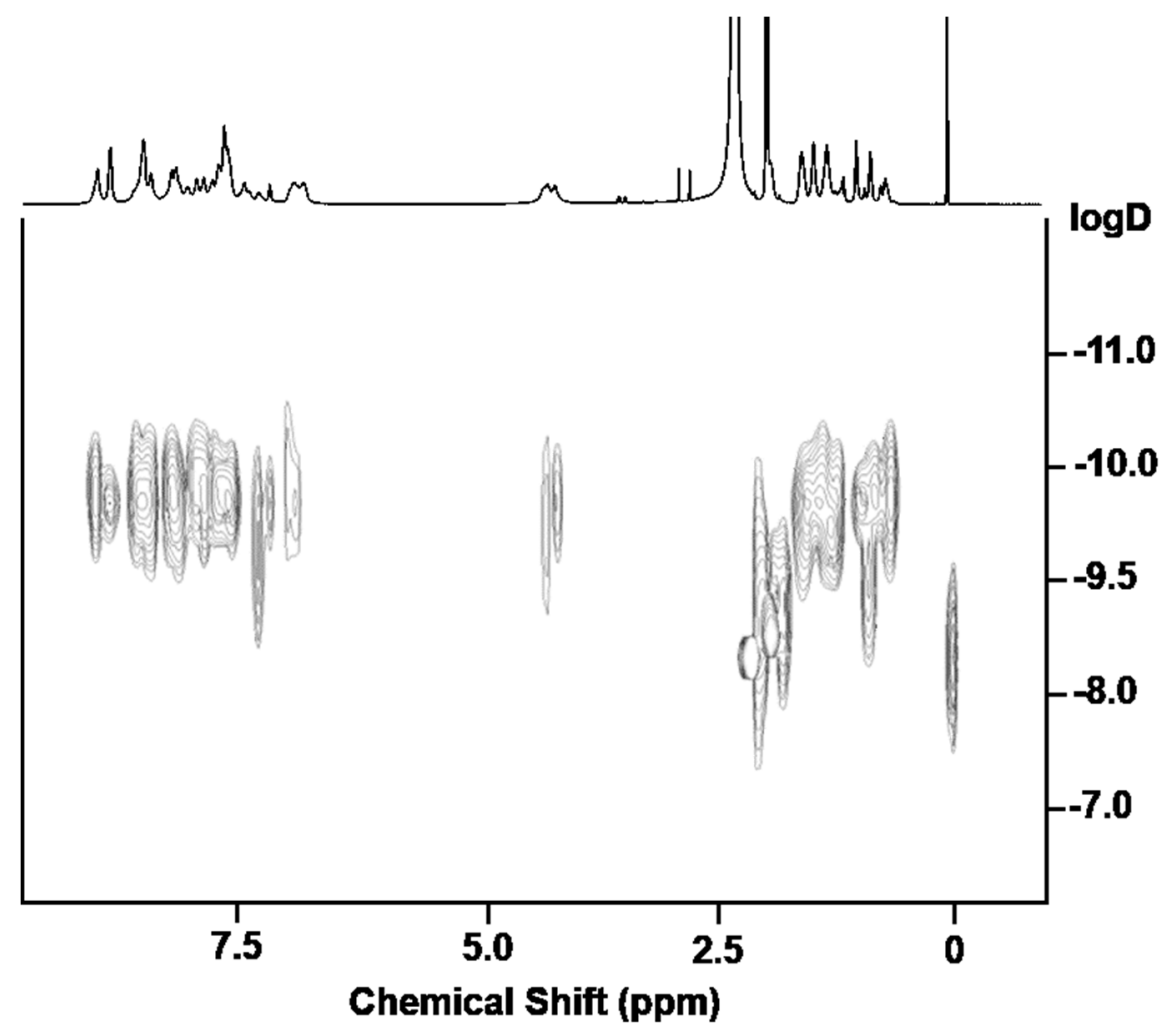

Figure S146. DOSY NMR (500 MHz, $\left.\mathrm{CD}_{3} \mathrm{CN}\right)$ spectrum of complexes B. 


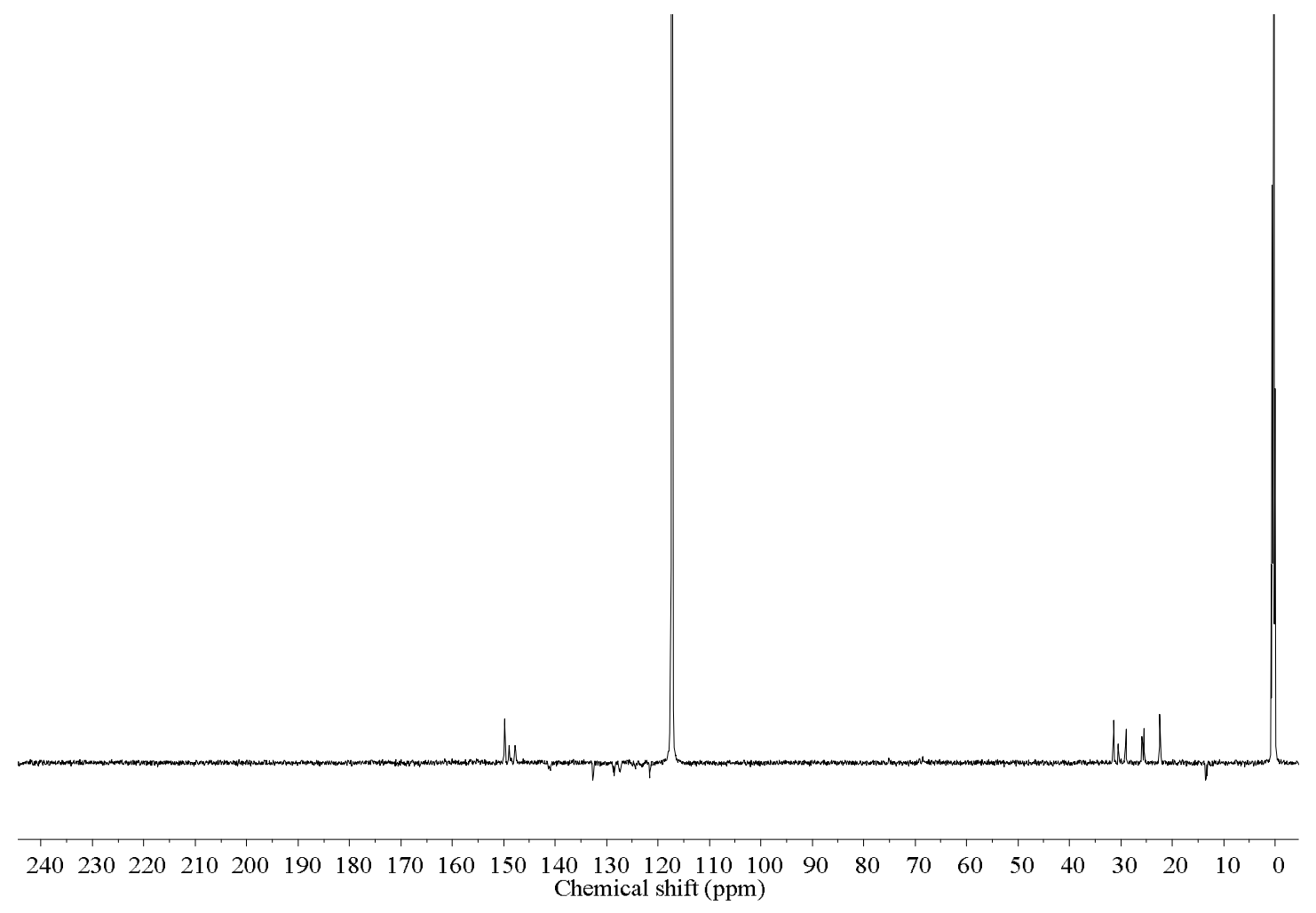

Figure S147. ${ }^{13} \mathrm{C}$ NMR $\left(150 \mathrm{MHz}, \mathrm{CD}_{3} \mathrm{CN}\right)$ spectrum of complexes $\mathbf{B}$.

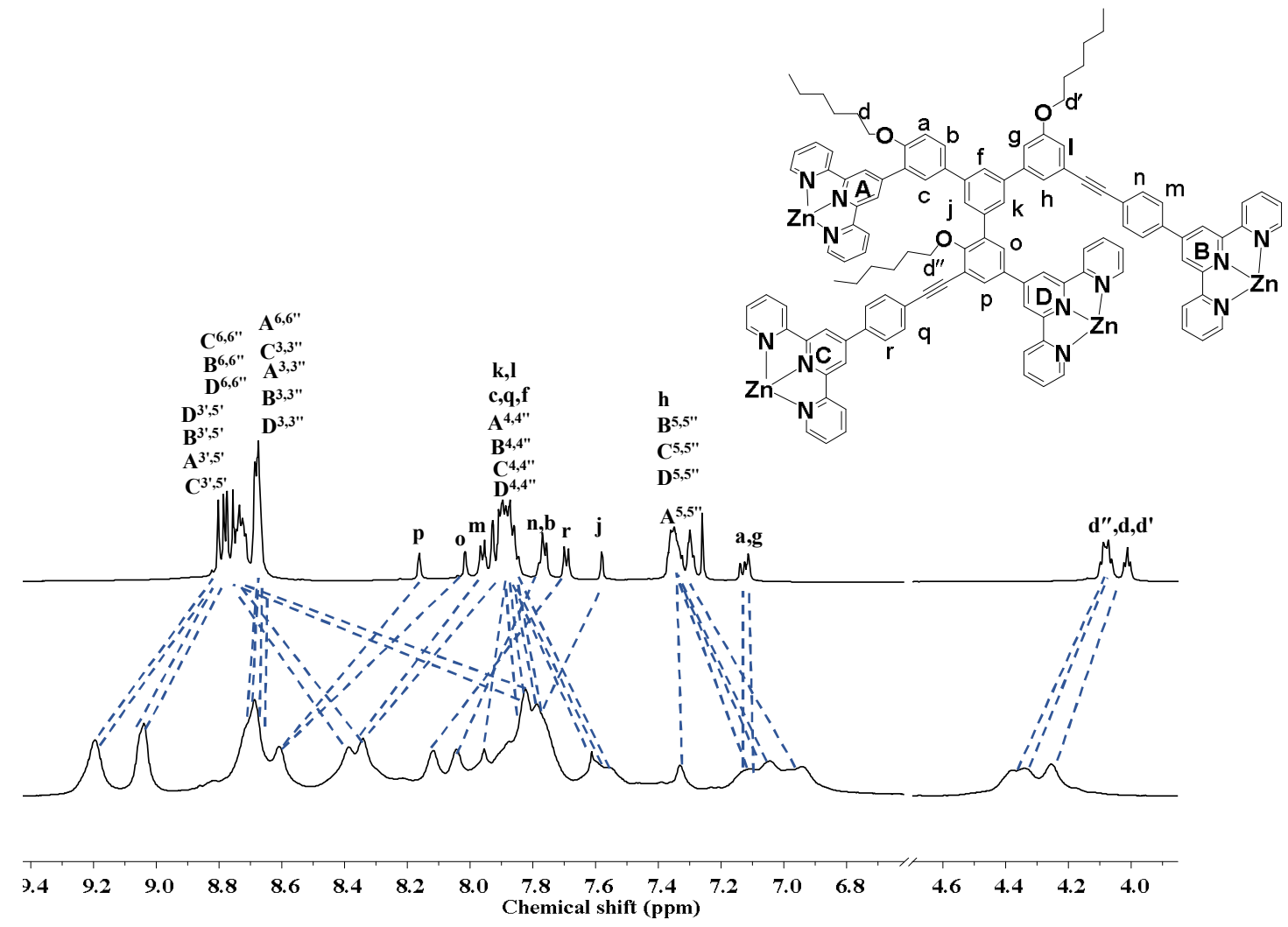

Figure S148. ${ }^{1} \mathrm{H}$ NMR spectra $(600 \mathrm{MHz})$ of (A) ligand $\mathbf{L B}$ in $\mathrm{CDCl}_{3}$ and (B) complexes $\mathbf{B}$ in $\mathrm{CD}_{3} \mathrm{CN}$. 


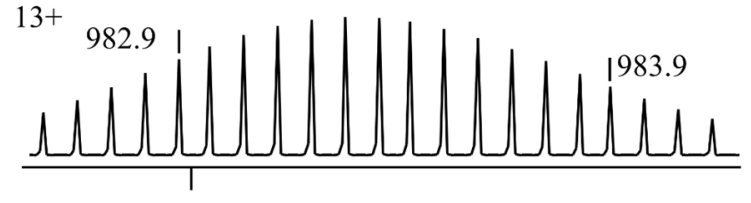
$\frac{{ }_{982}^{1}}{N_{m / z}^{982.9}}$

$15+$
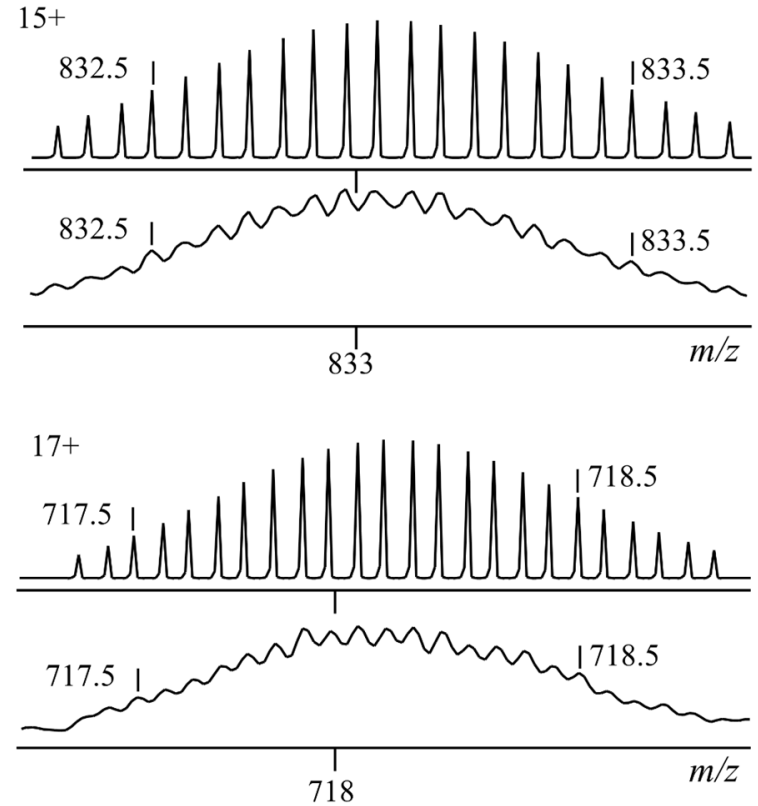
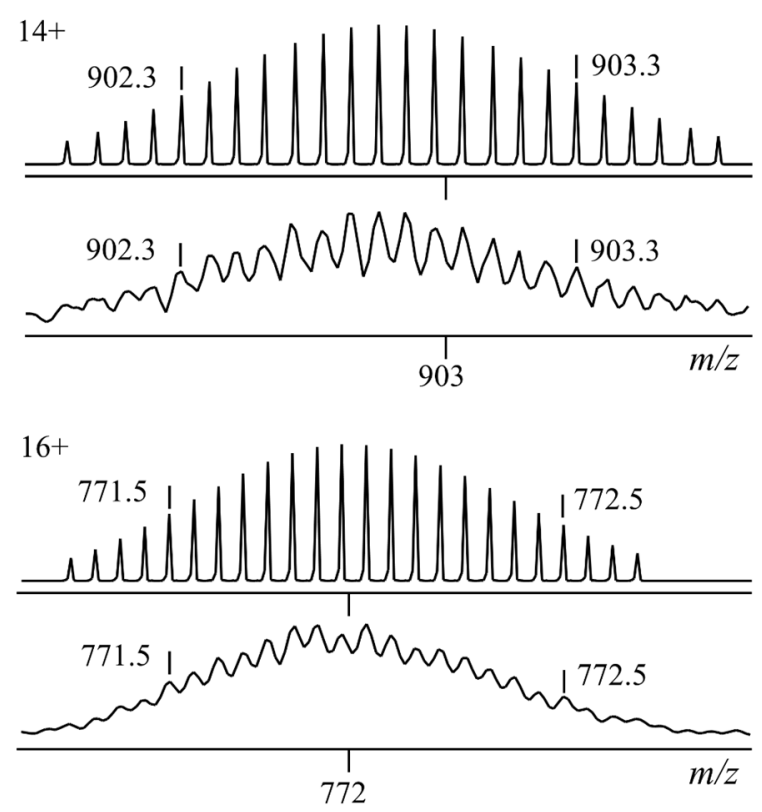

Figure S149. Measured (bottom) and calculated (top) isotope patterns for different charge states observed from hexamer $\mathbf{B} 1$ or $\mathbf{Z n}_{12} \mathbf{L B}_{\mathbf{6}}\left(\mathrm{PF}_{6}^{-}\right.$as counterion). 
$15+$
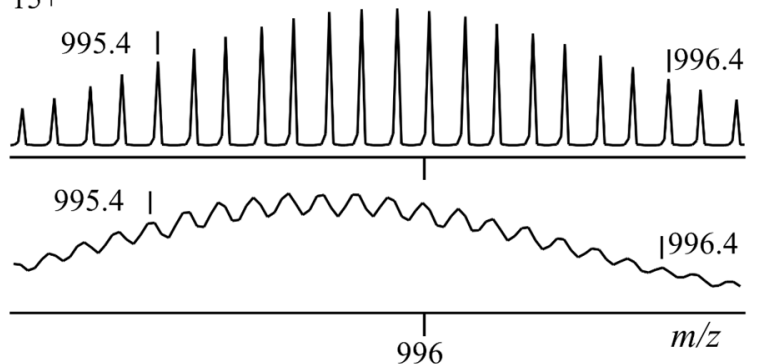

$17+$

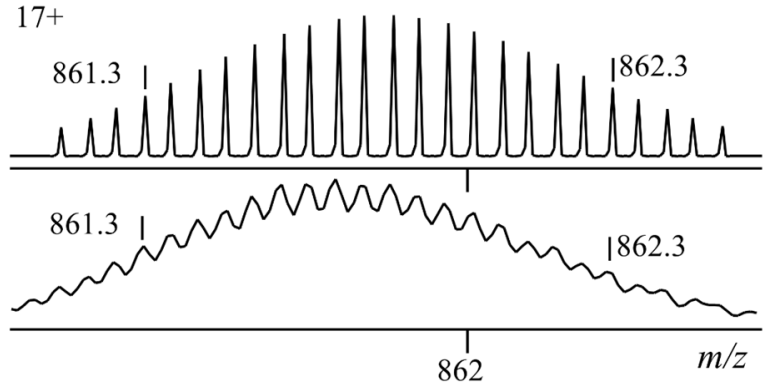

$19+$

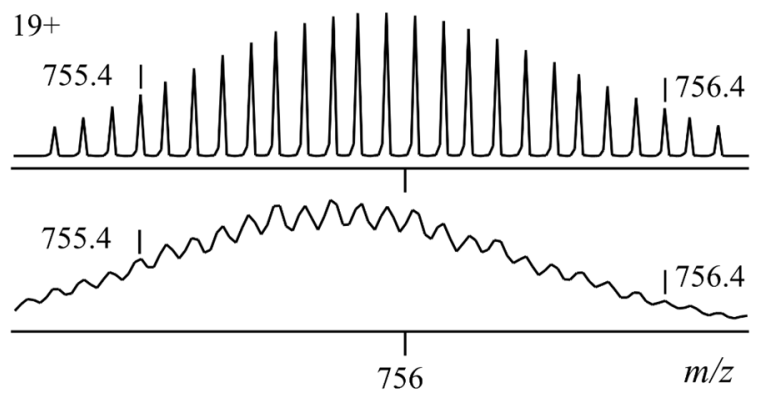

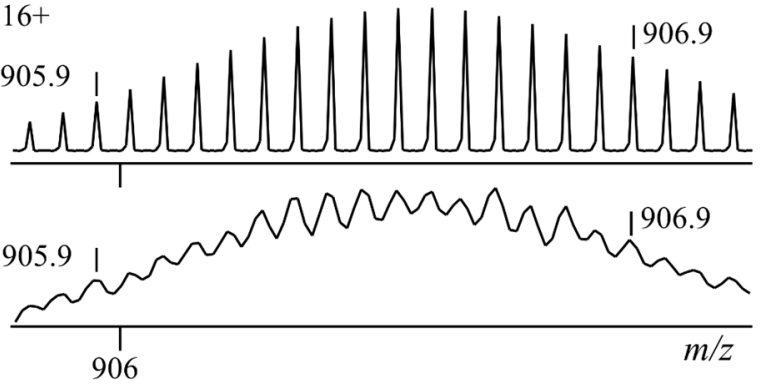

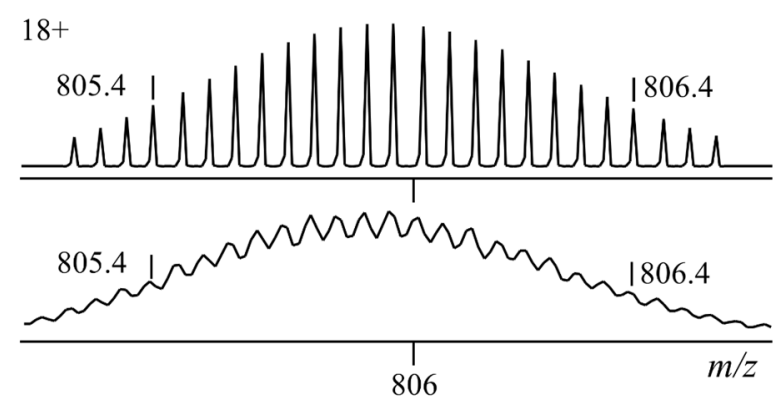

$20+$

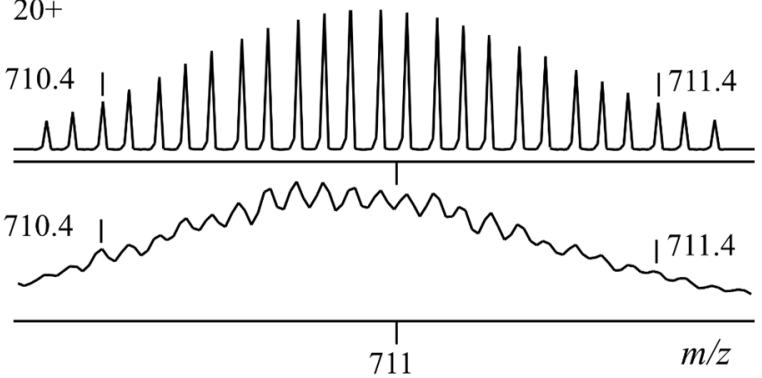

Figure S150. Measured (bottom) and calculated (top) isotope patterns for different charge states observed from heptamer $\mathbf{B} 2$ or $\mathbf{Z n}_{14} \mathbf{L B}_{7}\left(\mathrm{PF}_{6}^{-}\right.$as counterion). 
$11+$

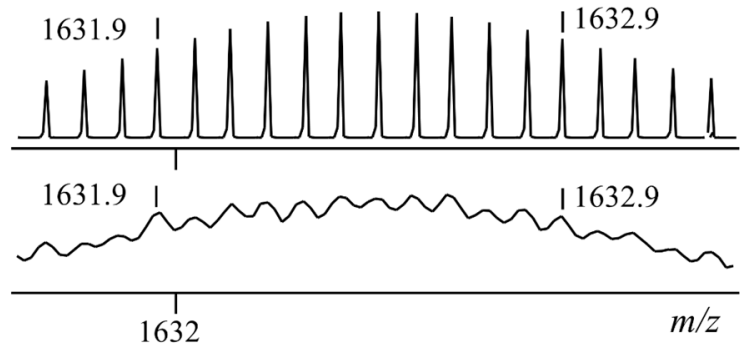

$13+$
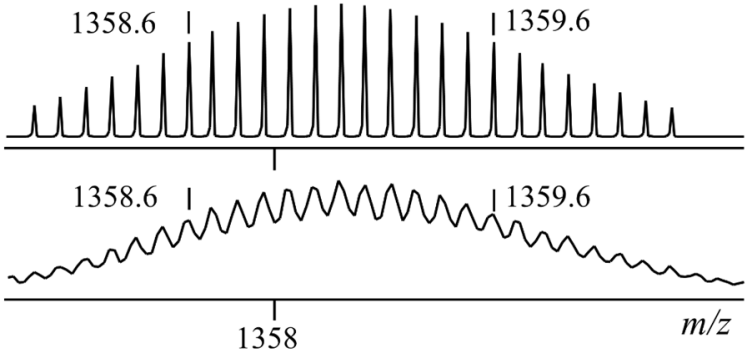

$16+$

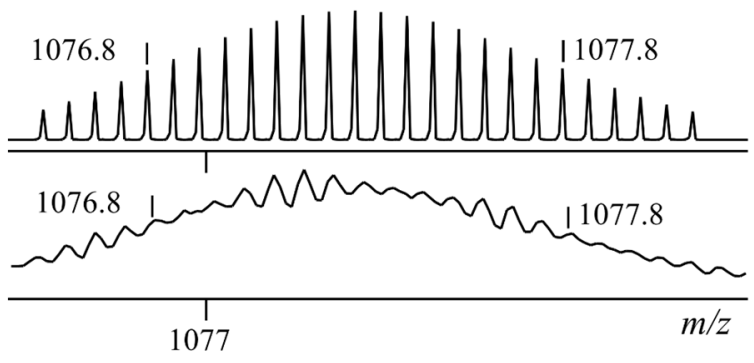

$18+$

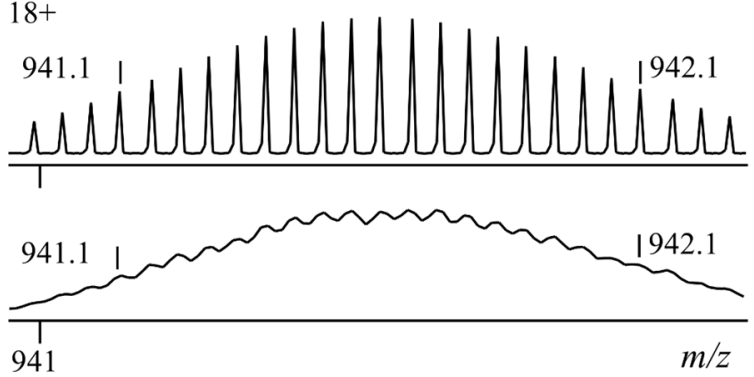

$21+$
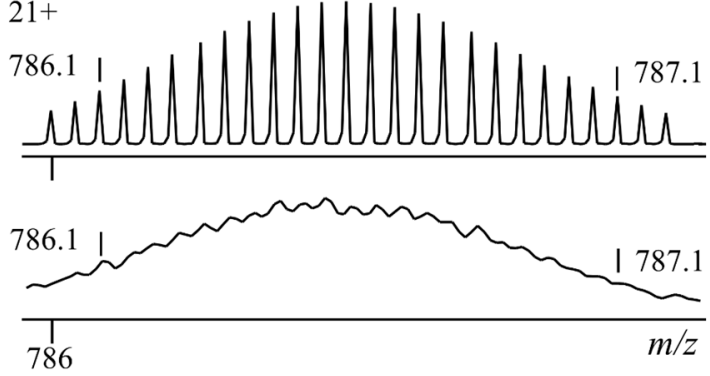

$12+$

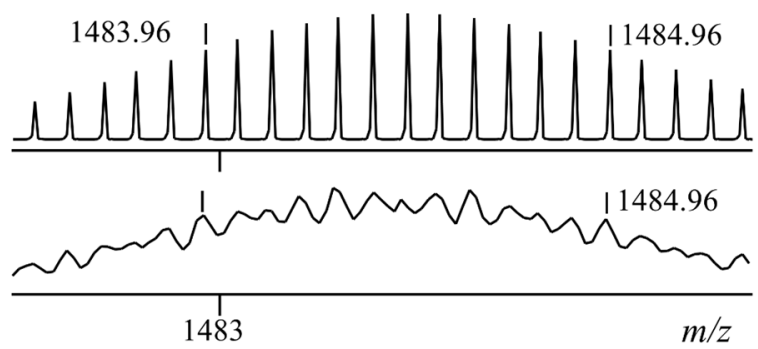

$15+$

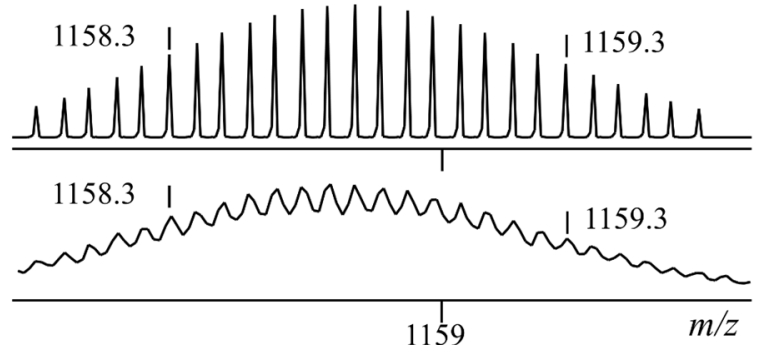

$17+$
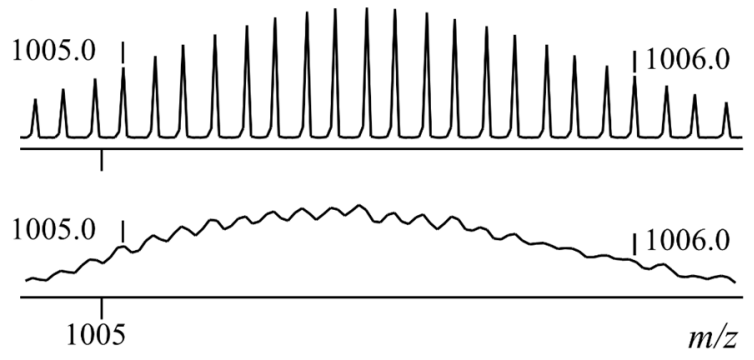

$19+$
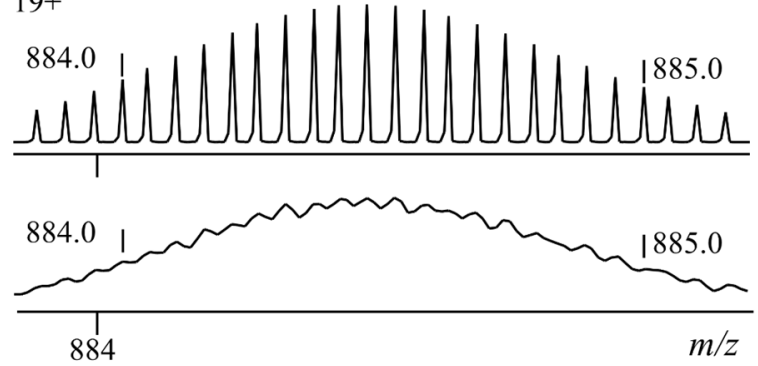

Figure S151. Measured (bottom) and calculated (top) isotope patterns for different charge states observed from octamer $\mathbf{B} 3$ or $\mathbf{Z n}_{\mathbf{1 6}} \mathbf{L B}_{\mathbf{8}}$ ( $\mathrm{PF}_{6}^{-}$as counterion). 


\section{UV-Vis Spectra of Ligands and Complexes}
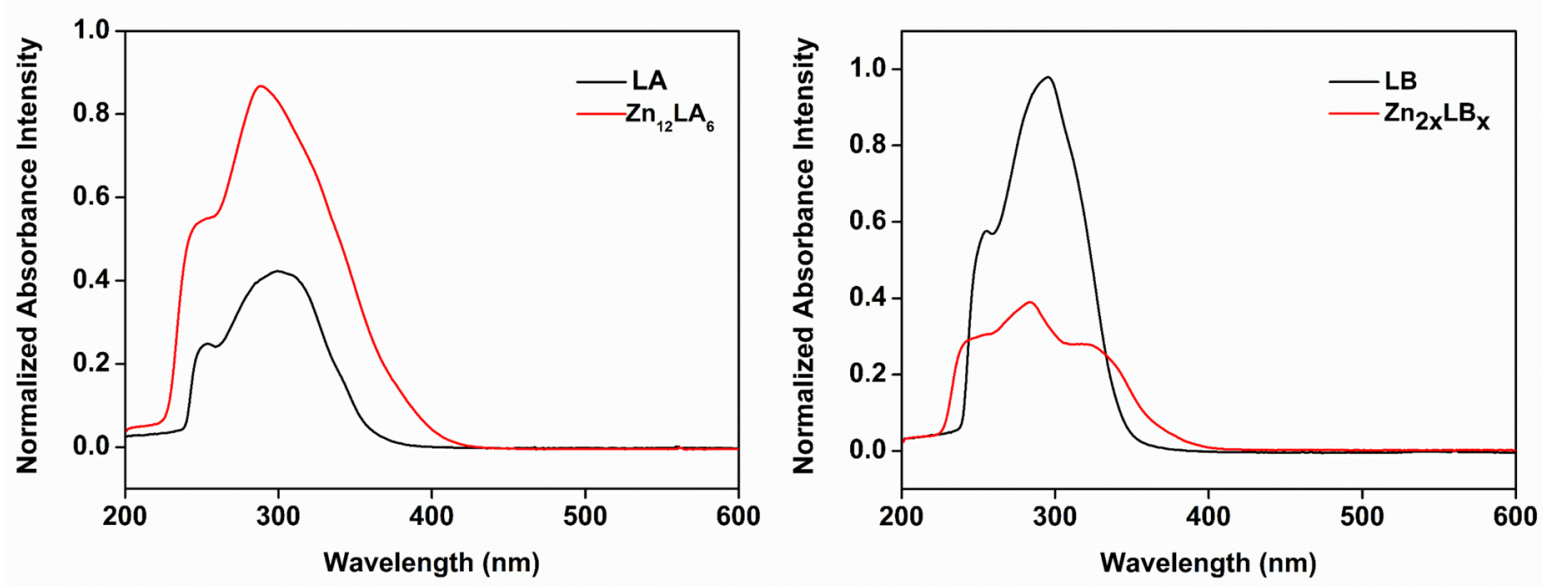

Figure S152. UV-Vis absorption spectra of ligands in $\mathrm{CHCl}_{3}$ and complexes in $\mathrm{CH}_{3} \mathrm{CN}$.

\section{DFT Calculations on Binding Energies of A1-A4}

To address the distribution of isomers A1-A4, we estimated the binding (intermolecular interaction) energies of A1-A4 by the model system. The structures of entire supramolecular system (hexamer) were too large to calculate directly. The model system was chosen to simplify the calculation and estimate the total binding energy of each supramolecular isomer. And the model systems were shown in Figure S153. The binding energies $\left(E_{b}\right)$ are as follows:

\section{A B}

$E_{b}=E_{A B}-E_{A}-E_{B}$

$\mathrm{E}_{\mathrm{AB}}$ : total energy of a unit.

$\mathrm{E}_{\mathrm{A}}$ and $\mathrm{E}_{\mathrm{B}}$ : energy of left and right parts.

$\mathrm{E}_{\mathrm{b}}$ : binding energy of $\mathrm{Zn}(\mathrm{II})$ and terpyridine.

\section{LA-1:}

$\mathrm{E}_{\mathrm{b} 1}=-6597.18615148-(-3232.98592112)-(-3232.98592112)=-131.2144462($ Hartree $)$

\section{LA-2:}

$\mathrm{E}_{\mathrm{b} 2}=-5677.82090642-(-3232.98278665)-(-2313.62918253)=-131.2089372$ (Hartree) 


\section{LA-3:}

$\mathrm{E}_{\mathrm{b} 3}=-4758.45759335-(-2313.62873693)-(-2313.62873689)=-131.2001195$ (Hartree)

\section{LA-4:}

$E_{\mathrm{b} 4}=-5677.81643112-(-2618.78358833)-(-2927.82612946)=-131.2067133$ (Hartree)

The binding energies of A1-A4 can be estimated as:

$\mathrm{E}_{\mathrm{A} 1}=6 \times \mathrm{E}_{\mathrm{b} 2}=6 \times(-131.2089372)=-787.2536232($ Hartree $)$

$\mathrm{E}_{\mathrm{A} 2}=6 \times \mathrm{E}_{\mathrm{b} 4}=6 \times(-131.2067133)=-787.2402798($ Hartree $)$

$\mathrm{E}_{\mathrm{A} 3}=3 \times \mathrm{E}_{\mathrm{b} 1}+3 \times \mathrm{E}_{\mathrm{b} 3}=3 \times(-131.2144462)+3 \times(-131.2001195)=-787.2436971$ (Hartree)

$\mathrm{E}_{\mathrm{A} 4}=2 \times \mathrm{E}_{\mathrm{b} 1}+2 \times \mathrm{E}_{\mathrm{b} 2}+2 \times \mathrm{E}_{\mathrm{b} 3}=2 \times(-131.2144462)+2 \times(-131.2089372)+2 \times(-131.2001195)=-787.24$ 70058 (Hatree)

From the calculated total binding energies of A1-A4, we find that A1 possesses the highest binding energy, corresponding to the most stable structure; whereas, A2 with the lowest binding energy could be the most unstable structure. As such, the stabilities of A1-A4 are in the order of $\mathrm{E}_{\mathrm{A} 1}>\mathrm{E}_{\mathrm{A} 4}>\mathrm{E}_{\mathrm{A} 3}>\mathrm{E}_{\mathrm{A} 2}$, which is consistent with the experimental results. Note that Hartree is an energy unit of HF (Hartree-Fock), 1 Hartree $=2625.5 \mathrm{~kJ} / \mathrm{mol}$.

Table S1. Binding energy for isomers given by DFT calculation

\begin{tabular}{ccc}
\hline Isomer & Coordination type & Binding energy(Hartree) \\
\hline $\mathrm{A} 1$ & $6 \times \mathrm{E}_{\mathrm{b} 2}$ & -787.2536232 \\
$\mathrm{~A} 2$ & $6 \times \mathrm{E}_{\mathrm{b} 4}$ & -787.2402798 \\
$\mathrm{~A} 3$ & $3 \times \mathrm{E}_{\mathrm{b} 1}+3 \times \mathrm{E}_{\mathrm{b} 3}$ & -787.2436971 \\
$\mathrm{~A} 4$ & $2 \times \mathrm{E}_{\mathrm{b} 1}+2 \times \mathrm{E}_{\mathrm{b} 2}+2 \times \mathrm{E}_{\mathrm{b} 3}$ & -787.2470058 \\
\hline
\end{tabular}


A
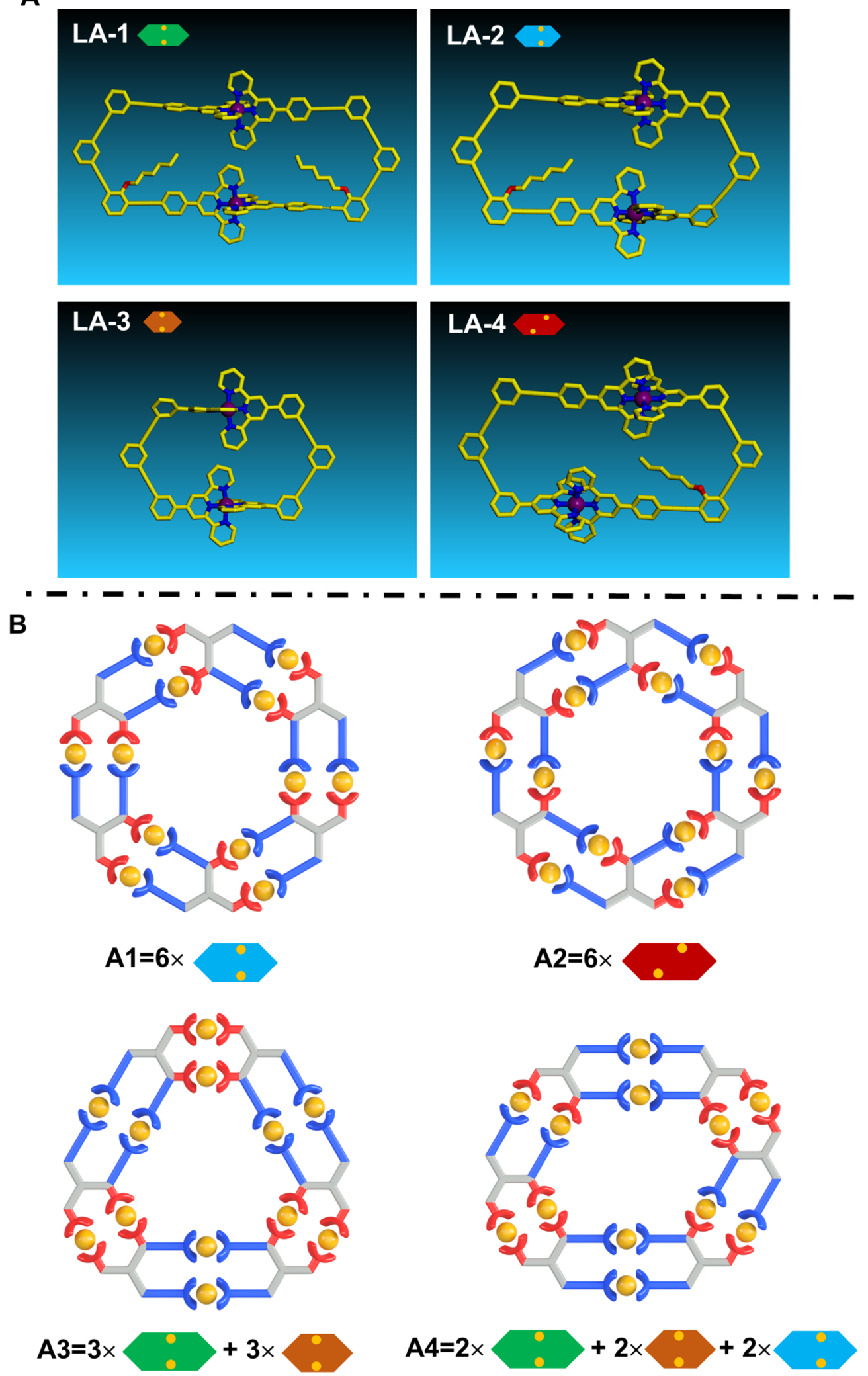

Figure S153. (A) Four binding modes of $\mathbf{Z n}_{2} \mathbf{L} \mathbf{A}_{2}$ model system (Red-O, Yellow-C, Blue-N, Purple-Zn) and (B) isomers' compositions of supramolecular system. 


\section{STM Data}
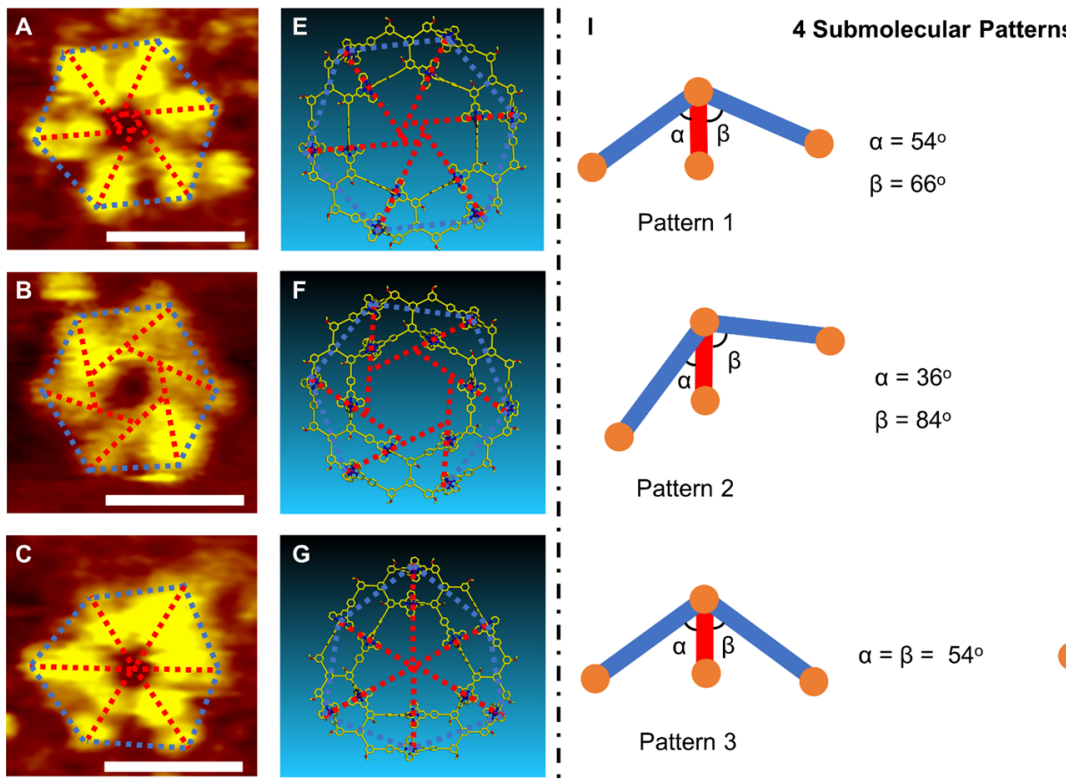

Pattern 3

$\alpha=\beta=54^{\circ}$
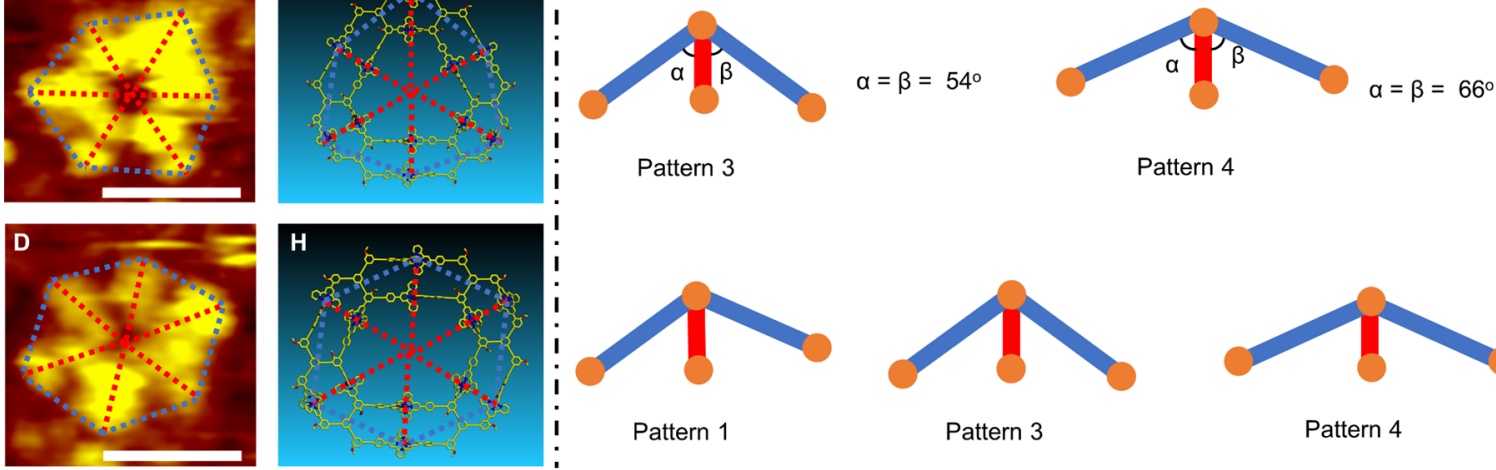

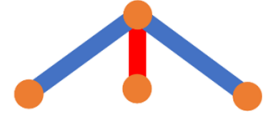

Pattern 3

Figure S154. (A), (B), (C) and (D) STM images of supramolecules A1, A2, A3 and A4 with different patterns labeled, scale bar $5 \mathrm{~nm}$. (E), (F), (G) and (H) Representative energy-minimized structures from molecular modeling of complexes A1, A2, A3 and A4 with patterns labeled. Alkyl chains were replaced by methoxy for clarity. (I) Four types of patterns found in STM images.
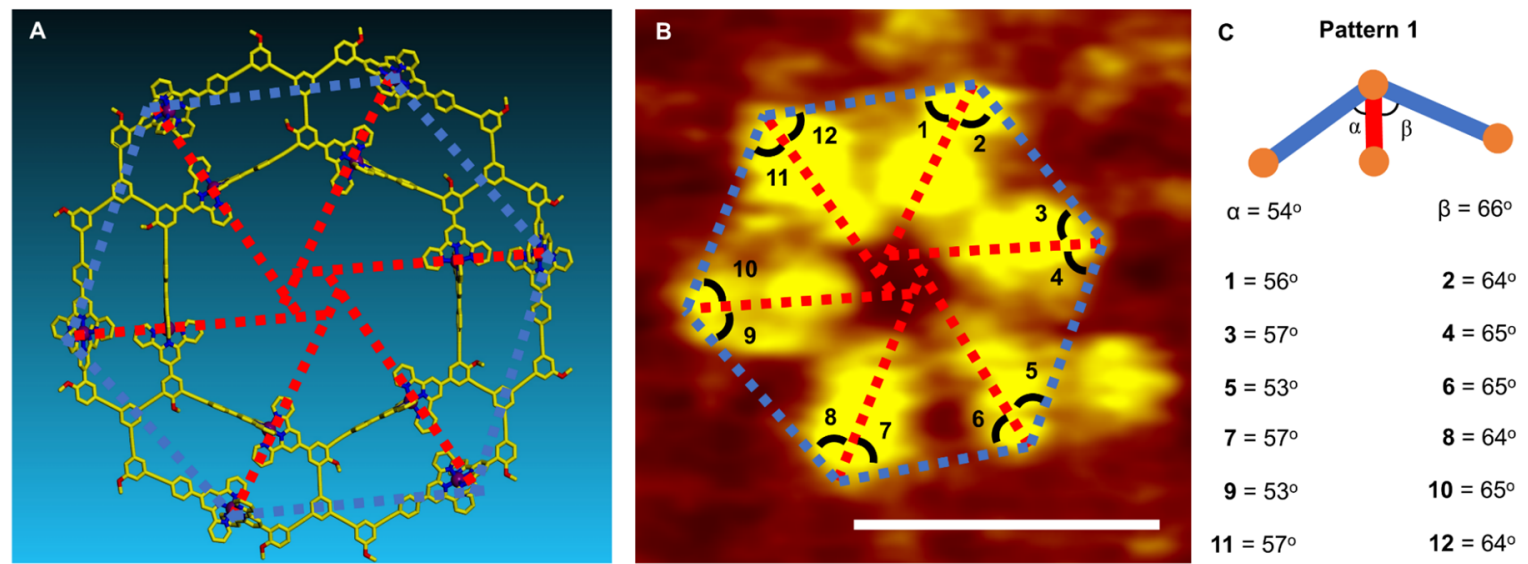

Figure S155. The comparison of (A) modeling structure and (B) STM image of isomer A1 with pattern labeled and measured. (C) Angles of the pattern measured in STM image. 

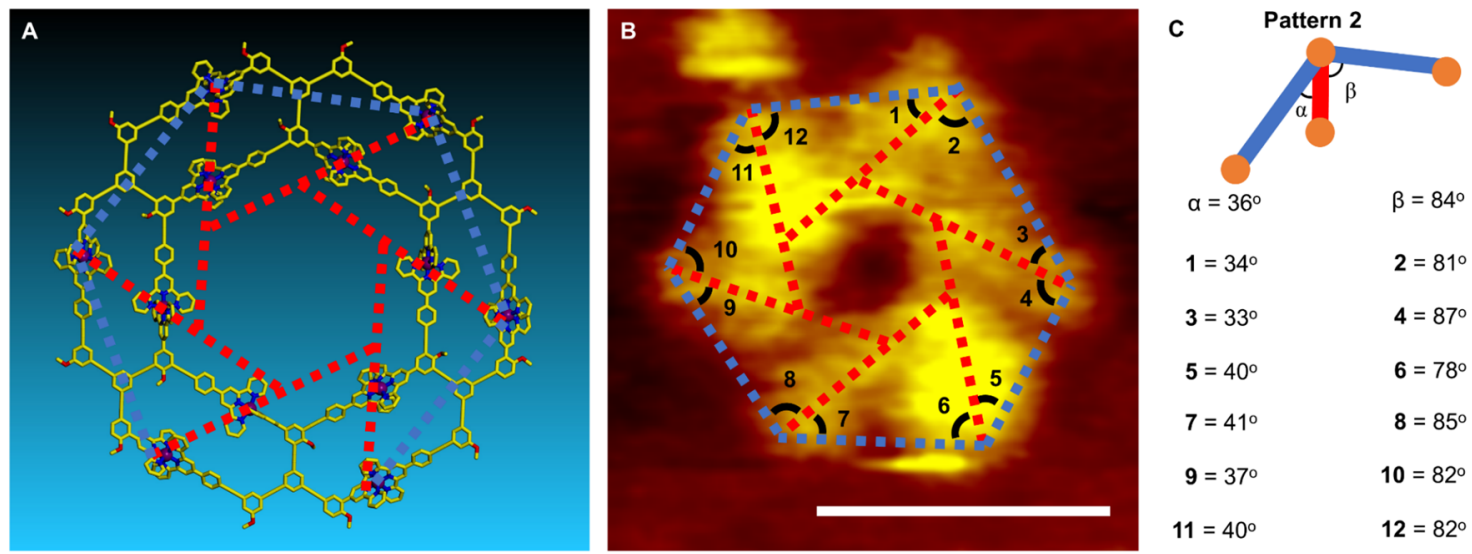

Figure S156. The comparison of (A) modeling structure and (B) STM image of isomer A2 with pattern labeled and measured. (C) Angles of the pattern measured in STM image.
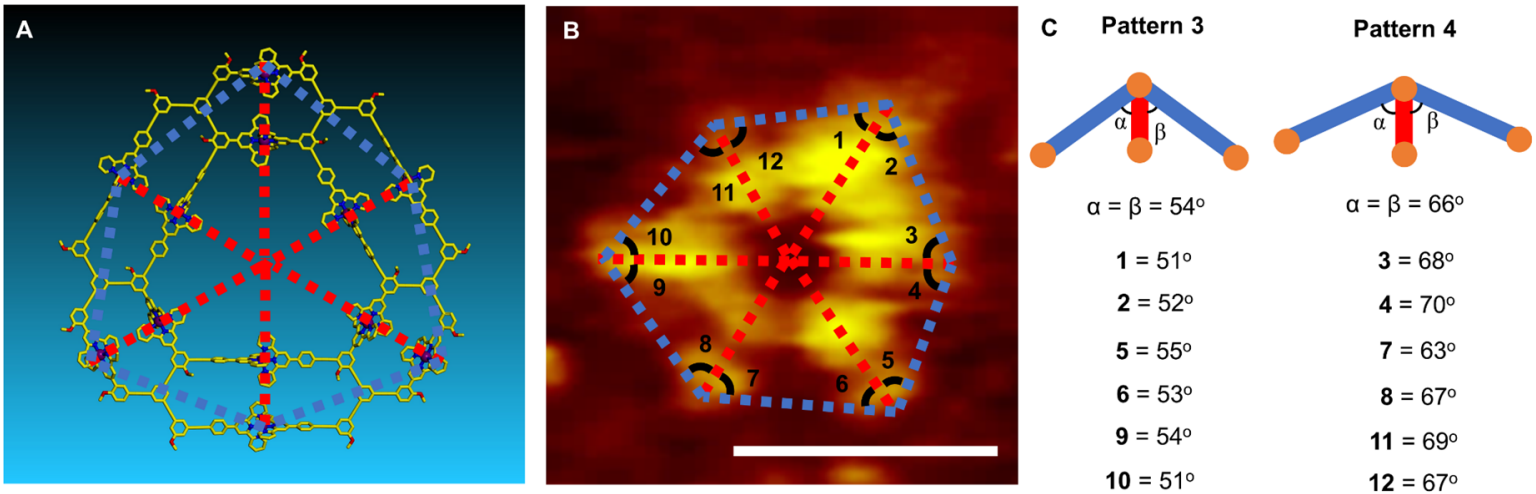

Figure S157. The comparison of (A) modeling structure and (B) STM image of isomer A3 with pattern labeled and measured. (C) Angles of the pattern measured in STM image.
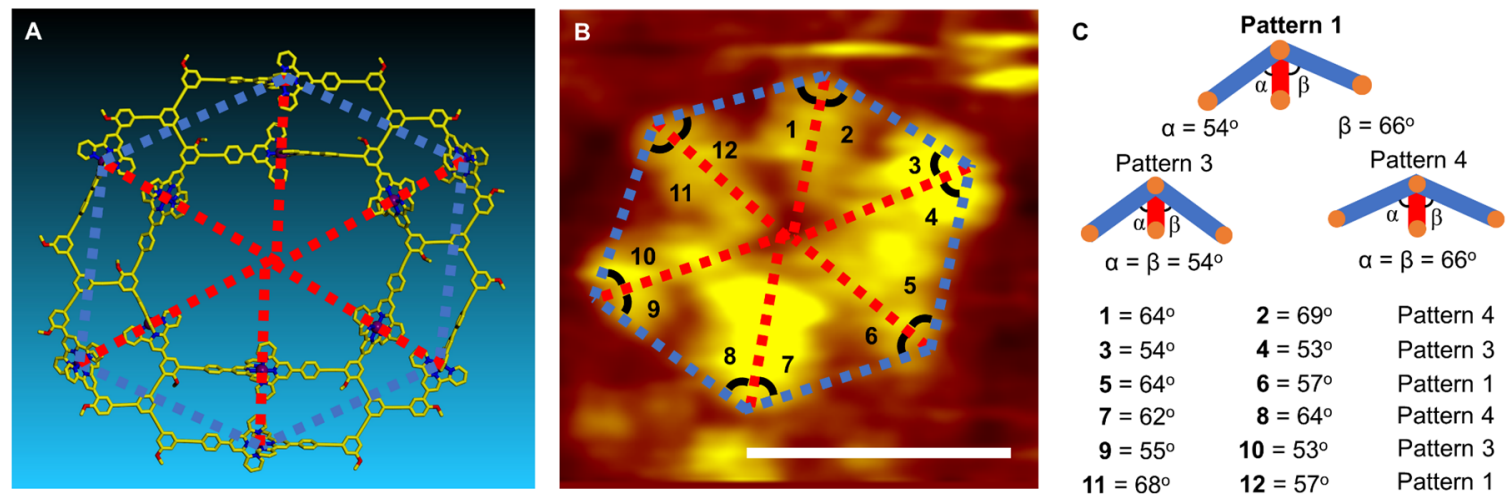

Figure S158. The comparison of (A) modeling structure and (B) STM image of isomer A4 with pattern labeled and measured. (C) Angles of the pattern measured in STM image. 

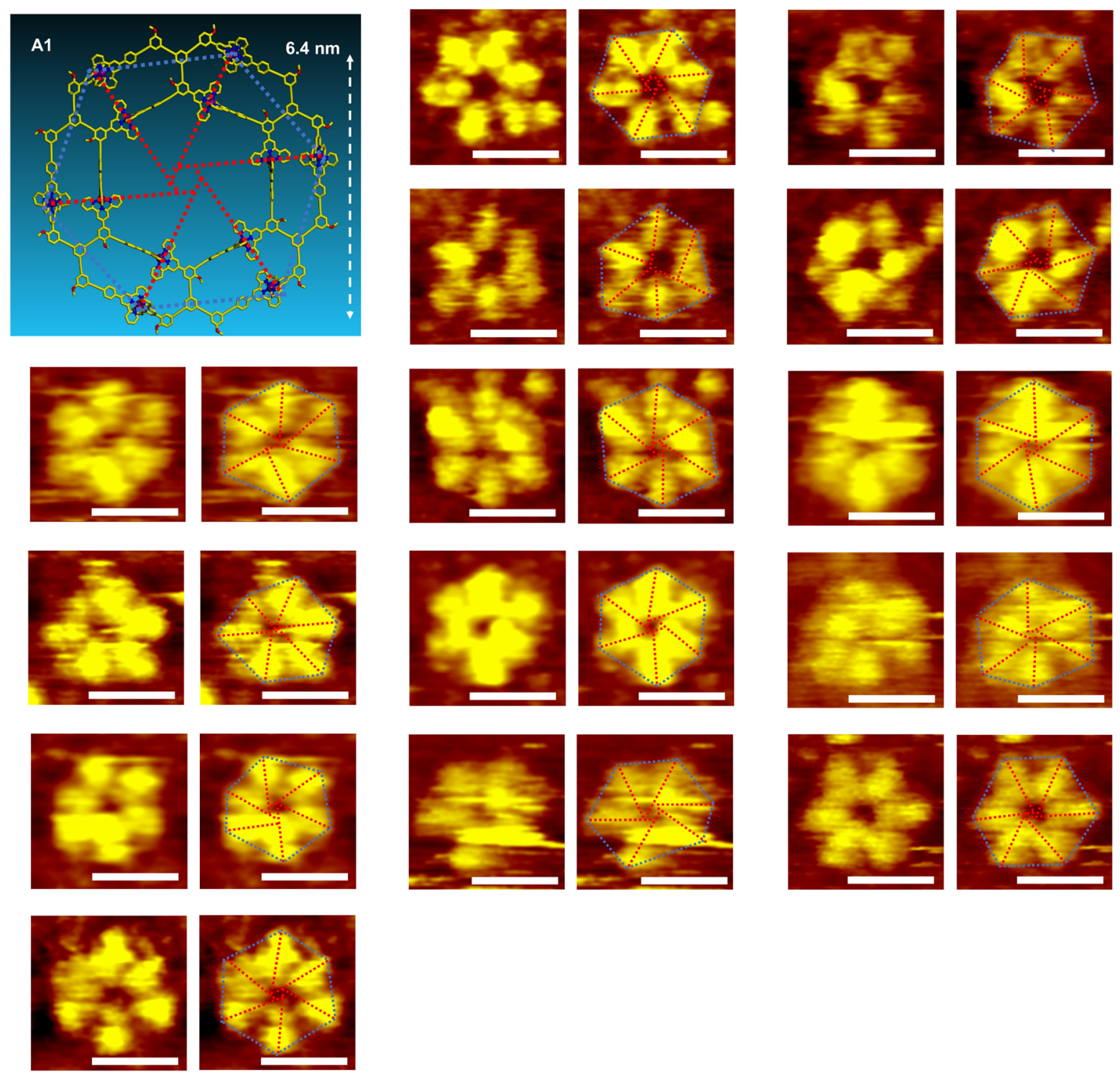

Figure S159. STM images of isomer A1 with similar pattern labeled, scale bar of $5 \mathrm{~nm}$. 

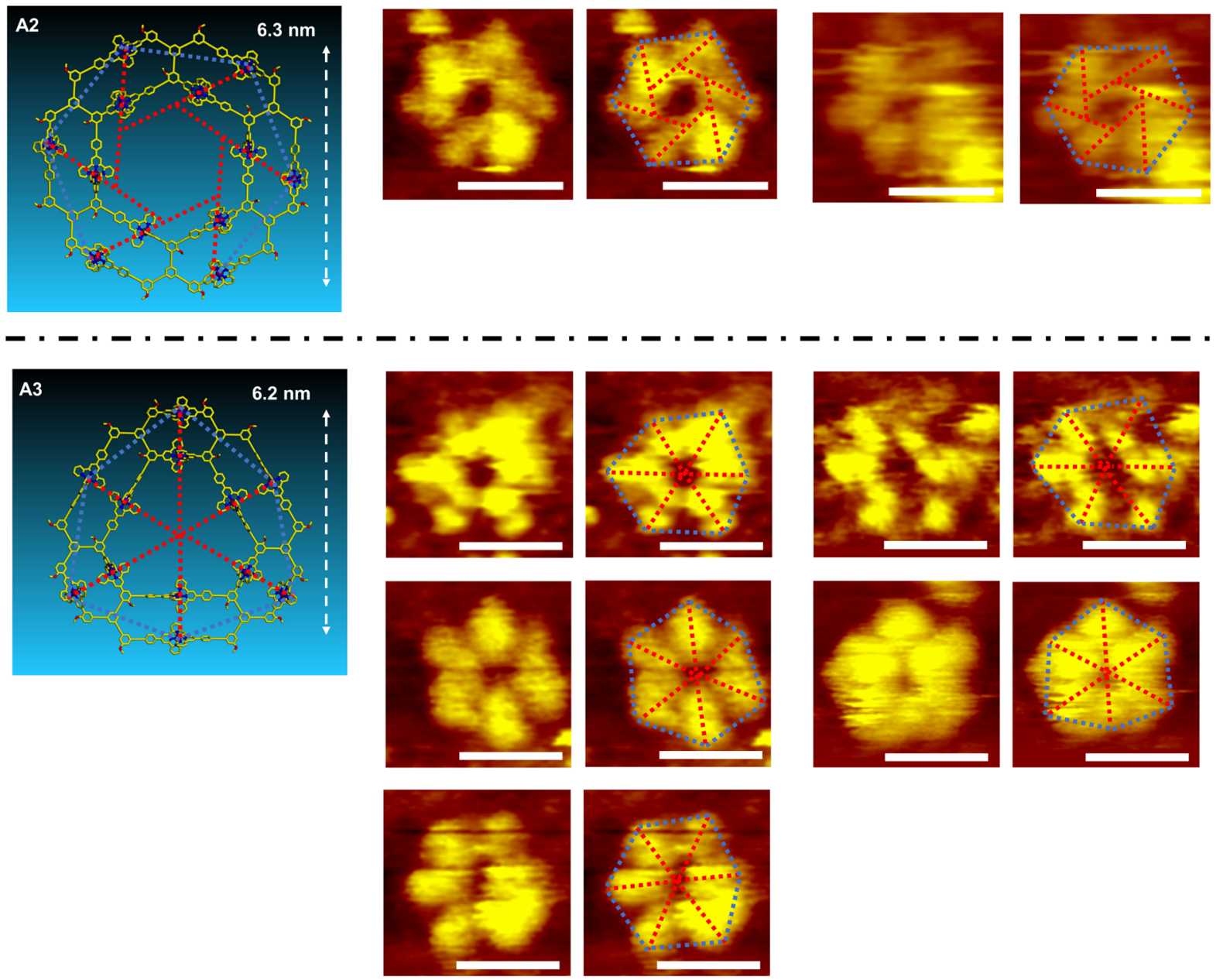

Figure S160. STM images of isomers A2 and A3 with corresponding patterns labeled, scale bar of $5 \mathrm{~nm}$. 

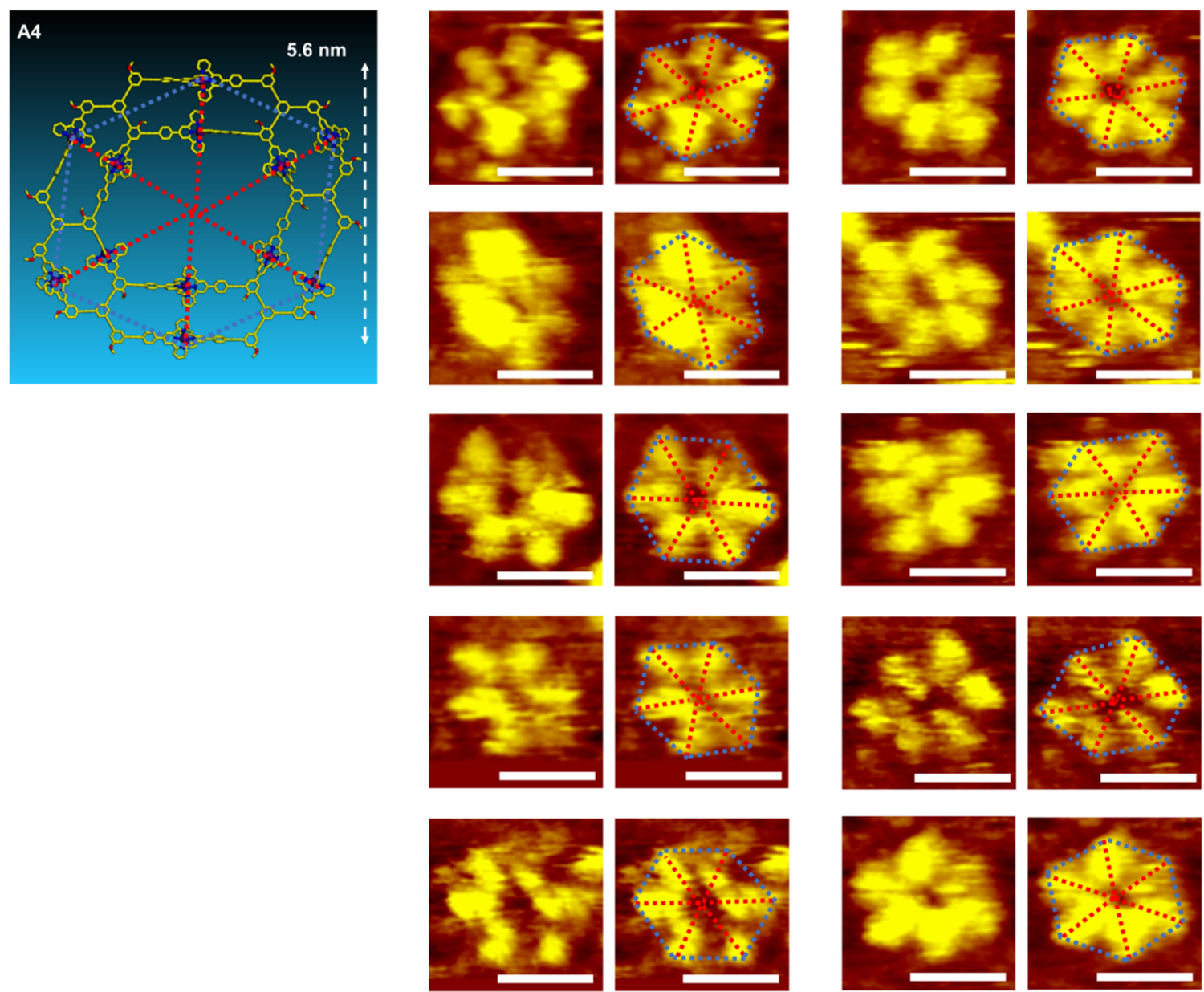

Figure S161. STM images of isomer A4 with specific pattern labeled, scale bar of $5 \mathrm{~nm}$.
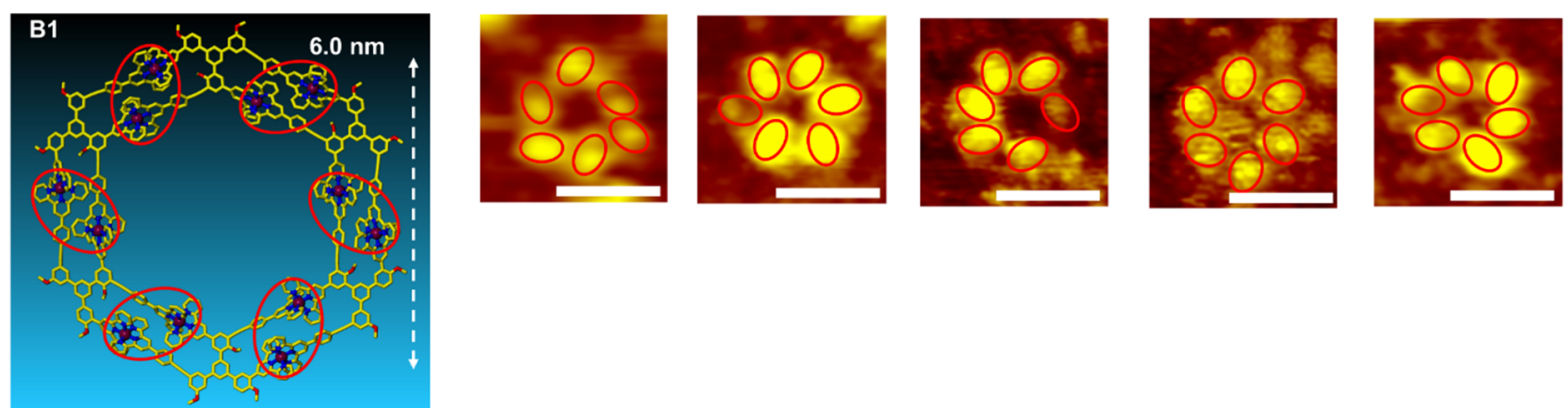

Figure S162. STM image of supramolecule B1 at the scale bar of $5 \mathrm{~nm}$. 

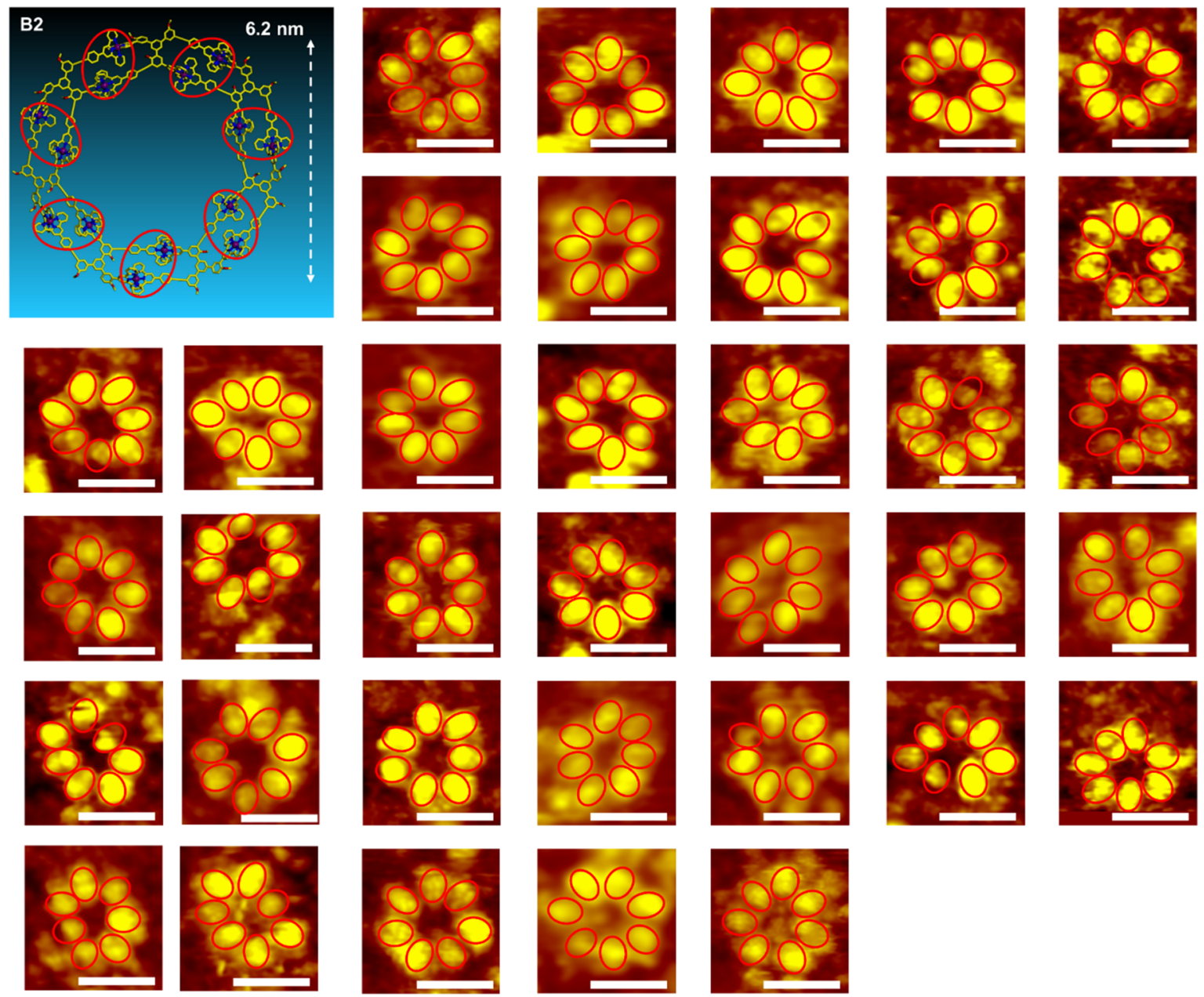

Figure S163. STM image of supramolecule B2 at the scale bar of $5 \mathrm{~nm}$.
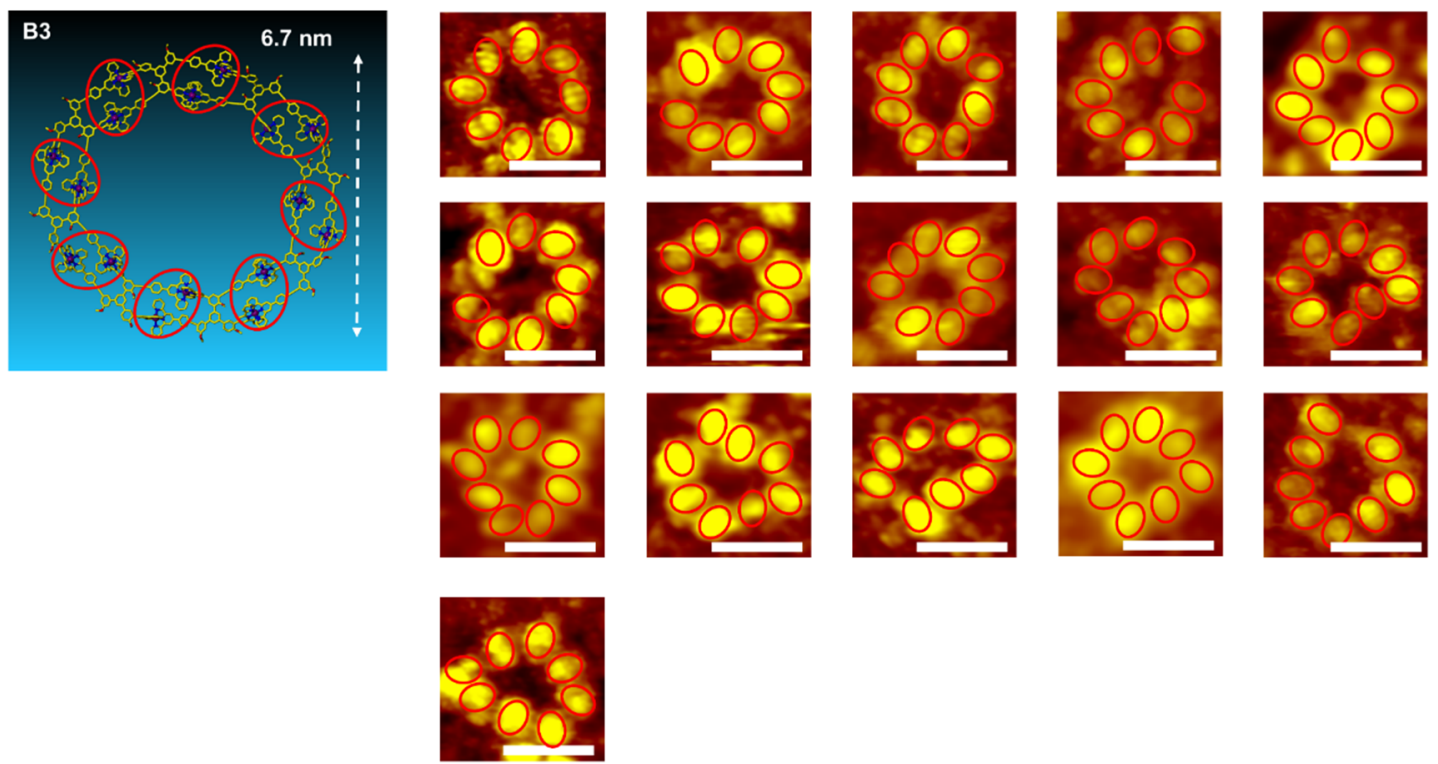

Figure S164. STM image of supramolecule B3 at the scale bar of $5 \mathrm{~nm}$. 


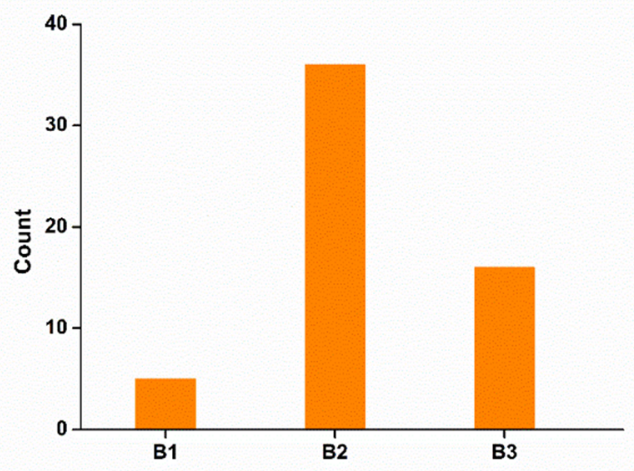

Figure S165. Distribution of three supramolecules based on STM image for B1 $\left(\mathbf{Z n}_{12} \mathbf{L B}_{\mathbf{6}}\right)$, B2 $\left(\mathbf{Z n}_{14} \mathbf{L B}_{7}\right)$ and $\mathbf{B 3}\left(\mathbf{Z n}_{16} \mathbf{L} \mathbf{B}_{\mathbf{8}}\right)$. 


\section{Reference}

1. Wang, M.; Wang, C.; Hao, X.-Q.; Liu, J.; Li, X.; Xu, C.; Lopez, A.; Sun, L.; Song, M.-P.; Yang, H. B.; Li, X. Hexagon Wreaths: Self-Assembly of Discrete Supramolecular Fractal Architectures Using Multitopic Terpyridine Ligands. J. Am. Chem. Soc. 2014, 136 (18), 6664-6671.

2. Wang, L.; Liu, R.; Gu, J.; Song, B.; Wang, H.; Jiang, X.; Zhang, K.; Han, X.; Hao, X.-Q.; Bai, S.; Wang, M.; Li, X.; Xu, B.; Li, X. Self-Assembly of Supramolecular Fractals from Generation 1 to 5. J. Am. Chem. Soc. 2018, 140 (43), 14087-14096.

3. Zhang, Z.; Wang, H.; Wang, X.; Li, Y.; Song, B.; Bolarinwa, O.; Reese, R. A.; Zhang, T.; Wang, X.-Q.; Cai, J.; Xu, B.; Wang, M.; Liu, C.; Yang, H.-B.; Li, X. Supersnowflakes: Stepwise Self-Assembly and Dynamic Exchange of Rhombus Star-Shaped Supramolecules. J. Am. Chem. Soc. 2017, 139 (24), 8174-8185.

4. Yin, G.-Q.; Wang, H.; Wang, X.-Q.; Song, B.; Chen, L.-J.; Wang, L.; Hao, X.-Q.; Yang, H.-B.; Li, $\mathrm{X}$. Self-Assembly of Emissive Supramolecular Rosettes with Increasing Complexity Using Multitopic Terpyridine Ligands. Nat. Commun. 2018, 9 (1), 567. 